Prepared in cooperation with the State of Wisconsin and local agencies

\title{
Water-Quality and Lake-Stage Data for Wisconsin Lakes, Water Year 2014
}

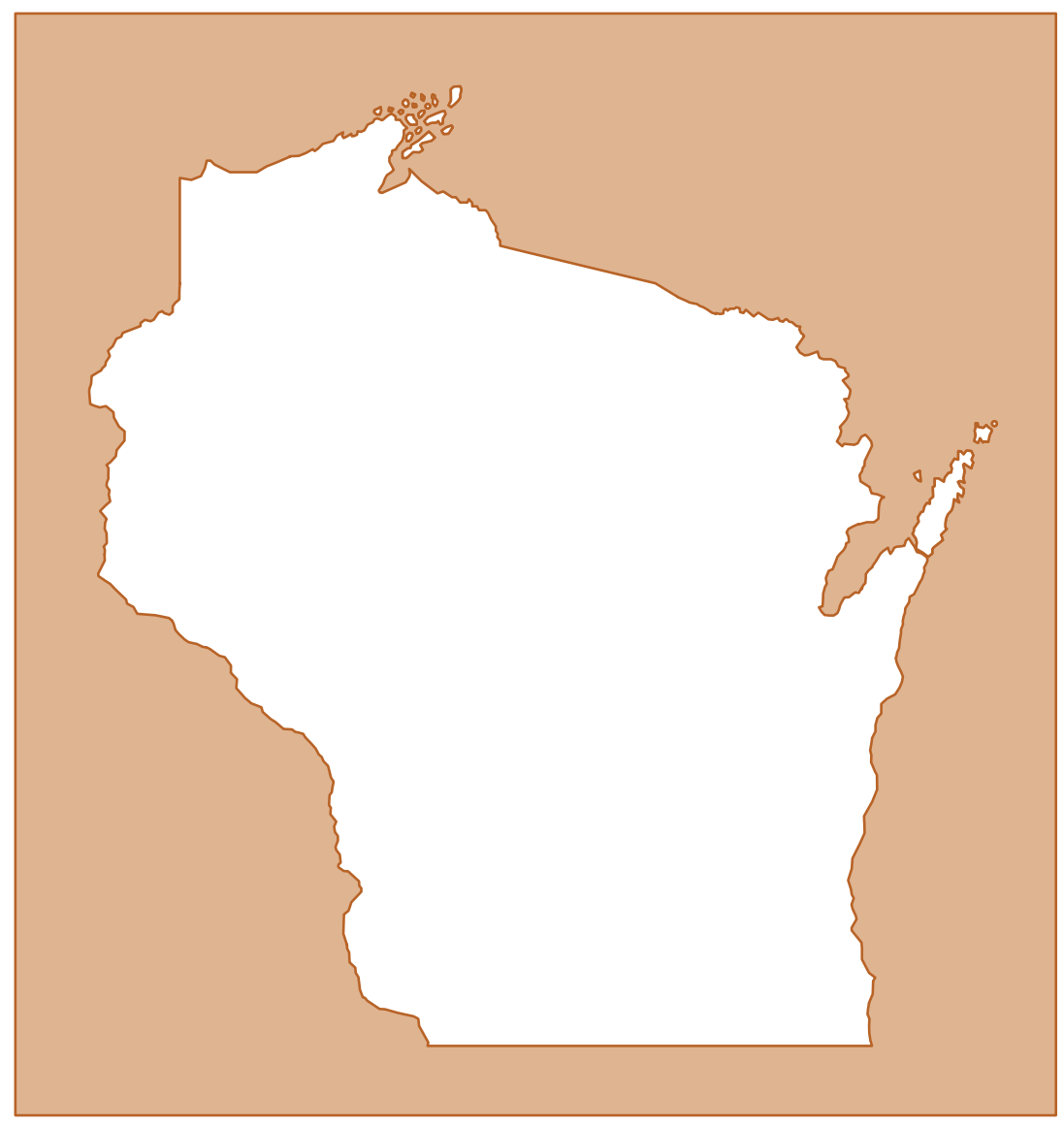

Open-File Report 2016-1131 



\section{Water-Quality and Lake-Stage Data for Wisconsin Lakes, Water Year 2014}

By S. Bridgett Manteufel and Dale M. Robertson

Prepared in cooperation with the State of Wisconsin and with other agencies

Open-File Report 2016-1131 


\title{
U.S. Department of the Interior SALLY JEWELL, Secretary
}

\section{U.S. Geological Survey Suzette Kimball, Acting Director}

\author{
U.S. Geological Survey, Reston, Virginia: 2016
}

For more information on the USGS - the Federal source for science about the Earth, its natural and living resources, natural hazards, and the environment-visit http://www.usgs.gov or call 1-888-ASK-USGS.

For an overview of USGS information products, including maps, imagery, and publications, visit http://store.usgs.gov.

Any use of trade, firm, or product names is for descriptive purposes only and does not imply endorsement by the U.S. Government.

Although this information product, for the most part, is in the public domain, it also may contain copyrighted materials as noted in the text. Permission to reproduce copyrighted items must be secured from the copyright owner.

Suggested citation:

Manteufel, S.B., and Robertson, D.M., 2017, Water-quality and lake-stage data for Wisconsin lakes, water year 2014: U.S. Geological Survey Open-File Report 2016-1131, 170 p.,

https://doi.org/10.3133/ofr20161131.

ISSN 2328-0328 (online) 


\section{Contents}

Introduction

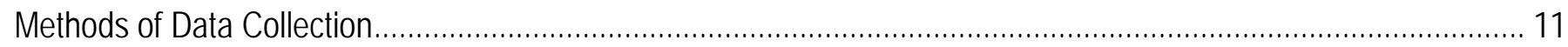

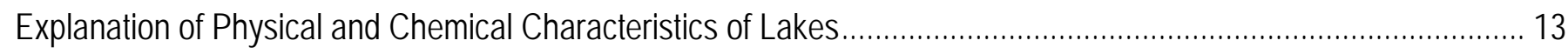

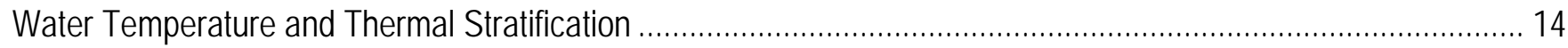

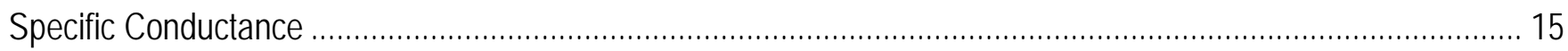

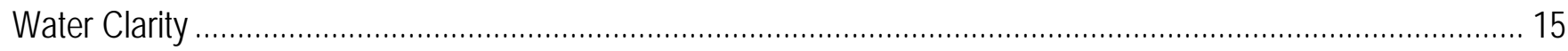

$\mathrm{pH}$

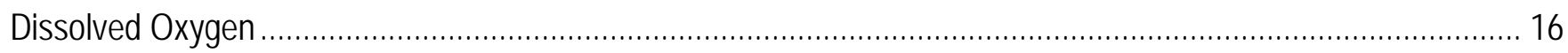

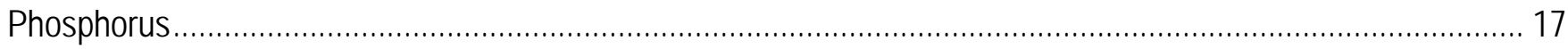

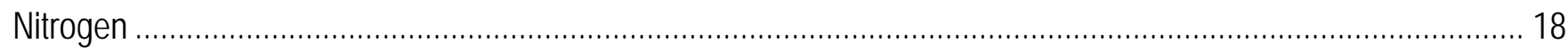

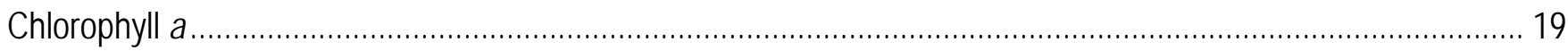

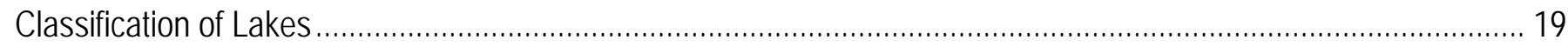

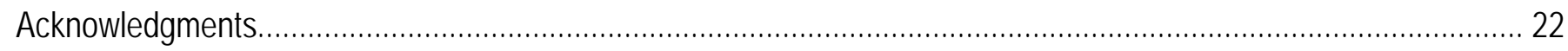

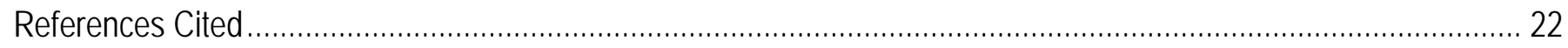

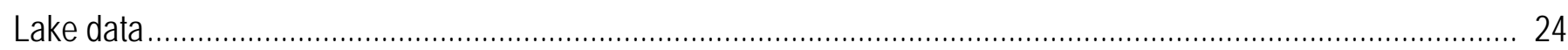

Anvil

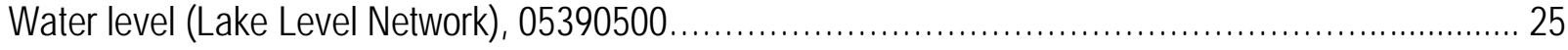

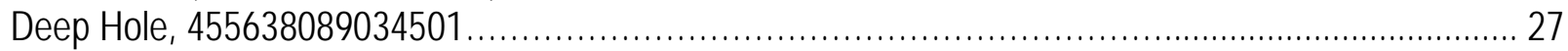

Beulah

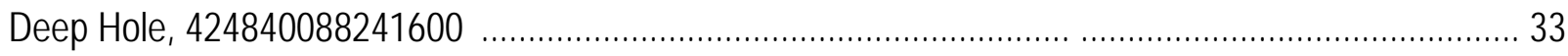

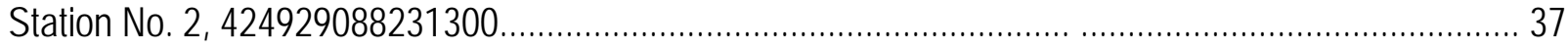

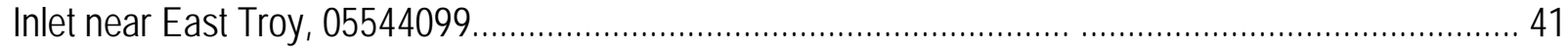

Big Cedar

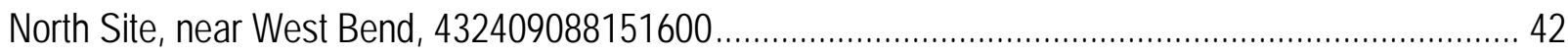

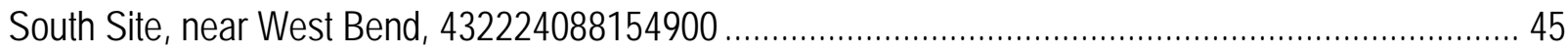

Delavan

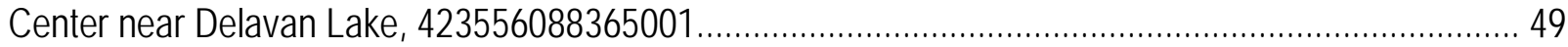

North End near Lake Lawn, 423659088354401................................................................... 56

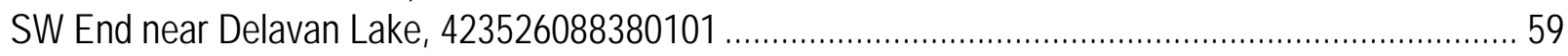

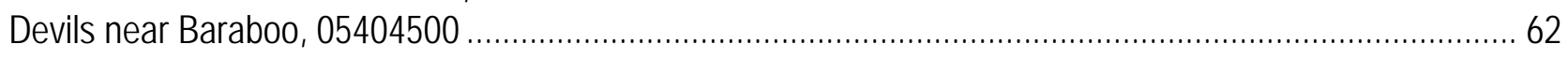

Geneva

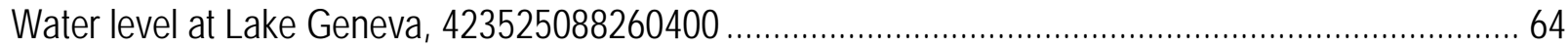

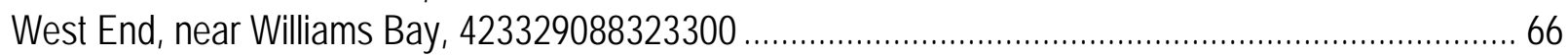


Green

Water level at Green Lake, 434840089000001

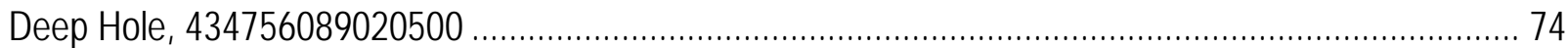

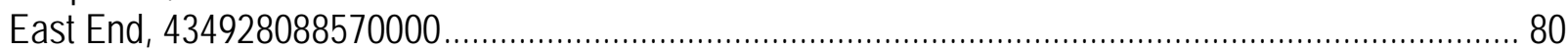

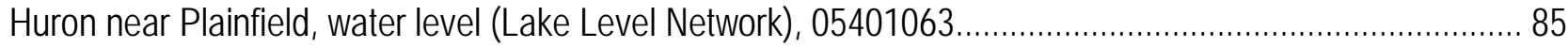

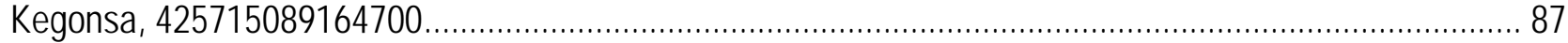

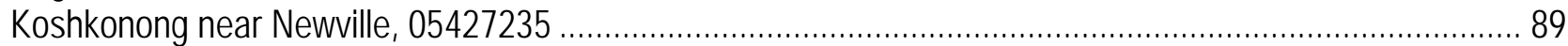

Long Lake near Saxeville, water level (Lake Level Network), 441257089071500 ..................................... 91

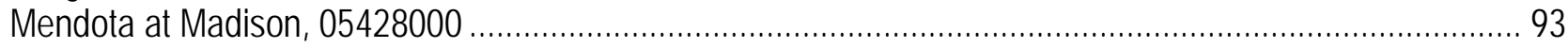

Middle Genesee at Genesee Lake Road, near Oconomowoc, 430251088284700 ................................. 95

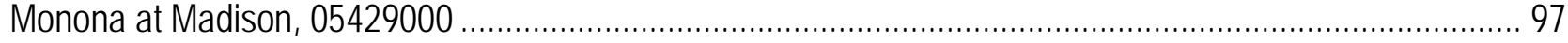

North Lake near Elkhorn, water level (Lake Level Network), 05545000 ............................................... 100

Oconomowoc

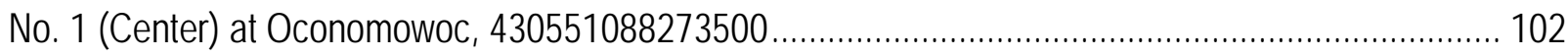

No. 2 (off Hewitt Point) at Oconomowoc, 430609088262200........................................................ 106

Okauchee

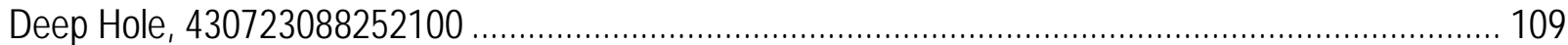

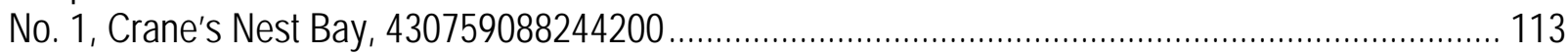

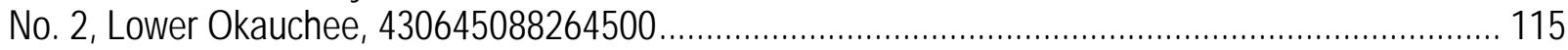

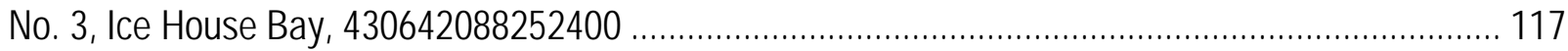

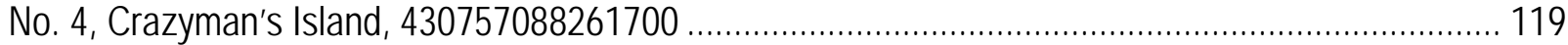

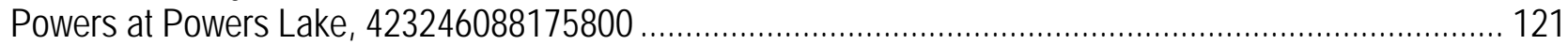

Silver Lake near Cumberland, water level (Lake Level Network), 453420091551600 _............................. 125

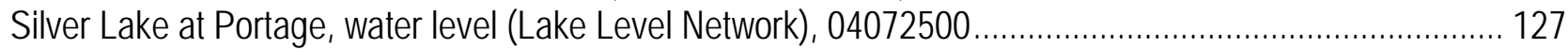

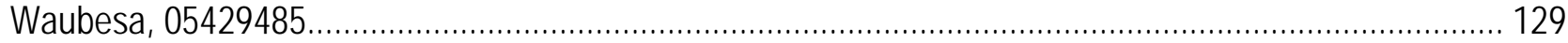

Whitefish (Bardon) Lake near Gordon, water level (Lake Level Network), 461231091524900.................. 132

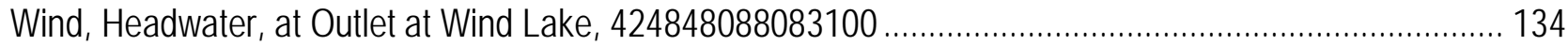

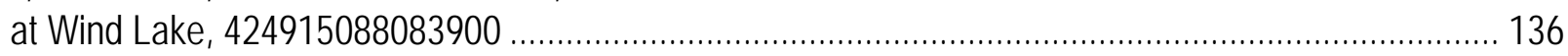

Winnebago

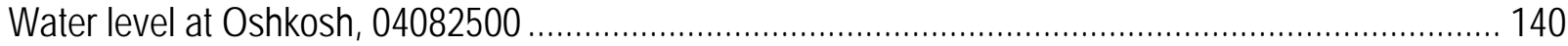

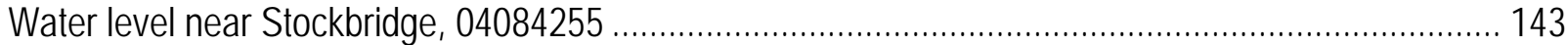

Wisconsin Water Science Center publications pertaining to lakes............................................................. 145

Appendix - Wisconsin Lakes Team Quality-Assurance Plan...................................................................... 156

\section{Figures}

1. Map showing the locations of U.S. Geological Survey water-quality, lake-stage, and lake-level network stations in

Wisconsin.

\section{Tables}

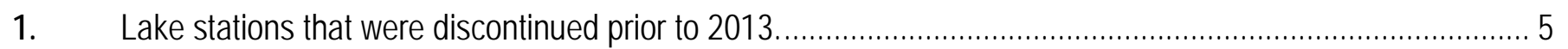

2. Parameter identification numbers and laboratory reporting levels for chemical parameters commonly measured in lakes, and analyzed at the National Water Quality Laboratory or the Wisconsin State Laboratory of Hygiene.12 


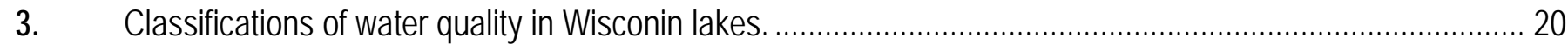

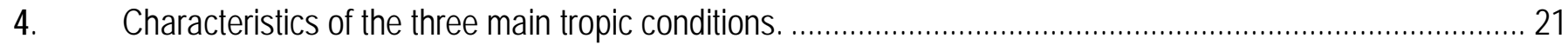




\section{Conversion Factors}

Inch/Pound to International System of Units

\begin{tabular}{|c|c|c|}
\hline Multiply & By & To obtain \\
\hline \multicolumn{3}{|c|}{ Length } \\
\hline foot $(\mathrm{ft})$ & 0.3048 & meter (m) \\
\hline mile (mi) & 1.609 & kilometer (km) \\
\hline yard (yd) & 0.9144 & meter $(\mathrm{m})$ \\
\hline \multicolumn{3}{|c|}{ Area } \\
\hline acre & 0.4047 & hectare (ha) \\
\hline square mile $\left(\mathrm{mi}^{2}\right)$ & 259.0 & hectare (ha) \\
\hline square mile $\left(\mathrm{mi}^{2}\right)$ & 2.590 & square kilometer $\left(\mathrm{km}^{2}\right)$ \\
\hline \multicolumn{3}{|c|}{ Volume } \\
\hline gallon (gal) & 3.785 & liter (L) \\
\hline \multicolumn{3}{|c|}{ International System of Units to Inch/Pound } \\
\hline Multiply & By & To obtain \\
\hline \multicolumn{3}{|c|}{ Length } \\
\hline centimeter $(\mathrm{cm})$ & 0.3937 & inch (in.) \\
\hline meter (m) & 3.281 & foot $(\mathrm{ft})$ \\
\hline meter (m) & 1.094 & yard (yd) \\
\hline centimeter $(\mathrm{cm})$ & 0.0001 & micrometer $(\mu \mathrm{m})$ \\
\hline \multicolumn{3}{|c|}{ Volume } \\
\hline liter (L) & 0.2642 & gallon (gal) \\
\hline
\end{tabular}

Temperature in degrees Celsius $\left({ }^{\circ} \mathrm{C}\right)$ may be converted to degrees Fahrenheit $\left({ }^{\circ} \mathrm{F}\right)$ as ${ }^{\circ} \mathrm{F}=\left(1.8 \times{ }^{\circ} \mathrm{C}\right)+32$.

\section{Datum}

Sea level: In this report "sea level" refers to either the National Geodetic Vertical Datum of 1929 (NGVD of 1929)—a geodetic datum derived from a general adjustment of the first-order level nets of both the United States and Canada, formerly called Sea Level Datum of 1929—or the North American Vertical Datum of 1988 (NAVD 88).

\section{Supplemental Information}

Specific conductance of water is expressed in microsiemens per centimeter at 25 degrees Celsius $\left(\mu \mathrm{S} / \mathrm{cm}\right.$ at $\left.25^{\circ} \mathrm{C}\right)$. This unit is equivalent to micromhos per centimeter $\mu \mathrm{mho} / \mathrm{cm}$ at $25^{\circ} \mathrm{C}$, formerly used by the U.S. Geological Survey. 
Abbreviated water-quality units: Chemical concentrations and water temperature are given in metric units. Chemical concentration is given in

milligrams per liter (mg/L) or micrograms per liter $(\mu \mathrm{g} / \mathrm{L})$. Milligram per liter is a unit expressing the concentration of chemical constituents in solution as weight (milligrams) of solute per unit volume (liter) of water. One thousand micrograms per liter is equivalent to one milligram per liter. For water with dissolved-solids concentrations less than 7,000 $\mathrm{mg} / \mathrm{L}$, the numerical values for concentrations expressed as $\mathrm{mg} / \mathrm{L}$ and $\mu \mathrm{g} / \mathrm{L}$ are the same as for concentrations in parts per million and parts per billion, respectively. 


\section{Water-quality and Lake-stage Data for Wisconsin Lakes, Water}

\section{Year 2014}

By Wisconsin Water Science Center Lake-Studies Team

\section{Introduction}

The U.S. Geological Survey (USGS), in cooperation with local and other agencies, collects data at selected lakes throughout Wisconsin. These data, accumulated over many years, provide a database for developing an improved understanding of the water quality of lakes. To make these data available to interested parties outside the USGS, the data are published annually in this report series. The locations of water-quality and lake-stage stations in Wisconsin for water year 2014 are shown in figure 1. A water year is the 12-month period from October 1 through September 30. It is designated by the calendar year in which it ends. Thus, the period October 1, 2013, through September 30, 2014, is called “water year 2014.”

The purpose of this report is to provide information about the chemical and physical characteristics of Wisconsin lakes. Data that have been collected at specific lakes, and information to aid in the interpretation of those data, are included in this report. Data collected include measurements of in-lake water quality and lake stage. Time series of Secchi depths, surface total phosphorus, and chlorophyll $a$ concentrations collected during nonfrozen periods are included for many lakes. Graphs of vertical profiles of temperature, dissolved oxygen, $\mathrm{pH}$, and specific conductance are included for sites where these parameters were measured. Descriptive information for each lake includes the location of the lake, area of the lake's watershed, period for which data are available, revisions to previously published records, and pertinent remarks. Additional data, such as streamflow 
and water quality in tributary and outlet streams of some of the lakes, are published online at http://nwis.waterdata.usgs.gov/wi/nwis.

Water-resources data, including stage and discharge data at most streamflow-gaging stations, are available through the World Wide Web on the Internet. The Wisconsin Water Science Center's home page is at http://wi.water.usgs.gov/. Information about the Wisconsin Water Science Center's Lakes Program is found at http://wi.water.usgs.gov/lakes/index.html and http://wi.water.usgs.gov/projects/index.html. The Wisconsin Water Science Center Lake-Studies Team includes S.B. Manteufel, D.M. Robertson, B.J. Siebers, and E.D. Dantoin. Figure 1. Map showing the locations of U.S. Geological Survey water-quality, lake-stage, and lake-level network stations in Wisconsin. 


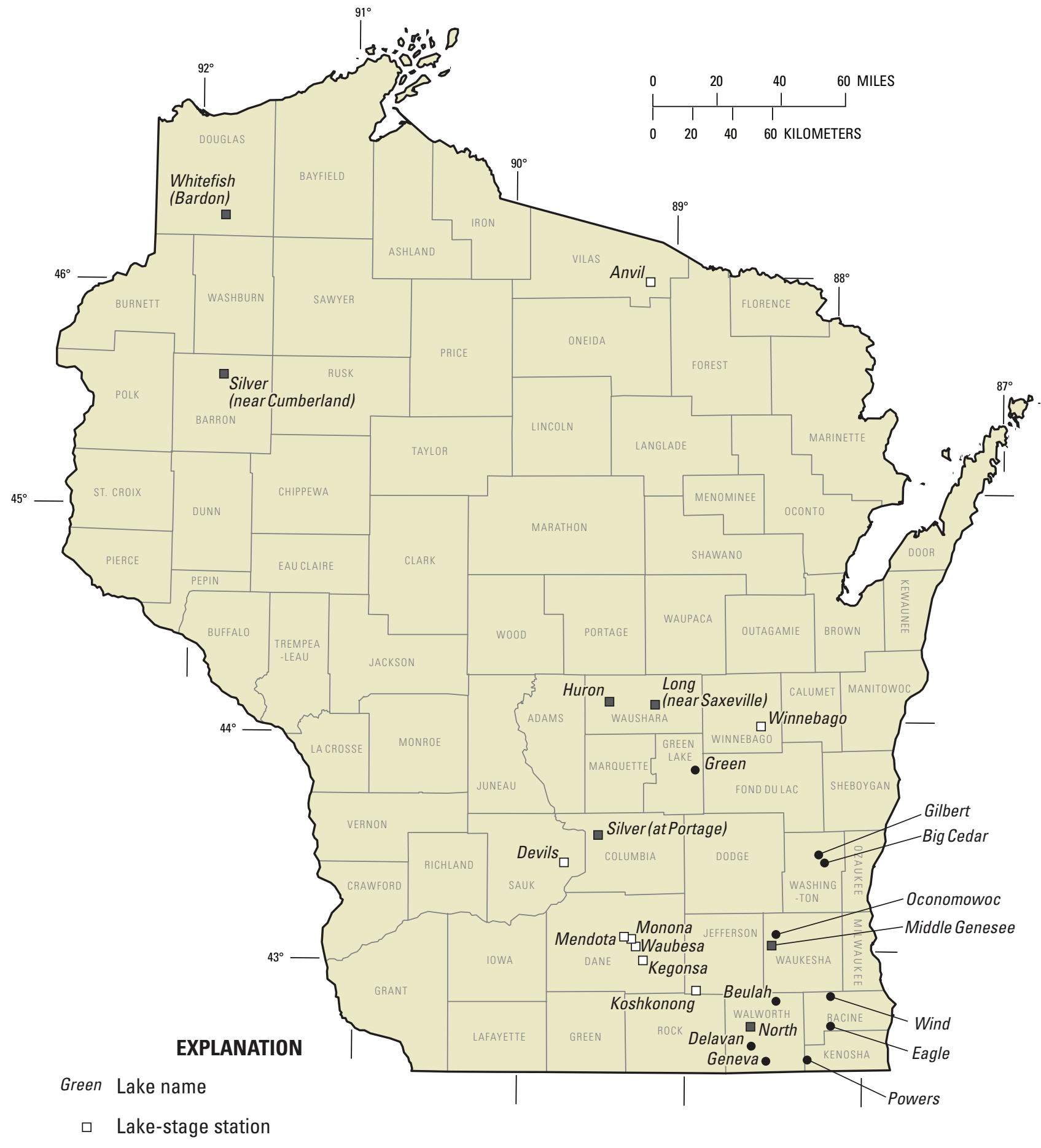

- Lake-level network station

- Lake water-quality and stage station

Note: at some lakes more than one site may be monitored.

Figure 1. Location of USGS lake water-quality, lake-stage, and lake-level network stations in Wisconsin. 
The USGS has monitored lakes in cooperation with local and other agencies since 1983.

Cooperators in 2014 included:

Big Cedar Lake Protection and Rehabilitation District

Dane County

Delavan Lake Sanitary District

Geneva Lake Environmental Agency

Green Lake Sanitary District

Lake Beulah Management District

Middle Genesee Lake District

Okauchee Lake Management District

Powers Lake District

Rock County Public Works Department

Town of Delavan

Town of Washington

U.S. Army Corps of Engineers

U.S. Environmental Protection Agency

Village of Oconomowoc Lake

Wind Lake Management District

Wisconsin Department of Natural Resources 
Lake data-collection sites are identified by a unique identification number. Lake waterquality sites are identified by a 15-digit number that is a concatenation of the site's latitude, longitude, and a two-digit sequence number. The sequence number is used to distinguish between sites located at the same latitude-longitude designation. The site identification number is permanently assigned to the site; the actual latitude and longitude of the site are subject to revision and are stored separately. For some lakes, which have historical records of lake stage, an 8-10 digit number is assigned according to downstream order. Gaps are left in the numerical series to allow for new stations; hence, the numbers are not consecutive. The first two digits of the complete 8-10 digit number, such as 04087000 or 054310157 , designate the major river basin. For example, “04” designates the St. Lawrence River Basin, and “05” designates the Upper Mississippi River Basin. The water-quality lake stations that were discontinued prior to water year 2013 are listed in table 1. Discontinued lake-stage stations are not included in this table.

Table 1. Lake monitoring stations that were discontinued prior to 2013._Continued [NW, northwest; SE, southeast; SW, southwest; QW, water-quality station; (a), Wisconsin Valley Improvement Co. currently collects stage data for this site]

\begin{tabular}{|c|c|c|}
\hline Alma Lake, near St. Germain & 455426089254700 & $\begin{array}{l}\text { Oct. 1984-Sept. 1990, } \\
\text { May 1992-Sept. } 1996\end{array}$ \\
\hline Balsam Lake, off Cedar Island, at Balsam Lake & 452755092264600 & Feb. 1991-Aug. 1994 \\
\hline off Little Narrows, near Balsam Lake & 452858092265300 & May 1991-Aug. 1994 \\
\hline Balsam Lake, near Birchwood & 453907091345800 & $\begin{array}{l}\text { Mar. 1993-Aug. 1994, } \\
\text { Mar. 1996-Aug. 1997, } \\
\text { Mar.-Sept. } 2001\end{array}$ \\
\hline Bass Lake, near Shawano & 445215088300300 & Feb. 1990-Aug. 1992 \\
\hline Bear Lake at Deep Hole near Haugen & 453754091490900 & Mar. 1992-Aug. 1993 \\
\hline
\end{tabular}


Table 1. Lake monitoring stations that were discontinued prior to 2013.—Continued

[NW, northwest; SE, southeast; SW, southwest; QW, water-quality station; (a), Wisconsin Valley Improvement Co. currently collects stage data for this site]

\begin{tabular}{|c|c|c|}
\hline Station name & Site identification number & Period of record \\
\hline North end, near Beaver Dam & 433122088545700 & June-Oct. 1991 \\
\hline Benedict Lake, near Powers Lake & 423201088180800 & May 1998-Aug. 2000 \\
\hline Big Blacksmith Lake, near Keshena & 445401088334500 & Feb. 1990-Aug. 1992 \\
\hline Big Hills (Hills) Lake, near Wild Rose & 440912089092000 & $\begin{array}{l}\text { June 1983-Aug. 1984, } \\
\text { Feb.-Aug. 1987, } \\
\text { Feb.-Aug. 1990, } \\
\text { Feb.-Aug. 1993, } \\
\text { Feb.-Aug. 1996, } \\
\text { Feb.-Aug. 1999 }\end{array}$ \\
\hline Big Muskego Lake, at North Site, near Muskego & 425301088061300 & Feb.-Aug. 1988 \\
\hline Research Base, near Muskego & 425235088075300 & May-June 1994 \\
\hline Big Round Lake, near Milltown & 453142092180100 & Feb.-Sept. 2001 \\
\hline Big St. Germain Lake, near St. Germain & 455557089311000 & Feb. 1992-Aug. 1996 \\
\hline near Lake Tomahawk & 05390750 & 1991-2001 \\
\hline Big Sand Lake, Deep Hole, near Hertel & 454910092134000 & Feb.-Sept. 2001 \\
\hline East Site, near Hertel & 454921092124300 & Feb.-Sept. 2001 \\
\hline Big Sissabagama Lake, near Stone Lake & 454724091303600 & $\begin{array}{l}\text { Apr. 1986-Sept. 1996, } \\
\text { Oct. 1997-Sept. } 2002\end{array}$ \\
\hline North Site, near Stone Lake & 454800091312900 & Mar. 1998-Sept. 2001 \\
\hline Booth Lake, near East Troy & 424800088254800 & $\begin{array}{l}\text { Feb. 1992-Aug. 1994, } \\
\text { Feb. 2001-Aug. } 2003\end{array}$ \\
\hline Buffalo Lake, Center Site, at Packwaukee & 434558089260600 & May 1998-Sept. 2001 \\
\hline East End, at Montello & 434720089201600 & May 1998-Sept. 2001 \\
\hline West End, near Endeavor & 434414089282400 & May 1998-Sept. 2001 \\
\hline Butternut Lake, near Park Falls & 455854090310300 & Oct. 2002-Oct. 2004 \\
\hline Deep Hole, near Park Falls & 455803090310800 & Mar. 2003-Sept. 2004 \\
\hline North Site, near Butternut & 455904090303400 & Mar. 2003-Sept. 2004 \\
\hline Far South Site, near Park Falls & 455651090312700 & Mar. 2003-Sept. 2004 \\
\hline Denoon Lake, at Wind Lake & 425044088100300 & Feb. 1991-Aug. 1996 \\
\hline Druid Lake near Hartford & 431643088243300 & Feb. 1991-Sept. 1996 \\
\hline Eagle Lake near Kansasville & 05544500 & $\begin{array}{l}\text { 1936-64, 1975-77, 1979, } \\
\text { Feb. 1993-Sept. } 1996\end{array}$ \\
\hline Eagle Lake, at Deep Hole, near Kansasville & 424207088072400 & Feb. 1993-Aug. 1996 \\
\hline Eagle Spring Lake, at Eagleville & 425103088261500 & Apr. 1991-Sept. 2001 \\
\hline Elizabeth Lake, near Twin Lakes & 423051088155300 & Feb. 1995-Sept. 1997 \\
\hline Fish Lake, near Sauk City & 05406050 & Nov. 1966-Sept. 1981, \\
\hline
\end{tabular}


Table 1. Lake monitoring stations that were discontinued prior to 2013.—Continued

[NW, northwest; SE, southeast; SW, southwest; QW, water-quality station; (a), Wisconsin Valley Improvement Co. currently collects stage data for this site]

\begin{tabular}{|c|c|c|}
\hline Station name & Site identification number & Period of record \\
\hline & & $\begin{array}{l}\text { Apr. 1985-May 1987, } \\
\text { May 1988, Apr. 1989-Oct. 1990, } \\
\text { Oct. 1990-Nov. 1996, } \\
\text { Nov. 1996-Sept. 2004 }\end{array}$ \\
\hline Fowler Lake, Center, at Oconomowoc & 430653088294601 & $\begin{array}{l}\text { Jan.-Dec. 1984, } \\
\text { Oct. 1986-Sept. } 1996\end{array}$ \\
\hline Fox Lake, Deep Hole, at Fox Lake & 433458088560600 & June 1991-Mar. 1993 \\
\hline Gilbert Lake Deep Hole, near West Bend & 432504088152201 & Apr. -July 2012 \\
\hline Gilbert Lake Tributary, near West Bend & 04086418 & Apr. -July 2012 \\
\hline Gilbert Lake Spring \#1, near West Bend & 432514088151601 & Apr. -July 2012 \\
\hline Gilbert Lake Spring \#2, near West Bend & 432511088151801 & Apr. -July 2012 \\
\hline Geneva Lake, Geneva Bay, at Lake Geneva & 423455088263800 & Apr. 1997-Feb. 1999 \\
\hline Williams Bay, at Williams Bay & 423420088320500 & Apr. 1997-Feb. 1999 \\
\hline East End, near Lake Geneva & 423421088272300 & Apr. 1997-May 2000 \\
\hline Hemlock Lake, near Mikana & 453421091333700 & $\begin{array}{l}\text { Mar. 1993-Aug. 1994, } \\
\text { Mar. 1996-Aug. 1997, } \\
\text { Mar.-Sept. } 2001\end{array}$ \\
\hline Hooker Lake, at Salem & 423335088060300 & Feb. 1992-Aug. 1993 \\
\hline Kawaguesaga, Deep Hole, near Minocqua & 455208089435800 & May-Sept. 2003 \\
\hline South Site, near Minocqua & 455145089442600 & May-Sept. 2003 \\
\hline Kirby Lake, near Cumberland & 453554092042101 & Nov. 1995-Oct. 1996 \\
\hline (Site 1) near Cumberland & 453608092035801 & Nov. 1995-Nov. 1996 \\
\hline (Site 2) near Cumberland & 453601092035301 & Nov. 1995-Nov. 1996 \\
\hline (Site 3) near Cumberland & 453612092034901 & Nov. 1995-Nov. 1996 \\
\hline (Site 4) near Cumberland & 453603092035701 & Nov. 1995-Nov. 1996 \\
\hline (Site 5) near Cumberland & 453608092041201 & Nov. 1995-Nov. 1996 \\
\hline (Site 6) near Cumberland & 453555092040901 & Nov. 1995-Nov. 1996 \\
\hline Lac La Belle, at Oconomowoc & 430733088305900 & $\begin{array}{l}\text { Feb. 1984-Aug. 1985, } \\
\text { Apr.-Aug. 1991, } \\
\text { Feb. 2001-Aug. } 2003\end{array}$ \\
\hline NW, at Oconomowoc & 430809088313900 & Feb. 1984-Aug. 1985 \\
\hline SE, at Oconomowoc & 430707088301400 & Feb. 1984-Aug. 1985 \\
\hline Lake Bastine, Deep Hole, near Mercer & 460511090153800 & Apr. 2009-Mar. 2010 \\
\hline Lake Blass, at Lake Delton & 433545089482400 & Mar. 1989-Aug. 1990 \\
\hline Lake Desair, near Rice Lake & 453446091465100 & Aug. 2004 \\
\hline
\end{tabular}


Table 1. Lake monitoring stations that were discontinued prior to 2013.—Continued

[NW, northwest; SE, southeast; SW, southwest; QW, water-quality station; (a), Wisconsin Valley Improvement Co. currently collects stage data for this site]

\begin{tabular}{|c|c|c|}
\hline Station name & Site identification number & Period of record \\
\hline East Bay, near Merton & 430957088183400 & Apr. 1991-Aug. 1995 \\
\hline North Bay, near Merton & 431006088191000 & Apr. 1991-Aug. 1995 \\
\hline Lake Morris at Mount Morris & 440654089120500 & Jun. 1983-Sept. 1989 \\
\hline $\begin{array}{l}\text { Lake Nebagamon, Northeast Bay, at Lake } \\
\text { Nebagamon }\end{array}$ & 463050091412300 & May 1992-Aug. 1995 \\
\hline Southeast Bay, at Lake Nebagamon & 462928091413500 & Mar. 1992-Sept. 1995 \\
\hline West Bay, at Lake Nebagamon & 463034091425300 & May 1992-Aug. 1995 \\
\hline Lake Noquebay, near Crivitz & 451511087550900 & $\begin{array}{l}\text { Feb. 1987-Aug. 1988, } \\
\text { Apr. 1991-Aug. } 1994\end{array}$ \\
\hline East End, near Crivitz & 451540087525700 & Apr. 1991-Aug. 1994 \\
\hline Lamotte Lake, near Shawano & 445305088361200 & Feb. 1990-Aug. 1992 \\
\hline $\begin{array}{l}\text { Lauderdale Lakes, at Lauderdale } \\
\text { Mill, at Lauderdale }\end{array}$ & $\begin{array}{l}424554088332700 \\
424555088335700\end{array}$ & $\begin{array}{l}\text { Oct. 1993-Oct. } 1994 \\
\text { Nov. 1993-Nov. 1994, } \\
\text { Aug. } 2002\end{array}$ \\
\hline Green, Auxiliary, Number 1, near Lauderdale & 424640088341900 & June 1999-Sept. 2000 \\
\hline Green, near Lauderdale & 424652088341500 & $\begin{array}{l}\text { Nov. 1993-Nov. 1994, } \\
\text { Aug. } 2002\end{array}$ \\
\hline Legend Lake (Site 1), near Shawano & 445342088312700 & Feb. 1990-Feb. 1992 \\
\hline Little Arbor Vitae, near Woodruff & 455446089370300 & Feb. 1991-Sept. 2002 \\
\hline Little Cedar Lake, North Site, near West Bend & 432255088134700 & $\begin{array}{l}\text { Feb. 1997-Aug. 1999, } \\
\text { Feb. 2003-Sept. } 2012\end{array}$ \\
\hline Little Cedar Lake, South Site, near West Bend & 432249088134500 & $\begin{array}{l}\text { Feb. 1997-Aug. 1999, } \\
\text { Feb. 2003-Sept. } 2012\end{array}$ \\
\hline Little Green Lake, at Center, near Markesan & 434412088590700 & Feb. 1991-Aug. 2003 \\
\hline Little Muskego Lake, at Muskego & 425425088083500 & Oct. 1986-Aug. 2002 \\
\hline Little Rock Lake, near Woodruff & 455946089415702 & Oct. 1983-Sept. 1996 \\
\hline Little St. Germain Lake, near Eagle River & 05390700 & (a) \\
\hline Upper East Bay, at St. Germain & 455532089253900 & $\begin{array}{l}\text { Dec. 1996-Mar. 97, } \\
\text { Mar. 1999, } \\
\text { Mar. 2000-Aug. } 2003\end{array}$ \\
\hline Northeast Bay, near St. Germain & 455545089262500 & $\begin{array}{l}\text { Apr. 1991-Aug. 1994, } \\
\text { Aug. 1996-Aug. 1997, } \\
\text { Mar. 1999-Aug. } 2003\end{array}$ \\
\hline South Bay, near St. Germain & 455437089270800 & $\begin{array}{l}\text { Apr. 1991-Aug. 1994, } \\
\text { Aug. 1996-Aug. 1997, } \\
\text { Mar. 1999-Aug. } 2003\end{array}$ \\
\hline West Bay, at St. Germain & 455428089282400 & $\begin{array}{l}\text { Apr. 1991-Aug. 1994, } \\
\text { Aug. 1996-Aug. 1997, } \\
\text { Mar. 1999-Aug. } 2003\end{array}$ \\
\hline Little Sand Lake (Site No. 2), near Mole Lake & 452826088544101 & May 1996-Sept. 2003 \\
\hline
\end{tabular}


Table 1. Lake monitoring stations that were discontinued prior to 2013.—Continued

[NW, northwest; SE, southeast; SW, southwest; QW, water-quality station; (a), Wisconsin Valley Improvement Co. currently collects stage data for this site]

\begin{tabular}{|c|c|c|}
\hline Station name & Site identification number & Period of re \\
\hline Long (Kee Nong Go-Mong) Lake, at Wind Lake & 424937088103400 & $\begin{array}{l}\text { Feb. 1988-Aug. 1989, } \\
\text { Feb. 1991-Aug. 1996 }\end{array}$ \\
\hline Loon Lake, near Shawano & 445009088303700 & Feb. 1991-Aug. 1993 \\
\hline Lost Lake, near Beaver Dam & 432640088580500 & June-Oct. 1991 \\
\hline \multicolumn{3}{|l|}{ McKenzie Lakes } \\
\hline \multicolumn{3}{|l|}{ McKenzie (Big McKenzie) } \\
\hline Deep Hole, near Spooner & 455507092013500 & Feb. 1987-Aug. 1998 \\
\hline Northern Site, near Spooner & 455540092022000 & June 1997-Aug. 1998 \\
\hline South Site, near Spooner & 455437092022300 & June 1997-Aug. 1998 \\
\hline Lower McKenzie, near Webb Lake & 455902092011900 & June 1997-Aug. 1998 \\
\hline Middle McKenzie, near Spooner & 455635092021800 & June 1997-Aug. 1998 \\
\hline Mary (Marie) Lake, at Twin Lakes & 423128088151200 & Feb. 1995-Aug. 1997 \\
\hline Max Lake, near Woodruff & 460128089423501 & Mar. 1988-Dec. 1996 \\
\hline Mead Lake, East Bay near Willard & 444720090445000 & Apr. 1991-Aug. 1995 \\
\hline West Bay, near Willard & 444733090460100 & Feb. 1991-Sept. 1995 \\
\hline Mercer Lake, Deep Hole, at Mercer & 460937090033100 & Mar. 2008-Sept. 2009 \\
\hline West basin, at Mercer & 460945090040600 & Mar. 2008-Sept. 2009 \\
\hline \multicolumn{3}{|l|}{ Minocqua Lake } \\
\hline Deep Hole, at Minocqua & 455214089412800 & May-Sept. 2003 \\
\hline North Bay, at Minocqua & 455232089424100 & May-Sept. 2003 \\
\hline South Bay, at Minocqua & 455206089425200 & May-Sept. 2003 \\
\hline Montello Lake, at Montello & 434748089195800 & Feb. 1995-Aug. 1998 \\
\hline Moon Lake, near St. Germain & 455504089260500 & Feb. 1992-Aug. 1996 \\
\hline Morgan Lake, near Fence & 454622088324801 & Oct. 1987-Sept. 1998. \\
\hline Moshawquit Lake, near Shawano & 445352088295800 & Feb. 1990-Aug. 1992 \\
\hline \multicolumn{3}{|l|}{ Muskego (Big Muskego) } \\
\hline Auxiliary Number 1, near Muskego & 425329088054000 & June 1996-Aug. 2000 \\
\hline Bass Bay, near Muskego & 425344008807010 & Feb. 1988-Aug. 2002 \\
\hline near Wind Lake & 425109088075000 & $\begin{array}{l}\text { Oct. 1987-Sept. 1989, } \\
\text { Jan. 1991-Sept. } 2002\end{array}$ \\
\hline South Site, near Muskego & 425212088072800 & Feb. 1988-Aug. 2002 \\
\hline Muskellunge Lake, near Eagle River & 455700089224900 & June 2000-Aug. 2001 \\
\hline $\begin{array}{l}\text { Muskellunge Lake, near Lake Outlet near Eagle } \\
\text { River }\end{array}$ & 455706089232400 & Nov. 2000-Oct. 2001 \\
\hline
\end{tabular}


Table 1. Lake monitoring stations that were discontinued prior to 2013.—Continued

[NW, northwest; SE, southeast; SW, southwest; QW, water-quality station; (a), Wisconsin Valley Improvement Co. currently collects stage data for this site]

\begin{tabular}{|c|c|c|}
\hline Station name & Site identification number & Period of record \\
\hline Nagawicka Lake, at Deep Hole, at Delafield & 430417088230300 & Feb. 2003-Sept. 2004 \\
\hline \multicolumn{3}{|l|}{ Namekagon Lakes } \\
\hline Garden, near Cable & 461224091033200 & Mar. 1998-Aug. 1999 \\
\hline Jackson, near Cable & 461457091065900 & Mar. 1998-Aug. 1999 \\
\hline \multicolumn{3}{|l|}{ Namekagon } \\
\hline Deep Hole, near Cable & 461308091065100 & Mar. 1998-Aug. 1999 \\
\hline East Basin, near Cable & 461228091044300 & Mar. 1998-Aug. 1999 \\
\hline Northeast Basin, near Cable & 461410091050700 & Mar. 1998-Aug. 1999 \\
\hline Park Lake (Site 1), at Pardeeville & 433239089175800 & $\begin{array}{l}\text { Feb. 1986-Aug. 1987, } \\
\text { May-Nov. } 1993\end{array}$ \\
\hline (Site 2) at Pardeeville & 433226089175500 & May-Nov. 1993 \\
\hline (Site 3) at Pardeeville & 433245089173000 & May-Nov. 1993 \\
\hline (Site 4) at Pardeeville & 433257089165100 & May-Nov. 1993 \\
\hline Pike Lake, near Hartford & 431916088200501 & Dec. 1998-Dec. 2000 \\
\hline Pike Lake-QW site, near Hartford & 431835088200600 & Feb.-Aug. 2000 \\
\hline Potter Lake, near Mukwonago & 424905088204000 & Feb. 1993-Sept. 2007 \\
\hline Pretty Lake, at Deep Hole, near Dousman & 425722088295000 & Feb. 1993-Aug. 1997 \\
\hline Puckaway Lake, West Basin, near Marquette & 434515089124000 & Apr. 2005-Sept. 2007 \\
\hline East Basin, near Marquette & 43454208907300 & Apr. 2005-Sept. 2007 \\
\hline River site, near Marquette & 434824089083200 & Apr. 2005-Sept. 2007 \\
\hline Red Cedar Lake, at Mikana & 453522091360600 & $\begin{array}{l}\text { Mar. 1993-Aug. 1994, } \\
\text { Mar. 1996-Aug. 1997, } \\
\text { Oct. 2000-Sept. } 2001\end{array}$ \\
\hline Deep Hole, near Mikana & 453725091345100 & $\begin{array}{l}\text { Mar. 1993-Aug. 1994, } \\
\text { Mar. 1996-Aug. 1997, } \\
\text { Mar. -Sept. } 2001\end{array}$ \\
\hline South End, at Mikana & 453519091352500 & $\begin{array}{l}\text { Mar. 1993-Aug. 1994, } \\
\text { Mar. 1996-Aug. 1997, } \\
\text { Mar. -Sept. } 2001\end{array}$ \\
\hline Rice Lake, at Deep Hole near Whitewater & 424629088415700 & Apr.-Nov. 1991 \\
\hline Round Lake, near Shawano & 445328088335000 & Feb. 1990-Aug. 1992 \\
\hline Sand Lake (Deep Hole), near Keshena & 445321088323101 & June-Aug. 1992 \\
\hline Shell Lake, at Shell Lake & 05334000 & Aug. 1936-Sept. 1999 \\
\hline Silver Lake, near Oconomowoc & 430436088293300 & Apr. 1992-Aug. 1996 \\
\hline Silver Lake, near West Bend & 432322088125000 & $\begin{array}{l}\text { Feb. 1996-Aug. } 1997 \\
\text { Feb. 2009-Aug. } 2009\end{array}$ \\
\hline
\end{tabular}


Table 1. Lake monitoring stations that were discontinued prior to 2013.—Continued

[NW, northwest; SE, southeast; SW, southwest; QW, water-quality station; (a), Wisconsin Valley Improvement Co. currently collects stage data for this site]

\begin{tabular}{|c|c|c|}
\hline Station name & Site identification number & Period of record \\
\hline $\begin{array}{l}\text { Sinissippi Lake, off Anthony Island., at } \\
\text { Hustisford }\end{array}$ & 432113088361100 & Feb. 1991-Aug. 1993 \\
\hline off Butternut Island., near Hustisford & 432240088363900 & Apr. 1991-Aug. 1993 \\
\hline off Sam Point, near Hustisford & 432300088374200 & Apr. 1991-Aug. 1993 \\
\hline Spirit Lake, near Keshena & 445400088320100 & Apr.-Aug. 1992 \\
\hline Spooner Lake, Deep Hole, near Spooner & 455034091493300 & June 2002-Aug. 2004 \\
\hline Southeast Site, near Spooner & 454945091483900 & June 2002-Aug. 2004 \\
\hline Stewart Lake, at Mt. Horeb & 430117089442701 & May 1992-Sept. 1993 \\
\hline Tichigan Lake, near Waterford & 424854088123300 & $\begin{array}{l}\text { Mar. 1994-Aug. 1996, } \\
\text { Apr. 2003-Aug. } 2004\end{array}$ \\
\hline Tombeau Lake, near Powers Lake & 423153088184800 & May 1998-Aug. 2000 \\
\hline Townline Lake, near Mercer & 460409090084100 & Apr. 2009-Mar. 2010 \\
\hline Trude Lake, Deep Hole, near Mercer & 460646090091900 & Apr. 2009-Mar. 2010 \\
\hline $\begin{array}{l}\text { Turtle-Flambeau Flowage, Deep Hole, near } \\
\text { Mercer }\end{array}$ & 460458090102700 & Apr. 2009-Mar. 2010 \\
\hline SW Basin, near Mercer & 460344090124800 & Apr. 2009-Mar. 2010 \\
\hline Lake Bastine, Deep Hole, near Mercer & 460511090153800 & Apr. 2009-Mar. 2010 \\
\hline Townline Lake, near Mercer & 460409090084100 & Apr. 2009-Mar. 2010 \\
\hline Trude Lake, Deep Hole, near Mercer & 460646090091900 & Apr. 2009-Mar. 2010 \\
\hline Twin Lake, East Twin, near Westfield & 435430089350700 & June 2002-Aug. 2004 \\
\hline West Twin, near Westfield & 435438089352300 & June 2002-Aug. 2004 \\
\hline Winnebago, Buoy Site, near Oshkosh & 440128088271201 & May 2011-Sept. 2011 \\
\hline
\end{tabular}

\section{Methods of Data Collection}

Depth profiles of water temperature, dissolved oxygen, $\mathrm{pH}$, and specific conductance were collected using multiparameter meters. Prior to measurements, the meters were calibrated using standards for $\mathrm{pH}$ and conductance, and dissolved oxygen was calibrated using the air calibration method. Generally, field measurements in profiles were made at 0.5-meter (m) intervals if the maximum depth of the lake was $5 \mathrm{~m}$ or less and at 1.0-m intervals if the maximum depth was greater than $5 \mathrm{~m}$. 
In most lakes, water samples were collected at two depths: near the surface and near the bottom. The chemistry of the water samples was analyzed using standard analytical methods by either the USGS National Water Quality Laboratory (Wershaw and others, 1987; Fishman and Friedman, 1989; Fishman, 1993) or the Wisconsin State Laboratory of Hygiene (Wisconsin State Laboratory of Hygiene, 1993). Analyses for dissolved constituents were done on samples that were filtered in the field through a filter with a pore size of 0.45 -micrometer. Total or total recoverable constituents were determined by analyzing unfiltered water samples. Preservation and shipment of samples followed standard protocols established by the laboratories. Waterquality data were archived in the Water Quality Data Base of the National Water Information System. Additional descriptive information about water-quality data is available at http://waterdata.usgs.gov/wi/nwis/qw. National Water Information System parameter codes and minimum laboratory reporting levels for chemical constituents are given in table 2. The parameter code for turbidity has changed from 00076 to 63675 or 63676 , because the method of testing has changed.

Records of lake stage are considered complete when one or more manual or automatic measurements were obtained per day. Partial records of lake-stage result when measurements were less frequent than daily. A complete description of manual or automatic measurements of lake stage is described by Rantz and others (1982).

Table 2. Parameter identification numbers and laboratory reporting levels for chemical parameters commonly measured in lakes, and analyzed at the National Water Quality Laboratory or the Wisconsin State Laboratory of Hygiene.

[NWQL, National Water Quality Laboratory; WSLH, Wisconsin State Laboratory of Hygiene; CAS, Chemical Abstracting Servce; LRL, laboratory reporting level; diss., dissolved; mg/L, milligram per liter; $\mu$ g/L, microgram per liter; —, not applicable; EPA, Environmental Protection Agency] 


\begin{tabular}{|c|c|c|c|c|c|c|c|c|c|}
\hline \multirow[b]{3}{*}{ Parameter name } & \multirow[b]{3}{*}{ Unit } & \multirow[b]{3}{*}{$\begin{array}{c}\text { CAS } \\
\text { number }{ }^{1}\end{array}$} & \multirow[b]{3}{*}{$\begin{array}{c}\text { Parameter } \\
\text { code }^{2}\end{array}$} & \multicolumn{4}{|c|}{ NWQL } & \multirow{2}{*}{\multicolumn{2}{|c|}{ WSLH }} \\
\hline & & & & \multicolumn{2}{|c|}{ Standard analysis } & \multicolumn{2}{|c|}{ Low-level analysis } & & \\
\hline & & & & LRL & Lab code & LRL & $\begin{array}{l}\text { Lab } \\
\text { code }\end{array}$ & LRL & Test code \\
\hline $\begin{array}{l}\text { Calcium, diss. } \\
\text { (Ca) }\end{array}$ & $\mathrm{mg} / \mathrm{L}$ & $7440-70-2$ & 00915 & 0.022 & 659 & - & - & 0.100 & E200.7 \\
\hline $\begin{array}{l}\text { Magnesium, diss. } \\
\text { (Mg) }\end{array}$ & $\mathrm{mg} / \mathrm{L}$ & 7439-95-4 & 00925 & 0.011 & 663 & - & - & 0.100 & E200.7 \\
\hline Sodium, diss. (Na) & $\mathrm{mg} / \mathrm{L}$ & $7440-23-5$ & 00930 & 0.06 & 675 & - & - & 0.100 & E200.7 \\
\hline $\begin{array}{l}\text { Potassium, diss. } \\
\text { (K) }\end{array}$ & $\mathrm{mg} / \mathrm{L}$ & 7440-09-7 & 00935 & 0.03 & 2773 & 0.004 & 2774 & 0.100 & E200.7 \\
\hline $\begin{array}{l}\text { Sulfate, diss. } \\
\quad\left(\mathrm{SO}_{4}\right)\end{array}$ & $\mathrm{mg} / \mathrm{L}$ & 14808-79-8 & 00945 & 0.02 & 1572 & 0.02 & 1263 & 4.70 & EPA 375.2 \\
\hline $\begin{array}{l}\text { Chloride, diss. } \\
\text { (Cl) }\end{array}$ & $\mathrm{mg} / \mathrm{L}$ & $16887-00-6$ & 00940 & 0.02 & 1571 & 0.02 & 1259 & 1.00 & SM4500-CL-E \\
\hline Fluoride, diss. (F) & $\mathrm{mg} / \mathrm{L}$ & 16984-48-8 & 00950 & 0.01 & 651 & 0.01 & 1260 & - & - \\
\hline Iron, diss. (Fe) & & 7439-89-6 & 01046 & 4.0 & 645 & - & - & 0.100 & E200.7 \\
\hline $\begin{array}{l}\text { Manganese, diss. } \\
\text { (Mn) }\end{array}$ & & 7439-96-5 & 01056 & 0.2 & 648 & 0.40 & 1793 & 1.00 & E200.7 \\
\hline Silica, diss. $\left(\mathrm{SiO}_{2}\right)$ & $\mathrm{mg} / \mathrm{L}$ & 7631-86-9 & 00955 & 0.06 & 3121 & 0.018 & 667 & 0.022 & $\begin{array}{l}\text { USGS I-2700- } \\
85\end{array}$ \\
\hline $\begin{array}{l}\text { Nitrogen, } \\
\quad \mathrm{NO}_{2}+\mathrm{NO}_{3} \text {, diss. }\end{array}$ & $\mathrm{mg} / \mathrm{L}$ & - & 00631 & 0.04 & 3156 & - & - & 0.019 & EPA 353.2 \\
\hline $\begin{array}{l}\text { Nitrogen, } \\
\text { ammonia, diss. }\end{array}$ & $\mathrm{mg} / \mathrm{L}$ & $7664-41-7$ & 00608 & 0.02 & 1991 & -- & -- & 0.015 & EPA 350.1 \\
\hline $\begin{array}{l}\text { Nitrogen, org., } \\
\text { total }^{3}\end{array}$ & $\mathrm{mg} / \mathrm{L}$ & - & - & - & - & - & - & - & - \\
\hline $\begin{array}{l}\text { Nitrogen, } \\
\text { amm.+org., } \\
\text { total }^{4}\end{array}$ & $\mathrm{mg} / \mathrm{L}$ & $17778-88-0$ & 00625 & 0.07 & 1986 & - & - & 0.14 & EPA 351.2 \\
\hline $\begin{array}{l}\text { Nitrogen, } \\
\text { amm.+org., } \\
\text { diss. }\end{array}$ & $\mathrm{mg} / \mathrm{L}$ & 17778-88-0 & 00623 & 0.07 & 1985 & - & - & - & - \\
\hline Phosphorus, total & $\mathrm{mg} / \mathrm{L}$ & 7723-14-0 & 00665 & 0.004 & 1984 & 0.004 & 2333 & 0.005 & EPA 365.1 \\
\hline $\begin{array}{l}\text { Phosphorus, ortho, } \\
\text { diss. }\end{array}$ & $\mathrm{mg} / \mathrm{L}$ & $14265-44-2$ & 00671 & 0.004 & 3118 & - & - & 0.002 & SM4500-PE \\
\hline $\begin{array}{l}\text { Chlorophyll } a \text {, } \\
\text { phytoplankton }\end{array}$ & $\mu \mathrm{g} / \mathrm{L}$ & 479-61-8 & 70953 & 0.1 & 3152 & - & - & - & - \\
\hline $\begin{array}{l}\text { Chlorophyll } a \text {, } \\
\text { phytoplankton }\end{array}$ & $\mu \mathrm{g} / \mathrm{L}$ & 479-61-8 & 32210 & - & - & - & - & 0.26 & EPA 445 \\
\hline
\end{tabular}

${ }^{1 .}$ CAS number-A unique identification for each constituent.

${ }^{2}$ Parameter code- $-\mathrm{A}$ unique number for storage of data in database.

${ }^{3 .}$ Calculated as difference between total ammonia + organic nitrogen and ammonia nitrogen

4.Also known as total Kjeldahl nitrogen (TKN).

\section{Explanation of Physical and Chemical Characteristics of Lakes}

Following are brief, generalized explanations of some of the common measurements of water quality and some of the physical processes occurring in lakes that influence these measures 
of water quality. More detailed explanations of water-quality data and lake processes are given by Wetzel (1983), Hem (1985), and Shaw and others (1993).

\section{Water Temperature and Thermal Stratification}

Water temperature in lakes is important because of its role in stratification and because of the temperature dependence of many chemical reactions and life processes of aquatic organisms. The extent of thermal stratification in lakes depends on the interaction between the lake's shape, water clarity, solar heating, and wind-driven mixing. Complete mixing of the lake is usually inhibited by thermal stratification in summer and by ice cover in winter. Thermal stratification affects water quality and the distribution of organisms in the lake. Summer thermal stratification can occur in any lake, but in Wisconsin it commonly occurs in lakes deeper than about $6 \mathrm{~m}$ (Shaw and others, 1993).

The density of water increases with decreasing temperature down to a temperature of 4 degrees Celsius $\left({ }^{\circ} \mathrm{C}\right)$, then decreases with decreasing temperature between $4{ }^{\circ} \mathrm{C}$ and the freezing point of water $\left(0^{\circ} \mathrm{C}\right)$. For a brief period in the spring after the ice is out, water temperature is usually uniform through the entire water column, and wind action causes the lake to mix completely. This process is known as “spring turnover.” As the lake absorbs the Sun’s energy, the surface water becomes warmer and its density decreases, making it more resistant to complete mixing. The difference in density caused by different water temperatures can prevent warm- and coldwater from mixing. In most lakes, therefore, a density "barrier” forms between the warmer surface water (epilimnion) and the underlying colder water (hypolimnion). This barrier is often marked by a sharp temperature gradient known as the "thermocline" (metalimnion). During the stratified summer period, these three distinct layers of lake water are often present. As the temperature difference between surface and deep water increases, this “stratified” condition stabilizes and can persist until surface temperatures decrease in the fall, 
which decreases the stability of the stratification. The mixing of the lake water in the fall is known as "fall turnover."

Thermal stratification may also occur under ice cover in the winter. In the winter, the coldest water (near $0{ }^{\circ} \mathrm{C}$ ) under the ice at the surface of the lake is less dense than water deeper in the lake with warmer temperatures.

\section{Specific Conductance}

Specific conductance is a measure of the ability of water to conduct an electrical current and is an indicator of the concentration of dissolved solids in the water. Because conductance is temperature related, reported values are normalized at $25^{\circ} \mathrm{C}$ and are termed "specific conductance.” As the concentration of dissolved minerals increases, specific conductance increases. During winter and summer thermal stratification, concentrations of dissolved constituents near the lake bottom increase due to one of two processes: the decomposition of materials settling from the epilimnion or the release of dissolved materials (such as iron, manganese, and phosphorus) from the bottom sediments during anoxic periods. Therefore, differences in specific conductance with depth indicate differences in concentrations of dissolved solids.

\section{Water Clarity}

Water clarity, or transparency, is commonly measured using a Secchi disc. The range of depths within which photosynthetic activity occurs depends largely on depth of light penetration, which is influenced by water clarity. A Secchi disc, most commonly a 20-centimeter diameter disc with alternating black-and-white quadrants, is lowered to a depth at which it is no longer visible. This depth is referred to as the "Secchi depth.” Clarity can be reduced by algae, zooplankton, water color, and suspended sediment. Algae are often the most dominant influence 
on clarity in lakes and, therefore, Secchi depth is usually correlated with the algal abundance. Secchi depths are generally the least during summer when algal populations are largest.

\section{$\mathrm{pH}$}

The $\mathrm{pH}$ is a measure of the acidity of the water. It is defined as the negative logarithm of hydrogen-ion concentration and varies over a 14-unit log scale, with a pH of 7 being neutral. Values less than 7 indicate acidic conditions; the lower the value, the stronger the acidity. Values greater than 7 indicate alkaline conditions. The $\mathrm{pH}$ of water is influenced in part by photosynthesis and respiration of planktonic algae and aquatic plants. It is important because it affects the solubility of many chemical constituents and because aquatic organisms have limited pH tolerances. Planktonic algae and aquatic plants produce oxygen and consume carbon dioxide as they photosynthesize during daytime; they consume oxygen and produce carbon dioxide when they respire at night. Carbon dioxide combines with the water molecule to form carbonic acid; therefore respiration causes a decrease in $\mathrm{pH}$ at night, and photosynthesis during the day causes an increase in $\mathrm{pH}$. The result is a daily cycle in $\mathrm{pH}$. Because phytoplankton are usually concentrated in the near-surface water, changes in $\mathrm{pH}$ in the epilimnion are more extreme than in the hypolimnion, where less photosynthesis usually occurs.

Lakes having good fish populations and productivity generally have a $\mathrm{pH}$ between 6.7 and 8.2. Values of $\mathrm{pH}$ greater than 8.5 have been shown to cause the release of phosphorus from lake sediments (James and Barko, 1991).

\section{Dissolved Oxygen}

Dissolved oxygen is one of the most critical factors affecting a lake ecosystem because it is essential to most aquatic organisms, and it is involved in many chemical reactions. Very low dissolved oxygen concentrations can control some types of chemical reactions. The solubility of 
oxygen in water is inversely related to temperature-that is, oxygen solubility decreases as water temperature increases. This relation is important, because at warmer temperatures the metabolic rate of organisms increases but less oxygen is available for respiration. The primary sources of dissolved oxygen are from the air and from photosynthesis. The minimum dissolved oxygen concentration specified in national water-quality criteria for early life stages of warmwater aquatic life is 5.0 milligrams per liter (U.S. Environmental Protection Agency, 1986).

In early summer, if thermal stratification develops, the metalimnion restricts the surface supply of dissolved oxygen to the hypolimnion. The hypolimnion can become isolated from the atmosphere. Thus, as summer progresses, the dissolved oxygen concentration can decrease in response to decomposition of dead algae that settle from the epilimnion and in response to the biological and chemical oxygen demand of the sediments. The oxygen demand from these processes may completely deplete the oxygen (anoxia) in the water near the lake bottom. The oxygen depletion then progresses upward but usually is confined to the hypolimnion.

Anoxia in the hypolimnion is common in stratified eutrophic (nutrient-rich) lakes in Wisconsin. Complete anoxia, however, is often not detected because of limitations of the measurement equipment. During anoxic conditions, many aquatic organisms cannot survive, but many other species (primarily bacteria) actually function only in such conditions. Therefore, a shift from oxic to anoxic conditions produces a rapid and dramatic change in the biological community and chemical environment. Anoxia also can cause the release of phosphorus from the bottom sediments. This phosphorus then mixes throughout the water column during spring and fall turnover.

\section{Phosphorus}

Phosphorus is one of the essential nutrients for plant growth. High phosphorus concentrations can cause dense algal populations (blooms) and can therefore be a major cause of 
eutrophication in lakes. When phosphorus concentrations exceed 0.025 milligrams per liter at the time of spring turnover in lakes and reservoirs, these water bodies may occasionally experience excess or nuisance growth of algae or other aquatic plants (U.S. Environmental Protection Agency, 1986). In many regions of the country, including the upper Midwest, other nutrients, particularly nitrogen, tend to be in abundant supply. Phosphorus is often the nutrient in shortest supply, therefore limiting or controlling plant growth. About 90 percent of the lakes in Wisconsin are limited by phosphorus (Shaw and others, 1993). In water, dissolved orthophosphate is the part of total phosphorus that is most readily available for use by algae. Internal phosphorus recycling occurs in many lakes. Phosphorus used by algae, aquatic plants, fish, and zooplankton is stored within these organisms. As these organisms die and decompose, this phosphorus is returned to the lake water and sediments. Anoxia in the hypolimnion makes phosphorus more soluble, adding further to the release of phosphorus from the falling particles and the lake sediments. During spring and fall turnover the phosphorus, which was released from the bottom sediments into the hypolimnion during anoxia, is mixed throughout the lake. The phosphorus is then available for algal growth. These phenomena are part of the internal recycling processes of lakes.

\section{Nitrogen}

Nitrogen, like phosphorus, is an essential nutrient for plant and algal growth. Usually in Wisconsin lakes, nitrogen is in abundant supply from the atmosphere and other sources. If phosphorus is abundant relative to algal needs, nitrogen can become the limiting nutrient. In that case, algal blooms are more likely to be triggered by increases in nitrogen than by increases in phosphorus. Some blue-green algal species can fix nitrogen from the atmosphere (Wetzel, 1983). Therefore, in situations where other types of algae are excluded because of a shortage of 
nitrogen, the nitrogen-fixing blue-green algae have a competitive advantage and may be present in abundance.

Lakes with a nitrogen to phosphorus ratio larger than 15 to 1 near the surface may generally be considered phosphorus limited, a ratio from 10 to 1 to 15 to 1 indicates a transition situation, and a ratio smaller than 10 to 1 generally indicates nitrogen limitation. Total nitrogen is the sum of ammonia, organic nitrogen, and nitrate-plus-nitrite nitrogen. The near-surface concentration is commonly used to compute the total nitrogen to phosphorus ratio because most algal species grow near the lake surface.

\section{Chlorophyll a}

Chlorophyll $a$ is a photosynthetic pigment found in algae (Wetzel, 1983) and other green plants. Its concentration, therefore, is commonly used as a measure of the density of the algal population in a lake. Chlorophyll $a$ concentrations are generally highest during summer when algal populations are highest. Moderate populations of desirable algae are important in the food chain; however, excessive populations or algal blooms are undesirable. Algal blooms can cause taste and odor problems and limit light penetration needed to support growth of submerged aquatic plants. Certain species of blue-green algae can produce toxins (Rapavich and others, 1987).

\section{Classification of Lakes}

Two methods are commonly used to classify and evaluate Wisconsin lakes according to their water quality or trophic state: Lillie and Mason’s (1983) water-quality index and Carlson’s (1977) trophic state index (TSI). In previous USGS data reports, a modification of Carlson’s TSI for Wisconsin lakes by Lillie and others (1993) had been used; however, this approach did not properly classify oligotrophic and highly eutrophic lakes and, therefore, was discontinued. 
Lillie and Mason’s (1983) water-quality indices for Wisconsin lakes were developed based on summer measurements of total phosphorus and chlorophyll $a$ concentrations and Secchi depth from a random set of lakes in Wisconsin. These data were used to classify the lakes’ water quality (table 3).

Table 3. Classifications of water quality in Wisconsin lakes.

[From Lillie and Mason (1983); mg/L, milligram per liter; $\mu \mathrm{g} / \mathrm{L}$, microgram per liter; <, less than; >, greater than]

\begin{tabular}{lccc}
\hline \multicolumn{1}{c}{ Water-quality index } & $\begin{array}{c}\text { Total phosphorus range } \\
(\mathrm{mg} / \mathrm{L})\end{array}$ & Chlorophyll a range $(\mu \mathrm{g} / \mathrm{L})$ & $\begin{array}{c}\text { Water clarity range (Secchi } \\
\text { depth, in meters) }\end{array}$ \\
\hline "Excellent" & $<0.001$ & 1.0 & $>6.0$ \\
"Very good" & $0.001-0.009$ & $1.0-4.9$ & $3.0-6.0$ \\
"Good" & $0.010-0.029$ & $5.0-9.9$ & $2.0-2.9$ \\
"Fair" & $0.030-0.049$ & $10.0-14.9$ & $1.5-1.9$ \\
"Poor" & $0.050-0.149$ & $15.0-30.0$ & $1.0-1.4$ \\
"Very poor" & $>0.150$ & $>30.0$ & $<1.0$ \\
\hline
\end{tabular}

Carlson’s (1977) TSI approach to lake classification assigns numerical ranges to the three trophic conditions generally used to describe the wide range of lake water-quality conditions. Oligotrophic lakes are typically clear, algal populations and phosphorus concentrations are low, and the deepest water is likely to contain oxygen throughout the year. Mesotrophic lakes typically have a moderate supply of nutrients, experience moderate algal blooms, and have occasional oxygen depletions at depth. Eutrophic lakes are nutrient rich with relatively severe water-quality problems, such as frequent seasonal algal blooms, oxygen depletion in lower parts of the lakes, and poor clarity. When eutrophic conditions are very severe, the lake is considered hypereutrophic.

Carlson’s (1977) TSI values are also based on near-surface total phosphorus and chlorophyll $a$ concentrations, and Secchi depths. The indices were developed to place these three characteristics on similar scales to allow comparison of different lakes. TSI values based on phosphorus concentrations, Secchi depths, and chlorophyll $a$ concentrations typically are computed only for measurements collected during the open-water period.

TSI values for a lake can be calculated using the following equations (Carlson, 1977): 
$\operatorname{TSI}_{P}=4.15+14.42 \times(\ln [$ total phosphorus concentration $\mathrm{x} 1,000])$

$T S I_{S D}=60.0-14.41 \times(\ln$ Secchi depth $)$

$T S I_{C}=30.6+9.81 \times($ ln chlorophyll a concentration $)$

where:

$T S I_{P}$ is total phosphorus, in milligrams per liter,

$T S I_{S D}$ is Secchi depth, in meters, and

$T S I_{C}$ is chlorophyll $a$, in micrograms per liter.

The three main trophic conditions (Carlson, 1977) are defined with boundaries for total phosphorus, Secchi depth, and chlorophyll $a$ (table 4).

Table 4. Characteristics of the three main tropic conditions.

[From Carlson (1977); m, meter; mg/L; milligram per liter; $\mu \mathrm{g} / \mathrm{L}$, microgram per liter]

\begin{tabular}{lllll}
\hline \multicolumn{1}{c}{ Trophic level } & \multicolumn{1}{c}{$\begin{array}{c}\text { Trophic State } \\
\text { Index }\end{array}$} & \multicolumn{1}{c}{$\begin{array}{c}\text { Total phosphorus } \\
(\mathrm{mg} / \mathrm{L})\end{array}$} & \multicolumn{1}{c}{$\begin{array}{c}\text { Secchi depth } \\
(\mathrm{m})\end{array}$} & \multicolumn{1}{c}{$\begin{array}{c}\text { Chlorophyll a } \\
(\boldsymbol{\mu} \mathrm{g} / \mathrm{L})\end{array}$} \\
\hline Eutrophic & $>50$ & $>0.024$ & $<2.0$ & $>7.2$ \\
Mesotrophic & $40-50$ & $0.012-0.024$ & $2.0-4.0$ & $2.6-7.2$ \\
Oligotrophic & $<40$ & $<0.012$ & $>4.0$ & $<2.6$ \\
\hline
\end{tabular}




\section{Acknowledgments}

This report is the culmination of a concerted effort by a number of people who collected, compiled, analyzed, verified, and organized the data, and who typed, edited, and assembled the report. The authors had primary responsibility for assuring that the information contained herein is accurate, complete, and adheres to USGS policy and established guidelines. Technicians in charge of the field offices are: B.W. Olson (Rhinelander), and S.A. March (Middleton). The data were collected and processed by D.D. Baumann, E.D. Dantoin, D.E. Housner, D.L. Olson, Z.T. Scott, B.J. Siebers and C.R. Utzig. S.B. Manteufel assembled, edited, and formatted the report. Additional assistance in preparation of the report was provided by M.M. Greenwood and D.L. Olson.

\section{References Cited}

Carlson, R.E., 1977, A trophic state index for lakes: Limnology and Oceanography, March, v. 22, no. 2, p. 361-369.

Fishman, M.J., ed., 1993, Methods of analysis by the U.S. Geological Survey National Water Quality Laboratory—Determination of inorganic and organic constituents in water and fluvial sediments: U.S. Geological Survey Open-File Report 93-125, 217 p.

Fishman, M.J., and Friedman, L.C., eds., 1989, Methods for determination of inorganic substances in water and fluvial sediments ( $3^{\text {rd }}$ ed.): U.S. Geological Survey Techniques of Water-Resources Investigations, book 5, chap. A1, 545 p.

Hem, J.D., 1985, Study and interpretation of the chemical characteristics of natural water ( $3^{\text {rd }}$ ed.): U.S. Geological Survey Water-Supply Paper 2254, 263 p.

James, W.F., and Barko, J.W., 1991, Littoral-pelagic phosphorus dynamics during nighttime convective circulation: Limnology and Oceanography, v. 36, no. 5, p. 946-960. 
Lillie, R.A., Graham, S., and Rasmussen, P., 1993, Trophic-state index equations and regional predictive equations for Wisconsin lakes: Wisconsin Department of Natural Resources Research Management Findings no. 35, 4 p.

Lillie, R.A., and Mason, J.W., 1983, Limnological characteristics of Wisconsin lakes: Wisconsin Department of Natural Resources Technical Bulletin no. 138, 116 p.

Rantz, S.E., and others, 1982, Measurement and computation of streamflow: U.S. Geological Survey Water-Supply Paper 2175, 631 p.

Rapavich, W.M., Sonzogni, W.C., Standridge, J.H., Vennie, J.G., and Wedepohl, R.E., 1987, Incidence of algal toxins in Wisconsin water experiencing blue-green algae blooms: Wisconsin State Laboratory of Hygiene and Wisconsin Department of Natural Resources, Informational Paper, 8 p.

Shaw, B., Mechenich, C., and Klessig, L., 2004, Understanding lake data: Madison, Wis., University of Wisconsin Extension, G3582, RP-03/2004, 19 p.

U.S. Environmental Protection Agency, 1986, Quality criteria for water 1986: U.S. Environmental Protection Agency publication, EPA 440/5-86-001 [variously paged].

Wershaw, R.L., Fishman, M.J., Grabbe, R.R., and Lowe, L.E., eds., 1987, Methods for the determination of organic substances in water and fluvial sediments: U.S. Geological Survey Techniques of Water-Resources Investigations, book 5, chap. A3, 80 p.

Wetzel, R.G., 1983, Limnology ( ${ }^{\text {nd }}$ ed.): New York, W.B. Saunders, 767 p.

Wisconsin State Laboratory of Hygiene, Environmental Sciences Section, 1993, Manual of analytical methods, inorganic chemistry unit: Wisconsin State Laboratory of Hygiene, revised November 1993 [variously paged]. 


\section{Lake Data}

Remarks codes and symbols used in the following tables:

$[<$, less than; $>$, greater than; --, not available; E, estimated] 


\section{ANVIL LAKE NEAR EAGLE RIVER, WI}

LOCATION.--Lat $45^{\circ} 57^{\prime} 00.5^{\prime \prime}$, long 8903'15.3", referenced to North American Datum of 1983, in NW 1/4 NE 1/4 Sec.13, T.40 N., R.11 E., Vilas County, Hydrologic Unit 07070001.

DRAINAGE AREA. $-4.11 \mathrm{mi}^{2}$

PERIOD OF RECORD.--June 2010 to current year.

GAGE.--Water-stage recorder and rain gage.

REMARKS.--Anvil Lake is a seepage lake. Gage height telemeter at station. Records are considered good.

EXTREMES FOR PERIOD OF RECORD.--Maximum, $97.20 \mathrm{ft}$, many days during May and June 1943; Minimum, $89.27 \mathrm{ft}$, Oct. 7 to 17, 2012.

EXTREMES FOR CURRENT YEAR.--Maximum, 90.44 ft, June 3; Minimum, 89.54 ft, Oct. 2, 2013.

GAGE HEIGHT, FEET

WATER YEAR OCTOBER 2013 TO SEPTEMBER 2014 DAILY MEAN VALUES

\begin{tabular}{|c|c|c|c|c|c|c|c|c|c|c|c|c|}
\hline DAY & OCT & NOV & DEC & JAN & FEB & MAR & APR & MAY & JUN & JUL & AUG & SEP \\
\hline 1 & 89.56 & 89.64 & 89.67 & 89.74 & 89.80 & 89.84 & 89.83 & 90.32 & 90.33 & 90.25 & 89.95 & 89.80 \\
\hline 2 & 89.54 & 89.64 & 89.67 & 89.74 & 89.80 & 89.84 & 89.83 & 90.32 & 90.43 & 90.23 & 89.94 & 89.81 \\
\hline 3 & 89.57 & 89.63 & 89.69 & 89.74 & 89.79 & 89.83 & 89.83 & 90.33 & 90.44 & 90.21 & 89.94 & 89.80 \\
\hline 4 & 89.58 & 89.63 & 89.72 & 89.75 & 89.79 & 89.84 & 89.89 & 90.34 & 90.42 & 90.20 & 89.94 & 89.96 \\
\hline 5 & 89.63 & 89.63 & 89.75 & 89.75 & 89.79 & 89.84 & 89.91 & 90.34 & 90.41 & 90.18 & 89.93 & 90.02 \\
\hline 6 & 89.69 & 89.66 & 89.75 & 89.75 & 89.79 & 89.83 & 89.90 & 90.34 & 90.40 & 90.18 & 89.92 & 90.01 \\
\hline 7 & 89.68 & 89.66 & 89.74 & 89.75 & 89.79 & 89.84 & 89.91 & 90.34 & 90.38 & 90.18 & 89.91 & 89.99 \\
\hline 8 & 89.67 & 89.65 & 89.74 & 89.74 & 89.78 & 89.84 & 89.91 & 90.35 & 90.36 & 90.22 & 89.89 & 89.98 \\
\hline 9 & 89.66 & 89.68 & 89.74 & 89.74 & 89.78 & 89.83 & 89.91 & 90.37 & 90.35 & 90.21 & 89.88 & 89.97 \\
\hline 10 & 89.65 & 89.67 & 89.74 & 89.74 & 89.78 & 89.83 & 89.92 & 90.36 & 90.33 & 90.19 & 89.87 & 90.06 \\
\hline 11 & 89.64 & 89.67 & 89.74 & 89.75 & 89.77 & 89.83 & 89.93 & 90.36 & 90.32 & 90.18 & 89.87 & 90.10 \\
\hline 12 & 89.63 & 89.66 & 89.74 & 89.75 & 89.78 & 89.82 & 89.96 & 90.39 & 90.31 & 90.16 & 89.87 & 90.09 \\
\hline 13 & 89.61 & 89.65 & 89.74 & 89.75 & 89.78 & 89.82 & 89.98 & 90.40 & 90.29 & 90.15 & 89.85 & 90.10 \\
\hline 14 & 89.60 & 89.65 & 89.73 & 89.75 & 89.79 & 89.82 & 90.02 & 90.39 & 90.27 & 90.15 & 89.83 & 90.08 \\
\hline 15 & 89.62 & 89.64 & 89.74 & 89.75 & 89.79 & 89.81 & 90.03 & 90.38 & 90.29 & 90.14 & 89.81 & 90.07 \\
\hline 16 & 89.64 & 89.65 & 89.74 & 89.76 & 89.80 & 89.81 & 90.04 & 90.37 & 90.29 & 90.13 & 89.80 & 90.06 \\
\hline 17 & 89.64 & 89.70 & 89.74 & 89.76 & 89.80 & 89.81 & 90.12 & 90.36 & 90.28 & 90.11 & 89.78 & 90.05 \\
\hline 18 & 89.64 & 89.70 & 89.73 & 89.76 & 89.81 & 89.82 & 90.13 & 90.35 & 90.26 & 90.10 & 89.80 & 90.03 \\
\hline 19 & 89.64 & 89.69 & 89.73 & 89.76 & 89.79 & 89.83 & 90.13 & 90.35 & 90.25 & 90.09 & 89.80 & 90.02 \\
\hline 20 & 89.64 & 89.69 & 89.74 & 89.76 & --- & 89.83 & 90.14 & 90.41 & 90.27 & 90.07 & 89.80 & 90.01 \\
\hline 21 & 89.65 & 89.70 & 89.73 & 89.76 & --- & 89.83 & 90.15 & 90.42 & 90.27 & 90.06 & 89.79 & 90.07 \\
\hline 22 & 89.65 & 89.70 & 89.74 & 89.76 & --- & 89.83 & 90.15 & 90.41 & 90.26 & 90.06 & 89.79 & 90.06 \\
\hline 23 & 89.65 & 89.69 & 89.74 & 89.76 & --- & 89.83 & 90.15 & 90.40 & 90.26 & 90.04 & 89.80 & 90.06 \\
\hline 24 & 89.65 & 89.69 & 89.74 & 89.76 & --- & 89.82 & 90.16 & 90.39 & 90.29 & 90.03 & 89.79 & 90.05 \\
\hline 25 & 89.65 & 89.68 & 89.75 & 89.78 & 89.83 & 89.82 & 90.21 & 90.38 & 90.28 & 90.02 & 89.81 & 90.06 \\
\hline 26 & 89.64 & 89.68 & 89.75 & 89.78 & 89.84 & 89.82 & 90.21 & 90.38 & 90.27 & 90.01 & 89.79 & 90.06 \\
\hline 27 & 89.64 & 89.68 & 89.75 & 89.79 & 89.85 & 89.82 & 90.21 & 90.37 & 90.26 & 90.00 & 89.78 & 90.05 \\
\hline 28 & 89.62 & 89.68 & 89.75 & 89.78 & 89.83 & 89.84 & 90.22 & 90.35 & 90.26 & 89.99 & 89.77 & 90.05 \\
\hline 29 & 89.62 & 89.68 & 89.75 & 89.78 & --- & 89.84 & 90.24 & 90.34 & 90.28 & 89.97 & 89.77 & 90.04 \\
\hline 30 & 89.62 & 89.68 & 89.75 & 89.79 & --- & 89.84 & 90.30 & 90.33 & 90.27 & 89.97 & 89.80 & 90.04 \\
\hline 31 & 89.63 & --- & 89.75 & 89.80 & -- & 89.83 & -- & 90.32 & --- & 89.97 & 89.79 & --- \\
\hline MEAN & 89.63 & 89.67 & 89.73 & 89.76 & --- & 89.83 & 90.04 & 90.36 & 90.31 & 90.11 & 89.84 & 90.02 \\
\hline MAX & 89.69 & 89.70 & 89.75 & 89.80 & --- & 89.84 & 90.30 & 90.42 & 90.44 & 90.25 & 89.95 & 90.10 \\
\hline MIN & 89.54 & 89.63 & 89.67 & 89.74 & --- & 89.81 & 89.83 & 90.32 & 90.25 & 89.97 & 89.77 & 89.80 \\
\hline
\end{tabular}




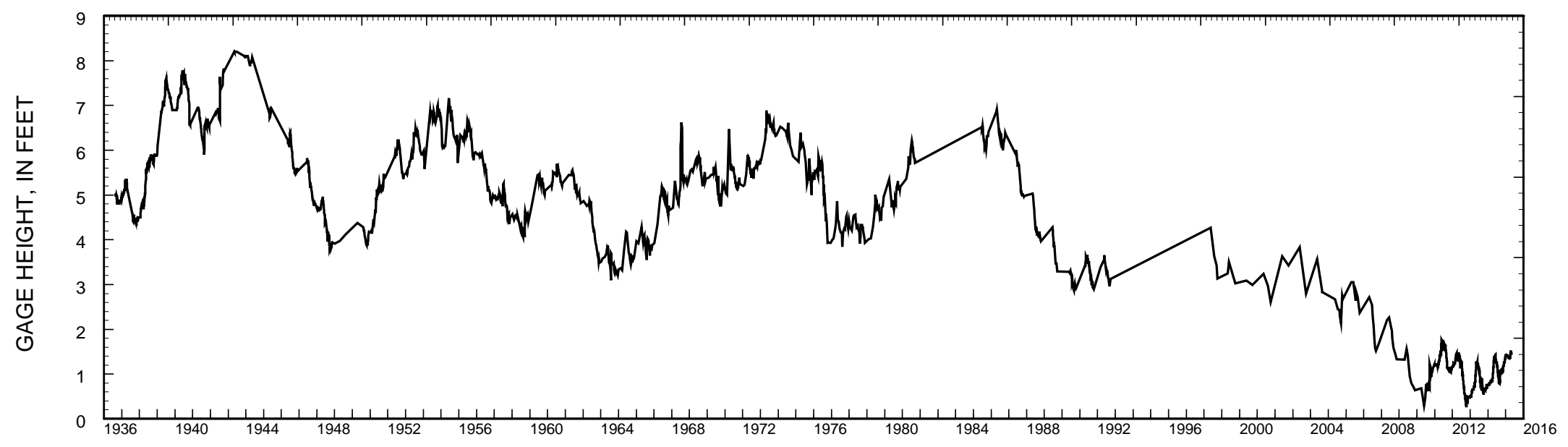

Stage hydrograph for Anvil Lake, 1936-2014. 


\section{ANVIL LAKE, DEEP HOLE, NEAR EAGLE RIVER, WI}

LOCATION.--Lat 4556'38", long 8903'45", in SW 1/4 Sec.13, T.40 N., R.11 E., Vilas County, Hydrologic Unit 07070001, near Eagle River.

SURFACE AREA. $-0.62 \mathrm{mi}^{2}$.

PERIOD OF RECORD.-May 2012 to September 2013.

REMARKS.--Lake sampled at the deep hole at a depth of 8-9 m. Water-quality analyses by Wisconsin State Laboratory of Hygiene. A "*" indicates data that were collected by a Citizen Lake Monitoring Volunteer.

WATER-QUALITY DATA, OCTOBER 21, 2013 TO SEPTEMBER 25, 2014

(Milligrams per liter unless otherwise indicated)

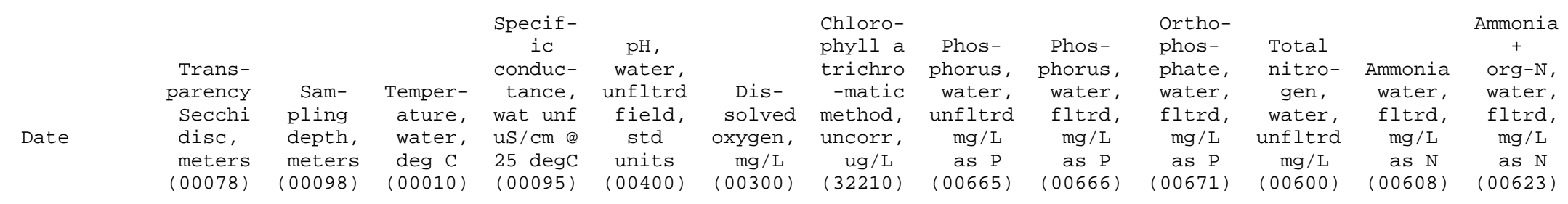

OCT 2013

$21 \ldots$

FEB 2014

$12 \ldots$

$\begin{array}{ccr}3.60 & -- & -- \\ -- & 0.50 & 11.2 \\ 2.60 & -- & -- \\ -- & 0.50 & 0.4 \\ 6.10 & -- & -- \\ -- & 2.0 & 2.4 \\ -- & 6.0 & 4.0 \\ -- & 7.5 & 4.4 \\ & & \\ 2.75 & -- & -- \\ -- & 0.50 & 8.4 \\ 4.70 & 0.50 & -- \\ & & \\ 5.00 & 0.50 & -- \\ 4.90 & 0.50 & -- \\ 4.20 & -- & -- \\ -- & 0.50 & 21.1 \\ -- & 8.0 & 11.6 \\ -- & 0.50 & --\end{array}$

$\begin{array}{crr}-- & -- & -- \\ 43 & 7.5 & 8.3 \\ -- & -- & -- \\ 45 & 7.4 & 12.4 \\ & & \\ -- & -- & -- \\ 43 & 7.2 & 8.8 \\ 47 & 6.5 & 1.1 \\ 53 & 6.4 & 0.2 \\ & & \\ -- & -- & -- \\ 40 & 6.7 & 10.5 \\ -- & -- & -- \\ & & \\ -- & -- & -- \\ -- & -- & -- \\ -- & -- & -- \\ 40 & 8.2 & 9.1 \\ 46 & 6.8 & 2.8 \\ -- & -- & --\end{array}$

$\begin{array}{cc}8 .- & -- \\ -31 & 0.017 \\ -- & -- \\ 1.95 & 0.019 \\ & \\ -- & -- \\ -- & 0.011 \\ -- & 0.014 \\ -- & 0.014 \\ & \\ -- & -- \\ 6.37 & 0.012 \\ -- & 0.010 \\ & \\ 1.27 & 0.013 \\ 3.89 & 0.016 \\ -- & -- \\ 3.05 & 0.009 \\ -- & 0.016 \\ 2.93 & 0.012\end{array}$


455638089034501 ANVIL LAKE, DEEP HOLE, NEAR EAGLE RIVER, WI

\begin{tabular}{|c|c|c|c|c|c|c|c|c|c|c|c|c|c|}
\hline & & & & WATER-C & $\begin{array}{l}\text { UALITY DAT } \\
\text { (Milligran }\end{array}$ & $\begin{array}{l}\text { A, OCTOBE } \\
\text { s per liter ur }\end{array}$ & $\begin{array}{l}\text { 21, } 2013 \mathrm{~T} \\
\text { less otherwis }\end{array}$ & $\begin{array}{l}\text { SEPTEMBE } \\
\text { indicated) }\end{array}$ & $R 25,2014$ & & & & \\
\hline Date & $\begin{array}{c}\text { Trans- } \\
\text { parency } \\
\text { Secchi } \\
\text { disc, } \\
\text { meters } \\
(0 \odot \odot 78)\end{array}$ & $\begin{array}{c}\text { Sam- } \\
\text { pling } \\
\text { depth, } \\
\text { meters } \\
(\odot \odot \odot 98)\end{array}$ & $\begin{array}{c}\text { Temper - } \\
\text { ature, } \\
\text { water, } \\
\text { deg C } \\
(00010)\end{array}$ & $\begin{array}{c}\text { Specif- } \\
\text { ic } \\
\text { conduc- } \\
\text { tance, } \\
\text { wat unf } \\
\text { uS/cm @ } \\
25 \mathrm{degc} \\
(\odot \odot \odot 95)\end{array}$ & $\begin{array}{l}\text { pH, } \\
\text { water, } \\
\text { unfltrd } \\
\text { field, } \\
\text { std } \\
\text { units } \\
(\odot \odot 4 \odot \odot)\end{array}$ & $\begin{array}{c}\text { Dis- } \\
\text { solved } \\
\text { oxygen, } \\
\mathrm{mg} / \mathrm{L} \\
(\odot \odot 30 \odot)\end{array}$ & $\begin{array}{c}\text { Chloro- } \\
\text { phyll a } \\
\text { trichro } \\
\text {-matic } \\
\text { method, } \\
\text { uncorr, } \\
\text { ug/L } \\
(32210)\end{array}$ & $\begin{array}{c}\text { Phos- } \\
\text { phorus, } \\
\text { water, } \\
\text { unfltrd } \\
\text { mg/L } \\
\text { as } P \\
(00665)\end{array}$ & $\begin{array}{c}\text { Phos- } \\
\text { phorus, } \\
\text { water, } \\
\text { fltrd, } \\
\text { mg/L } \\
\text { as } P \\
(00666)\end{array}$ & $\begin{array}{l}\text { ortho- } \\
\text { phos- } \\
\text { phate, } \\
\text { water, } \\
\text { fltrd, } \\
\text { mg/L } \\
\text { as P } \\
(00671)\end{array}$ & $\begin{array}{c}\text { Total } \\
\text { nitro- } \\
\text { gen, } \\
\text { water, } \\
\text { unfltrd } \\
\text { mg/L } \\
(\odot \odot 6 \odot \odot)\end{array}$ & $\begin{array}{c}\text { Ammonia } \\
\text { water, } \\
\text { fltrd, } \\
\text { mg/L } \\
\text { as N } \\
(00608)\end{array}$ & $\begin{array}{c}\text { Ammonia } \\
+ \\
\text { org-N, } \\
\text { water, } \\
\text { fltrd, } \\
\text { mg/L } \\
\text { as N } \\
(00623)\end{array}$ \\
\hline JUL & & & & & & & & & & & & & \\
\hline${ }^{*} 04 \ldots$ & 4.80 & 0.50 & -- & - - & - - & -- & 1.60 & 0.011 & -- & - - & -- & -- & -- \\
\hline $17 \ldots$ & - - & - - & - & - - & - - & - - & 3.37 & $\odot .025$ & - - & -- & - - & - - & - - \\
\hline$* 17 \ldots$ & 4.00 & 0.50 & - - & - - & - - & - - & 3.37 & 0.014 & - - & - - & - - & - - & - - \\
\hline $23 \ldots$ & 3.05 & - - & - - & - - & - - & - - & - - & - & - - & - - & - - & - - & - - \\
\hline $23 \ldots$ & - - & 0.50 & 21.9 & 43 & 7.6 & 8.8 & 4.82 & 0.013 & - - & $\odot . \odot \odot 4$ & $<0.35$ & $<0.015$ & 0.27 \\
\hline $23 \ldots$ & - - & 8.0 & 15.1 & 57 & 6.2 & 0.8 & - - & 0.024 & - - & - - & - - & - - & - - \\
\hline AUG & & & & & & & & & & & & & \\
\hline $08 \ldots$ & - - & - - & - - & - - & - - & - - & 6.44 & 0.016 & - - & - - & - - & - - & - - \\
\hline${ }^{*} \odot 8 \ldots$ & 3.00 & 0.50 & - - & - - & - - & - - & 1.38 & 0.013 & - - & - - & - - & - - & - - \\
\hline $26 \ldots$ & -- & - - & - - & -- & - - & - - & 9.25 & 0.018 & -- & -- & - - & -- & - - \\
\hline $26 \ldots$ & - - & 6.0 & 21.1 & 42 & 7.8 & 7.8 & - - & 0.019 & - - & - - & - - & - - & - - \\
\hline $26 \ldots$ & - - & 7.0 & 19.3 & 47 & 6.8 & 1.9 & - - & 0.019 & - - & - - & - - & -- & -. \\
\hline $26 \ldots$ & - - & 8.0 & 17.6 & 54 & 6.3 & 0.3 & - - & $\odot .039$ & - - & -- & - - & - - & - - \\
\hline $26 \ldots$ & 2.25 & - - & - - & - - & - - & - - & - - & - - & -- & - - & - - & - - & -- \\
\hline$* 26 \ldots$ & 2.70 & 0.50 & -- & -- & -- & - & 8.97 & $\odot .018$ & - - & - & - - & - & -- \\
\hline SEP & & & & & & & & & & & & & \\
\hline $25 \ldots$ & 2.20 & - - & - - & - - & - - & - - & - - & - - & -- & - - & - - & -- & - - \\
\hline $25 \ldots$ & -- & 0.50 & 16.3 & 40 & 8.3 & 10.1 & 13.7 & 0.018 & -- & -- & - & -- & -- \\
\hline $25 \ldots$ & - - & 8.0 & 15.2 & 39 & 7.4 & 7.9 & - - & 0.017 & - - & - - & - - & - - & -. \\
\hline
\end{tabular}




\section{ANVIL LAKE, DEEP HOLE, NEAR EAGLE RIVER, W}

\section{WATER-QUALITY DATA, OCTOBER 21, 2013 TO SEPTEMBER 25, 2014}

(Milligrams per liter unless otherwise indicated)

Ammonia Nitrate

$\stackrel{+}{+} \stackrel{+}{+}$

water, water, unfltrd fltrd,

Date

$$
\mathrm{mg} / \mathrm{L} \quad \mathrm{mg} / \mathrm{L}
$$

$(0 \odot 625) \quad(0 \odot 631)$

MAY

\begin{tabular}{|c|c|c|}
\hline $15 \ldots$ & - - & - \\
\hline $15 \ldots$ & 0.32 & $\odot .094$ \\
\hline $28 \ldots$ & - - & - - \\
\hline \multicolumn{3}{|l|}{ JUL } \\
\hline $23 \ldots$ & - - & - - \\
\hline $23 \ldots$ & 0.33 & $<0.019$ \\
\hline $23 \ldots$ & - - & - - \\
\hline \multicolumn{3}{|l|}{ AUG } \\
\hline $08 \ldots$ & - - & - - \\
\hline $08 \ldots$ & - - & - - \\
\hline $26 \ldots$ & -- & - - \\
\hline $26 \ldots$ & 0.61 & $<0,019$ \\
\hline $26 \ldots$ & - - & -- \\
\hline $26 \ldots$ & - - & - - \\
\hline $26 \ldots$ & - - & - - \\
\hline $26 \ldots$ & - - & - - \\
\hline $26 \ldots$ & - - & - - \\
\hline $26 \ldots$ & -- & - - \\
\hline \multicolumn{3}{|l|}{ SEP } \\
\hline $25 \ldots$ & -- & -- \\
\hline $25 \ldots$ & - - & - - \\
\hline $25 \ldots$ & - & \\
\hline
\end{tabular}


455638089034501 ANVIL LAKE, DEEP HOLE, NEAR EAGLE RIVER, WI

LAKE-DEPTH PROFILES, OCTOBER 21, 2013 TO MAY 14, 2014

$10-21-13$
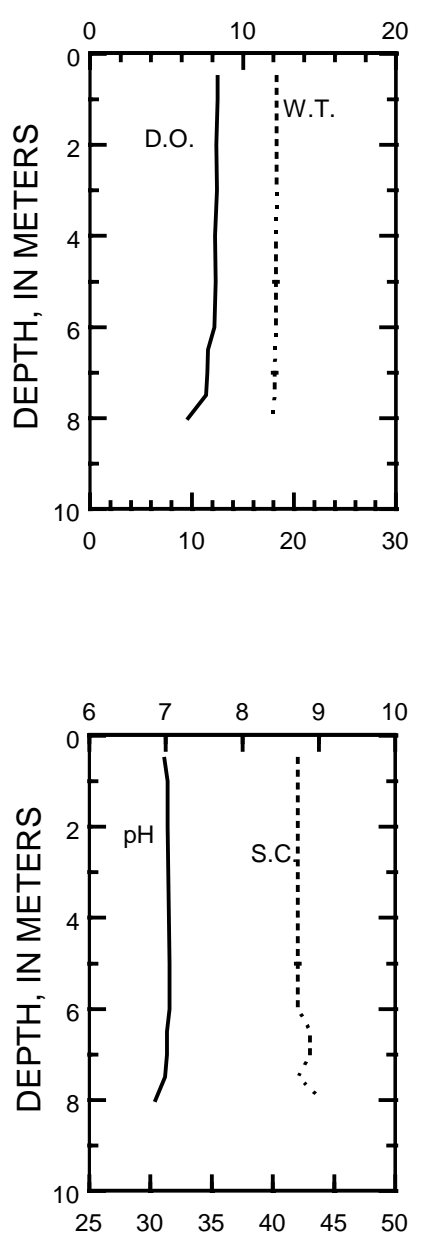

02-12-14

04-07-14

DISSOLVED OXYGEN (D.O.), IN MILLIGRAMS PER LITER
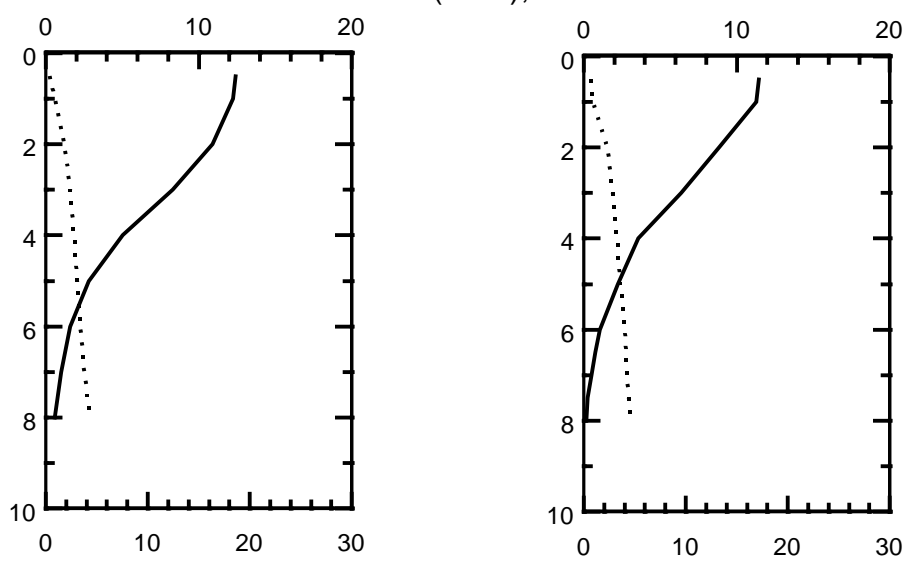

WATER TEMPERATURE (W.T.), IN DEGREES CELSIUS

$\mathrm{PH}$, IN STANDARD UNITS
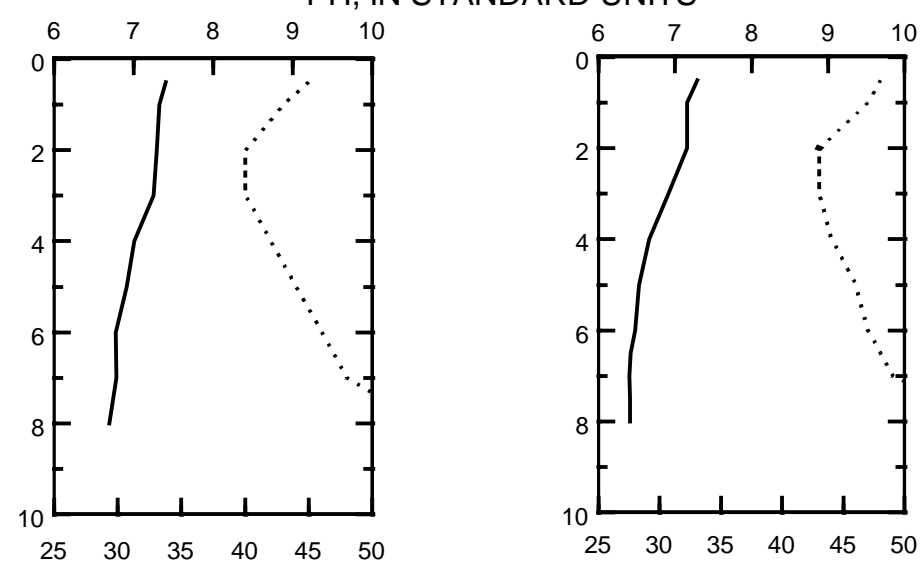

05-14-14
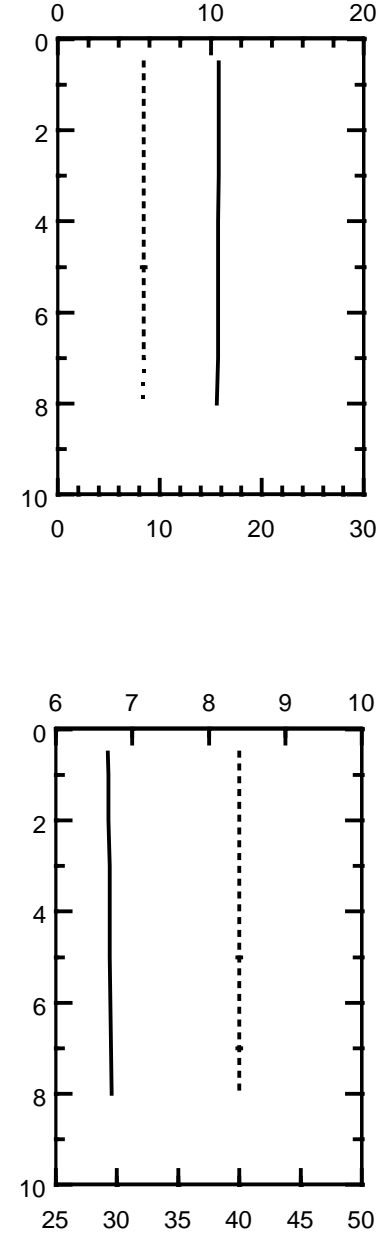

SPECIFIC CONDUCTANCE (S.C.), IN MICROSIEMENS PER CENTIMETER AT 25 DEGREES CELSIUS 
455638089034501 ANVIL LAKE, DEEP HOLE, NEAR EAGLE RIVER, WI

LAKE-DEPTH PROFILES, JUNE 23 TO SEPTEMBER 25, 2014

06-23-14
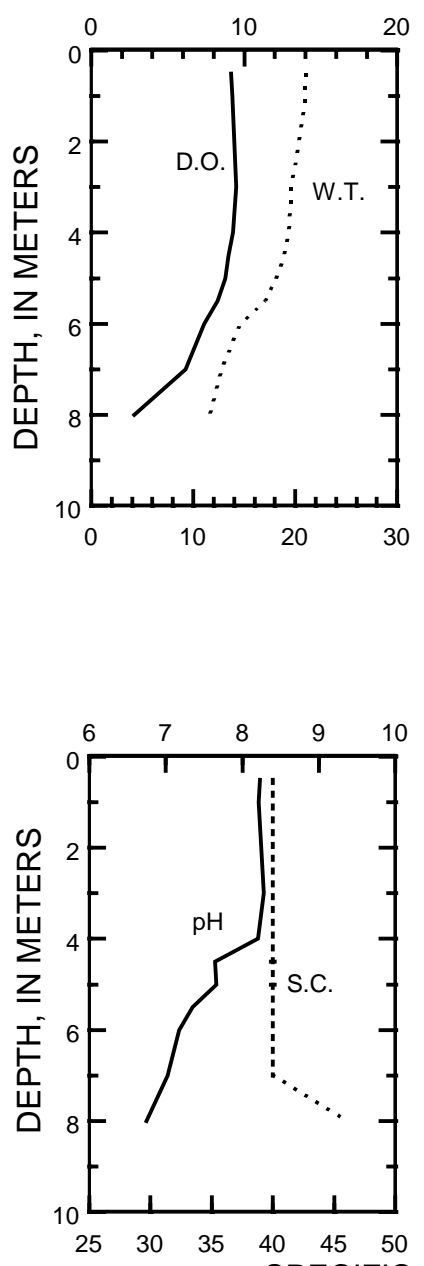

07-23-14 DISSOLVED OXYGEN (D.O.), IN MILLIGRAMS PER LITER

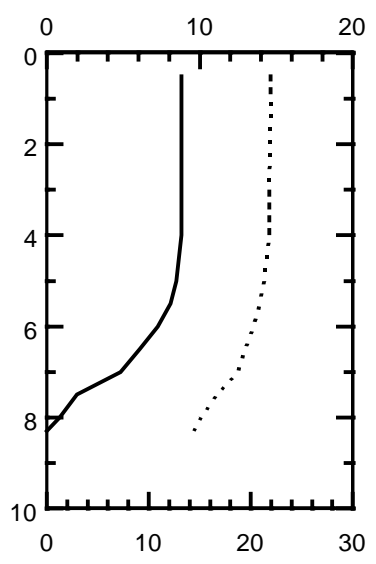

WATER TEMPERATURE (W.T.), IN DEGREES CELSIUS

$\mathrm{PH}$, IN STANDARD UNITS
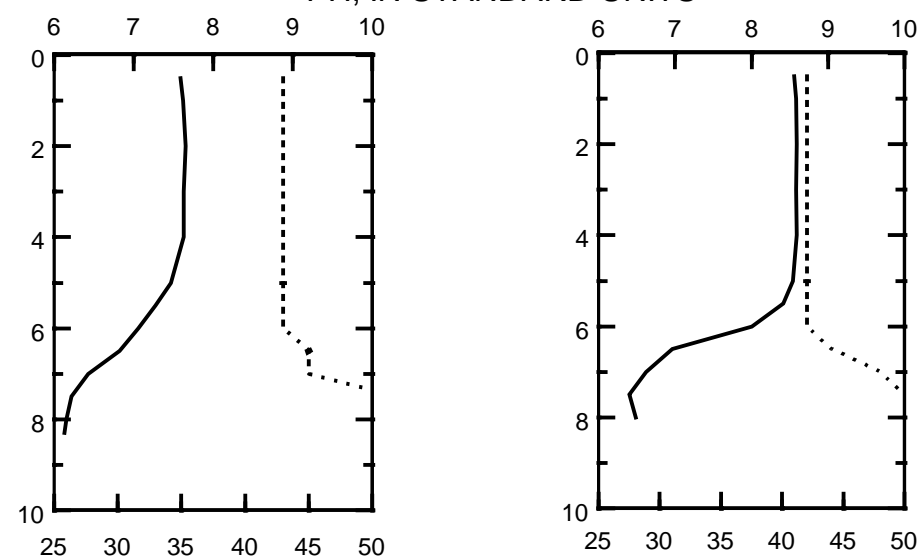

$09-25-14$
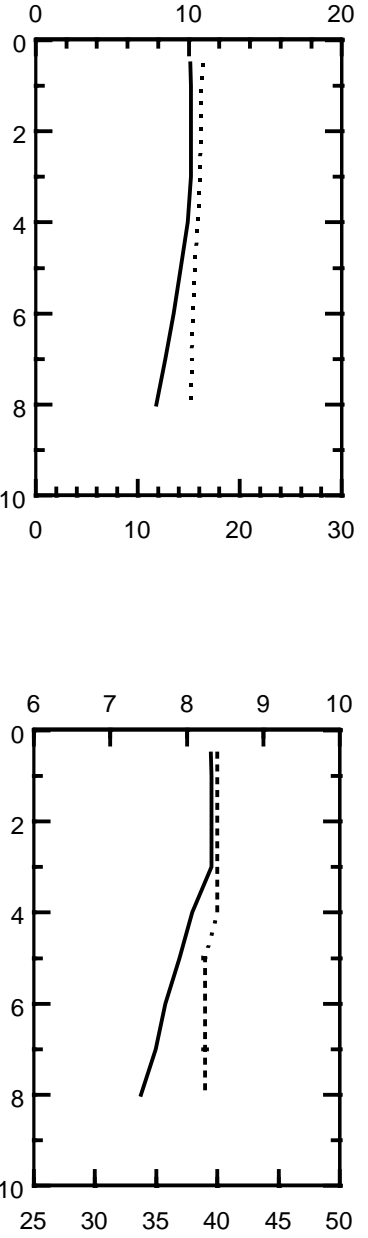

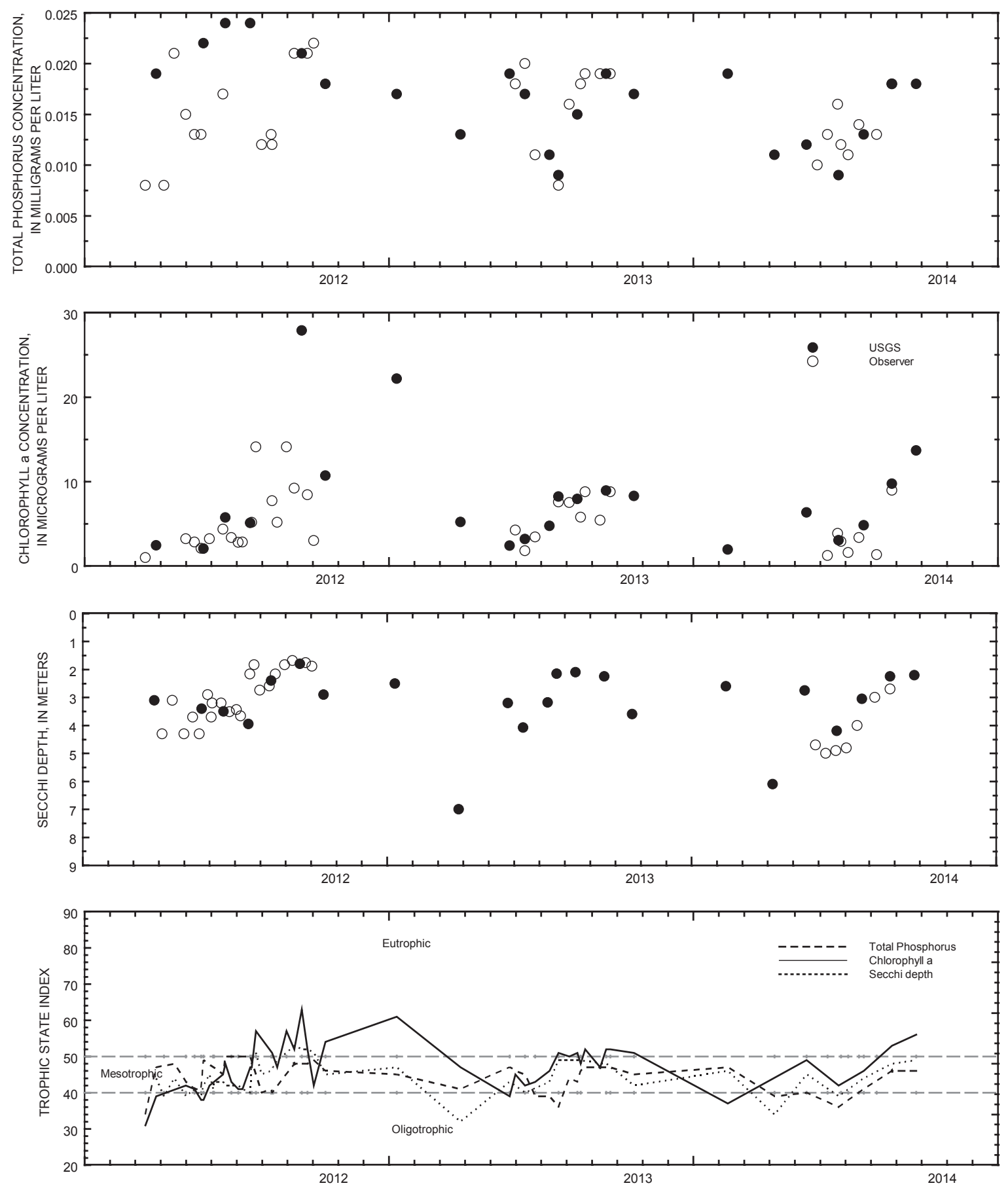

Surface total phosphorus, chlorophyll a concentrations, Secchi depths, and TSI data for Anvil Lake, Deep Hole, near Eagle River, Wisconsin. 


\section{LAKE BEULAH AT DEEP HOLE NEAR EAST TROY, WI}

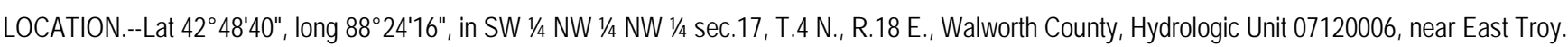

SURFACE AREA.--1.30 mi².

PERIOD OF RECORD.--August 2007 to August 2014.

REMARKS.--Lake sampled at the deep hole at a depth of $19 \mathrm{~m}$. Water-quality analyses by Wisconsin State Laboratory of Hygiene.

WATER-QUALITY DATA, MAY 13 TO AUGUST 27, 2014

(Milligrams per liter unless otherwise indicated)

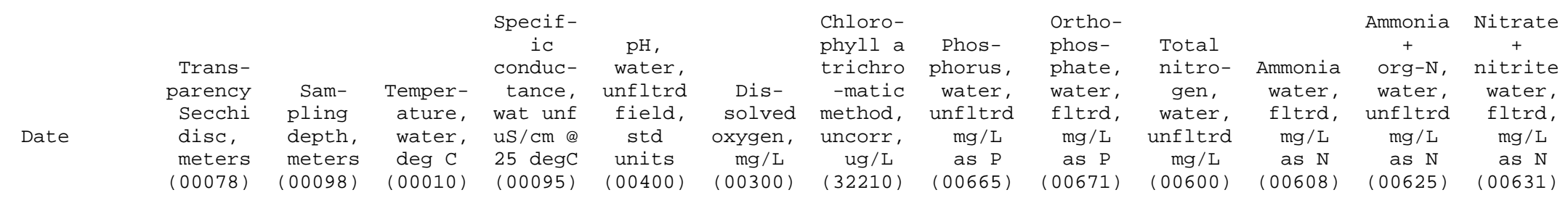

MAY 2014

$\begin{array}{ccrrrrr}13 \ldots & 5.45 & -- & -- & -- & -- & - \\ 13 \ldots & -- & 0.50 & 16.2 & 566 & 7.9 & 10.6 \\ \text { AUG } & & & & & & \\ 27 \ldots & 2.75 & -- & -- & -- & -- & - \\ 27 \ldots & -- & 2.0 & 24.7 & 500 & 8.5 & 8.6 \\ 27 \ldots & -- & 4.0 & 24.3 & 527 & 8.0 & 4.6 \\ 27 \ldots & -- & 11.0 & 7.7 & 614 & 7.6 & 0.4 \\ 27 \ldots & -- & 17.0 & 5.0 & 640 & 7.4 & 0.2 \\ 27 \ldots & -- & 18.0 & 4.9 & 651 & 7.4 & 0.2\end{array}$

$\begin{array}{ccc}-- & -- & -- \\ 1.60 & 0.013 & 0.009 \\ -- & -- & -- \\ 4.00 & 0.011 & 0.008 \\ -- & 0.016 & 0.009 \\ -- & 0.008 & 0.005 \\ -- & 0.024 & 0.006 \\ -- & 0.027 & 0.004\end{array}$

$\begin{array}{cccc}-- & -- & -- & -- \\ 1.2 & 0.050 & 0.49 & 0.730 \\ & & & \\ -- & -- & -- & -- \\ <0.55 & 0.030 & 0.53 & <0.020 \\ <0.57 & 0.060 & 0.55 & <0.020 \\ 1.2 & 0.190 & 0.53 & 0.650 \\ <1.1 & 0.590 & 1.1 & <0.020 \\ 1.9 & 1.15 & 1.9 & 0.060\end{array}$


424840088241600 LAKE BEULAH AT DEEP HOLE NEAR EAST TROY, WI

WATER-QUALITY DATA, MAY 13 TO AUGUST 27, 2014

(Milligrams per liter unless otherwise indicated)

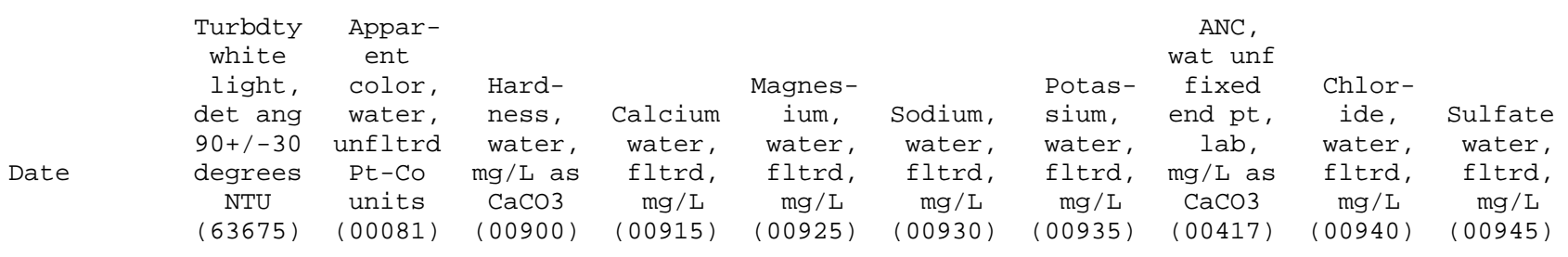

MAY 2014

\begin{tabular}{|c|c|c|c|c|c|c|c|c|c|c|}
\hline $13 \ldots$ & -- & - - & - - & - - & -- & - - & - - & - - & - - & - - \\
\hline $13 \ldots$ & 2.0 & 13 & 295 & 62.5 & 33.7 & 9.70 & 1.74 & 244 & 23.4 & 27.4 \\
\hline \multicolumn{11}{|l|}{ AUG } \\
\hline $27 \ldots$ & - - & - - & - - & - - & - - & - - & - - & - - & - - & - - \\
\hline $27 \ldots$ & 1.9 & 11 & 254 & 44.3 & 34.8 & 10.5 & 1.55 & 213 & 21.5 & 25.9 \\
\hline 27. & 1.5 & 12 & 253 & 45.3 & 33.9 & 9.80 & 1.53 & 216 & 21.6 & 25.0 \\
\hline $27 \ldots$ & 1.4 & 9 & 317 & 67.6 & 36.0 & 10.4 & 1.82 & 270 & 23.6 & 28.8 \\
\hline $27 \ldots$ & 4.4 & 11 & 331 & 71.5 & 37.1 & 10.9 & 1.89 & 288 & 24.4 & 28.8 \\
\hline $27 \ldots$ & 9.4 & 9 & 330 & 71.2 & 37.0 & 10.5 & 1.93 & 296 & 27.3 & 28.3 \\
\hline
\end{tabular}


424840088241600 LAKE BEULAH AT DEEP HOLE NEAR EAST TROY, WI

LAKE-DEPTH PROFILES, MAY 13 TO AUGUST 27, 2014

05-13-14

08-27-14

DISSOLVED OXYGEN (D.O.), IN MILLIGRAMS PER LITER

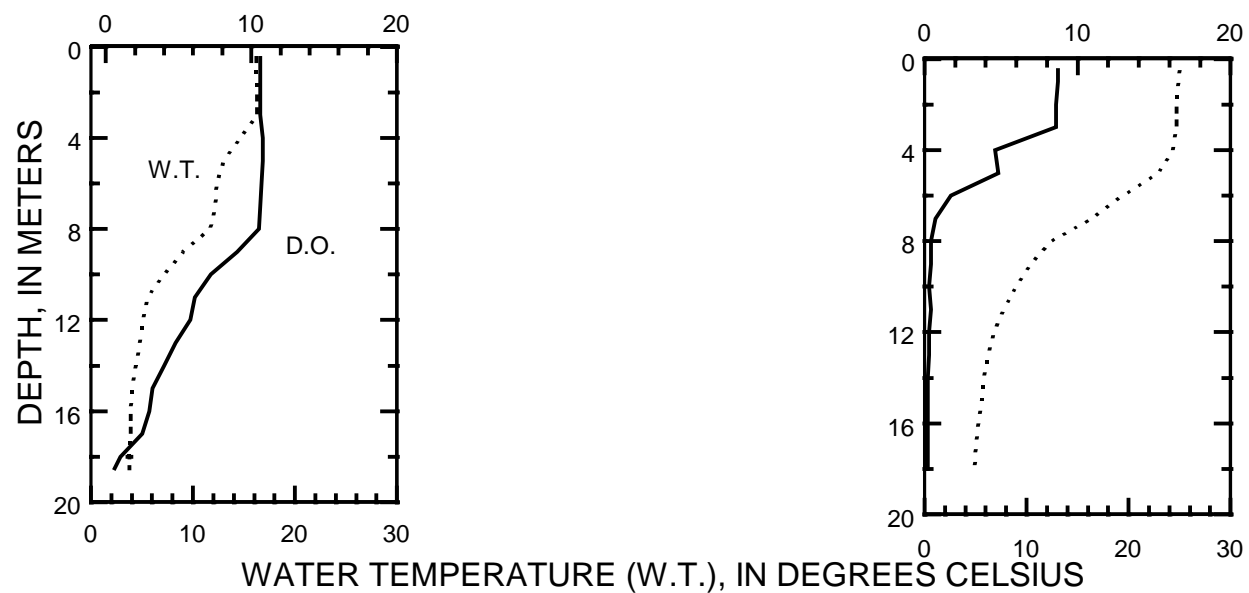

PH, IN STANDARD UNITS
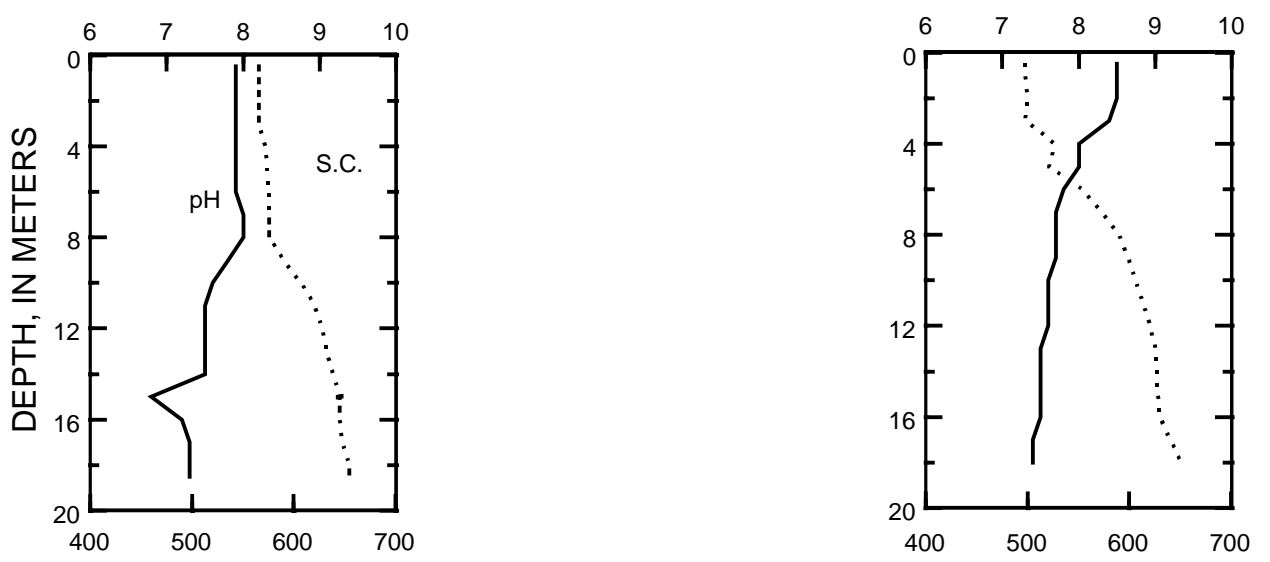

SPECIFIC CONDUCTANCE (S.C.), IN MICROSIEMENS PER CENTIMETER AT 25 DEGREES CELSIUS 

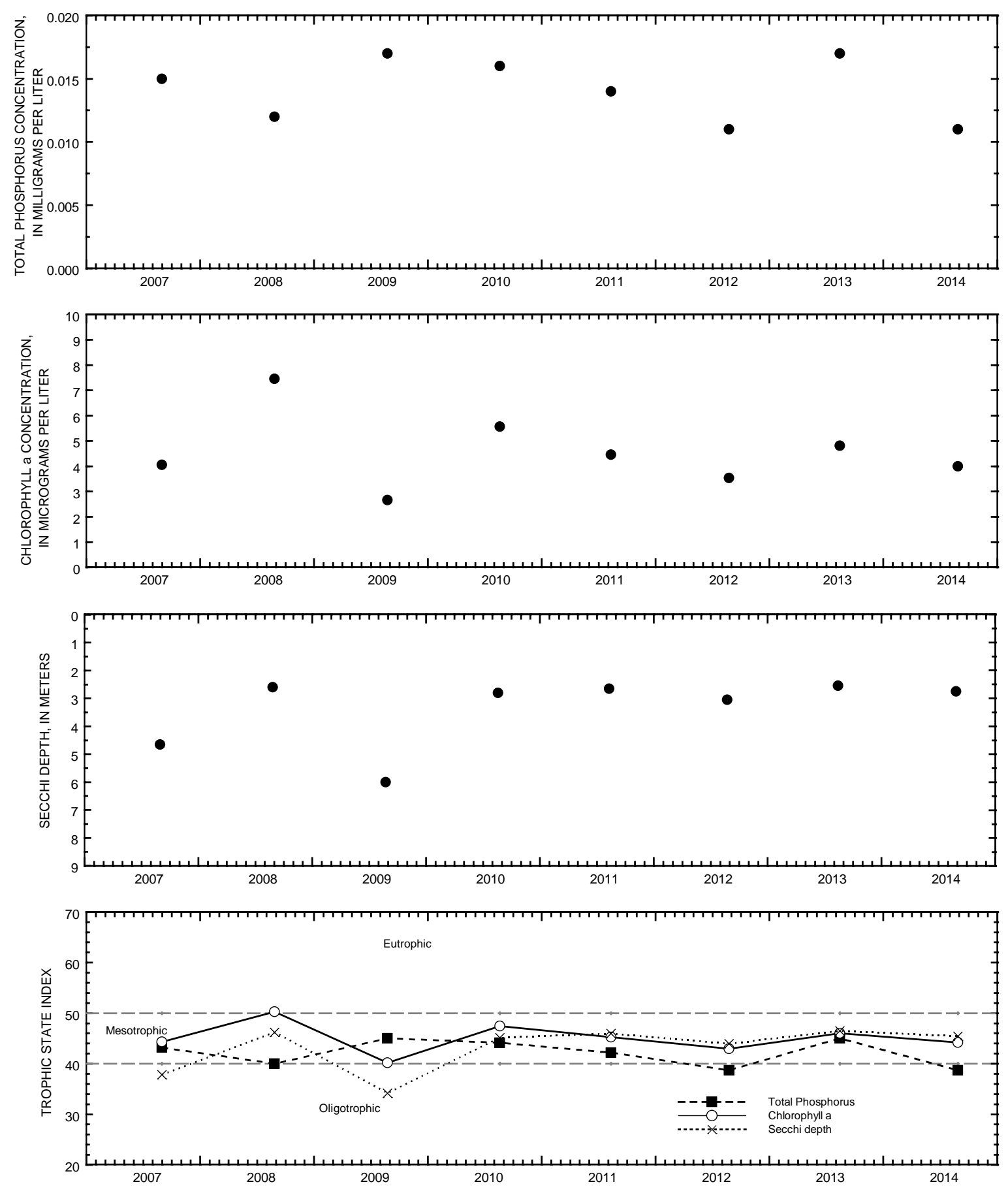

August surface total phosphorus, chlorophyll a concentrations, Secchi depths, and TSI data for Lake Beulah, Deep Hole, near East Troy, Wisconsin. 
424929088231300 LAKE BEULAH STATION 2 NEAR EAST TROY, WI

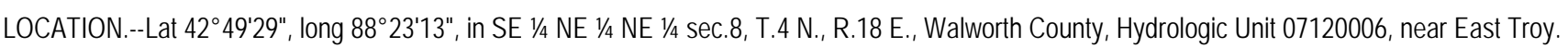

SURFACE AREA.--1.30 mi².

PERIOD OF RECORD.--August 2007 to August 2014.

REMARKS.--Lake sampled at a depth of $15 \mathrm{~m}$. Water-quality analyses by Wisconsin State Laboratory of Hygiene.

WATER-QUALITY DATA, MAY 13 TO AUGUST 27, 2014

(Milligrams per liter unless otherwise indicated)

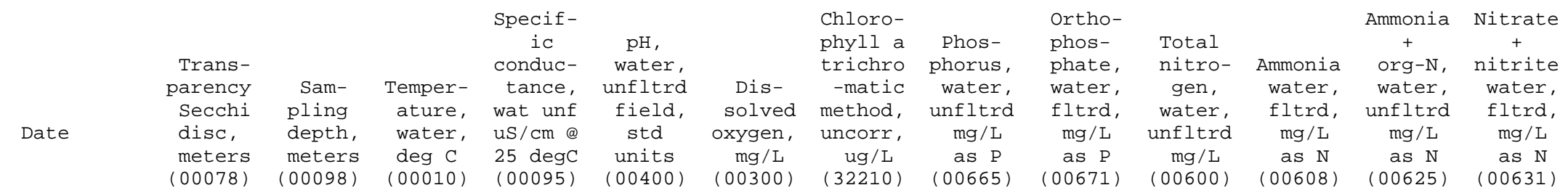

MAY 2014

\begin{tabular}{|c|c|c|c|c|c|c|c|c|c|c|c|c|c|}
\hline $13 \ldots$ & 5.25 & - - & - - & -- & -- & - - & -- & - & - - & - - & - - & - - & - - \\
\hline $13 \ldots$ & - - & 0.50 & 17.0 & 494 & 8.4 & 11.0 & 2.20 & 0.011 & $\odot .006$ & 0.67 & 0.010 & 0.54 & 0.130 \\
\hline \multicolumn{14}{|l|}{ AUG } \\
\hline $27 \ldots$ & 3.75 & - - & - - & - - & - - & - - & - - & - - & - - & - - & - - & - - & - - \\
\hline $27 \ldots$ & - - & 2.0 & 25.0 & 448 & 8.4 & 8.4 & 4.00 & $\odot . \odot \odot 8$ & $\odot .003$ & $<0.60$ & 0.010 & 0.58 & $<0.020$ \\
\hline $27 \ldots$ & - - & 4.0 & 24.8 & 446 & 8.4 & 8.2 & - - & 0.012 & - - & -- & - & -- & -- \\
\hline $27 \ldots$ & - - & 12.0 & 6.6 & 590 & 7.5 & 0.7 & - - & 0.013 & - - & - - & - - & - - & - - \\
\hline $27 \ldots$ & - & 14.0 & 5.4 & $6 \odot 4$ & 7.4 & 0.4 & - & $\odot .042$ & - - & - & -- & -- & - \\
\hline $27 \ldots$ & -- & 15.0 & 5.1 & $6 \odot 8$ & 7.4 & 0.3 & - & $\odot .041$ & $\odot . \odot \odot 3$ & $<1.6$ & 0.820 & 1.6 & $<0.020$ \\
\hline
\end{tabular}


424929088231300 LAKE BEULAH STATION 2 NEAR EAST TROY, WI

WATER-QUALITY DATA, MAY 13 TO AUGUST 27, 2014

(Milligrams per liter unless otherwise indicated)

Turbdty Appar -

white ent

light, color, Hard-

det ang water, ness,

water,

degrees Pt-Co

NTU

units $\mathrm{CaCO} 3$

(63675) (00081)

$(\odot$ COC03

Calcium
water,
fltrd,
mg/L
$(00915)$

Magnes-

Magnes -

ium, Sodium,

water, water,

fltrd,

$\mathrm{mg} / \mathrm{L}$

fltrd,

$\mathrm{mg} / \mathrm{L}$
$(\odot \odot 930)$

ANC,

sium, end

Chlor

Sulfate

fltrd water

MAY 2014

$13 \ldots$

$\begin{array}{rcr}-- & -- & -- \\ 0.7 & 11 & 243 \\ -- & -- & -- \\ 1.6 & 8 & 211 \\ -- & -- & -- \\ -- & -- & -- \\ -- & -- & -- \\ 13 & 12 & 302\end{array}$

--
45.0
--
31.4
--
--
--
60.0

--
31.8
--
32.1
--
--
--
37.0

--
11.0
10.8
--
--
--
11.4

$\begin{array}{lr}-\overline{-} & -\overline{7} \\ 1.73 & 208\end{array}$

$(00940)$

fltrd

13.

$27 .$.

$27 \ldots$

$27 \ldots$

$27 \ldots$

13

- - -

25.5

24.5

$1.63 \quad 18$

24

- -

1.98

--
--
268

27.7

-

- - 
424929088231300 LAKE BEULAH STATION 2 NEAR EAST TROY, WI

LAKE-DEPTH PROFILES, MAY 13 TO AUGUST 27, 2014

05-13-14

08-27-14

DISSOLVED OXYGEN (D.O.), IN MILLIGRAMS PER LITER

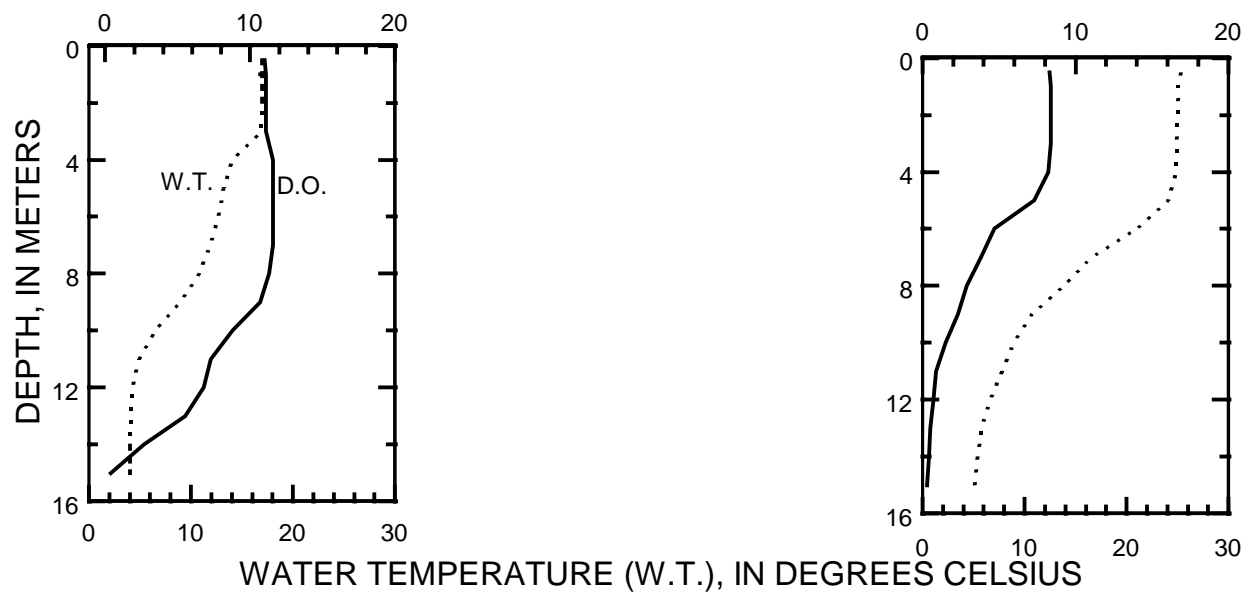

$\mathrm{PH}$, IN STANDARD UNITS
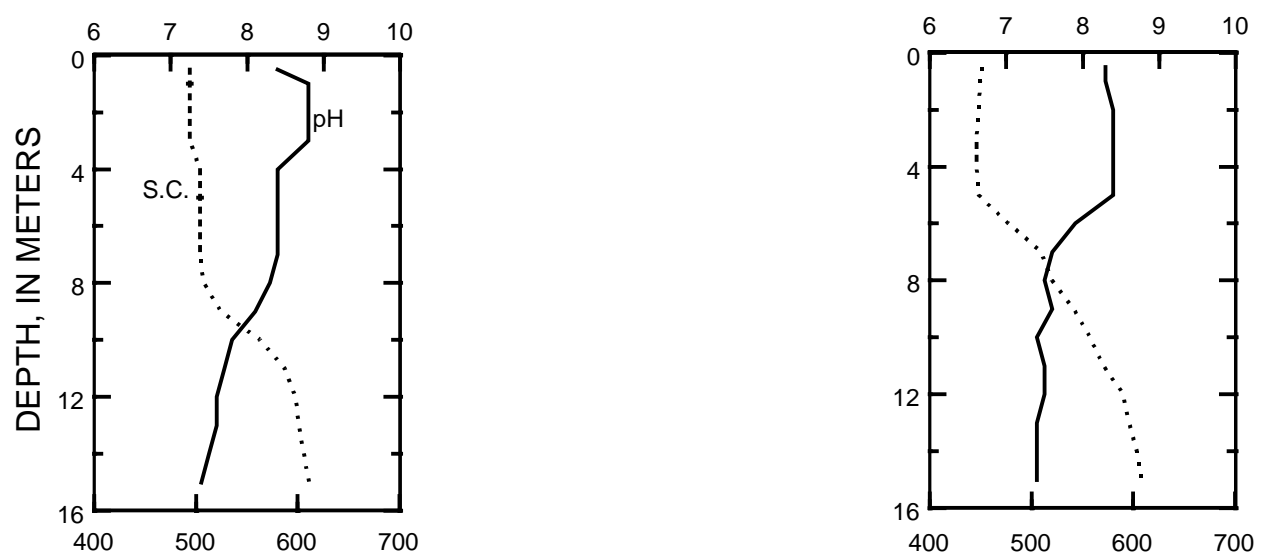

SPECIFIC CONDUCTANCE (S.C.), IN MICROSIEMENS PER CENTIMETER AT 25 DEGREES CELSIUS 

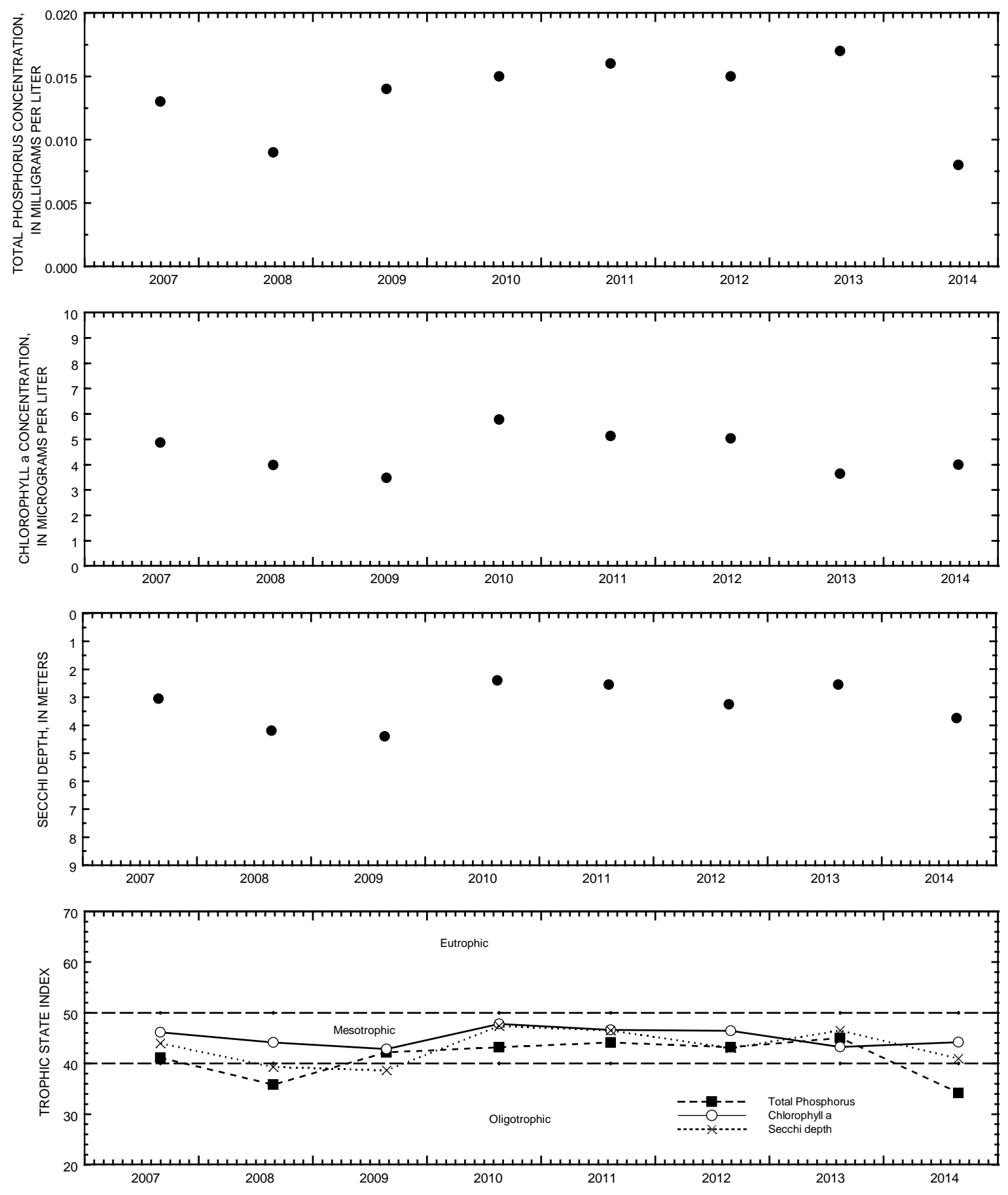

August surface total phosphorus, chlorophyll a concentrations, Secchi depths, and TSI data for Lake Beulah, Station 2, near East Troy, Wisconsin. 


\section{LAKE BEULAH INLET NEAR EAST TROY, WI}

LOCATION.--Lat 4248'39", long 88²4'56", in SE 1/4 NE 1/4 NW 1/4 sec.18, T.4 N., R.18 E., Walworth County, Hydrologic Unit 07120006, near East Troy

PERIOD OF RECORD.--August 2008 to August 2014.

REMARKS.--Lake sampled at a depth of $15 \mathrm{~m}$. Water-quality analyses by Wisconsin State Laboratory of Hygiene.

WATER-QUALITY DATA, MAY 13 TO AUGUST 27, 2014

(Milligrams per liter unless otherwise indicated)

\begin{tabular}{|c|c|c|c|c|c|c|c|c|c|c|c|c|c|}
\hline Date & $\begin{array}{c}\text { Trans- } \\
\text { parency } \\
\text { Secchi } \\
\text { disc, } \\
\text { meters } \\
(00078)\end{array}$ & $\begin{array}{c}\text { Sam- } \\
\text { pling } \\
\text { depth, } \\
\text { meters } \\
(\odot \odot \odot 98)\end{array}$ & $\begin{array}{c}\text { Temper - } \\
\text { ature, } \\
\text { water, } \\
\text { deg C } \\
(00010)\end{array}$ & $\begin{array}{c}\text { Specif- } \\
\text { ic } \\
\text { conduc- } \\
\text { tance, } \\
\text { wat unf } \\
\text { uS/cm @ } \\
25 \mathrm{degC} \\
(\odot \odot \odot 95)\end{array}$ & $\begin{array}{c}\text { pH, } \\
\text { water, } \\
\text { unfltrd } \\
\text { field, } \\
\text { std } \\
\text { units } \\
(\odot \odot 4 \odot \odot)\end{array}$ & $\begin{array}{c}\text { Dis- } \\
\text { solved } \\
\text { oxygen, } \\
\text { mg/L } \\
(\odot \odot 3 \odot \odot)\end{array}$ & $\begin{array}{c}\text { Chloro- } \\
\text { phyll a } \\
\text { trichro } \\
\text {-matic } \\
\text { method, } \\
\text { uncorr, } \\
\text { ug/L } \\
(32210)\end{array}$ & $\begin{array}{c}\text { Phos- } \\
\text { phorus, } \\
\text { water, } \\
\text { unfltrd } \\
\text { mg/L } \\
\text { as } P \\
(00665)\end{array}$ & $\begin{array}{c}\text { Ortho- } \\
\text { phos- } \\
\text { phate, } \\
\text { water, } \\
\text { fltrd, } \\
\text { mg/L } \\
\text { as P } \\
(00671)\end{array}$ & $\begin{array}{c}\text { Total } \\
\text { nitro- } \\
\text { gen, } \\
\text { water, } \\
\text { unfltrd } \\
\text { mg/L } \\
(\odot \odot 6 \odot \odot)\end{array}$ & $\begin{array}{c}\text { Ammonia } \\
\text { water, } \\
\text { fltrd, } \\
\text { mg/L } \\
\text { as N } \\
(0 \odot 608)\end{array}$ & $\begin{array}{c}\text { Ammonia } \\
+ \\
\text { org-N, } \\
\text { water, } \\
\text { unfltrd } \\
\text { mg/L } \\
\text { as N } \\
(00625)\end{array}$ & $\begin{array}{c}\text { Nitrate } \\
+ \\
\text { nitrite } \\
\text { water, } \\
\text { fltrd, } \\
\text { mg/L } \\
\text { as N } \\
(00631)\end{array}$ \\
\hline
\end{tabular}

MAY 2014

$13 \ldots$

$\begin{array}{ccrrr}1.00 & -- & -- & -- & -- \\ -- & 0.50 & 16.7 & 515 & 7.7 \\ >1.00 & -- & -- & -- & -- \\ -- & 0.50 & 22.2 & 611 & 7.9\end{array}$

5.3

3.10

๑. 025

$\odot .008$

1.4

$\odot .070$

$\odot .64$

$\odot .710$

AUG

$27 \ldots$

0.50

- -

$--$

$--$

$\odot . \odot-$

0.57

0.050

0.42

150

Turbdty Appar-

white ent

light, color

Hard-

det ang

$90+/-30$ unfltri

degrees Pt-Co

ness,

Magnes-

ANC,

Date

$\begin{array}{cc}\text { NTU } & \text { units } \\ (63675) & (00081)\end{array}$

$\mathrm{mg} / \mathrm{L}$ as

water, water, Sodium,

water, water, water, lab,

fltrd, fltrd,

fltrd, $\mathrm{mg} / \mathrm{L}$ a

$\mathrm{mg} / \mathrm{L}$

$\mathrm{mg} / \mathrm{L}$

$\mathrm{mg} / \mathrm{L}$

$\mathrm{CaC03}$
$(\odot \odot 417)$

Chlor -

ide, sulfate

water, water

( 00900$)$

(00915)

$(\odot \odot 925)$

MAY 2014

$13 \ldots$

AUG

$27 \ldots$

$\begin{array}{rcr}-- & -- & -- \\ 1.8 & 35 & 268 \\ -- & -- & -- \\ <0.1 & 26 & 309\end{array}$

--
60.9
--
63.0

28.1

7.30

2.10

232

$\mathrm{mg} / \mathrm{L}$

fltrd,

(००940) (००945)

$27 \ldots$

$<0.1$

63.

9.20

1.78

--
276

17.6

19.3

22.1

24.6 


\section{BIG CEDAR LAKE, NORTH SITE, NEAR WEST BEND, WI}

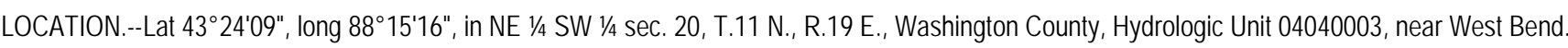
SURFACE AREA.--1.46 mi².

PERIOD OF RECORD.--February 2000 to current year.

REMARKS.--Lake sampled on north side at a depth of $12 \mathrm{~m}$. Water-quality analyses by Wisconsin State Laboratory of Hygiene.

WATER-QUALITY DATA, MARCH 5 TO AUGUST 20, 2014

(Milligrams per liter unless otherwise indicated)

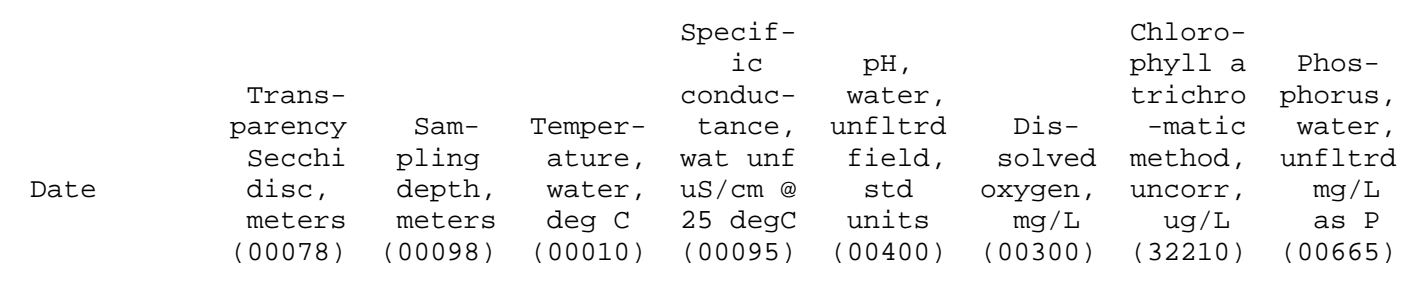

\begin{tabular}{|c|c|c|c|c|c|c|c|c|}
\hline \multicolumn{9}{|c|}{ MAR 2014} \\
\hline $05 \ldots$ & - - & 1.0 & 2.7 & 654 & 8.1 & 9.3 & 1.06 & 0.012 \\
\hline $05 \ldots$ & -- & 11.0 & 3.6 & 828 & 7.6 & 1.8 & - - & 0.018 \\
\hline \multicolumn{9}{|l|}{ MAY } \\
\hline $21 \ldots$ & 7.65 & -- & - - & - - & - - & - & -- & - - \\
\hline $21 \ldots$ & -- & 0.50 & 16.9 & 578 & 8.3 & 11.7 & 0.872 & 0.009 \\
\hline \multicolumn{9}{|l|}{ JUN } \\
\hline $30 \ldots$ & 5.55 & - & -- & -- & -- & -- & -- & - - \\
\hline $30 \ldots$ & -- & 0.50 & 24.7 & 540 & 8.7 & 8.7 & 4.96 & 0.016 \\
\hline $30 \ldots$ & - & 10.5 & 13.7 & 590 & 7.7 & 0.4 & -- & 0.026 \\
\hline \multicolumn{9}{|l|}{ JUL } \\
\hline 29 & 2.30 & -- & - - & -- & - - & -- & -- & - - \\
\hline $29 \ldots$ & -- & 0.50 & 23.4 & 525 & 8.6 & 8.8 & 5.33 & 0.018 \\
\hline $29 \ldots$ & -- & 10.5 & 14.3 & 582 & 7.4 & 0.3 & - - & 0.033 \\
\hline \multicolumn{9}{|l|}{ AUG } \\
\hline $20 \ldots$ & 3.25 & - - & - - & -- & -- & -- & -- & - - \\
\hline $20 \ldots$ & - - & 0.50 & 24.5 & 542 & 8.5 & 8.6 & 3.74 & 0.015 \\
\hline $20 \ldots$ & - - & 12.5 & 13.4 & 627 & 7.5 & 0.3 & - - & 0.043 \\
\hline
\end{tabular}


432409088151600 BIG CEDAR LAKE, NORTH SITE, NEAR WEST BEND, WI

LAKE-DEPTH PROFILES, MARCH 5 TO AUGUST 20, 2014

03-05-14

05-21-14

06-30-14

$07-29-14$

08-20-14

DISSOLVED OXYGEN (D.O.), IN MILLIGRAMS PER LITER
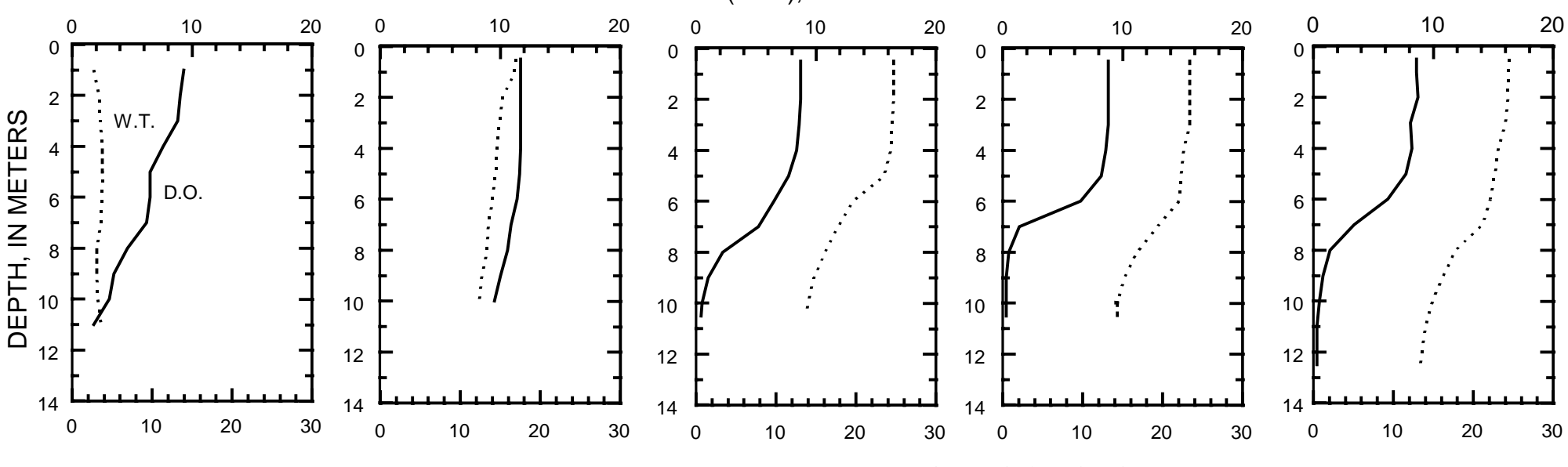

WATER TEMPERATURE (W.T.), IN DEGREES CELSIUS
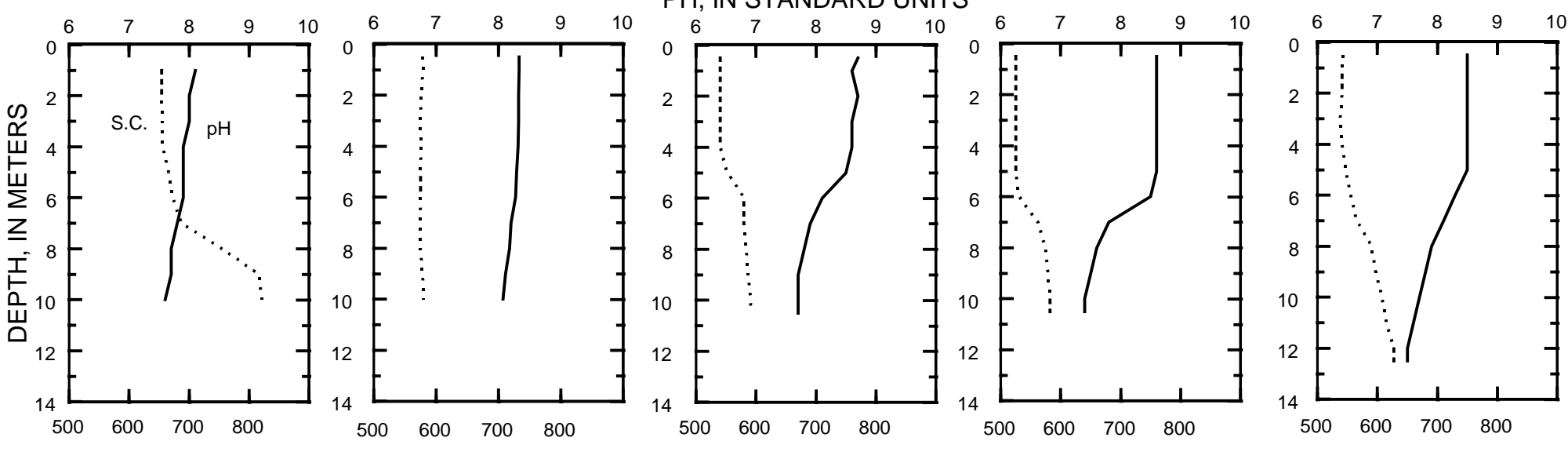

SPECIFIC CONDUCTANCE (S.C.), IN MICROSIEMENS PER CENTIMETER AT 25 DEGREES CELSIUS 

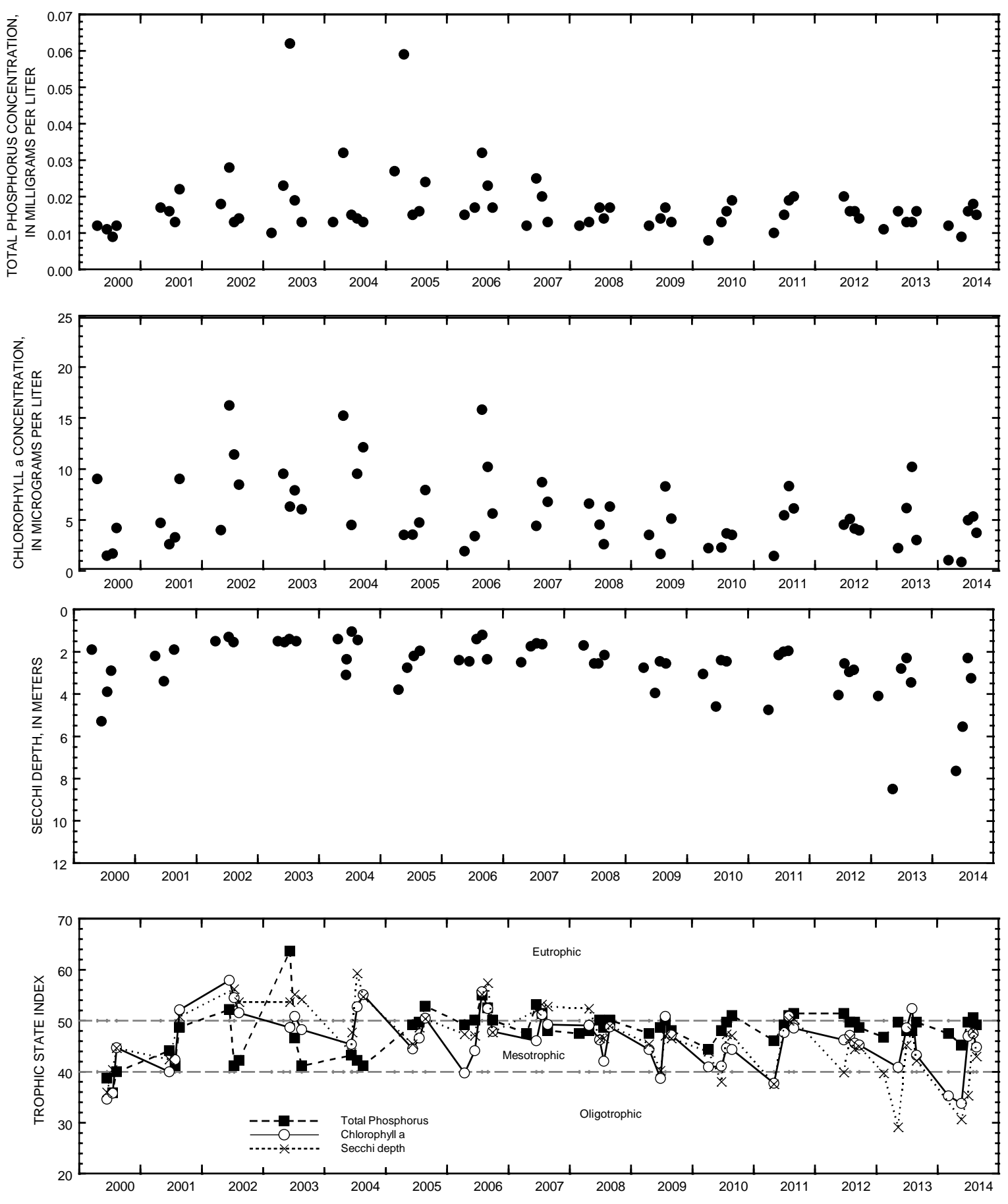

Surface total phosphorus, chlorophyll a concentrations, Secchi depths, and TSI data for Big Cedar Lake, North Site, near West Bend, Wisconsin. 
432224088154900 BIG CEDAR LAKE, SOUTH SITE, NEAR WEST BEND, WI

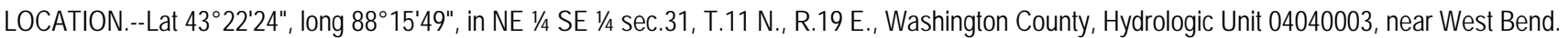
SURFACE AREA.--1.46 mi².

PERIOD OF RECORD.--February 2000 to current year.

REMARKS.--Lake sampled on south side at deep hole. Water-quality analyses by Wisconsin State Laboratory of Hygiene.

WATER-QUALITY DATA, MARCH 5 TO AUGUST 20, 2014

(Milligrams per liter unless otherwise indicated)

\begin{tabular}{|c|c|c|c|c|c|c|c|c|c|c|c|c|c|}
\hline Date & $\begin{array}{c}\text { Trans- } \\
\text { parency } \\
\text { Secchi } \\
\text { disc, } \\
\text { meters } \\
(\odot \odot \odot 78)\end{array}$ & $\begin{array}{c}\text { Sam- } \\
\text { pling } \\
\text { depth, } \\
\text { meters } \\
(\odot \odot 098)\end{array}$ & $\begin{array}{c}\text { Temper - } \\
\text { ature, } \\
\text { water, } \\
\text { deg C } \\
(00010)\end{array}$ & $\begin{array}{c}\text { Specif- } \\
\text { ic } \\
\text { conduc- } \\
\text { tance, } \\
\text { wat unf } \\
\text { uS/cm @ } \\
25 \text { degc } \\
(00095)\end{array}$ & $\begin{array}{c}\text { pH, } \\
\text { water, } \\
\text { unfltrd } \\
\text { field, } \\
\text { std } \\
\text { units } \\
(00400)\end{array}$ & $\begin{array}{c}\text { Dis- } \\
\text { solved } \\
\text { oxygen, } \\
\text { mg/L } \\
(00300)\end{array}$ & $\begin{array}{l}\text { Chloro- } \\
\text { phyll a } \\
\text { trichro } \\
\text {-matic } \\
\text { method, } \\
\text { uncorr, } \\
\text { ug/L } \\
(32210)\end{array}$ & $\begin{array}{c}\text { Phos- } \\
\text { phorus, } \\
\text { water, } \\
\text { unfltrd } \\
\mathrm{mg} / \mathrm{L} \\
\text { as } \mathrm{P} \\
(00665)\end{array}$ & $\begin{array}{l}\text { Ortho- } \\
\text { phos- } \\
\text { phate, } \\
\text { water, } \\
\text { fltrd, } \\
\text { mg/L } \\
\text { as P } \\
(00671)\end{array}$ & $\begin{array}{c}\text { Total } \\
\text { nitro- } \\
\text { gen, } \\
\text { water, } \\
\text { unfltrd } \\
\mathrm{mg} / \mathrm{L} \\
(00600)\end{array}$ & $\begin{array}{c}\text { Ammonia } \\
\text { water, } \\
\text { fltrd, } \\
\text { mg/L } \\
\text { as N } \\
(00608)\end{array}$ & $\begin{array}{c}\text { Ammonia } \\
+ \\
\text { org-N, } \\
\text { water, } \\
\text { fltrd, } \\
\text { mg/L } \\
\text { as N } \\
(00623)\end{array}$ & $\begin{array}{c}\text { Ammonia } \\
+ \\
\text { org-N, } \\
\text { water, } \\
\text { unfltrd } \\
\text { mg/L } \\
\text { as N } \\
(00625)\end{array}$ \\
\hline \multicolumn{14}{|l|}{ MAR 2014} \\
\hline $05 \ldots$ & -- & 1.0 & 1.6 & 584 & 8.1 & 10.9 & 1.20 & 0.011 & -- & - - & - - & - - & -- \\
\hline \multicolumn{14}{|l|}{ MAY } \\
\hline $21 \ldots$ & 7.85 & -- & -- & -- & -- & -- & - - & - - & - - & - - & -- & - - & -- \\
\hline $21 \ldots$ & - - & 0.50 & 15.3 & 568 & 8.4 & 13.3 & 0.276 & 0.007 & 0.002 & 0.64 & 0.031 & -- & 0.41 \\
\hline \multicolumn{14}{|l|}{ JUN } \\
\hline $30 \ldots$ & 4.75 & -- & -- & -- & -- & -- & -- & -- & -- & -- & -- & -- & -- \\
\hline $30 \ldots$ & -- & 0.50 & 23.3 & 555 & 8.5 & 8.9 & 0.935 & 0.010 & - - & -- & -- & -- & - - \\
\hline $30 \ldots$ & -- & 30.5 & 5.4 & 592 & 7.6 & 0.9 & -- & 0.078 & -- & -- & -- & -- & -- \\
\hline \multicolumn{14}{|l|}{ JUL } \\
\hline $29 \ldots$ & 3.00 & - & -- & -- & - & -- & -- & - & - & - & - & -- & -- \\
\hline $29 \ldots$ & -- & 0.50 & 23.1 & 533 & 8.8 & 9.7 & 5.02 & 0.013 & $\odot .003$ & 0.44 & $<0.015$ & 0.61 & 0.40 \\
\hline $20 \ldots$ & 4.35 & -- & -- & -- & -- & -- & -- & -- & -- & -- & -- & -- & -- \\
\hline $20 \ldots$ & -- & 0.50 & 24.0 & 531 & 8.8 & 9.3 & 2.82 & 0.012 & -- & -- & -- & -- & -- \\
\hline $20 \ldots$ & - - & 30.5 & 5.5 & 610 & 7.6 & 0.8 & -- & $\odot .019$ & -- & -- & -- & -- & - - \\
\hline
\end{tabular}


432224088154900 BIG CEDAR LAKE, SOUTH SITE, NEAR WEST BEND, WI

WATER-QUALITY DATA, MARCH 5 TO AUGUST 20, 2014

(Milligrams per liter unless otherwise indicated)

\begin{tabular}{|c|c|c|c|c|c|c|c|c|c|c|c|c|c|}
\hline & Nitrate & Turbdty & Appar - & & & & & & ANC, & & & & \\
\hline ate & $\begin{array}{c}\text { nitrite } \\
\text { water, } \\
\text { fltrd, } \\
\text { mg/L } \\
\text { as N } \\
(00631)\end{array}$ & $\begin{array}{c}\text { light, } \\
\text { det ang } \\
90+/-30 \\
\text { degrees } \\
\text { NTU } \\
(63675)\end{array}$ & $\begin{array}{c}\text { color, } \\
\text { water, } \\
\text { unfltrd } \\
\text { Pt-Co } \\
\text { units } \\
(00 \odot 81)\end{array}$ & $\begin{array}{l}\text { Hard- } \\
\text { ness, } \\
\text { water, } \\
\text { mg/L as } \\
\text { CaC03 } \\
(0 \odot 9 \odot \odot)\end{array}$ & $\begin{array}{c}\text { Calcium } \\
\text { water, } \\
\text { fltrd, } \\
\text { mg/L } \\
(00915)\end{array}$ & $\begin{array}{c}\text { Magnes- } \\
\text { ium, } \\
\text { water, } \\
\text { fltrd, } \\
\text { mg/L } \\
(00925)\end{array}$ & $\begin{array}{c}\text { Sodium, } \\
\text { water, } \\
\text { fltrd, } \\
\text { mg/L } \\
(00930)\end{array}$ & $\begin{array}{l}\text { Potas- } \\
\text { sium, } \\
\text { water, } \\
\text { fltrd, } \\
\text { mg/L } \\
(00935)\end{array}$ & $\begin{array}{c}\text { fixed } \\
\text { end pt, } \\
\text { lab, } \\
\mathrm{mg} / \mathrm{L} \text { as } \\
\text { CaC03 } \\
(00417)\end{array}$ & $\begin{array}{c}\text { Chlor - } \\
\text { ide, } \\
\text { water, } \\
\text { fltrd, } \\
\text { mg/L } \\
(00940)\end{array}$ & $\begin{array}{c}\text { Sulfate } \\
\text { water, } \\
\text { fltrd, } \\
\text { mg/L } \\
(00945)\end{array}$ & $\begin{array}{c}\text { Silica, } \\
\text { water, } \\
\text { fltrd, } \\
\text { mg/L as } \\
\text { Si02 } \\
(00955)\end{array}$ & $\begin{array}{c}\text { Iron, } \\
\text { water, } \\
\text { fltrd, } \\
\text { ug/L } \\
(01046)\end{array}$ \\
\hline
\end{tabular}

MAR 2014

05....

MAY

$21 \ldots$

JUN

$30 .$.

$30 \ldots$

JUL

$29 \ldots$

$29 \ldots$

AUG

$20 \ldots$

$20 \ldots$

$20 \ldots$

$\begin{array}{cc}--- & -- \\ 0.236 & 0.5 \\ -- & -- \\ -- & -- \\ -- & -- \\ -- & -- \\ 0.035 & -- \\ -- & -- \\ -- & -- \\ -- & -- \\ -- & -- \\ & \\ & \\ & \begin{array}{r}\text { Dis- } \\ \text { solved } \\ \text { solids }\end{array} \\ \text { Manga- } & \begin{array}{c}\text { dried @ } \\ \text { nese, }\end{array} \\ \text { water, } & \text { 180degC } \\ \text { fltrd, } & \text { wat flt } \\ \text { ug/L } & \text { mg/L } \\ \text { (01056) } & \text { (70300) }\end{array}$

MAR 2014

$05 \ldots$

AY

21...

$-$

(0900)

$(\odot \odot 925) \quad(\odot \odot 930)$

$10^{-} \quad 238$

$--$

37.4

35.1

25.0

$\begin{array}{ll}-- & -- \\ 1.68 & 209\end{array}$

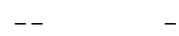

$--$

- - -

- -

- -

209

56

$20 . \overline{6}$

3.66

$<0.100$

$$
\text { - }
$$$$
-
$$$$
--
$$$$
\text { - - }
$$$$
-
$$

is -

solids

Date

$$
\begin{array}{cc}
\mathrm{ug} / \mathrm{L} & \mathrm{mg} / \mathrm{L} \\
(01056) & (70300)
\end{array}
$$

$21 \ldots$

$<1 . \odot \odot$ 


\section{BIG CEDAR LAKE, SOUTH SITE, NEAR WEST BEND, WI}

LAKE-DEPTH PROFILES, MARCH 5 TO AUGUST 20, 2014

03-05-14

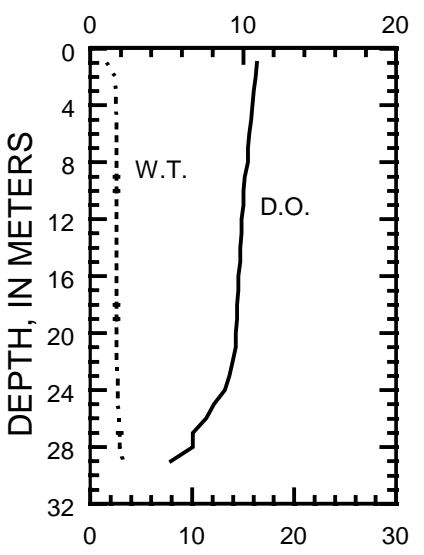

05-21-14

DSOLVED OXYGEN (D.O.), IN MILLIGRAMS PER LITER
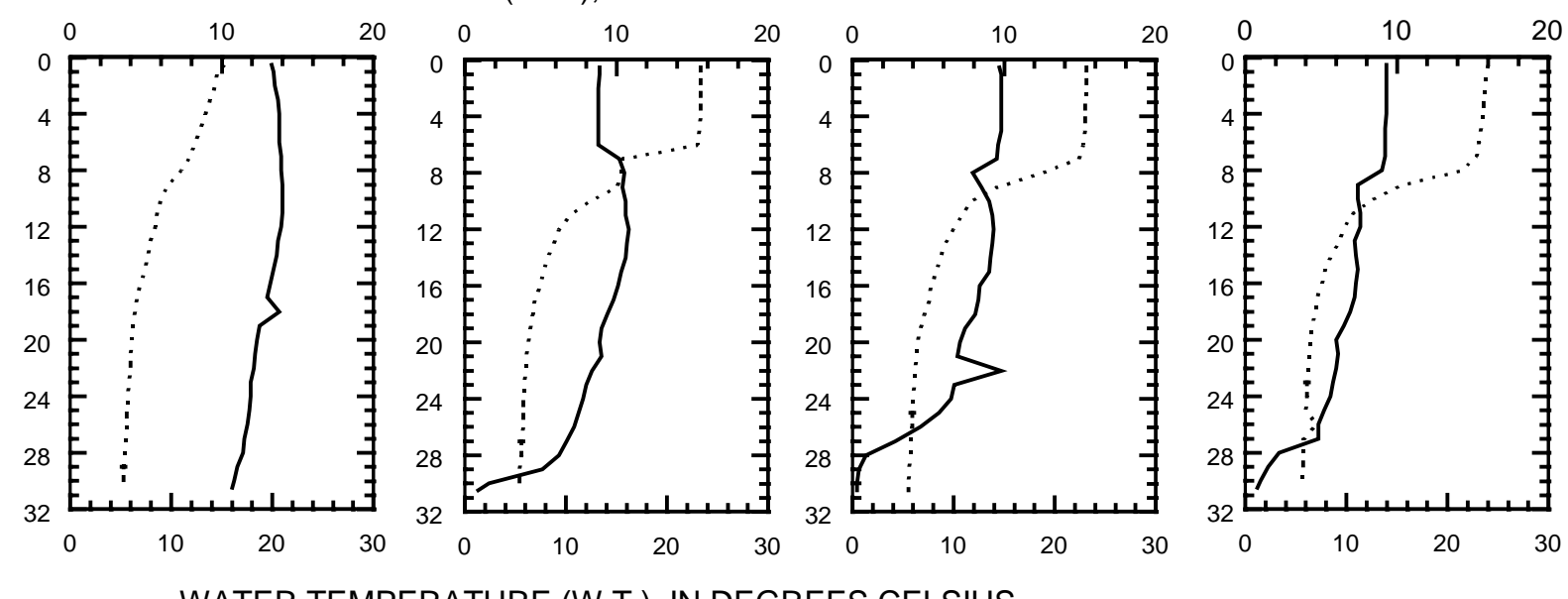

PH, IN STANDARD UNITS
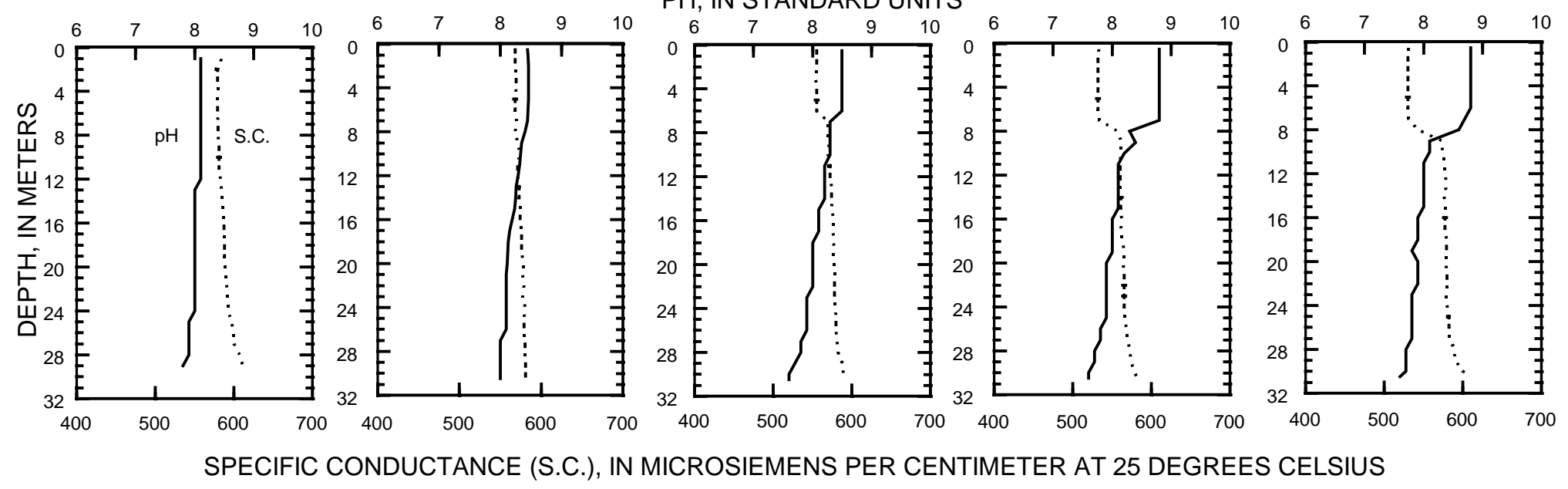

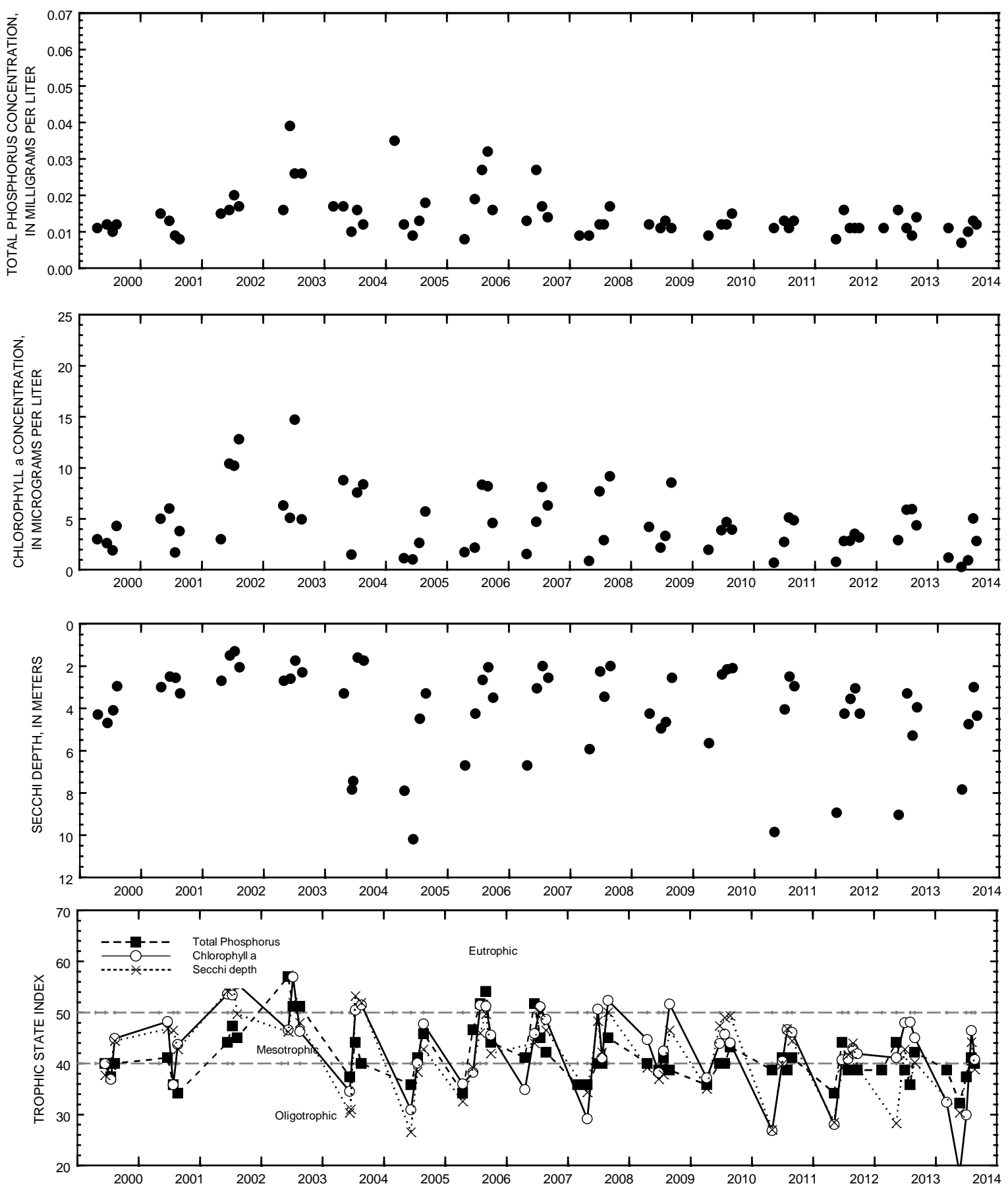

Surface total phosphorus, chlorophyll a concentrations, Secchi depths, and TSI data for Big Cedar Lake, South Site, near West Bend, Wisconsin. 


\section{DELAVAN LAKE AT CENTER NEAR DELAVAN LAKE, W}

LOCATION.--Lat $42^{\circ} 35^{\prime} 56^{\prime \prime}$, long $88^{\circ} 36^{\prime} 50^{\prime \prime}$, in SE 1/4 SW 1/4 Sec.28, T.2 N., R.16 E., Walworth County, Hydrologic Unit 07090001, 2.6 mi southeast of Delavan

SURFACE AREA.--3.24 mi².

DRAINAGE AREA.--41.4 mi², of which $2.3 \mathrm{mi}^{2}$ is non-contributing.

PERIOD OF RECORD.--October 1983 to September 2009, October 2011 to September 2014

REMARKS.-- Water-quality analyses by Wisconsin State Laboratory of Hygiene.

WATER-QUALITY DATA, NOVEMBER 19, 2013 TO JULY 15, 2014

(Milligrams per liter unless otherwise indicated)

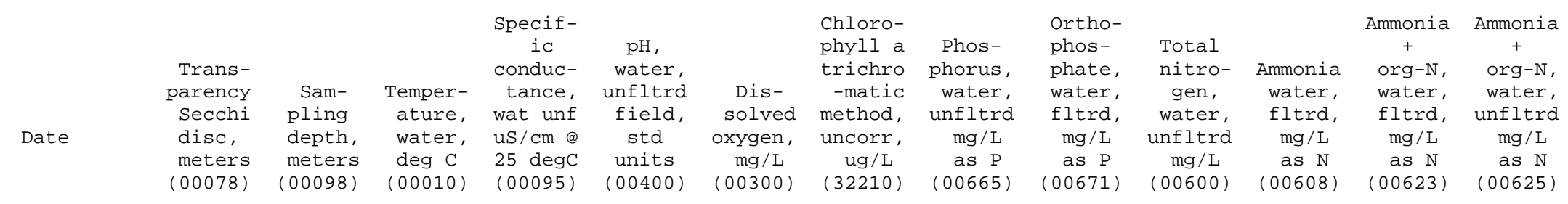

\begin{tabular}{|c|c|c|c|c|c|c|c|c|c|c|c|c|c|}
\hline \multicolumn{14}{|l|}{ NOV 2013} \\
\hline $19 \ldots$ & 4.85 & - - & - - & - - & - - & - - & - - & - - & - - & - - & - - & - & - - \\
\hline $19 \ldots$ & - - & 0.50 & 7.1 & 544 & 7.6 & 10.7 & 8.66 & 0.039 & 0.024 & $\odot .73$ & 0.128 & 0.68 & 0.61 \\
\hline \multicolumn{14}{|l|}{ MAR 2014} \\
\hline $04 \ldots$ & 10.2 & - - & - - & - - & - & - - & - - & - - & - - & - - & - - & - - & - - \\
\hline $04 \ldots$ & -- & 1.0 & 1.1 & 605 & 8.0 & 11.9 & 1.40 & 0.034 & $\odot .025$ & 0.80 & 0.150 & 0.76 & 0.56 \\
\hline$\odot 4 \ldots$ & -- & 16.0 & 4.3 & 1280 & 7.5 & 1.3 & - - & $\odot .086$ & $\odot .069$ & - - & -- & - - & - - \\
\hline \multicolumn{14}{|l|}{ MAY } \\
\hline $15 \ldots$ & $8.4 \odot$ & - - & - - & - - & - - & - - & - - & - - & - - & - - & - - & - - & - - \\
\hline $15 \ldots$ & -- & 0.50 & 13.5 & 562 & 8.3 & 12.4 & 1.30 & 0.026 & 0.013 & $\odot .72$ & 0.111 & - & 0.62 \\
\hline $15 \ldots$ & -- & 16.0 & 10.8 & 571 & 7.9 & 6.7 & -- & 0.077 & 0.053 & -- & -- & -- & - - \\
\hline $27 \ldots$ & 3.35 & - - & - - & - - & - - & - - & - - & - - & - - & - - & - - & - - & - - \\
\hline $27 \ldots$ & -- & 0.50 & 24.3 & 9 & 570 & 11.4 & 11.7 & 0.023 & $<0.0 \odot 2$ & $<0.62$ & $<0.015$ & 0.61 & 0.60 \\
\hline $27 \ldots$ & - - & 7.0 & 20.5 & 8 & 584 & 5.7 & - & 0.018 & $\odot . \odot \odot 2$ & - & - & - & - \\
\hline $27 \ldots$ & -- & 10.0 & 13.6 & 8 & 593 & 1.0 & -- & $\odot .098$ & $\odot .076$ & - & - & - & - \\
\hline $27 \ldots$ & - - & 16.0 & 11.5 & 8 & 610 & 0.1 & - - & 0.381 & $\odot .341$ & -- & - & - & - \\
\hline \multicolumn{14}{|l|}{ JUL } \\
\hline $15 \ldots$ & 2.55 & - - & - - & - - & -- & - - & - - & - - & - - & - - & - - & - & - \\
\hline $15 \ldots$ & -- & 0.50 & 23.6 & 565 & 8.6 & 8.8 & 8.08 & 0.020 & $<0.0 \odot 2$ & $<0.57$ & $\odot .027$ & 0.41 & 0.55 \\
\hline $15 \ldots$ & -- & 8.0 & 22.3 & 575 & 8.0 & 2.7 & - - & 0.033 & $\odot .0 \odot 2$ & - - & - - & - - & - - \\
\hline $15 \ldots$ & - - & 12.0 & 13.0 & 595 & 7.6 & 0.2 & - - & 0.178 & 0.146 & - - & -- & - - & - - \\
\hline
\end{tabular}




\section{DELAVAN LAKE AT CENTER NEAR DELAVAN LAKE, W}

WATER-QUALITY DATA, AUGUST 19 TO SEPTEMBER 9, 2014

(Milligrams per liter unless otherwise indicated)

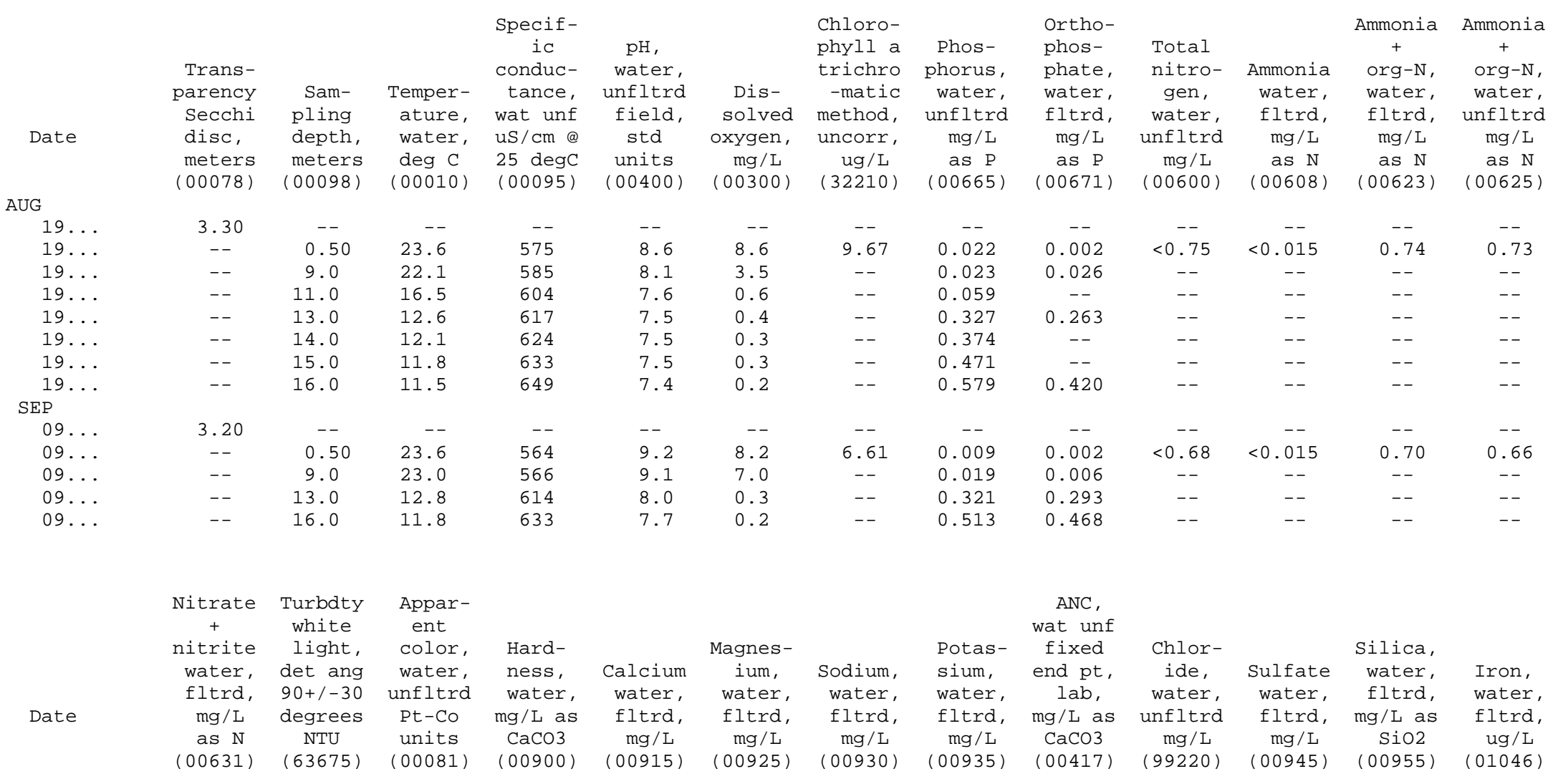

NOV 2013

19.

$19 \ldots$

MAR 2014

๑4...

$04 \ldots$

$0 . \overline{117}$

249

- -

-

$\begin{array}{ll} & \\ -- & -- \\ -- & -- \\ -- & -- \\ -- & -- \\ -- & --\end{array}$


423556088365001 DELAVAN LAKE AT CENTER NEAR DELAVAN LAKE, WI

WATER-QUALITY DATA, MAY 15 TO SEPTEMBER 9, 2014

(Milligrams per liter unless otherwise indicated)

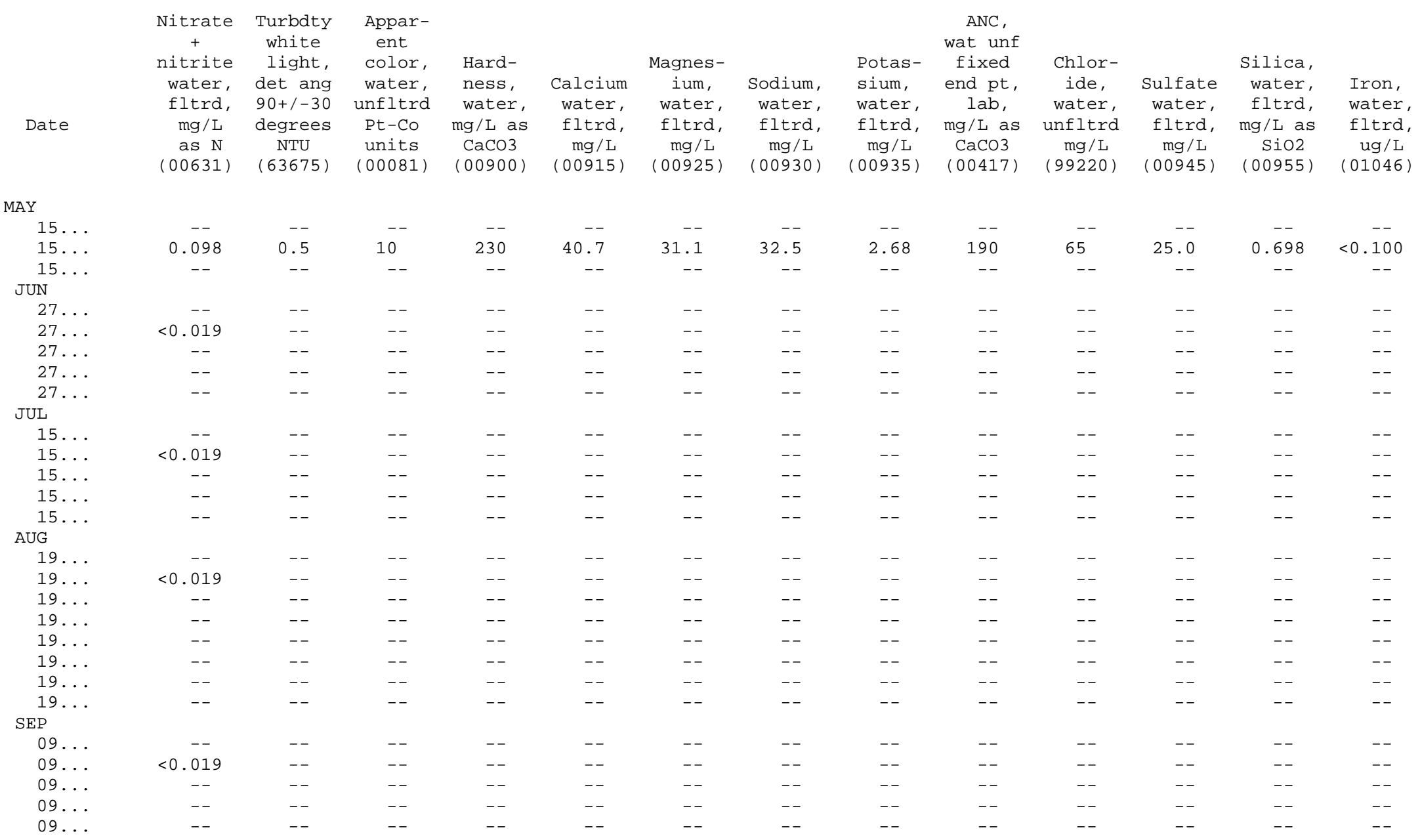


423556088365001 DELAVAN LAKE AT CENTER NEAR DELAVAN LAKE, WI

WATER-QUALITY DATA, NOVEMBER 13, 2013 TO MAY 15, 2014

(Milligrams per liter unless otherwise indicated)

$\begin{array}{ccc} & \begin{array}{c}\text { Dis- } \\ \text { solved } \\ \text { solids }\end{array} \\ \text { Manga- } & \text { dried @ } \\ \text { nese, } & \text { water, } 180 \text { degC } \\ \text { fltrd, } & \text { wat flt } \\ \text { ug/L } & \mathrm{mg} / \mathrm{L} \\ (01056) & (70300)\end{array}$

NOV 2013

$19 \ldots$

$19 \ldots$

MAR 2014

$\odot 4 \ldots$

$04 \ldots$

MAY

$15 \ldots$

15.

$15 \ldots$ 
423556088365001 DELAVAN LAKE AT CENTER NEAR DELAVAN LAKE, WI

LAKE-DEPTH PROFILES, NOVEMBER 19, 2013 TO JUNE 27, 2014

11-19-13
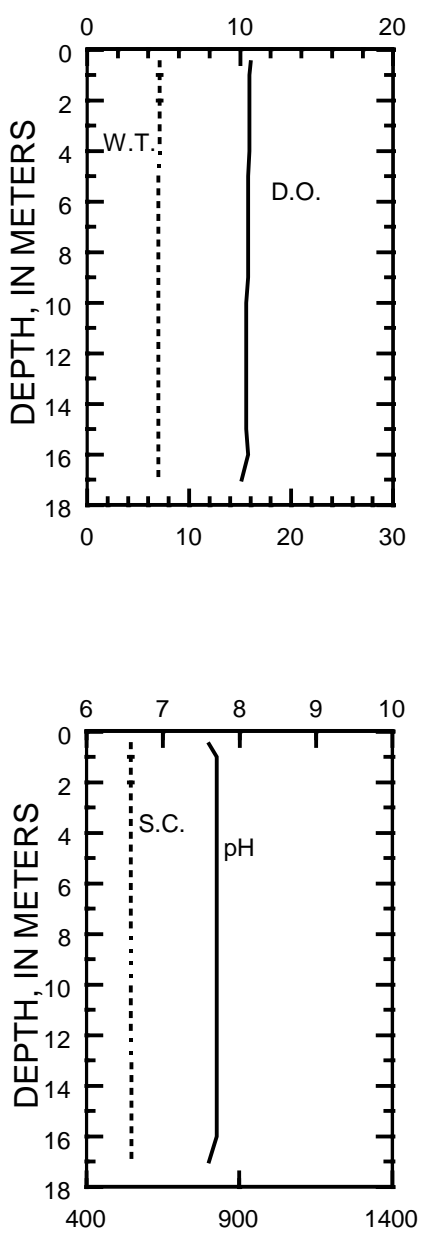

03-04-14

05-15-14

DISSOLVED OXYGEN (D.O.), IN MILLIGRAMS PER LITER
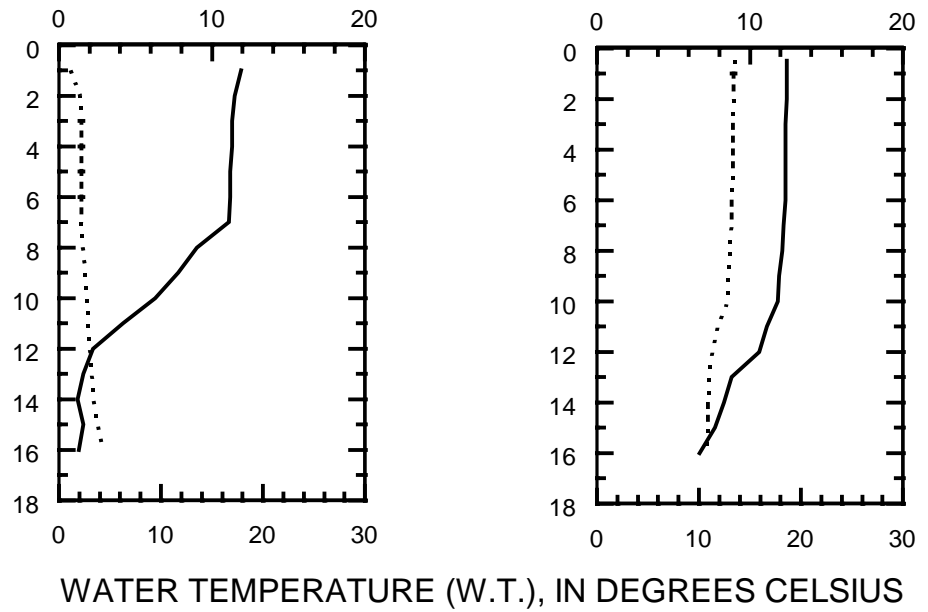

PH, IN STANDARD UNITS

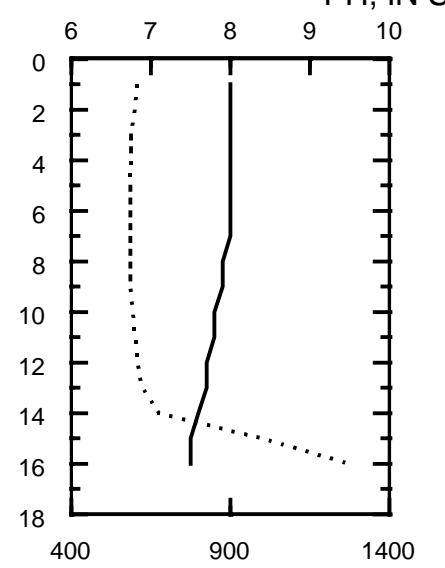

06-27-14
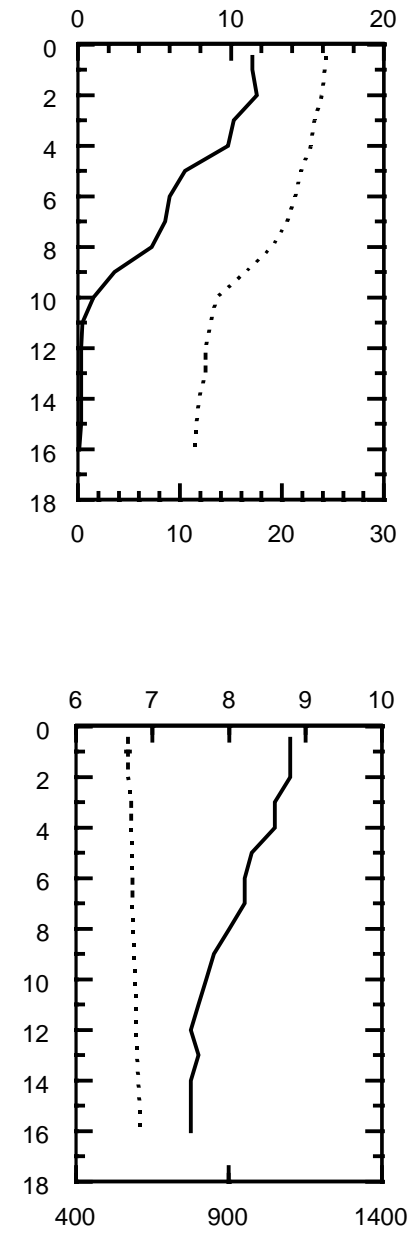

SPECIFIC CONDUCTANCE (S.C.), IN MICROSIEMENS PER CENTIMETER AT 25 DEGREES CELSIUS 
423556088365001 DELAVAN LAKE AT CENTER NEAR DELAVAN LAKE, WI

LAKE-DEPTH PROFILES, JULY 15 TO SEPTEMBER 9, 2014

$07-15-14$
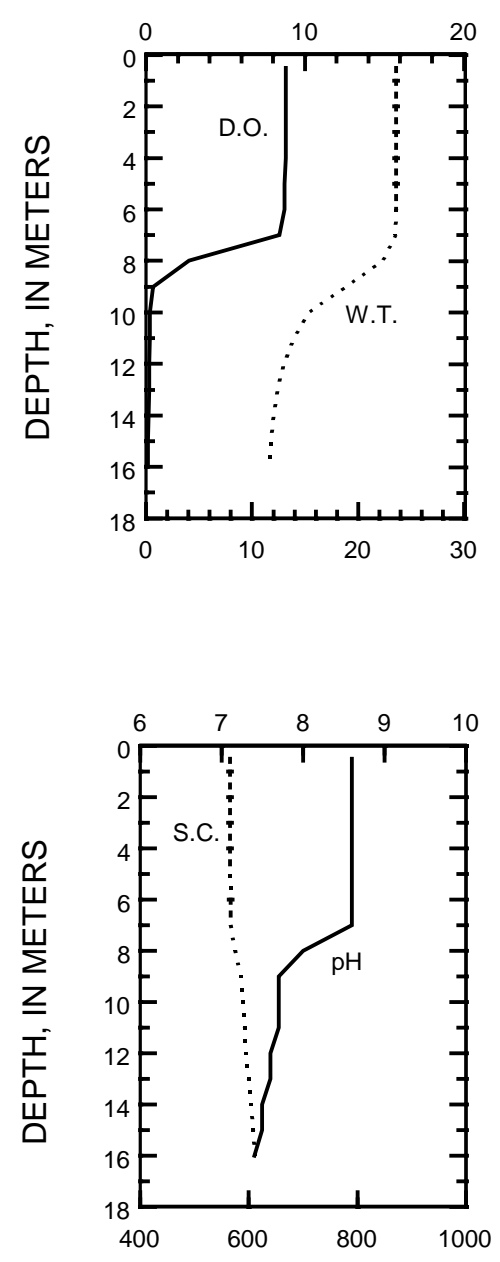

08-19-14

DISSOLVED OXYGEN (D.O.), IN MILLIGRAMS PER LITER

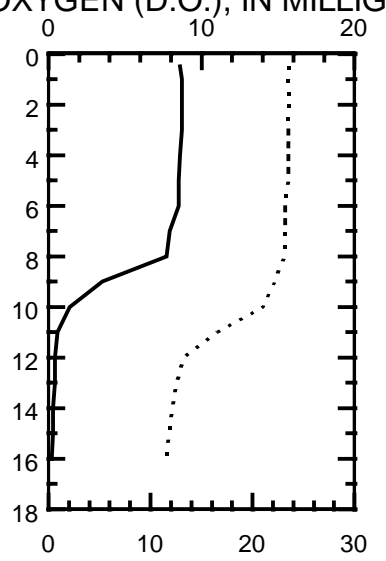

WATER TEMPERATURE (W.T.), IN DEGREES CELSIUS

$\mathrm{PH}$, IN STANDARD UNITS

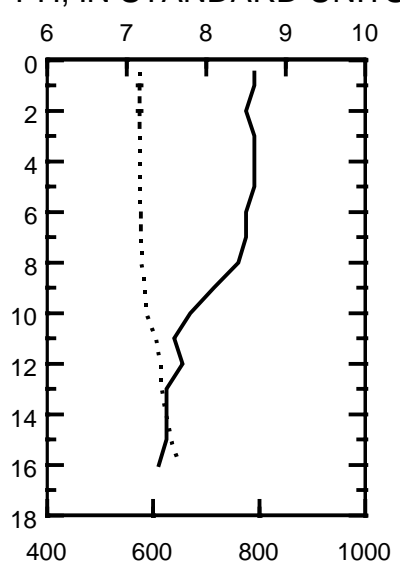

09-09-14
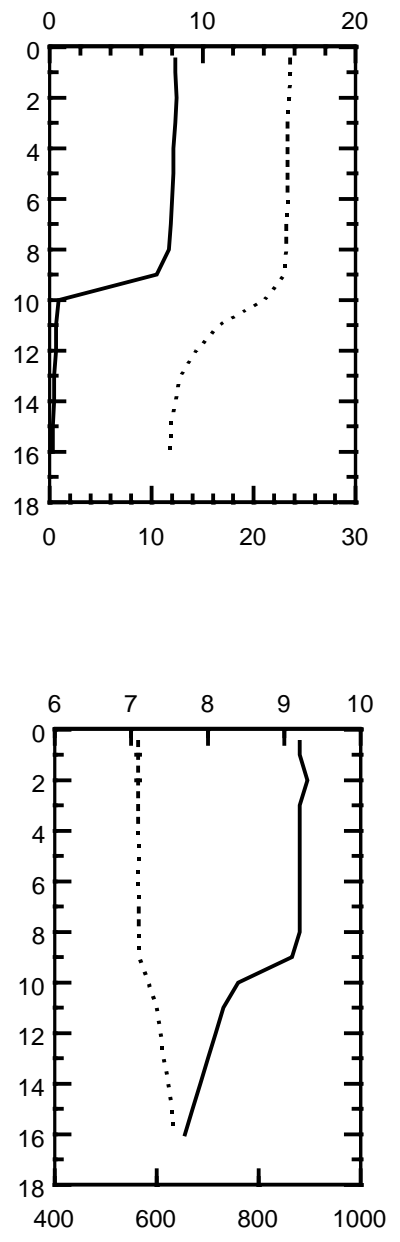

SPECIFIC CONDUCTANCE (S.C.), IN MICROSIEMENS PER CENTIMETER AT 25 DEGREES CELSIUS 

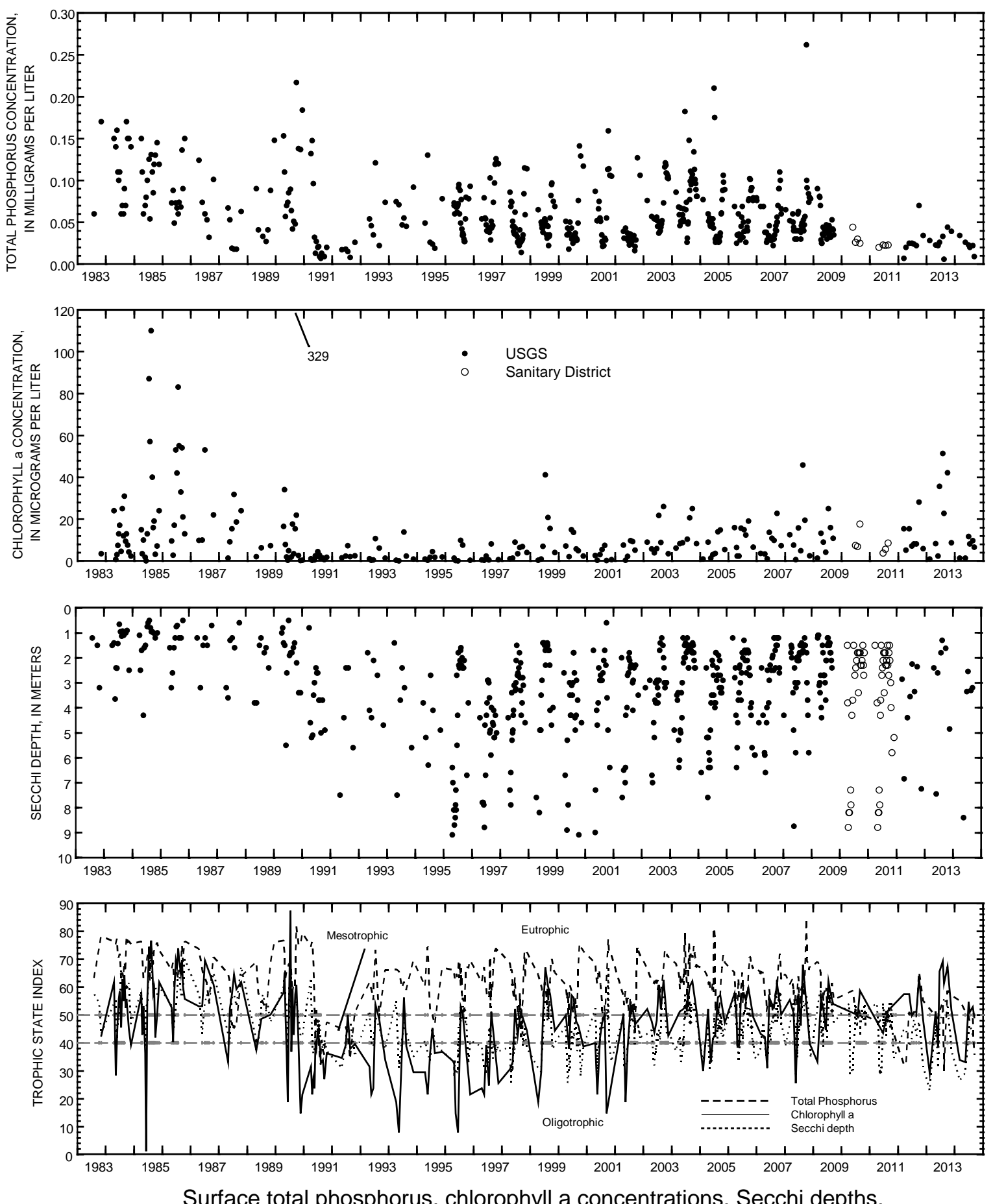

and TSI data for Delavan Lake, at Center, near Delavan, Wisconsin. 


\section{DELAVAN LAKE, AT NORTH END, NEAR LAKE LAWN, Wl}

LOCATION.--Lat $42^{\circ} 36^{\prime} 59^{\prime \prime}$, long $88^{\circ} 35^{\prime} 44^{\prime \prime}$, in NW 1/4 SW 1/4 Sec.22, T.2 N., R.16 E., Walworth County, Hydrologic Unit 07090001, 2.6 mi southeast of Delavan.

SURFACE AREA--3.24 $\mathrm{mi}^{2}$

DRAINAGE AREA.--41.4 $\mathrm{mi}^{2}$, of which $2.3 \mathrm{mi}^{2}$ is non-contributing.

PERIOD OF RECORD.--October 1983 to August 2009, March 2012 to September 2014.

WATER-QUALITY DATA, NOVEMBER 19, 2013 TO SEPTEMBER 9, 2014

(Milligrams per liter unless otherwise indicated)

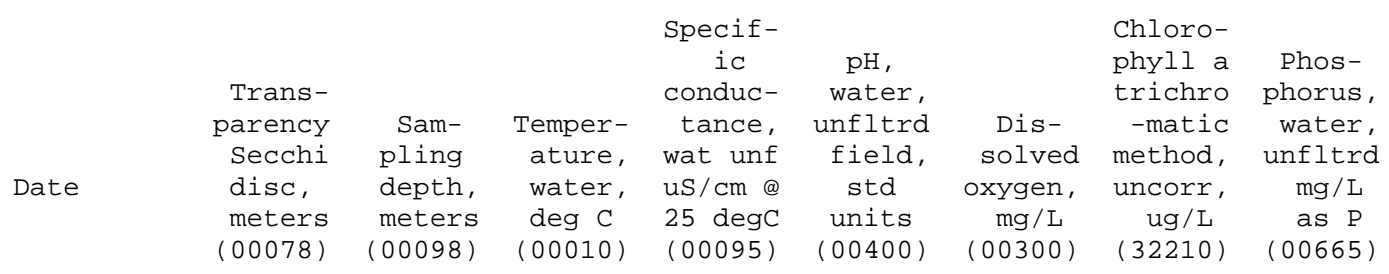

\begin{tabular}{|c|c|c|c|c|c|c|c|c|}
\hline \multicolumn{9}{|c|}{ NOV 2013} \\
\hline $19 \ldots$ & 4.60 & - - & - & - & - - & - & - - & - - \\
\hline $19 \ldots$ & - & 0.50 & 7.0 & 546 & 7.9 & 11.1 & - - & 0.037 \\
\hline \multicolumn{9}{|l|}{ MAR 2014} \\
\hline$\odot 4 \ldots$ & 2.60 & - - & - - & - - & - - & - - & - - & - - \\
\hline $04 \ldots$ & - & 1.0 & 1.7 & 592 & 8.0 & 12.6 & - - & 0.063 \\
\hline \multicolumn{9}{|l|}{ MAY } \\
\hline $15 \ldots$ & 5.40 & -- & -- & -- & - - & -- & -- & -- \\
\hline $15 \ldots$ & - - & 0.50 & 12.4 & 564 & 8.2 & 12.0 & 1.43 & 0.034 \\
\hline \multicolumn{9}{|l|}{ JUN } \\
\hline $27 \ldots$ & 2.35 & -- & -- & -- & - - & -- & - - & -- \\
\hline $27 \ldots$ & -- & 0.50 & 24.2 & 573 & 8.7 & 11.1 & 14.1 & 0.022 \\
\hline \multicolumn{9}{|l|}{ JUL } \\
\hline $15 \ldots$ & 2.05 & - - & - - & -- & - - & - & - & -- \\
\hline $15 \ldots$ & -- & 0.50 & 23.5 & 565 & 8.6 & 8.6 & 9.67 & 0.025 \\
\hline \multicolumn{9}{|l|}{ AUG } \\
\hline $19 \ldots$ & 3.30 & -- & -- & -- & - - & -- & -- & -- \\
\hline $19 \ldots$ & -- & 0.50 & 24.1 & 576 & 8.5 & 9.0 & 9.33 & 0.023 \\
\hline \multicolumn{9}{|l|}{ SEP } \\
\hline$\odot 9 \ldots$ & 2.55 & - & -- & - - & - - & - & - - & - - \\
\hline ०9. . & -- & 0.50 & 23.8 & 562 & 8.9 & 8.6 & 6.85 & 0.023 \\
\hline
\end{tabular}




\section{DELAVAN LAKE, AT NORTH END, NEAR LAKE LAWN, WI}

LAKE-DEPTH PROFILES, NOVEMBER 19, 2013 TO JUNE 27, 2014

$11-19-13$
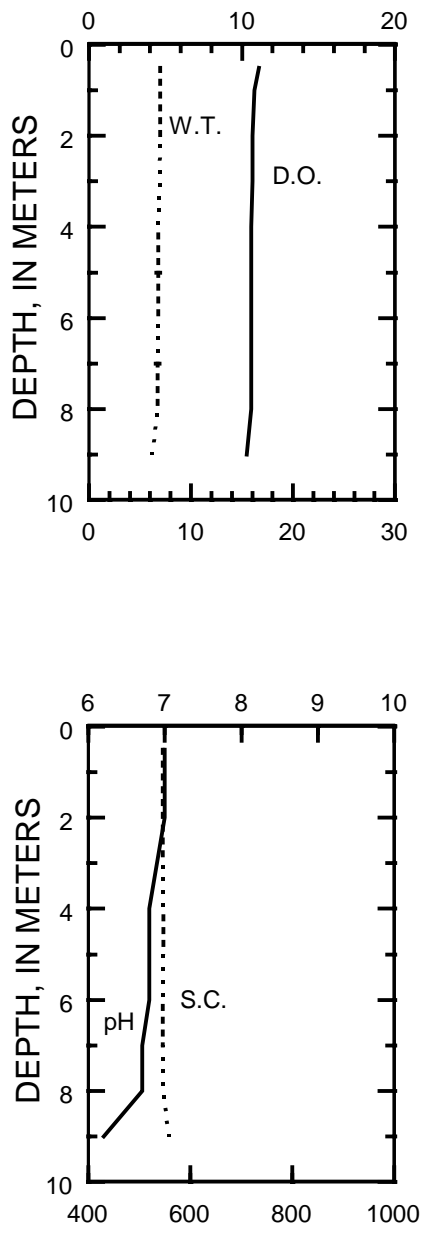

03-04-14

05-15-14

DISSOLVED OXYGEN (D.O.), IN MILLIGRAMS PER LITER
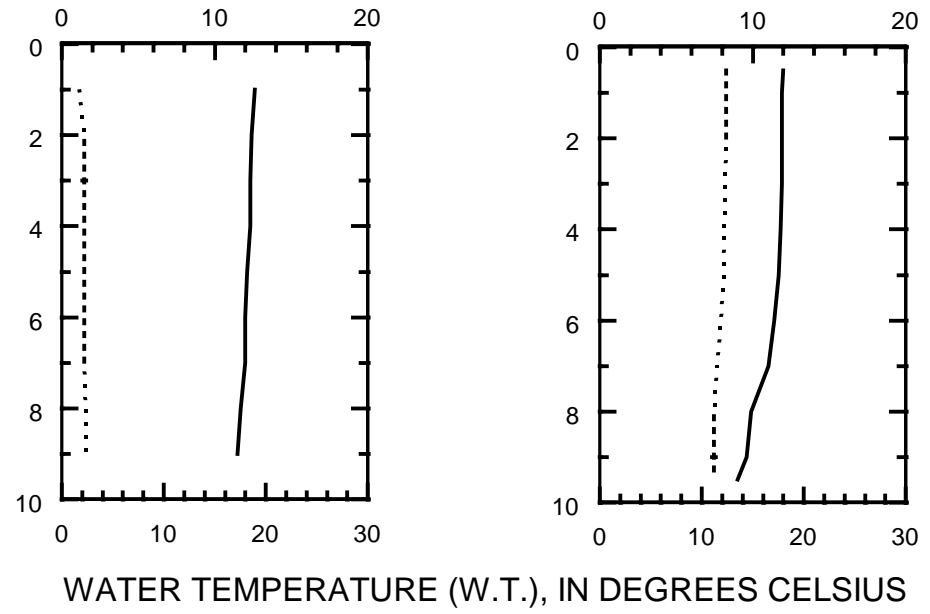

WATER TEMPERATURE (W.T.), IN DEGREES CELSIUS

PH, IN STANDARD UNITS
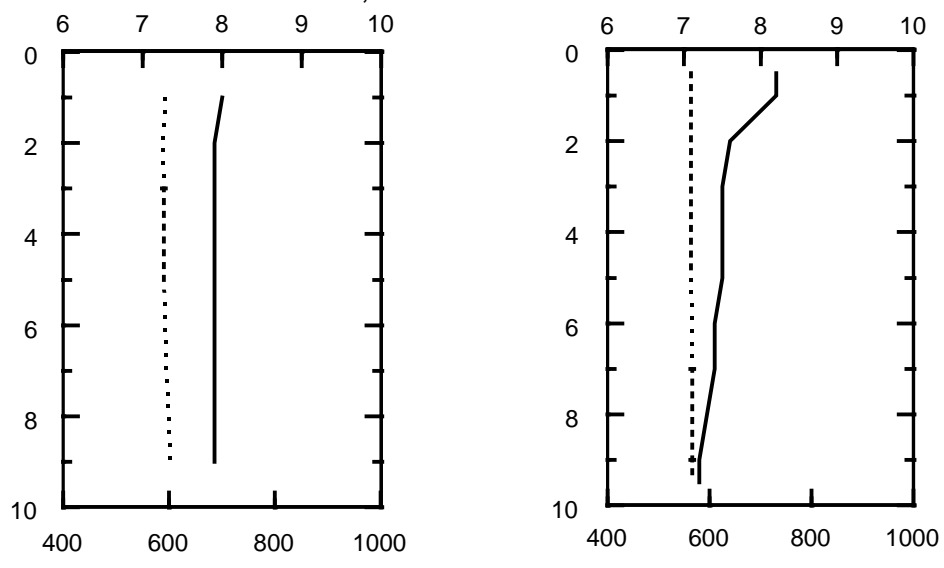

$06-27-14$
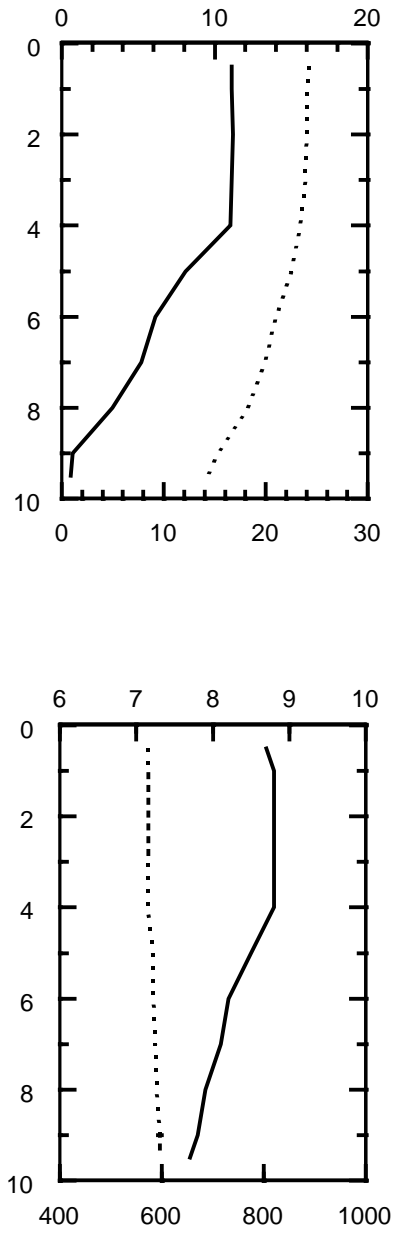

SPECIFIC CONDUCTANCE (S.C.), IN MICROSIEMENS PER CENTIMETER AT 25 DEGREES CELSIUS 


\section{DELAVAN LAKE, AT NORTH END, NEAR LAKE LAWN, Wl}

LAKE-DEPTH PROFILES, JULY 15 TO SEPTEMBER 9, 2014

07-15-14
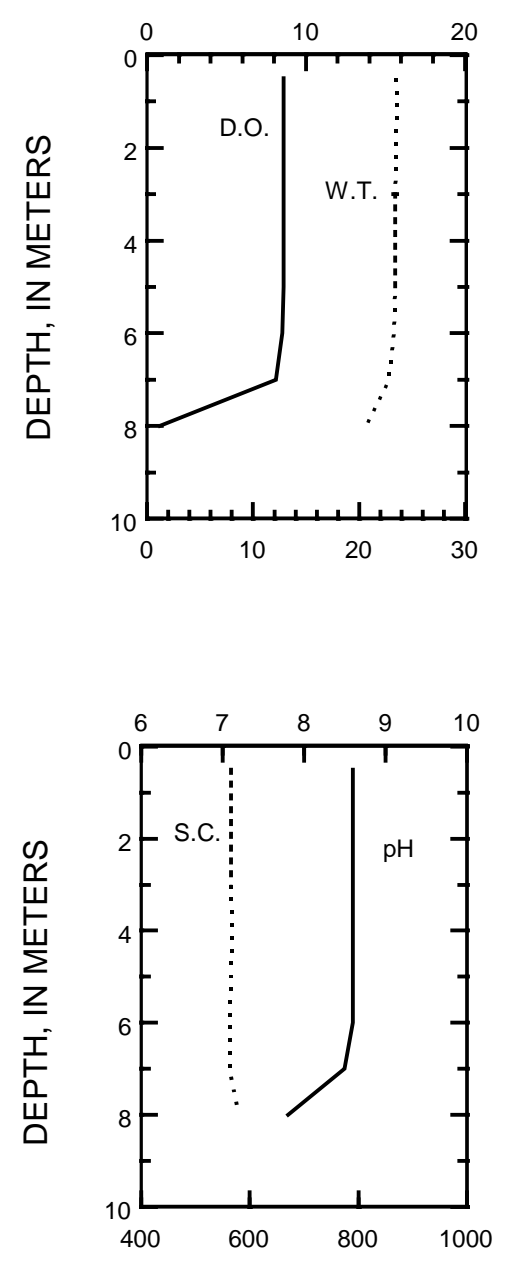

08-19-14

DISSOLVED OXYGEN (D.O.), IN MILLIGRAMS PER LITER

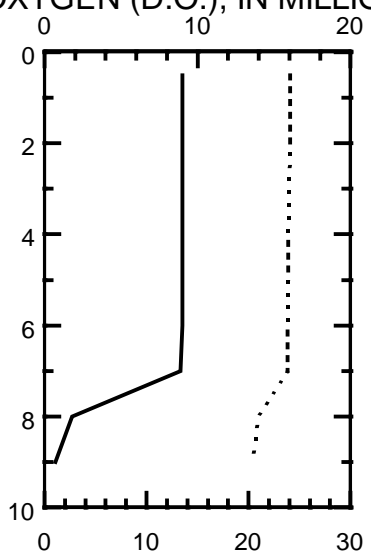

WATER TEMPERATURE (W.T.), IN DEGREES CELSIUS

$\mathrm{PH}$, IN STANDARD UNITS

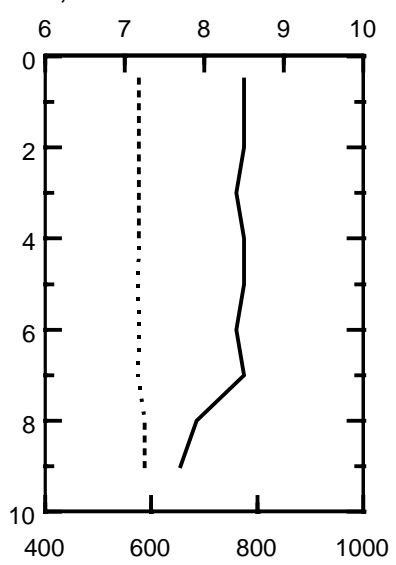

09-09-14
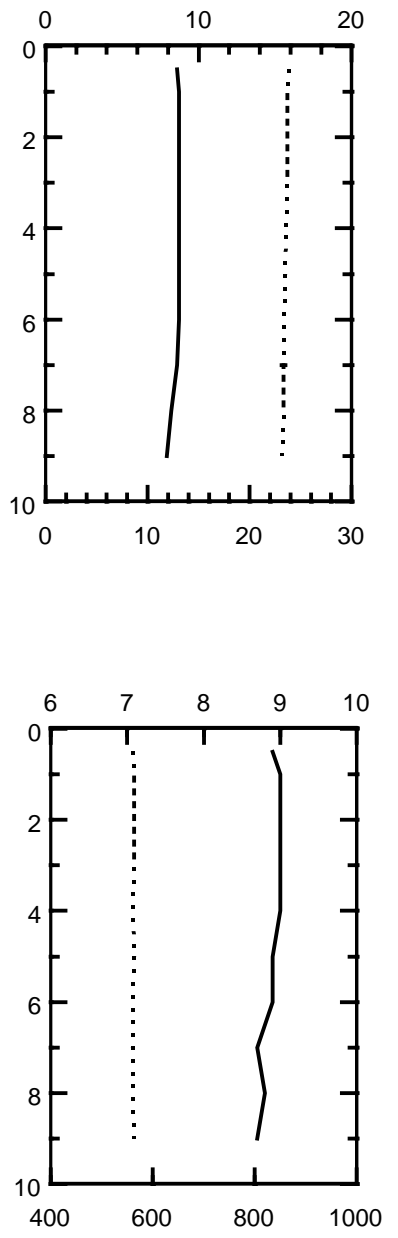

SPECIFIC CONDUCTANCE (S.C.), IN MICROSIEMENS PER CENTIMETER AT 25 DEGREES CELSIUS 


\section{DELAVAN LAKE, AT SW END, NEAR DELAVAN LAKE, WI}

LOCATION.--Lat 42³5'26", long 88³8'01", in SE 1/4 NW 1/4 Sec.32, T.2 N., R.16 E., Walworth County, Hydrologic Unit 07090001, 2.6 mi southeast of Delavan. SURFACE AREA--3.24 mi

DRAINAGE AREA.--41.4 mi², of which $2.3 \mathrm{mi}^{2}$ is non-contributing

PERIOD OF RECORD.-- October 1983 to August 2009, March 2012 to September 2014.

WATER-QUALITY DATA, NOVEMBER 19, 2013 TO SEPTEMBER 9, 2014

(Milligrams per liter unless otherwise indicated)

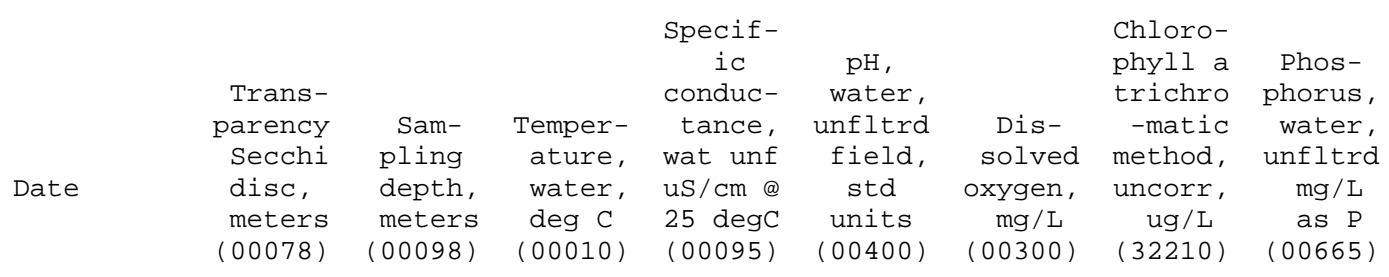

\begin{tabular}{|c|c|c|c|c|c|c|c|c|}
\hline \multicolumn{9}{|l|}{ NOV 2013} \\
\hline $19 \ldots$ & 5.05 & - - & - - & -- & -- & - - & - - & - - \\
\hline $19 \ldots$ & - - & 0.50 & 7.1 & 545 & 7.8 & 10.5 & - - & 0.037 \\
\hline \multicolumn{9}{|l|}{ MAR 2014} \\
\hline $04 \ldots$ & 2.30 & - - & - - & - - & -- & - - & - - & -- \\
\hline $04 \ldots$ & -- & 1.0 & 1.8 & 592 & 8.0 & 12.2 & - - & 0.051 \\
\hline \multicolumn{9}{|l|}{ MAY } \\
\hline $15 \ldots$ & 8.40 & - - & - - & - - & -- & - - & - - & - - \\
\hline $15 \ldots$ & - - & 0.50 & 13.7 & 563 & 8.3 & 12.5 & 1.34 & 0.026 \\
\hline \multicolumn{9}{|l|}{ JUN } \\
\hline $27 \ldots$ & 2.95 & - - & - - & - - & -- & - - & - - & - - \\
\hline $27 \ldots$ & -- & 0.50 & 25.0 & 570 & 8.8 & 11.0 & 10.5 & 0.022 \\
\hline \multicolumn{9}{|l|}{ JUL } \\
\hline $15 \ldots$ & 2.65 & - - & - - & - - & - - & - - & - - & - - \\
\hline $15 \ldots$ & -- & 0.50 & 23.5 & 564 & 8.6 & $9 . \odot$ & 7.37 & 0.020 \\
\hline \multicolumn{9}{|l|}{ AUG } \\
\hline $19 \ldots$ & 3.30 & - - & - - & - - & - - & - - & - - & -- \\
\hline $19 \ldots$ & -- & 0.50 & 23.0 & 574 & 8.5 & 7.6 & 8.26 & 0.020 \\
\hline \multicolumn{9}{|l|}{ SEP } \\
\hline $09 \ldots$ & 2.70 & - - & - - & - - & -- & - - & - - & - - \\
\hline $09 \ldots$ & -- & 0.50 & 23.5 & 563 & 9.0 & 8.2 & 6.95 & 0.022 \\
\hline
\end{tabular}




\section{DELAVAN LAKE, AT SW END, NEAR DELAVAN LAKE, WI}

LAKE-DEPTH PROFILES, NOVEMBER 19, 2013 TO JUNE 27, 2014

$11-19-13$
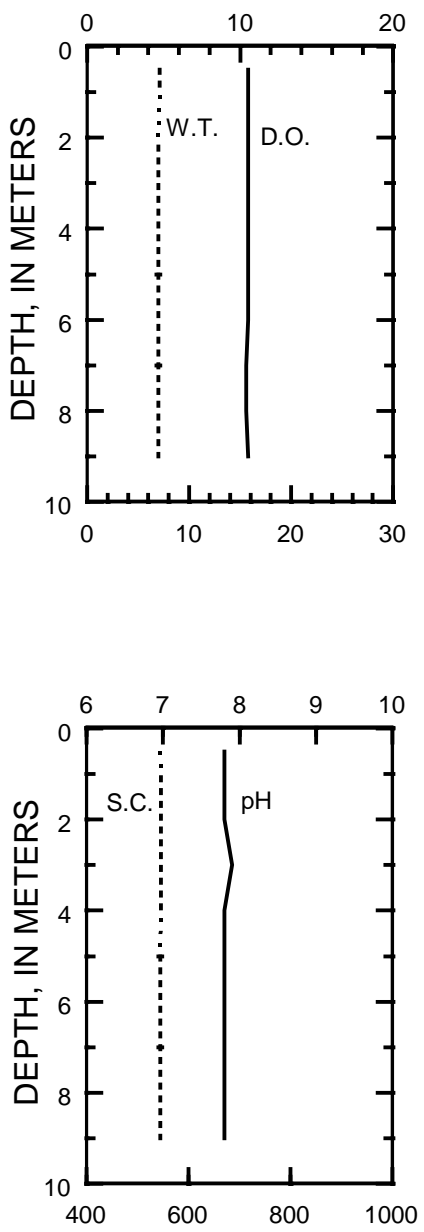

03-04-14

05-15-14

DISSOLVED OXYGEN (D.O.), IN MILLIGRAMS PER LITER
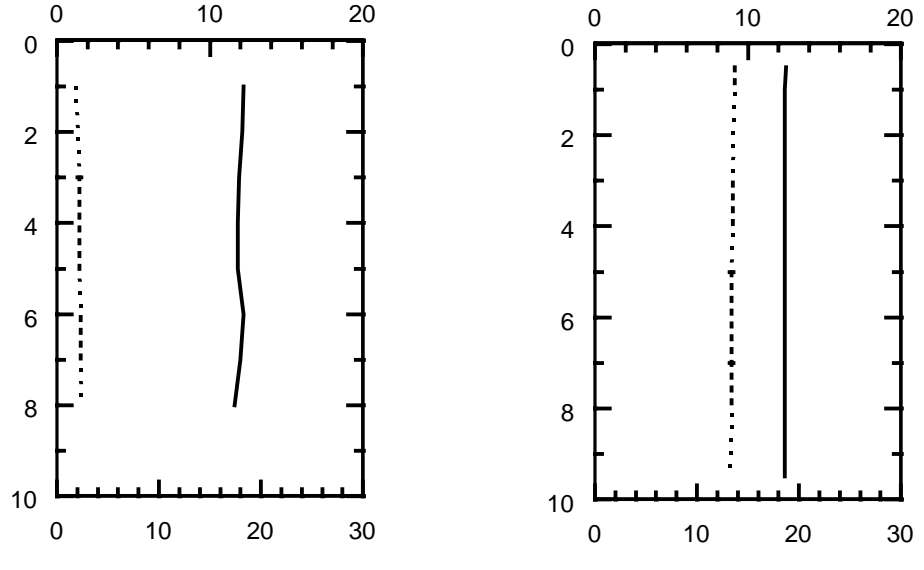

WATER TEMPERATURE (W.T.), IN DEGREES CELSIUS

PH, IN STANDARD UNITS
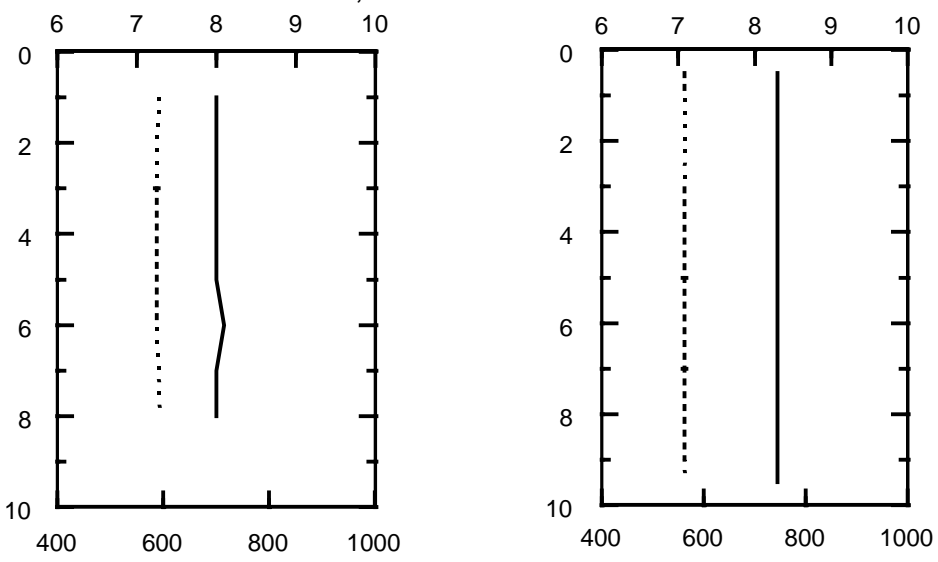

$06-27-14$
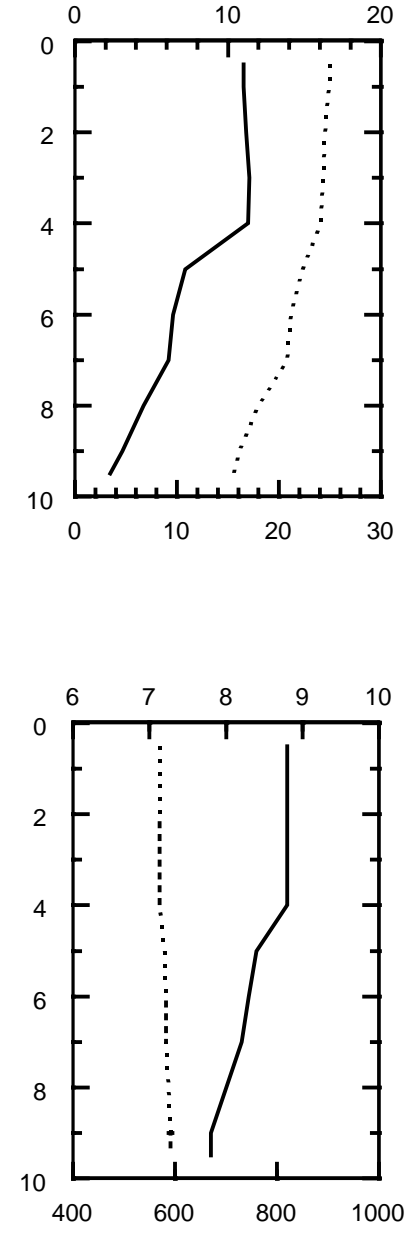

SPECIFIC CONDUCTANCE (S.C.), IN MICROSIEMENS PER CENTIMETER AT 25 DEGREES CELSIUS 


\section{DELAVAN LAKE, AT SW END, NEAR DELAVAN LAKE, WI}

LAKE-DEPTH PROFILES, JULY 15 TO SEPTEMBER 9, 2014

07-15-14
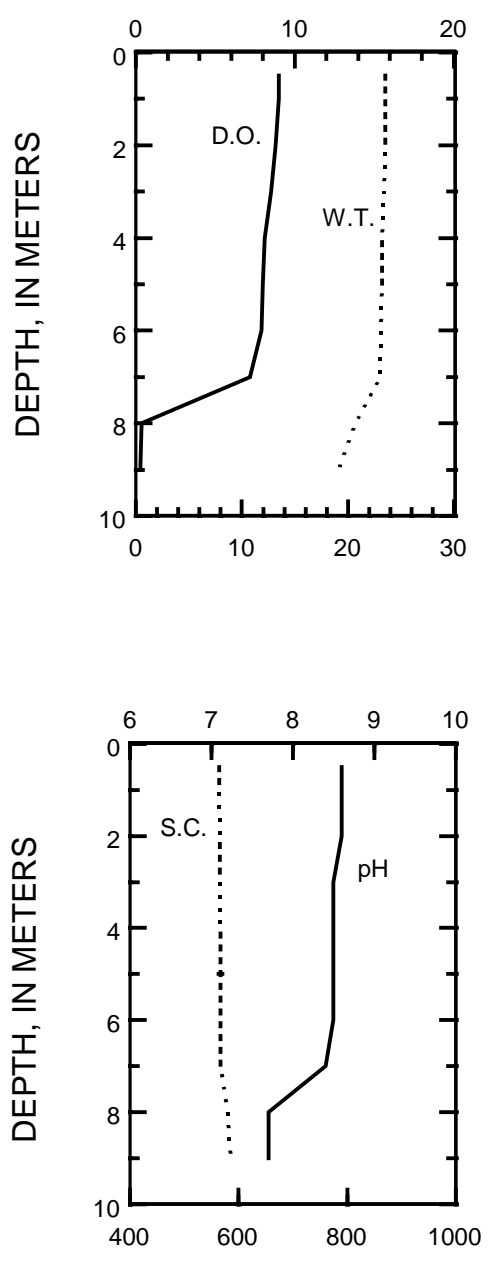

08-19-14

DISSOLVED OXYGEN (D.O.), IN MILLIGRAMS PER LITER

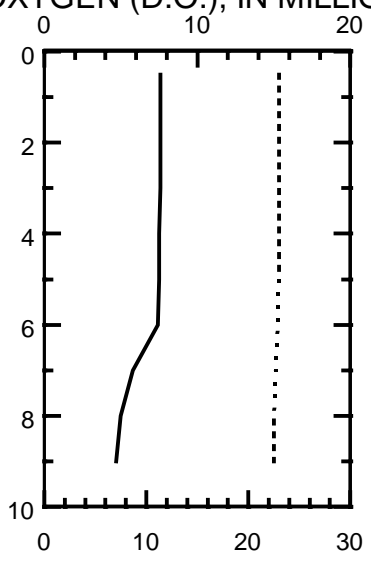

WATER TEMPERATURE (W.T.), IN DEGREES CELSIUS

$\mathrm{PH}$, IN STANDARD UNITS

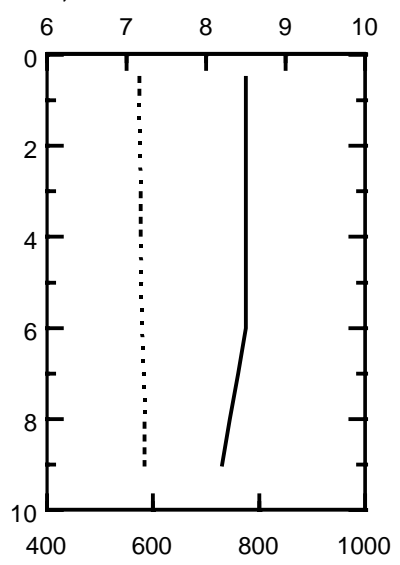

09-09-14
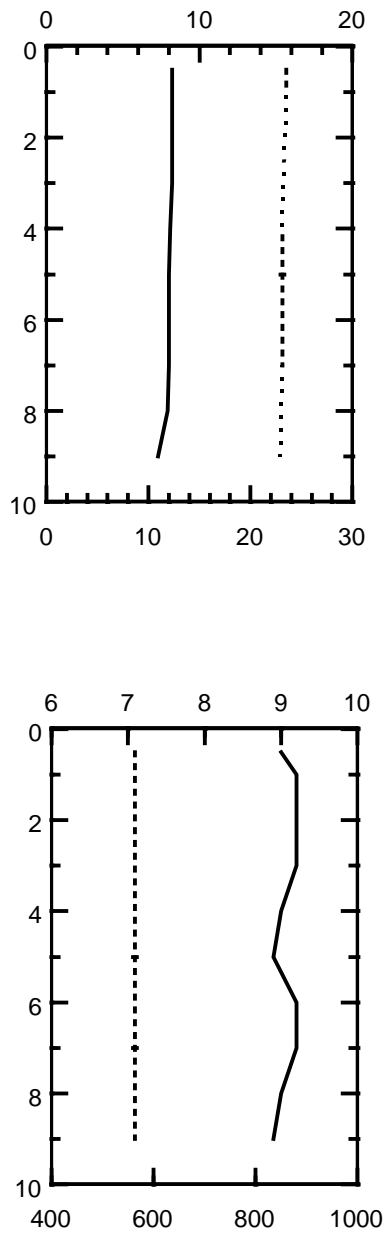

SPECIFIC CONDUCTANCE (S.C.), IN MICROSIEMENS PER CENTIMETER AT 25 DEGREES CELSIUS 
05404500 DEVILS LAKE NEAR BARABOO, WI

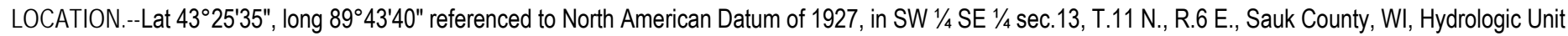
07070004, in Devils Lake State Park, 3.5 mi south of Baraboo.

SURFACE AREA.--0.56 $\mathrm{mi}^{2}$.

DRAINAGE AREA.--4.79 mi².

PERIOD OF RECORD.--June 1922 to August 1930, June to August 1932, June 1934 to September 1981 (fragmentary). October 1981 to September 1984 , data unpublished in district files. October 1984 to current year.

REVISED RECORDS.--WDR WI-78-1: Drainage area.

GAGE.--Water-stage recorder installed July 17, 1991. Datum of gage is $954.88 \mathrm{ft}$, above NAVD of 1988.

REMARKS.--Lake has no surface outlet. Water removed from lake by bottom withdrawal pipe, June 2-16, June 19 to July 8, and Sept. 4-30.

EXTREMES FOR PERIOD OF RECORD.--Maximum observed, $14.83 \mathrm{ft}$, June 12, 2008; minimum observed, 1.49 ft, Feb. 8, 1965.

\section{WATER YEAR OCTOBER 2013 TO SEPTEMBER 2014 \\ DAILY MEAN VALUES}

\begin{tabular}{|c|c|c|c|c|c|c|c|c|c|c|c|c|}
\hline DAY & OCT & NOV & DEC & JAN & FEB & MAR & APR & MAY & JUN & JUL & AUG & SEP \\
\hline 1 & 7.43 & 7.10 & 7.03 & 7.02 & 6.97 & 6.99 & 7.17 & 9.04 & 9.26 & 9.29 & 8.37 & 7.97 \\
\hline 2 & 7.41 & 7.09 & 7.03 & 7.02 & 6.97 & 6.99 & 7.17 & 9.07 & 9.46 & 9.24 & 8.36 & 7.95 \\
\hline 3 & 7.40 & 7.09 & 7.05 & 7.01 & 6.97 & 6.98 & 7.18 & 9.09 & 9.49 & 9.19 & 8.34 & 7.93 \\
\hline 4 & 7.41 & 7.08 & 7.05 & 7.01 & 6.97 & 6.99 & 7.19 & 9.09 & 9.48 & 9.15 & 8.33 & 7.97 \\
\hline 5 & 7.42 & 7.08 & 7.04 & 7.00 & 6.97 & 7.00 & 7.20 & 9.10 & 9.46 & 9.09 & 8.33 & 7.94 \\
\hline 6 & 7.41 & 7.20 & 7.03 & 6.99 & 6.97 & 7.00 & 7.20 & 9.10 & 9.42 & 9.04 & 8.31 & 7.89 \\
\hline 7 & 7.39 & 7.20 & 7.02 & 6.99 & 6.96 & 6.99 & 7.20 & 9.10 & 9.38 & 8.99 & 8.29 & 7.85 \\
\hline 8 & 7.38 & 7.19 & 7.01 & 6.99 & 6.96 & 6.99 & 7.22 & 9.11 & 9.33 & 8.96 & 8.26 & 7.82 \\
\hline 9 & 7.36 & 7.18 & 7.02 & 6.98 & 6.96 & 6.99 & 7.22 & 9.12 & 9.29 & 8.93 & 8.24 & 7.78 \\
\hline 10 & 7.34 & 7.16 & 7.01 & 6.98 & 6.96 & 6.98 & 7.21 & 9.11 & 9.24 & 8.90 & 8.21 & 7.77 \\
\hline 11 & 7.33 & 7.16 & 7.01 & 6.99 & 6.95 & 6.98 & 7.21 & 9.10 & 9.19 & 8.87 & 8.19 & 7.72 \\
\hline 12 & 7.31 & 7.14 & 7.01 & 6.99 & 6.96 & 6.99 & 7.22 & 9.17 & 9.14 & 8.85 & 8.16 & 7.69 \\
\hline 13 & 7.29 & 7.13 & 7.00 & 6.99 & 6.96 & 6.99 & 7.48 & 9.24 & 9.07 & 8.85 & 8.13 & 7.66 \\
\hline 14 & 7.27 & 7.11 & 7.00 & 7.00 & 6.96 & 6.98 & 8.15 & 9.24 & 9.01 & 8.82 & 8.10 & 7.63 \\
\hline 15 & 7.29 & 7.10 & 6.99 & 7.01 & 6.96 & 6.98 & 8.28 & 9.24 & 8.96 & 8.78 & 8.08 & 7.61 \\
\hline 16 & 7.29 & 7.11 & 6.99 & 7.00 & 6.96 & 6.98 & 8.33 & 9.22 & 8.91 & 8.77 & 8.06 & 7.60 \\
\hline 17 & 7.28 & 7.15 & 7.00 & 7.00 & 6.97 & 6.98 & 8.34 & 9.21 & 9.02 & 8.74 & 8.04 & 7.57 \\
\hline 18 & 7.26 & 7.17 & 7.00 & 7.00 & 6.98 & 6.98 & 8.36 & 9.20 & 9.20 & 8.72 & 8.02 & 7.55 \\
\hline 19 & 7.24 & 7.16 & 6.99 & 7.00 & 6.98 & 6.99 & 8.37 & 9.20 & 9.44 & 8.69 & 8.03 & 7.53 \\
\hline 20 & 7.23 & 7.15 & 7.00 & 7.00 & 6.99 & 6.99 & 8.38 & 9.19 & 9.52 & 8.67 & 8.02 & 7.53 \\
\hline 21 & 7.21 & 7.15 & 6.99 & 6.99 & 7.02 & 6.99 & 8.39 & 9.18 & 9.52 & 8.65 & 8.02 & 7.53 \\
\hline 22 & 7.19 & 7.14 & 7.01 & 6.99 & 7.01 & 6.99 & 8.39 & 9.16 & 9.50 & 8.63 & 8.02 & 7.51 \\
\hline 23 & 7.17 & 7.12 & 7.02 & 6.99 & 7.01 & 6.99 & 8.39 & 9.15 & 9.47 & 8.60 & 8.01 & 7.50 \\
\hline 24 & 7.15 & 7.11 & 7.01 & 6.98 & 7.00 & 6.99 & 8.44 & 9.13 & 9.43 & 8.57 & 7.99 & 7.48 \\
\hline 25 & 7.14 & 7.10 & 7.03 & 6.97 & 6.99 & 6.99 & 8.52 & 9.11 & 9.39 & 8.54 & 8.05 & 7.45 \\
\hline 26 & 7.11 & 7.08 & 7.03 & 6.99 & 6.99 & 6.98 & 8.54 & 9.09 & 9.35 & 8.50 & 8.05 & 7.43 \\
\hline 27 & 7.10 & 7.07 & 7.03 & 6.98 & 6.99 & 7.00 & 8.56 & 9.11 & 9.30 & 8.48 & 8.03 & 7.41 \\
\hline 28 & 7.08 & 7.06 & 7.03 & 6.98 & 6.99 & 7.08 & 8.65 & 9.18 & 9.25 & 8.45 & 8.01 & 7.39 \\
\hline 29 & 7.06 & 7.05 & 7.02 & 6.98 & --- & 7.10 & 8.86 & 9.16 & 9.24 & 8.42 & 7.99 & 7.37 \\
\hline 30 & 7.06 & 7.04 & 7.02 & 6.98 & --- & 7.11 & 8.99 & 9.13 & 9.30 & 8.40 & 7.99 & 7.35 \\
\hline 31 & 7.09 & --- & 7.02 & 6.97 & --- & 7.14 & --- & 9.11 & --- & 8.38 & 7.99 & --- \\
\hline MEAN & 7.26 & 7.12 & 7.02 & 6.99 & 6.98 & 7.00 & 7.93 & 9.14 & 9.30 & 8.78 & 8.13 & 7.65 \\
\hline MAX & 7.43 & 7.20 & 7.05 & 7.02 & 7.02 & 7.14 & 8.99 & 9.24 & 9.52 & 9.29 & 8.37 & 7.97 \\
\hline MIN & 7.06 & 7.04 & 6.99 & 6.97 & 6.95 & 6.98 & 7.17 & 9.04 & 8.91 & 8.38 & 7.99 & 7.35 \\
\hline
\end{tabular}




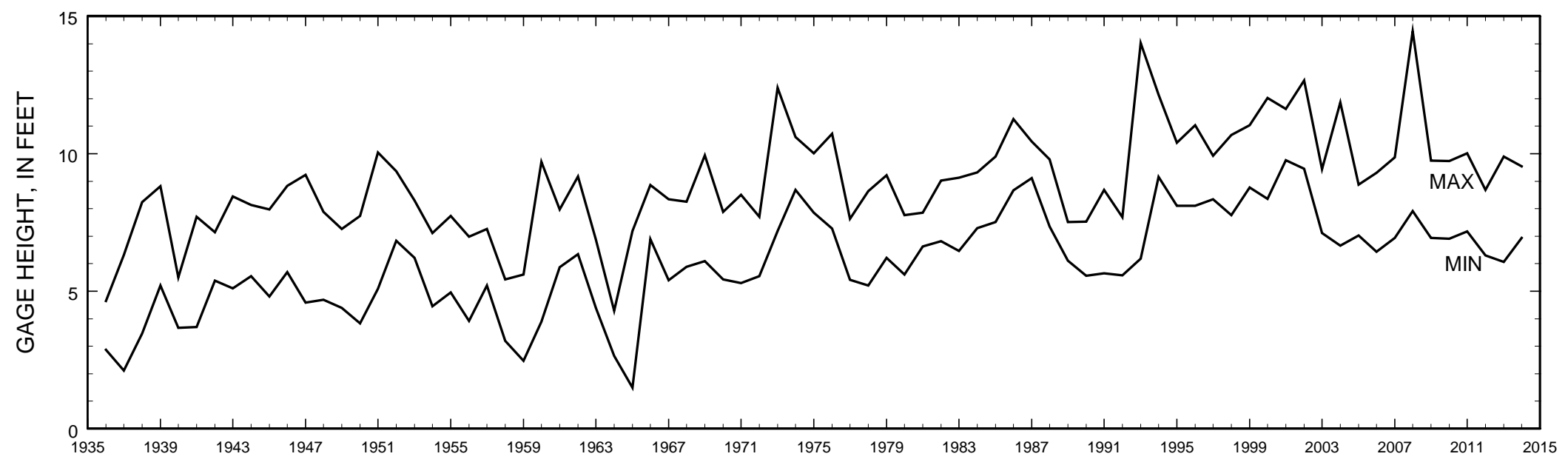

Annual minimum and maximum water levels for Devils Lake, 1936-2014. 


\section{GENEVA LAKE AT LAKE GENEVA, WI}

LOCATION.--Lat 42 $35^{\prime} 25^{\prime \prime}$, long $88^{\circ} 26^{\prime} 04^{\prime \prime}$ referenced to North American Datum of 1927, in SE 1/4 NW 1/4 Sec.36, T.2 N., R.17 E., Walworth County, WI, Hydrologic Unit 07120006, at Geneva Lake dam at Center Street at Lake Geneva.

SURFACE AREA.--8.22 mi².

DRAINAGE AREA.--28.7 $\mathrm{mi}^{2}$.

PERIOD OF RECORD.--October 1997 to August 2002, December 2002 to current year.

GAGE.--Water-stage recorder. Datum of gage is $861.86 \mathrm{ft}$ above NAVD of 1988 or $862.08 \mathrm{ft}$ above NGVD of 1929. Intermittent staff-gage readings during winter months.

REMARKS.--Records good except for estimated days, which are poor. Gage-height telemeter at station.

EXTREMES FOR PERIOD OF RECORD.--Maximum gage height, $3.47 \mathrm{ft}$, Apr. 17, 2013; minimum gage height, $1.38 \mathrm{ft}$, Oct. 13, 2012 (affected by wind).

EXTREMES FOR CURRENT YEAR.--Maximum gage height observed, $3.09 \mathrm{ft}$ (affected by wind), June 19; minimum gage height, $1.75 \mathrm{ft}$ (affected by wind), Oct. 20.

\section{GAGE HEIGHT, FEET \\ WATER YEAR OCTOBER 2013 TO SEPTEMBER 2014 DAILY MEAN VALUES}

[e, estimated]

\begin{tabular}{|c|c|c|c|c|c|c|c|c|c|c|c|c|}
\hline DAY & OCT & NOV & DEC & JAN & FEB & MAR & APR & MAY & JUN & JUL & AUG & SEP \\
\hline 1 & 2.27 & 1.93 & 2.16 & 2.33 & 2.50 & 2.31 & 2.44 & 2.64 & 2.61 & 2.83 & 2.41 & 2.58 \\
\hline 2 & 2.25 & 1.92 & 2.16 & 2.34 & 2.50 & 2.31 & 2.43 & 2.62 & 2.66 & 2.79 & 2.42 & 2.56 \\
\hline 3 & 2.25 & 1.91 & 2.18 & 2.34 & 2.50 & 2.29 & 2.43 & 2.62 & 2.64 & 2.78 & 2.42 & 2.54 \\
\hline 4 & 2.28 & 1.92 & 2.20 & 2.35 & 2.49 & 2.28 & 2.45 & 2.60 & 2.62 & 2.75 & 2.44 & 2.59 \\
\hline 5 & 2.33 & 1.92 & 2.22 & 2.37 & 2.50 & 2.29 & 2.45 & 2.58 & 2.62 & 2.73 & 2.52 & 2.60 \\
\hline 6 & 2.39 & 1.97 & 2.19 & 2.37 & 2.50 & 2.27 & 2.45 & 2.57 & 2.61 & 2.71 & 2.51 & 2.58 \\
\hline 7 & 2.35 & 1.97 & 2.18 & 2.37 & 2.50 & 2.26 & 2.45 & 2.56 & 2.60 & 2.71 & 2.50 & 2.56 \\
\hline 8 & 2.29 & 1.97 & 2.18 & 2.37 & 2.50 & 2.25 & 2.47 & 2.57 & 2.59 & 2.71 & 2.48 & 2.54 \\
\hline 9 & 2.26 & 1.98 & 2.22 & 2.38 & 2.50 & 2.24 & 2.47 & 2.61 & 2.57 & 2.67 & 2.47 & 2.52 \\
\hline 10 & 2.21 & 1.96 & 2.21 & 2.40 & 2.50 & 2.22 & 2.47 & 2.60 & 2.55 & 2.65 & 2.46 & 2.55 \\
\hline 11 & 2.18 & 1.96 & 2.19 & 2.46 & 2.49 & 2.21 & 2.47 & 2.59 & 2.62 & 2.63 & 2.47 & 2.51 \\
\hline 12 & 2.15 & 1.96 & 2.19 & 2.46 & 2.49 & 2.21 & 2.48 & 2.62 & 2.65 & 2.62 & 2.49 & 2.49 \\
\hline 13 & 2.11 & 1.98 & 2.16 & 2.47 & 2.49 & 2.20 & 2.51 & 2.80 & 2.62 & 2.64 & 2.48 & 2.48 \\
\hline 14 & 2.06 & 1.95 & 2.17 & 2.48 & 2.48 & 2.20 & 2.60 & 2.78 & 2.60 & 2.62 & 2.46 & 2.46 \\
\hline 15 & 2.04 & 1.93 & 2.18 & 2.49 & 2.45 & 2.20 & 2.61 & 2.76 & 2.60 & 2.59 & 2.45 & 2.44 \\
\hline 16 & 2.03 & 1.94 & 2.17 & 2.49 & 2.43 & 2.19 & 2.59 & 2.75 & 2.58 & 2.56 & 2.45 & 2.43 \\
\hline 17 & 1.99 & 2.07 & 2.19 & 2.49 & 2.42 & 2.19 & 2.59 & 2.73 & 2.70 & 2.55 & 2.42 & 2.42 \\
\hline 18 & 1.97 & 2.16 & 2.18 & 2.49 & 2.42 & 2.20 & 2.58 & 2.71 & 2.80 & 2.54 & 2.43 & 2.40 \\
\hline 19 & 1.93 & 2.15 & 2.18 & 2.50 & 2.41 & 2.23 & 2.57 & 2.69 & 2.90 & 2.53 & 2.49 & 2.41 \\
\hline 20 & 1.88 & 2.15 & 2.20 & 2.49 & 2.43 & 2.25 & 2.57 & 2.68 & 2.95 & 2.53 & 2.49 & 2.41 \\
\hline 21 & 1.90 & 2.17 & 2.20 & 2.50 & 2.46 & 2.26 & 2.57 & 2.67 & 2.93 & 2.52 & 2.50 & 2.40 \\
\hline 22 & 1.90 & 2.19 & 2.26 & 2.49 & 2.43 & 2.28 & 2.57 & 2.66 & 2.91 & 2.52 & 2.52 & 2.38 \\
\hline 23 & 1.89 & 2.18 & 2.28 & 2.50 & 2.41 & 2.29 & 2.55 & 2.64 & 2.88 & 2.49 & 2.53 & 2.38 \\
\hline 24 & 1.88 & 2.19 & 2.27 & 2.49 & 2.39 & 2.30 & 2.55 & 2.63 & 2.86 & 2.48 & 2.54 & 2.37 \\
\hline 25 & 1.88 & 2.20 & 2.28 & 2.49 & e2.37 & 2.31 & 2.56 & 2.62 & 2.83 & 2.47 & 2.58 & 2.37 \\
\hline 26 & 1.85 & 2.17 & 2.28 & 2.49 & 2.36 & 2.31 & 2.55 & 2.62 & 2.79 & 2.45 & 2.62 & 2.37 \\
\hline 27 & 1.84 & 2.16 & 2.29 & 2.49 & 2.33 & 2.33 & 2.52 & 2.62 & 2.77 & 2.47 & 2.62 & 2.37 \\
\hline 28 & 1.81 & 2.16 & 2.29 & 2.49 & 2.31 & 2.39 & 2.56 & 2.64 & 2.76 & 2.44 & 2.60 & 2.37 \\
\hline 29 & 1.81 & 2.15 & 2.30 & 2.49 & --- & 2.40 & 2.62 & 2.62 & 2.77 & 2.43 & 2.61 & 2.37 \\
\hline 30 & 1.82 & 2.16 & 2.30 & 2.48 & --- & 2.41 & 2.64 & 2.61 & 2.79 & 2.42 & 2.62 & 2.35 \\
\hline 31 & 1.89 & --- & 2.31 & 2.48 & --- & 2.42 & --- & 2.61 & --- & 2.42 & 2.59 & --- \\
\hline MEAN & 2.05 & 2.05 & 2.22 & 2.45 & 2.45 & 2.27 & 2.53 & 2.65 & 2.71 & 2.59 & 2.50 & 2.46 \\
\hline MAX & 2.39 & 2.20 & 2.31 & 2.50 & 2.50 & 2.42 & 2.64 & 2.80 & 2.95 & 2.83 & 2.62 & 2.60 \\
\hline MIN & 1.81 & 1.91 & 2.16 & 2.33 & 2.31 & 2.19 & 2.43 & 2.56 & 2.55 & 2.42 & 2.41 & 2.35 \\
\hline
\end{tabular}




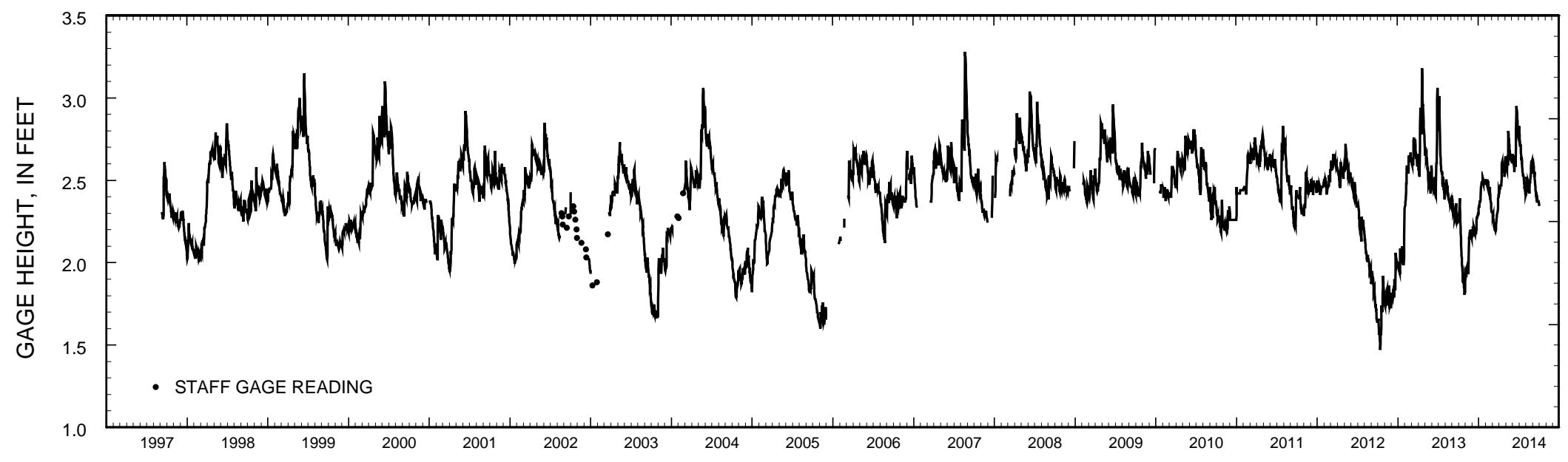

Stage hydrograph for Geneva Lake, 1997-2014. 


\section{GENEVA LAKE AT WEST END NEAR WILLIAMS BAY, W}

LOCATION.--Lat 42³3'29", long 88³2'33", in NE 1/4 SE 1/4 sec.12, T.1 N., R.16 E., Walworth County, Hydrologic Unit 07120006, 1.3 mi south of Williams Bay.

SURFACE AREA.--8.22 mi².

DRAINAGE AREA.--28.7 mi²

PERIOD OF RECORD.--April 1997 to current year.

REMARKS.--Lake sampled at deep hole at a depth of about $43 \mathrm{~m}$. Water-quality analyses done by Wisconsin State Laboratory of Hygiene. Samples for determination of chlorophyll a concentration are collected from the top $0.5 \mathrm{~m}$ of the lake.

WATER-QUALITY DATA, NOVEMBER 19, 2013 TO SEPTEMBER 9, 2014 (Milligrams per liter unless otherwise indicated)

\begin{tabular}{|c|c|c|c|c|c|c|c|c|c|c|c|c|c|}
\hline Date & $\begin{array}{c}\text { Trans- } \\
\text { parency } \\
\text { Secchi } \\
\text { disc, } \\
\text { meters } \\
(\odot \odot \odot 78)\end{array}$ & $\begin{array}{c}\text { Sam- } \\
\text { pling } \\
\text { depth, } \\
\text { meters } \\
(\odot \odot \odot 98)\end{array}$ & $\begin{array}{c}\text { Temper- } \\
\text { ature, } \\
\text { water, } \\
\text { deg C } \\
(00010)\end{array}$ & $\begin{array}{c}\text { Specif- } \\
\text { ic } \\
\text { conduc- } \\
\text { tance, } \\
\text { wat unf } \\
\text { uS/cm @ } \\
25 \text { degc } \\
(0 \odot \odot 95)\end{array}$ & $\begin{array}{c}\text { pH, } \\
\text { water, } \\
\text { unfltrd } \\
\text { field, } \\
\text { std } \\
\text { units } \\
(00400)\end{array}$ & $\begin{array}{c}\text { Dis- } \\
\text { solved } \\
\text { oxygen, } \\
\text { mg/L } \\
(0030 \odot)\end{array}$ & $\begin{array}{c}\text { Chloro- } \\
\text { phyll a } \\
\text { trichro } \\
\text {-matic } \\
\text { method, } \\
\text { uncorr, } \\
\text { ug/L } \\
(32210)\end{array}$ & $\begin{array}{c}\text { Phos- } \\
\text { phorus, } \\
\text { water, } \\
\text { unfltrd } \\
\text { mg/L } \\
\text { as } P \\
(00665)\end{array}$ & $\begin{array}{l}\text { Ortho- } \\
\text { phos- } \\
\text { phate, } \\
\text { water, } \\
\text { fltrd, } \\
\text { mg/L } \\
\text { as P } \\
(00671)\end{array}$ & $\begin{array}{c}\text { Total } \\
\text { nitro- } \\
\text { gen, } \\
\text { water, } \\
\text { unfltrd } \\
\text { mg/L } \\
(00600)\end{array}$ & $\begin{array}{c}\text { Ammonia } \\
\text { water, } \\
\text { fltrd, } \\
\text { mg/L } \\
\text { as N } \\
(0 \odot 608)\end{array}$ & $\begin{array}{c}\text { Ammonia } \\
+ \\
\text { org-N, } \\
\text { water, } \\
\text { fltrd, } \\
\text { mg/L } \\
\text { as N } \\
(00623)\end{array}$ & $\begin{array}{c}\text { Ammonia } \\
+ \\
\text { org-N, } \\
\text { water, } \\
\text { unfltrd } \\
\text { mg/L } \\
\text { as N } \\
(00625)\end{array}$ \\
\hline \multicolumn{14}{|l|}{ NOV 2013} \\
\hline $19 \ldots$ & 8.45 & - - & - - & - - & - - & - - & - - & - - & - - & - - & - - & - - & - - \\
\hline $04 \ldots$ & - - & 1.0 & 0.7 & 547 & 8.3 & 13.9 & - - & 0.017 & - - & - - & - & - & - - \\
\hline $04 \ldots$ & - - & 30.0 & 2.2 & 549 & 8.2 & 11.3 & - - & 0.016 & - - & - - & - - & - - & - - \\
\hline \multicolumn{14}{|l|}{ MAY } \\
\hline $13 \ldots$ & 4.90 & - - & - - & - - & - - & - - & - - & - - & - - & - - & - - & - - & - - \\
\hline $13 \ldots$ & - - & 0.50 & 10.7 & 531 & 8.5 & 12.6 & 3.32 & 0.010 & $\odot . \odot \odot 2$ & 0.36 & $<0.015$ & - & 0.34 \\
\hline \multicolumn{14}{|l|}{ JUN } \\
\hline $26 \ldots$ & 3.95 & -- & - - & -- & -- & -- & -- & -- & - - & - - & - - & - & - \\
\hline $26 \ldots$ & - - & 0.50 & 23.1 & 530 & 8.6 & 9.3 & 2.38 & 0.011 & $<0.0 \odot 2$ & $<0.42$ & $<0.015$ & 0.50 & $0.4 \odot$ \\
\hline $26 \ldots$ & - - & 7.0 & 22.6 & 530 & 8.7 & 9.3 & - - & 0.013 & - - & - - & - - & - - & - - \\
\hline $26 \ldots$ & -- & 38.0 & 6.9 & 544 & 8.0 & 7.8 & -- & 0.014 & - - & -- & -- & - - & -- \\
\hline $26 \ldots$ & -- & 42.0 & 6.8 & 545 & 8.0 & 6.9 & -- & $\odot .02 \odot$ & -- & -- & - & -- & -- \\
\hline \multicolumn{14}{|l|}{ JUL } \\
\hline $29 \ldots$ & 7.60 & -- & - - & -- & -- & -- & -- & -- & -- & - - & -- & -- & -- \\
\hline $29 \ldots$ & - - & 0.50 & 22.6 & 522 & 8.5 & 8.5 & 2.65 & 0.011 & $\odot .0 \odot 9$ & $<0.4 \odot$ & $<0.015$ & 0.34 & 0.38 \\
\hline $29 \ldots$ & - - & 8.0 & 22.6 & 522 & 8.6 & 8.4 & -- & 0.011 & - - & - & - - & - & - - \\
\hline $29 \ldots$ & -- & 13.0 & 11.1 & 525 & 8.3 & 9.7 & -- & 0.012 & - & -- & - & -- & -- \\
\hline $29 \ldots$ & -- & 35.0 & 7.0 & 531 & 7.5 & 6.5 & -- & 0.010 & - - & - - & - - & - - & - - \\
\hline $29 \ldots$ & - - & 38.0 & 6.9 & 531 & 7.4 & 4.9 & - - & 0.011 & - - & - - & - & - & - - \\
\hline $29 \ldots$ & - - & 42.0 & 6.8 & 535 & 7.3 & 2.8 & - - & $\odot . \odot 21$ & $<0.002$ & 0.59 & $\odot .056$ & 0.45 & 0.41 \\
\hline
\end{tabular}




\section{GENEVA LAKE AT WEST END NEAR WILLIAMS BAY, WI}

WATER-QUALITY DATA, NOVEMBER 19, 2013 TO SEPTEMBER 9, 2014 (Milligrams per liter unless otherwise indicated)

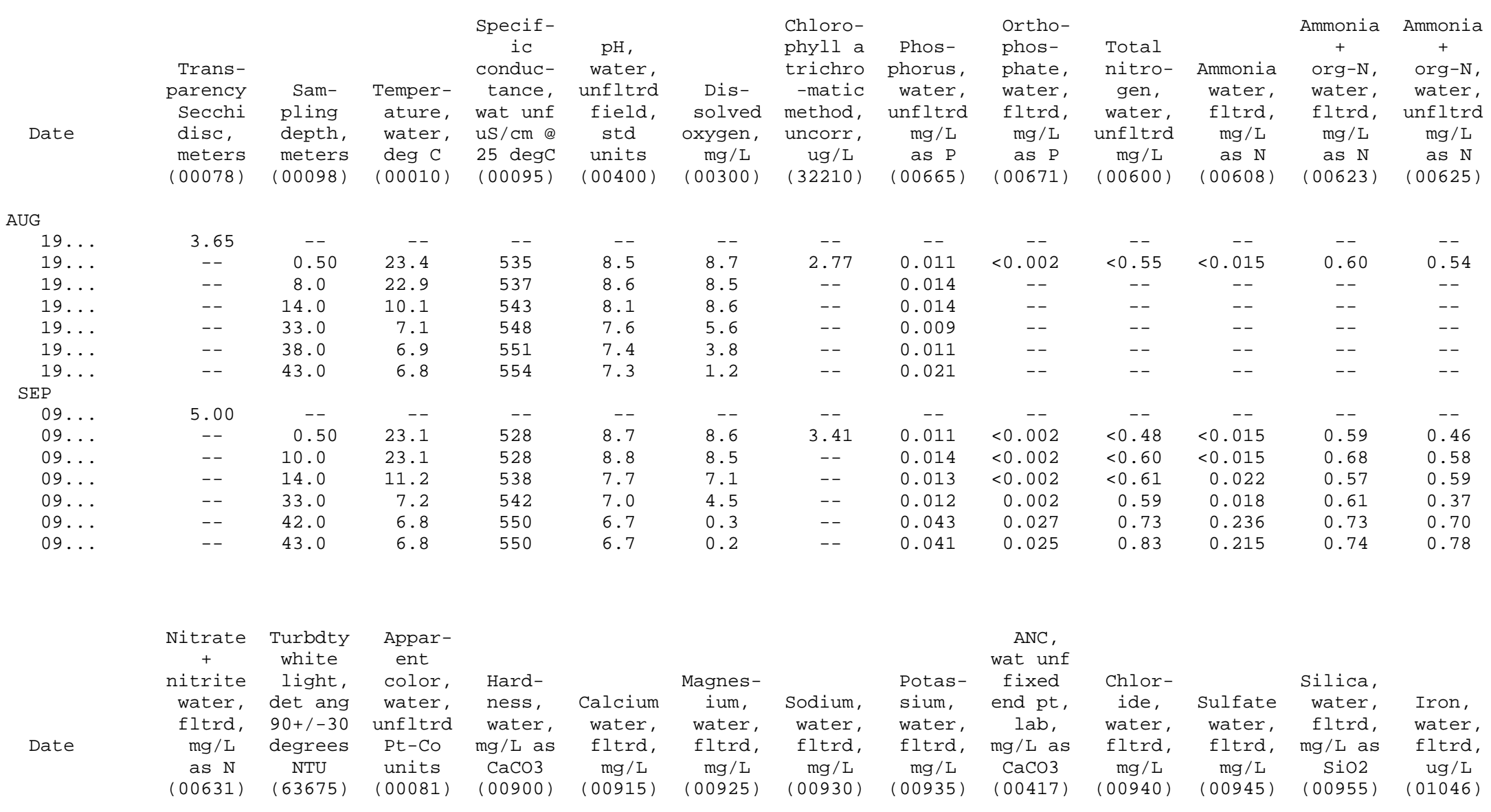

NOV 2013

$19 \ldots$

$19 \ldots$

$\odot .078$

04.

๑4...

MAY

$13 \ldots$

JUN

$26 \ldots$

$26 \ldots$

$26 \ldots$

$26 \ldots$

$-$

$-$

$0 . \overline{5}$

$<0 . \overline{-} 019$

- -

$26 \ldots$

- -

236

34.4

36.4

22.7

2.05

199

46

27.5

$2.50<0.100$

$\begin{array}{ll}- & - \\ -- & -\end{array}$

$\begin{array}{ll}- & - \\ - & -\end{array}$

- -

- -

$--$

$-$ 
423329088323300 GENEVA LAKE AT WEST END NEAR WILLIAMS BAY, WI

WATER-QUALITY DATA, NOVEMBER 19, 2013 TO SEPTEMBER 9, 2014 (Milligrams per liter unless otherwise indicated)

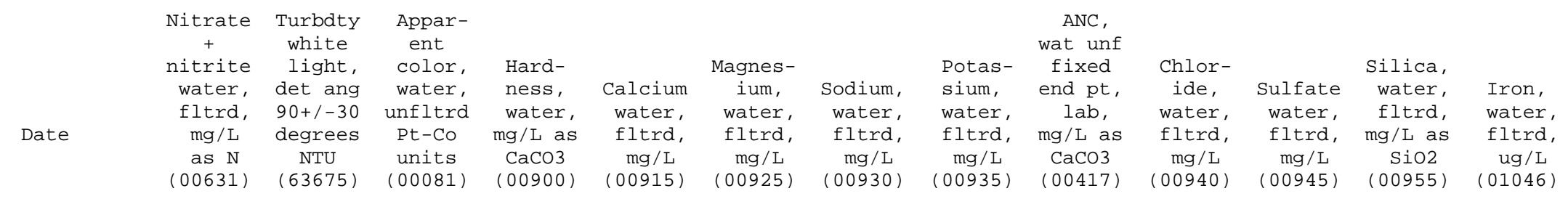

JUL

29.

$29 \ldots$

$29 \ldots$

29.

$29 \ldots$

$29 .$.

AUG

$19 \ldots$

$19 \ldots$

$19 .$.

$19 .$.

$19 .$.

$19 .$.

SEP

Q9...

०9...

$09 .$.

$\odot 9$.

$09 .$.

$09 \ldots$

$<0.019$

--

$--$

$\odot .179$

$<0.019$

--

$-$

$<0.019$

$<0.019$

$<0.019$

$\odot .216$

0.034

$\odot .049$

Dis -

solved

Manga- solids

nese, dried@

water, 180degc

fltrd, wat flt

$\mathrm{ug} / \mathrm{L} \quad \mathrm{mg} / \mathrm{L}$

(01056) (70300)

MAY

$\begin{array}{llr}13 \ldots & -- & -- \\ 13 \ldots & <1.00 & 256\end{array}$




\section{GENEVA LAKE AT WEST END NEAR WILLIAMS BAY, WI}

LAKE-DEPTH PROFILES, NOVEMBER 19, 2013 TO JUNE 26, 2014

$11-19-13$
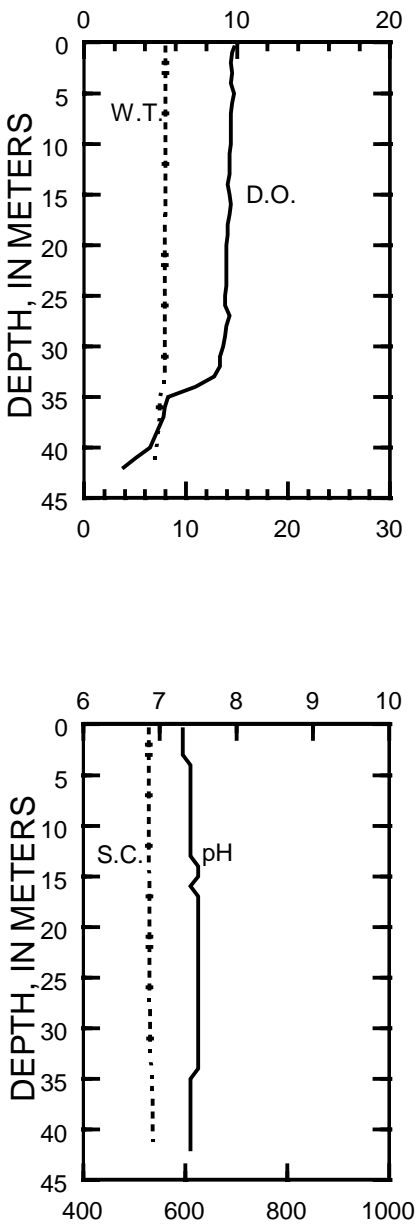

03-04-14

05-13-14

DISSOLVED OXYGEN (D.O.), IN MILLIGRAMS PER LITER
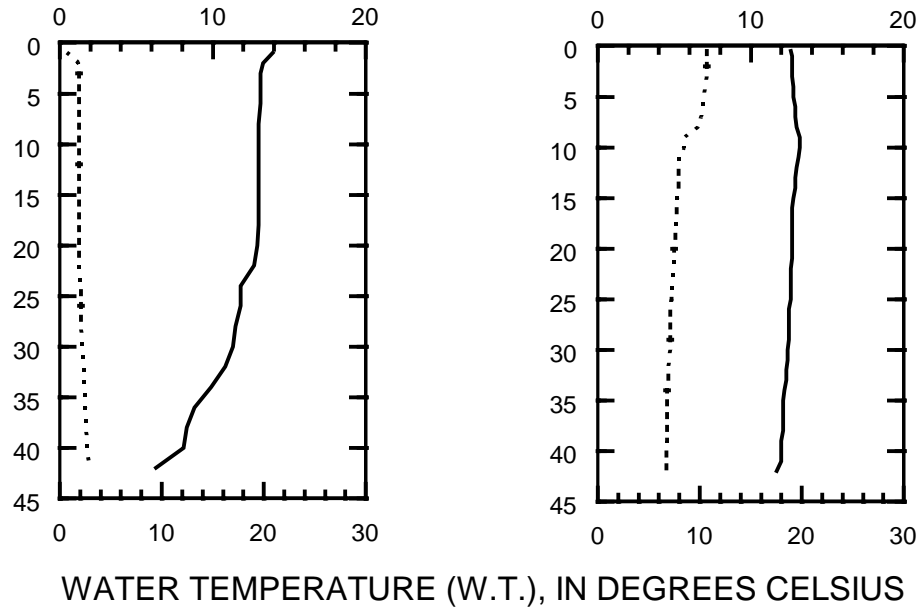

WATER TEMPERATURE (W.T.), IN DEGREES CELSIUS

$\mathrm{PH}$, IN STANDARD UNITS
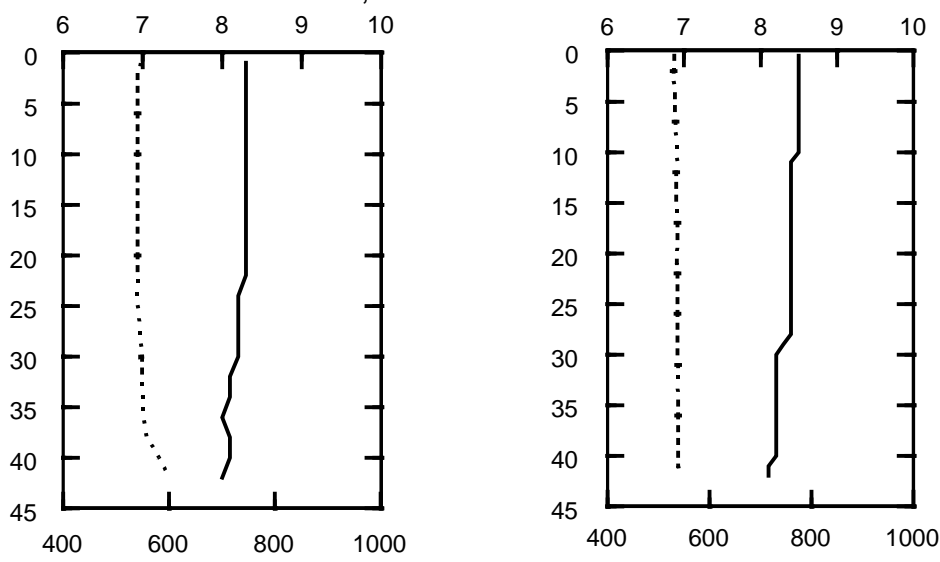

$06-26-14$
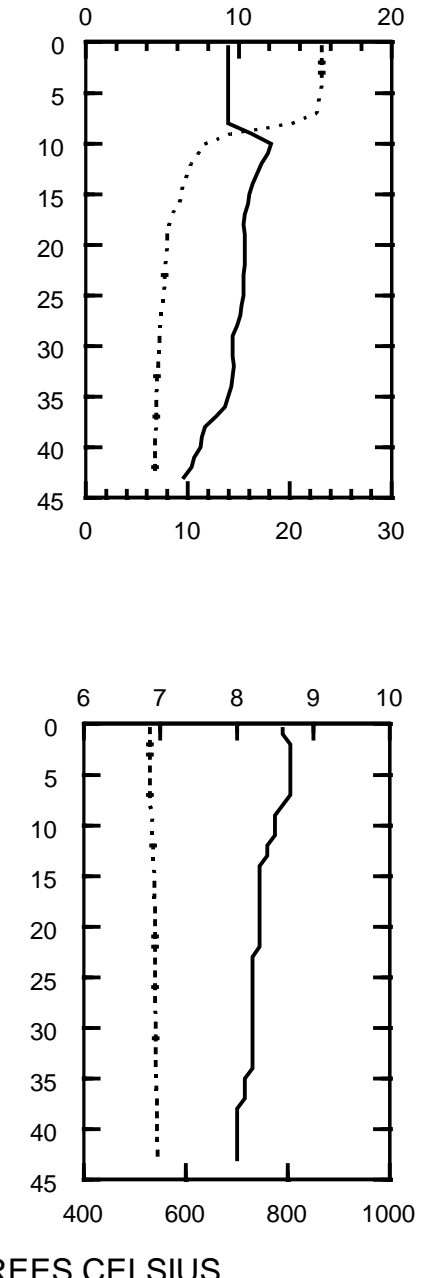
423329088323300 GENEVA LAKE AT WEST END NEAR WILLIAMS BAY, WI

LAKE-DEPTH PROFILES, JULY 29 TO SEPTEMBER 9, 2014

$07-29-14$
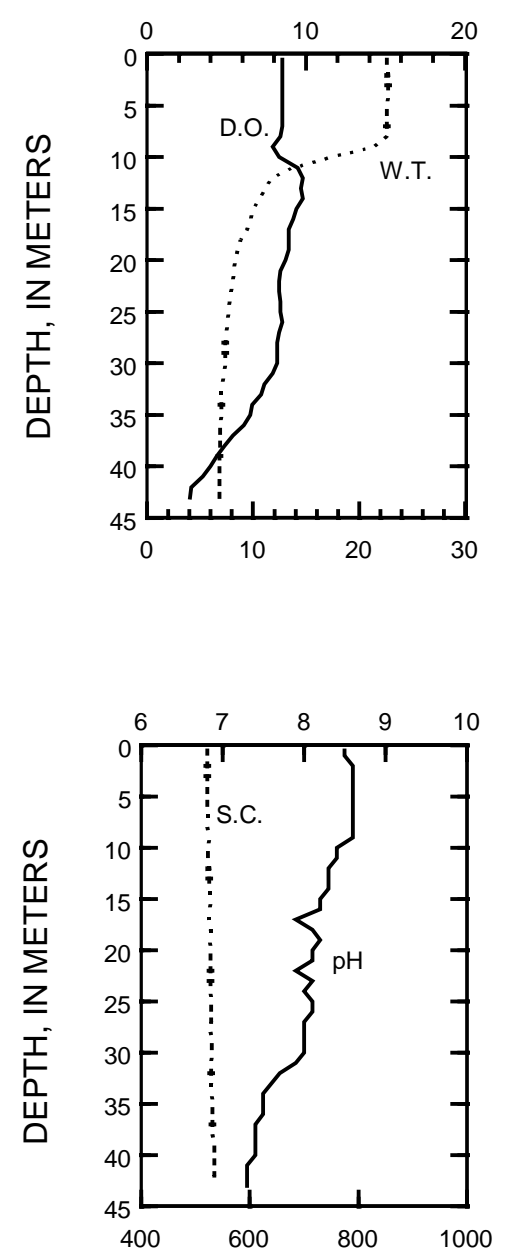

08-19-14

DISSOLVED OXYGEN (D.O.), IN MILLIGRAMS PER LITER

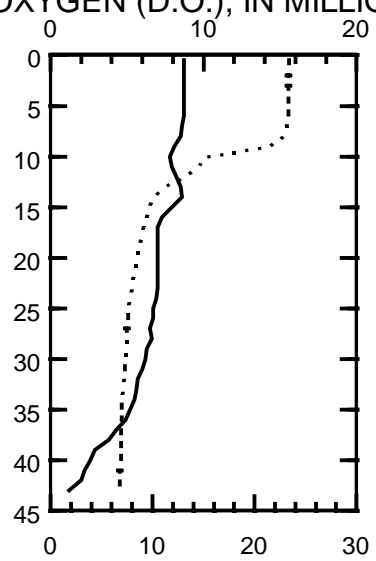

WATER TEMPERATURE (W.T.), IN DEGREES CELSIUS

$\mathrm{PH}$, IN STANDARD UNITS

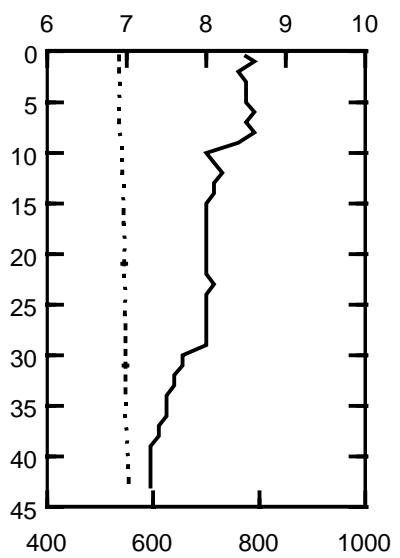

09-09-14
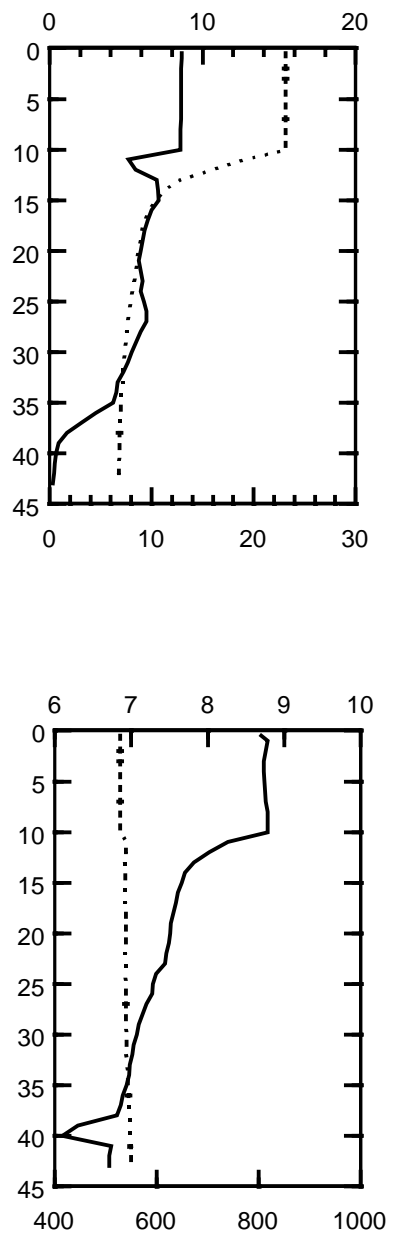

SPECIFIC CONDUCTANCE (S.C.), IN MICROSIEMENS PER CENTIMETER AT 25 DEGREES CELSIUS 

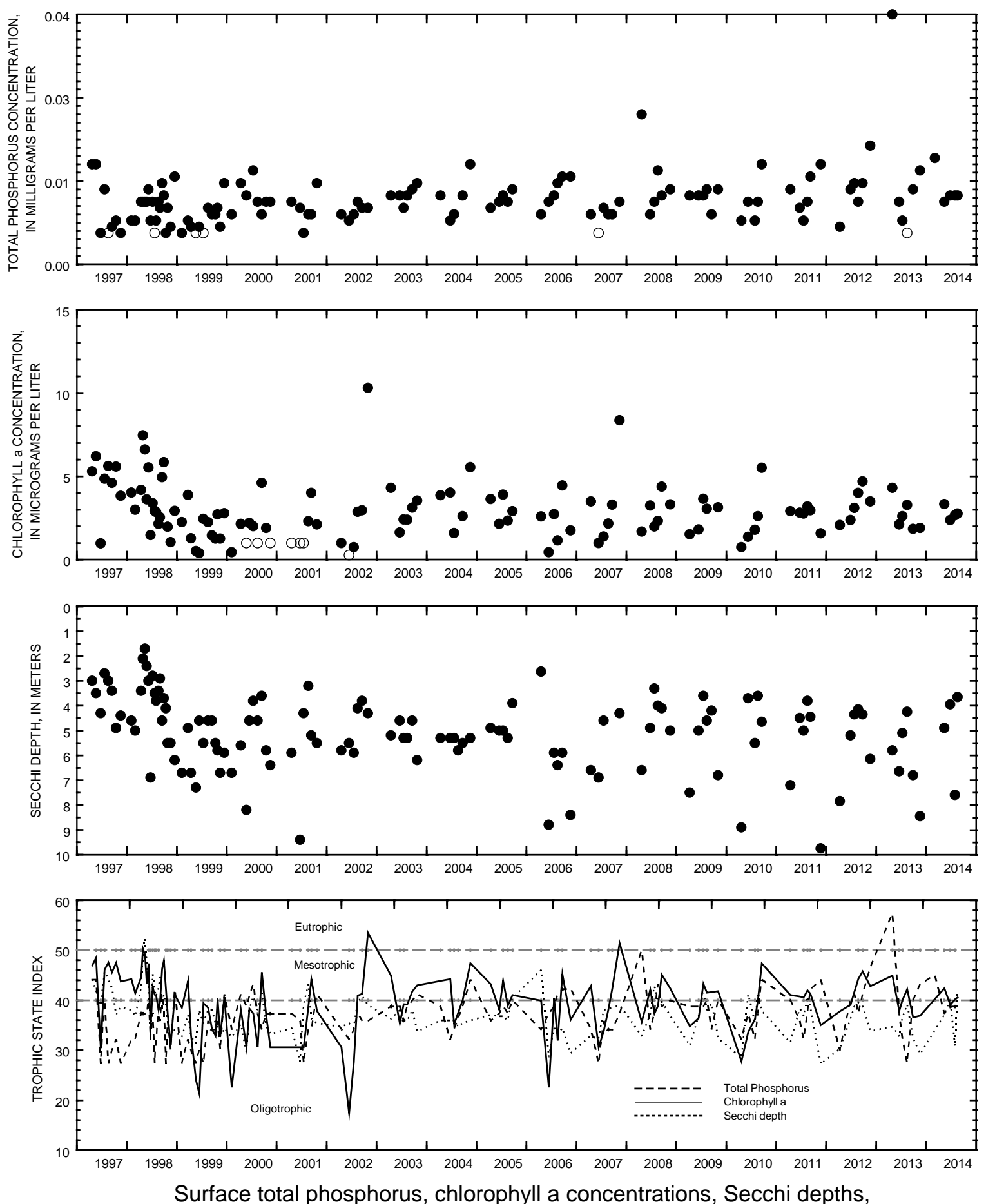
and TSI data for Geneva Lake, West End, near Williams Bay, Wisconsin.

(Open circles on the first two plots indicate laboratory detection limit for selected analyses. 


\section{GREEN LAKE AT GREEN LAKE, WI}

LOCATION.--Lat 4348'40", long 8900'00" referenced to North American Datum of 1983, in SE 1/4 SW 1/4 Sec.31, T.16 N., R.13 E., Green Lake County, WI, Hydrologic Unit 04030201, on left bank at upstream side of County Highway K, 11.6 mi southwest of Green Lake.

SURFACE AREA.--11.48 $\mathrm{mi}^{2}$.

DRAINAGE AREA.--103 mi2; including surface area of Green Lake.

PERIOD OF RECORD.--October 1993 to May 2012, October 2012 to September 2013. Data from October 1993 to May 2012 previously available as 434928088553601 Green Lake at County Trunk HWY A near Green Lake, WI.

GAGE.--Water-stage recorder. Datum of gage is $790.132 \mathrm{ft}$ above NAVD of 1988 (from Wisconsin Department of Transportation Benchmark) COOPERATION.--Operated in cooperation with Green Lake Sanitary District.

REMARKS.--Lake level regulated by dam at outlet at Green Lake. Gage-height telemeter at station.

EXTREMES FOR PERIOD OF RECORD.--Maximum recorded gage height, $8.67 \mathrm{ft}$, June 15, 2008; minimum recorded, $5.27 \mathrm{ft}$, Nov. 5, 2005. EXTREMES FOR CURRENT YEAR.--Maximum recorded gage height, $6.96 \mathrm{ft}$, Jun. 18; minimum recorded gage height, $5.81 \mathrm{ft}$, Apr. 4.

GAGE HEIGHT, FEET

WATER YEAR OCTOBER 2013 TO SEPTEMBER 2014

DAILY MEAN VALUES

\begin{tabular}{|c|c|c|c|c|c|c|c|c|c|c|c|c|}
\hline \multicolumn{13}{|c|}{ [e, estimated] } \\
\hline DAY & OCT & NOV & DEC & JAN & FEB & MAR & APR & MAY & JUN & JUL & AUG & SEP \\
\hline 1 & 6.17 & 6.10 & 6.24 & 6.17 & 6.17 & 6.15 & 6.18 & 6.55 & 6.56 & 6.62 & 6.47 & 6.48 \\
\hline 2 & 6.17 & 6.12 & 6.23 & 6.17 & 6.16 & 6.15 & 6.17 & 6.54 & 6.82 & 6.65 & 6.48 & 6.48 \\
\hline 3 & 6.18 & 6.12 & 6.26 & 6.16 & 6.17 & 6.14 & 6.16 & 6.56 & 6.85 & 6.65 & 6.49 & 6.47 \\
\hline 4 & 6.19 & 6.12 & 6.27 & 6.17 & 6.16 & 6.14 & 6.17 & 6.56 & 6.85 & 6.65 & 6.53 & 6.46 \\
\hline 5 & 6.19 & 6.14 & 6.24 & 6.16 & 6.17 & 6.15 & 6.16 & 6.56 & 6.82 & 6.63 & 6.52 & 6.48 \\
\hline 6 & 6.18 & 6.21 & 6.24 & 6.16 & 6.16 & 6.14 & 6.15 & 6.55 & 6.78 & 6.64 & 6.52 & 6.47 \\
\hline 7 & 6.17 & 6.22 & 6.23 & 6.15 & 6.14 & 6.14 & 6.14 & 6.57 & 6.77 & 6.66 & 6.48 & 6.46 \\
\hline 8 & 6.16 & 6.21 & 6.23 & 6.15 & 6.15 & 6.13 & 6.14 & 6.56 & 6.78 & 6.72 & 6.47 & 6.44 \\
\hline 9 & 6.15 & 6.21 & 6.21 & 6.15 & 6.15 & 6.12 & 6.13 & 6.56 & 6.73 & 6.70 & 6.46 & 6.43 \\
\hline 10 & 6.14 & 6.21 & 6.20 & 6.16 & 6.15 & 6.12 & 6.14 & 6.56 & 6.69 & 6.66 & 6.45 & 6.44 \\
\hline 11 & 6.14 & 6.23 & 6.19 & 6.18 & 6.15 & 6.12 & 6.14 & 6.57 & 6.66 & 6.60 & 6.45 & 6.42 \\
\hline 12 & 6.12 & 6.21 & 6.18 & 6.18 & 6.15 & 6.14 & 6.20 & 6.69 & 6.61 & 6.60 & 6.45 & 6.40 \\
\hline 13 & 6.12 & 6.18 & 6.20 & 6.17 & 6.16 & 6.12 & 6.39 & 6.76 & 6.58 & 6.62 & 6.44 & 6.38 \\
\hline 14 & 6.12 & 6.19 & 6.19 & 6.18 & 6.15 & 6.13 & 6.66 & 6.75 & 6.57 & 6.63 & 6.43 & 6.35 \\
\hline 15 & 6.12 & 6.19 & 6.17 & 6.20 & 6.15 & 6.16 & 6.67 & 6.75 & 6.53 & 6.60 & 6.41 & 6.35 \\
\hline 16 & 6.13 & 6.19 & 6.16 & 6.20 & 6.15 & 6.17 & 6.67 & 6.71 & 6.54 & 6.60 & 6.40 & 6.35 \\
\hline 17 & 6.13 & 6.25 & 6.16 & 6.19 & 6.17 & 6.18 & 6.67 & 6.68 & 6.67 & 6.60 & 6.42 & 6.34 \\
\hline 18 & 6.12 & 6.28 & 6.16 & 6.20 & 6.18 & 6.18 & 6.66 & 6.65 & 6.79 & 6.58 & 6.41 & 6.34 \\
\hline 19 & 6.11 & 6.28 & 6.17 & 6.19 & 6.17 & 6.18 & 6.63 & 6.62 & 6.82 & 6.57 & 6.55 & 6.31 \\
\hline 20 & 6.12 & 6.28 & 6.18 & 6.19 & 6.19 & 6.17 & 6.60 & 6.62 & 6.82 & 6.56 & 6.57 & 6.33 \\
\hline 21 & 6.09 & 6.28 & 6.17 & 6.19 & 6.19 & 6.17 & 6.58 & 6.60 & 6.80 & 6.55 & 6.58 & 6.37 \\
\hline 22 & 6.08 & 6.29 & 6.19 & 6.18 & 6.19 & 6.16 & 6.56 & 6.57 & 6.78 & 6.55 & 6.60 & 6.35 \\
\hline 23 & 6.07 & 6.30 & 6.18 & 6.17 & 6.18 & 6.15 & 6.53 & 6.57 & 6.76 & 6.53 & 6.60 & 6.35 \\
\hline 24 & 6.05 & 6.27 & 6.18 & 6.17 & 6.18 & 6.13 & 6.52 & 6.56 & 6.72 & 6.51 & 6.58 & 6.34 \\
\hline 25 & 6.03 & 6.25 & 6.19 & 6.17 & 6.16 & 6.12 & 6.51 & 6.55 & 6.70 & 6.50 & 6.60 & 6.35 \\
\hline 26 & 6.02 & 6.27 & 6.18 & 6.18 & 6.16 & 6.10 & 6.49 & 6.54 & 6.65 & 6.49 & 6.61 & 6.35 \\
\hline 27 & 6.01 & 6.25 & 6.18 & 6.17 & 6.16 & 6.10 & 6.48 & 6.54 & 6.60 & 6.50 & 6.57 & 6.35 \\
\hline 28 & 6.04 & 6.24 & 6.18 & 6.17 & 6.15 & 6.18 & 6.51 & 6.55 & 6.56 & 6.50 & 6.53 & 6.34 \\
\hline 29 & 6.01 & 6.23 & 6.18 & 6.17 & --- & 6.19 & 6.56 & 6.56 & 6.56 & 6.48 & 6.52 & 6.36 \\
\hline 30 & 6.00 & 6.23 & 6.17 & 6.17 & --- & 6.19 & 6.56 & 6.55 & 6.60 & 6.50 & 6.50 & 6.35 \\
\hline 31 & 6.07 & --- & 6.18 & 6.17 & -- & 6.19 & -- & 6.53 & -- & 6.49 & 6.49 & --- \\
\hline MEAN & 6.11 & 6.22 & 6.20 & 6.17 & 6.16 & 6.15 & 6.40 & 6.60 & 6.70 & 6.59 & 6.50 & 6.39 \\
\hline MAX & 6.19 & 6.30 & 6.27 & 6.20 & 6.19 & 6.19 & 6.67 & 6.76 & 6.85 & 6.72 & 6.61 & 6.48 \\
\hline MIN & 6.00 & 6.10 & 6.16 & 6.15 & 6.14 & 6.10 & 6.13 & 6.53 & 6.53 & 6.48 & 6.40 & 6.31 \\
\hline
\end{tabular}




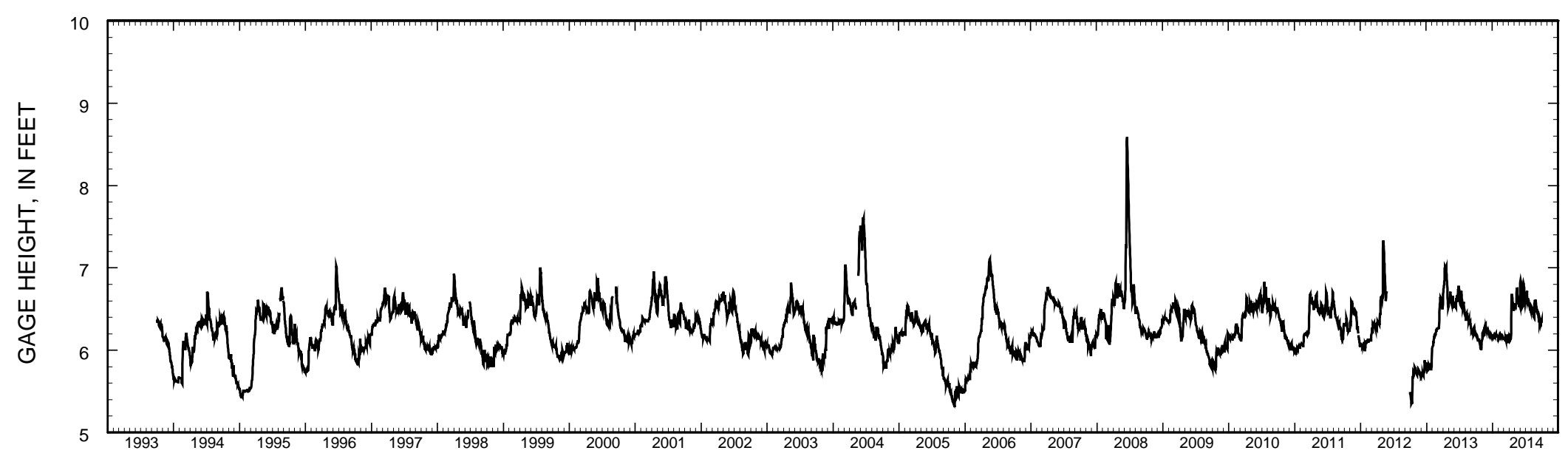

Stage hydrograph for Green Lake, 1993-2014. 
434756089020500 GREEN LAKE AT DEEP HOLE NEAR GREEN LAKE, WI

LOCATION.--Lat $43^{\circ} 47^{\prime} 56^{\prime \prime}$, long $89^{\circ} 02^{\prime} 05^{\prime \prime}$, in NW 1/4 SE 1/4 sec.2, T.15 N., R.12 E., Green Lake County, Hydrologic Unit 04030201, about 5 miles southwest of the City of Green Lake.

SURFACE AREA.--11.48 $\mathrm{mi}^{2}$.

PERIOD OF RECORD.--May 2004 to current year. Lake sampled by Wisconsin Department of Natural Resources prior to 2004.

REMARKS.--Water-quality analyses done by Wisconsin State Laboratory of Hygiene. A "*” indicates data that were collected by Mary Jane Bumby, Citizen Lake Monitoring Volunteer. WATER-QUALITY DATA, MARCH 5 TO SEPTEMBER 25, 2014

(Milligrams per liter unless otherwise indicated)

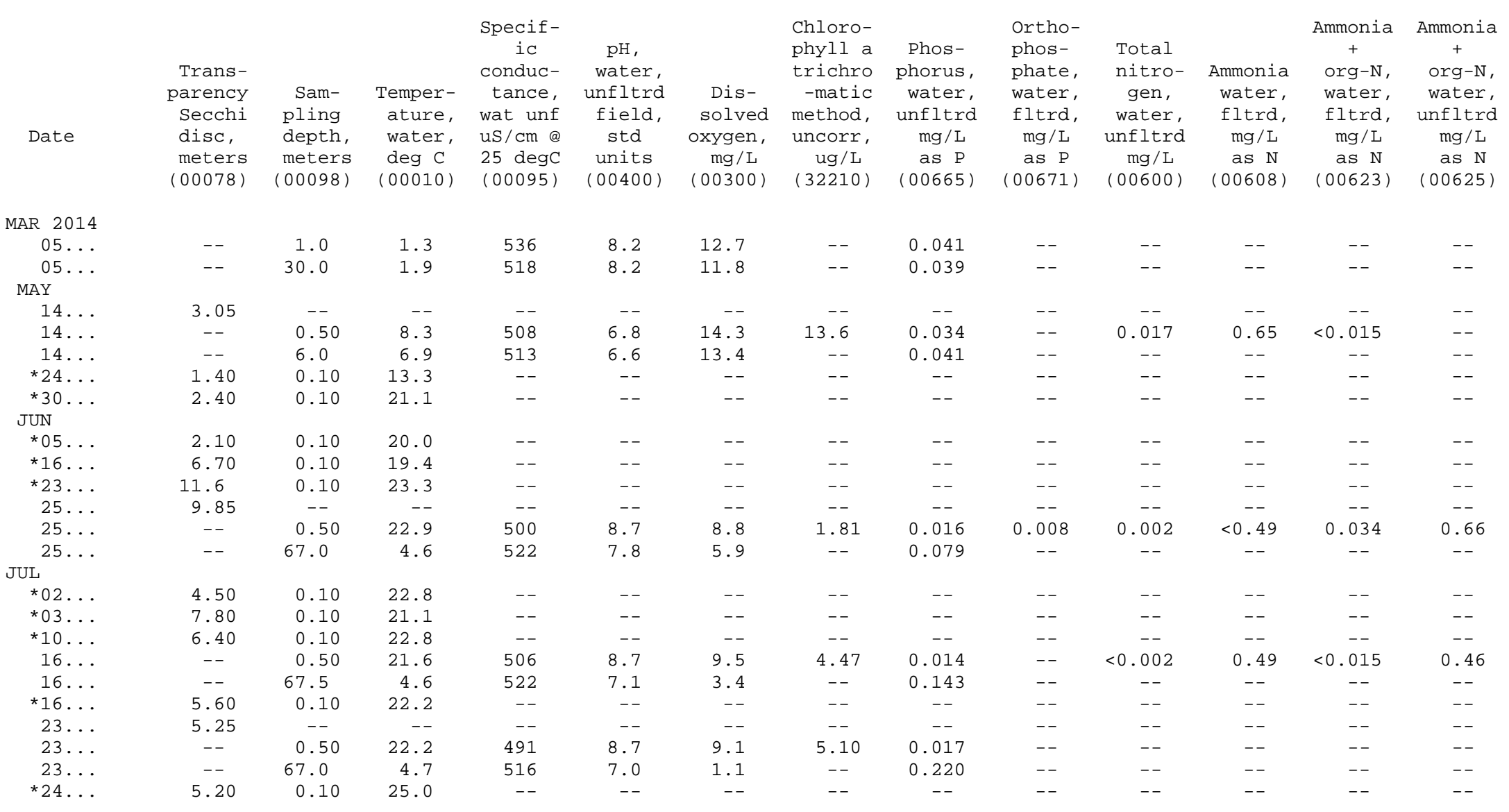


434756089020500 GREEN LAKE AT DEEP HOLE NEAR GREEN LAKE, WI

WATER-QUALITY DATA, MARCH 5 TO SEPTEMBER 25, 2014

(Milligrams per liter unless otherwise indicated)

\begin{tabular}{|c|c|c|c|c|c|c|c|c|c|c|c|c|c|}
\hline Date & $\begin{array}{c}\text { Trans- } \\
\text { parency } \\
\text { Secchi } \\
\text { disc, } \\
\text { meters } \\
(00078)\end{array}$ & $\begin{array}{c}\text { Sam- } \\
\text { pling } \\
\text { depth, } \\
\text { meters } \\
(\odot \odot \odot 98)\end{array}$ & $\begin{array}{c}\text { Temper- } \\
\text { ature, } \\
\text { water, } \\
\text { deg C } \\
(\odot \odot 010)\end{array}$ & $\begin{array}{c}\text { Specif- } \\
\text { ic } \\
\text { conduc- } \\
\text { tance, } \\
\text { wat unf } \\
\text { us/cm @ } \\
25 \text { degc } \\
(00095)\end{array}$ & $\begin{array}{c}\text { pH, } \\
\text { water, } \\
\text { unfltrd } \\
\text { field, } \\
\text { std } \\
\text { units } \\
(0 \odot 40 \odot)\end{array}$ & $\begin{array}{c}\text { Dis- } \\
\text { solved } \\
\text { oxygen, } \\
\text { mg/L } \\
(\odot \odot 30 \odot)\end{array}$ & $\begin{array}{c}\text { Chloro- } \\
\text { phyll a } \\
\text { trichro } \\
\text {-matic } \\
\text { method, } \\
\text { uncorr, } \\
\text { ug/L } \\
(32210)\end{array}$ & $\begin{array}{c}\text { Phos- } \\
\text { phorus, } \\
\text { water, } \\
\text { unfltrd } \\
\text { mg/L } \\
\text { as P } \\
(00665)\end{array}$ & $\begin{array}{l}\text { Ortho- } \\
\text { phos- } \\
\text { phate, } \\
\text { water, } \\
\text { fltrd, } \\
\text { mg/L } \\
\text { as P } \\
(0 \odot 671)\end{array}$ & $\begin{array}{c}\text { Total } \\
\text { nitro- } \\
\text { gen, } \\
\text { water, } \\
\text { unfltrd } \\
\text { mg/L } \\
(006 \odot \odot)\end{array}$ & $\begin{array}{c}\text { Ammonia } \\
\text { water, } \\
\text { fltrd, } \\
\text { mg/L } \\
\text { as N } \\
(00608)\end{array}$ & $\begin{array}{c}\text { Ammonia } \\
+ \\
\text { org-N, } \\
\text { water, } \\
\text { fltrd, } \\
\text { mg/L } \\
\text { as N } \\
(00623)\end{array}$ & $\begin{array}{c}\text { Ammonia } \\
+ \\
\text { org-N, } \\
\text { water, } \\
\text { unfltrd } \\
\text { mg/L } \\
\text { as N } \\
(00625)\end{array}$ \\
\hline \multicolumn{14}{|l|}{ AUG } \\
\hline *06. . & 5.30 & $\odot .10$ & 26.1 & - - & -- & - - & - - & -- & - - & - - & - - & - - & - - \\
\hline $12 \ldots$ & 4.45 & -- & - - & - - & - - & - - & - - & - - & - - & - - & - - & - - & - - \\
\hline $12 \ldots$ & -- & 0.50 & 23.7 & 501 & 8.7 & 9.2 & 6.67 & $\odot .016$ & - - & $<0.002$ & $<0.67$ & $<0.015$ & 0.51 \\
\hline $12 \ldots$ & -- & 67.0 & 4.6 & 533 & 6.4 & 0.6 & - - & 0.164 & - - & - - & -- & - - & - - \\
\hline${ }^{*} 14 \ldots$ & 4.30 & 0.10 & 24.4 & - - & - - & - - & - - & -- & - - & - - & - - & - - & - - \\
\hline $20 \ldots$ & 5.35 & -- & -- & -- & -- & - - & - - & - - & - - & - - & - - & - - & - - \\
\hline $20 \ldots$ & - - & $\odot .5 \odot$ & 23.4 & 500 & 8.4 & 8.8 & 4.30 & $\odot .012$ & -- & -- & -- & - - & -- \\
\hline $20 \ldots$ & -- & 67.5 & 4.6 & 540 & 6.8 & 0.8 & -- & 0.248 & -- & - - & - - & - - & - - \\
\hline${ }^{*} 26 \ldots$ & 4.00 & 0.10 & 23.9 & -- & -- & -- & -- & -- & - - & - - & -- & - - & - - \\
\hline \multicolumn{14}{|l|}{ SEP } \\
\hline${ }^{\star} 07 \ldots$ & 5.00 & 0.10 & 24.4 & -- & - - & - - & - - & - - & - - & - - & - - & - - & - - \\
\hline $23 \ldots$ & 5.85 & -- & - - & - & -- & - - & -- & -- & - - & -- & -- & - - & - \\
\hline $23 \ldots$ & -- & 0.50 & 17.9 & 497 & 8.7 & 9.5 & 7.84 & 0.016 & -- & 0.003 & $<0.55$ & $<0.015$ & 0.60 \\
\hline $23 \ldots$ & - - & 14.0 & 9.5 & 516 & 8.0 & 6.6 & - - & $\odot .012$ & - - & - - & -- & - - & -- \\
\hline $23 \ldots$ & -- & $5 \odot . \odot$ & 4.8 & 518 & 7.9 & 9.3 & -- & 0.055 & -- & -- & - - & - - & - \\
\hline $23 \ldots$ & - - & 63.0 & 4.7 & 522 & 7.6 & 3.5 & - - & 0.128 & - - & - - & - - & - - & -- \\
\hline $23 \ldots$ & - - & 66.0 & 4.6 & 531 & 7.5 & 0.2 & - - & 0.161 & - - & - - & -- & - - & - \\
\hline $23 \ldots$ & - - & 67.0 & 4.6 & 533 & 7.5 & 0.1 & - - & 0.268 & - - & - - & - - & - - & - - \\
\hline${ }^{*} 25 \ldots$ & 5.50 & 0.10 & 18.9 & -- & -- & - & - - & -- & -- & - - & - - & - - & - - \\
\hline
\end{tabular}


434756089020500 GREEN LAKE AT DEEP HOLE NEAR GREEN LAKE, WI

WATER-QUALITY DATA, MARCH 5 TO SEPTEMBER 25, 2014

(Milligrams per liter unless otherwise indicated)

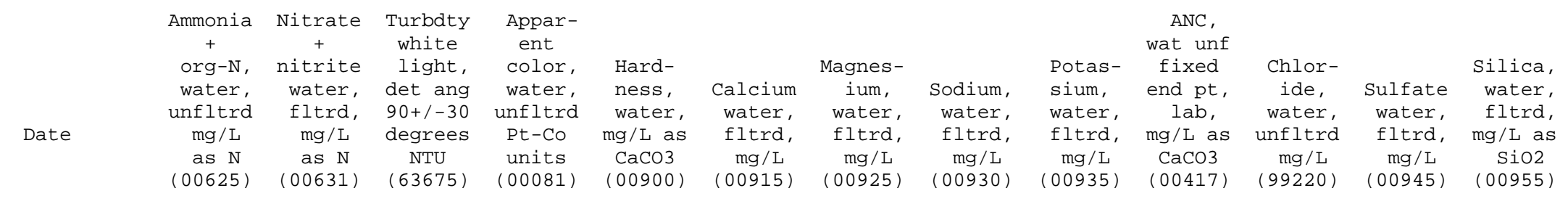

MAY

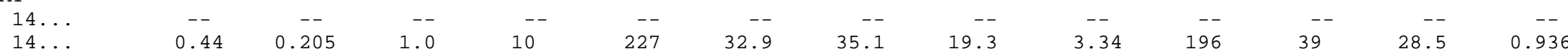

$14 \ldots$

$0.44-0.205 \quad 1.0$

10

. 1

UN

$25 \ldots$

$25 \ldots$

JUL

$16 \ldots$

$16 \ldots$

AUG

$12 \ldots$

$12 .$.

SEP

$-$

$0.48<0.019$

$0.46 \quad 0.028$

- $\quad--$

$0 . \overline{6}<<<-\overline{019}$

- - - -

$23 \ldots$

$23 \ldots$

$23 \ldots$

23.

23.

$23 .$.

$0.53<0.019$

$--\quad-$

$\begin{array}{ll}- & - \\ -- & -\end{array}$

$--\quad \quad-$

$\begin{array}{cccc} & \text { Mron, } & \text { Manga- } & \begin{array}{c}\text { nese, } \\ \text { solids }\end{array} \\ \text { water, } & \text { water, } & 180 \mathrm{deg} \\ \text { Date } & \mathrm{fltrd}, & \mathrm{fltrd}, & \text { wat } \mathrm{flt} \\ \mathrm{ug} / \mathrm{L} & \mathrm{ug} / \mathrm{L} & \mathrm{mg} / \mathrm{L} \\ (01046) & (01056) & (70300)\end{array}$

MAY

$14 \ldots$

$14 \ldots$

$<0.100$

$<1.00$

264

$14 \ldots$

- -

--
--
--

$-\frac{1}{2}$

3.34
--
--
--
--
--
--
--
-
-
-
-
--
--
-
-
--
--

--
--
--
--
--
--
--
--
--
--
--
--
--
--
--
--
--

$-$

- -

๑. 936

$--$

$--$

$-$

-

$\begin{array}{lll}-- & -1 & - \\ -- & - & -\end{array}$

$-$

- -

- 
434756089020500 GREEN LAKE AT DEEP HOLE NEAR GREEN LAKE, WI

LAKE-DEPTH PROFILES, MARCH 5 TO JULY 16, 2014

03-05-14
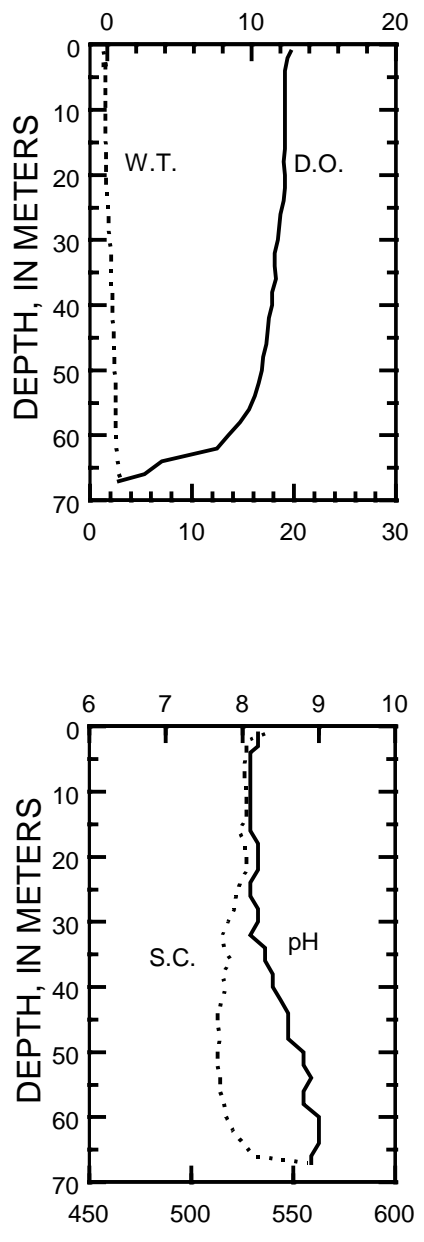

05-14-14

06-25-14

DISSOLVED OXYGEN (D.O.), IN MILLIGRAMS PER LITER
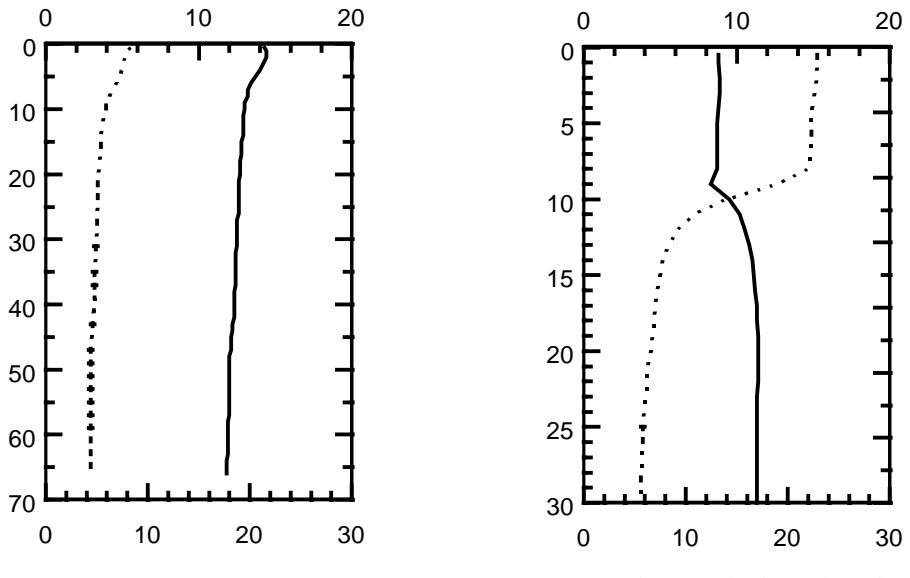

WATER TEMPERATURE (W.T.), IN DEGREES CELSIUS

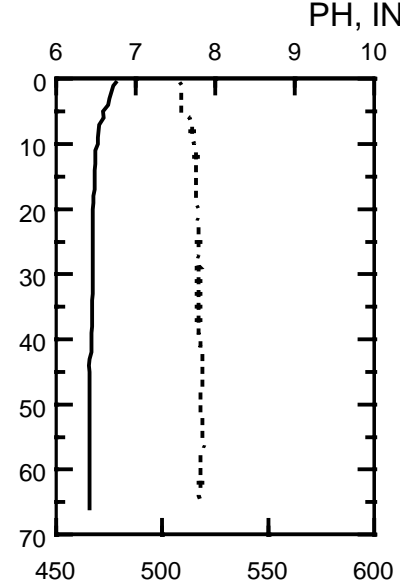

07-16-14
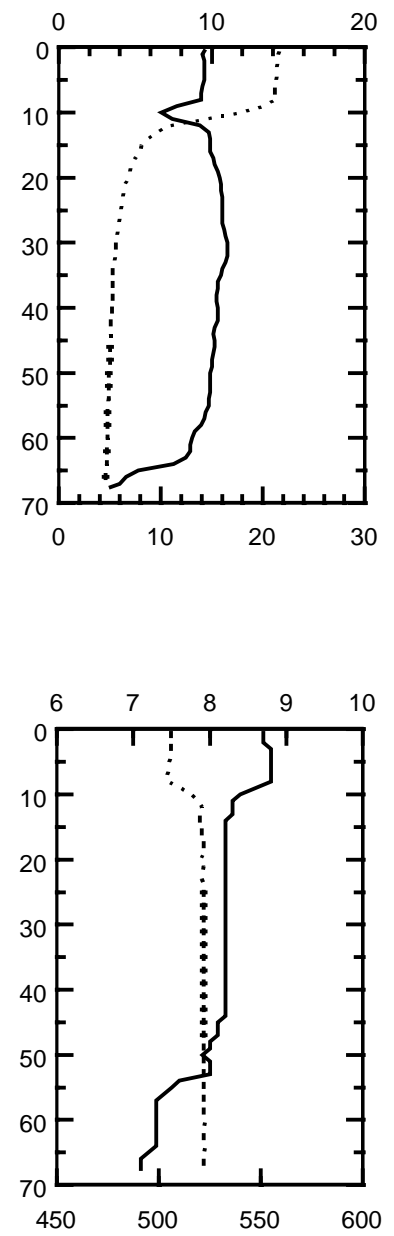

SPECIFIC CONDUCTANCE (S.C.), IN MICROSIEMENS PER CENTIMETER AT 25 DEGREES CELSIUS 


\section{GREEN LAKE AT DEEP HOLE NEAR GREEN LAKE, WI}

LAKE-DEPTH PROFILES, JULY 23 TO SEPTEMBER 23, 2014

07-23-14
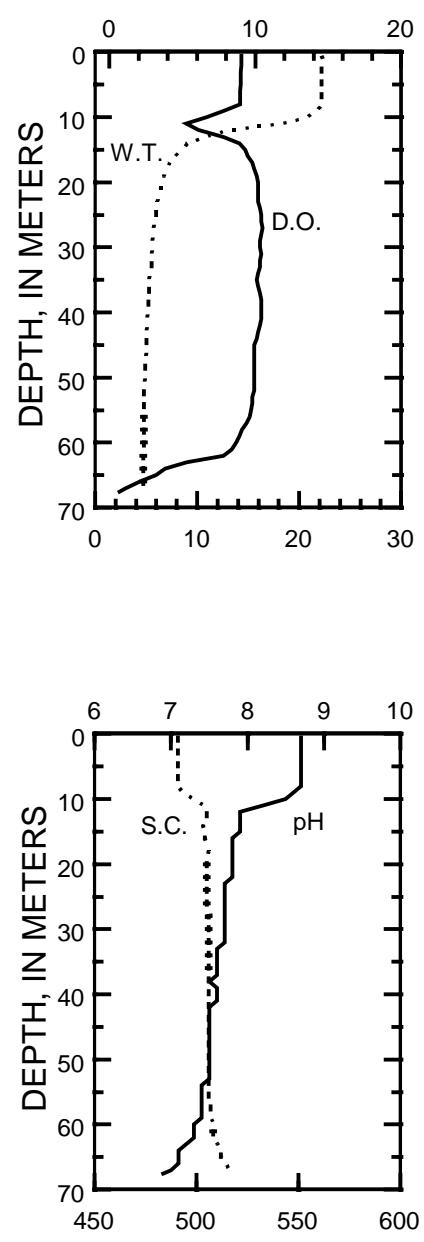

08-12-14

DISSOLVED OXYGEN (D.O.), IN MILLIGRAMS PER LITER
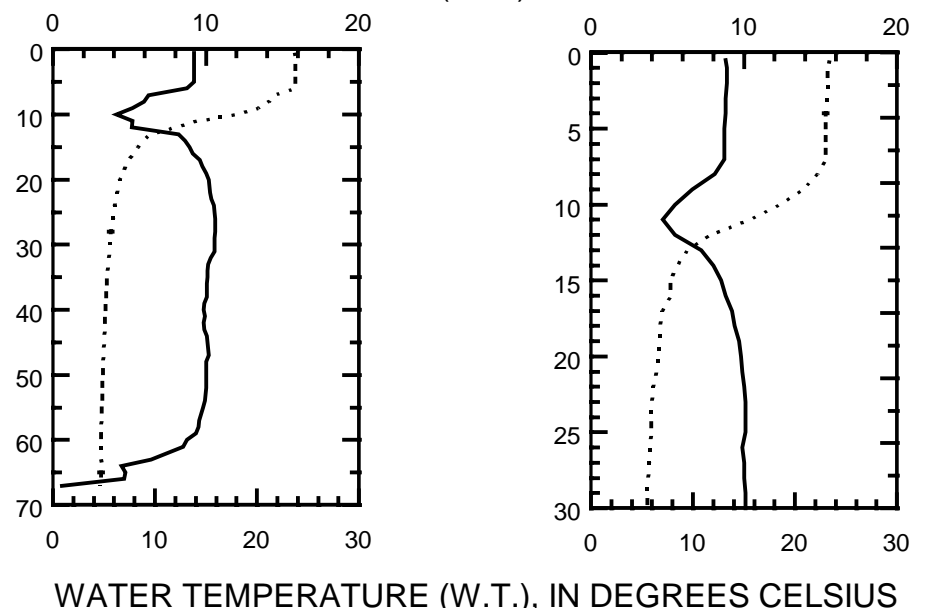

PH, IN STANDARD UNITS

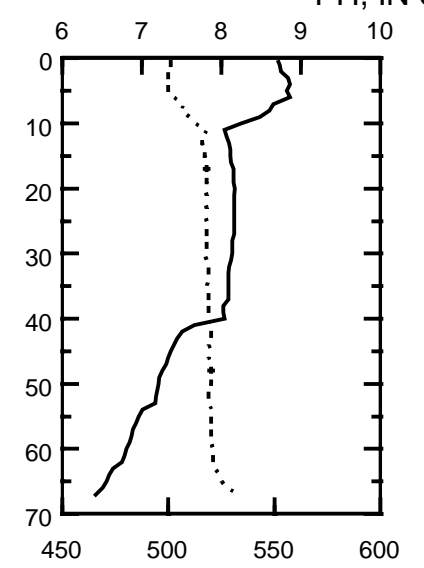

$09-23-14$
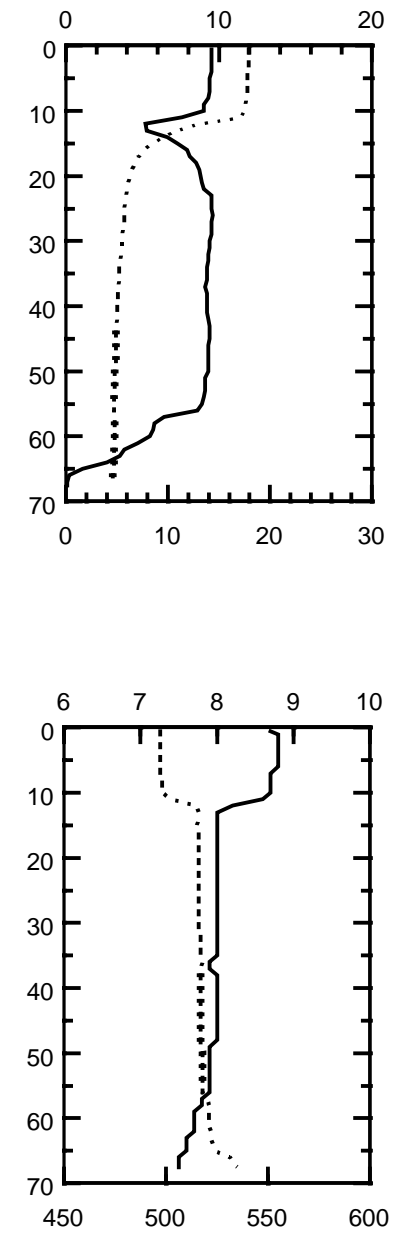

SPECIFIC CONDUCTANCE (S.C.), IN MICROSIEMENS PER CENTIMETER AT 25 DEGREES CELSIUS 

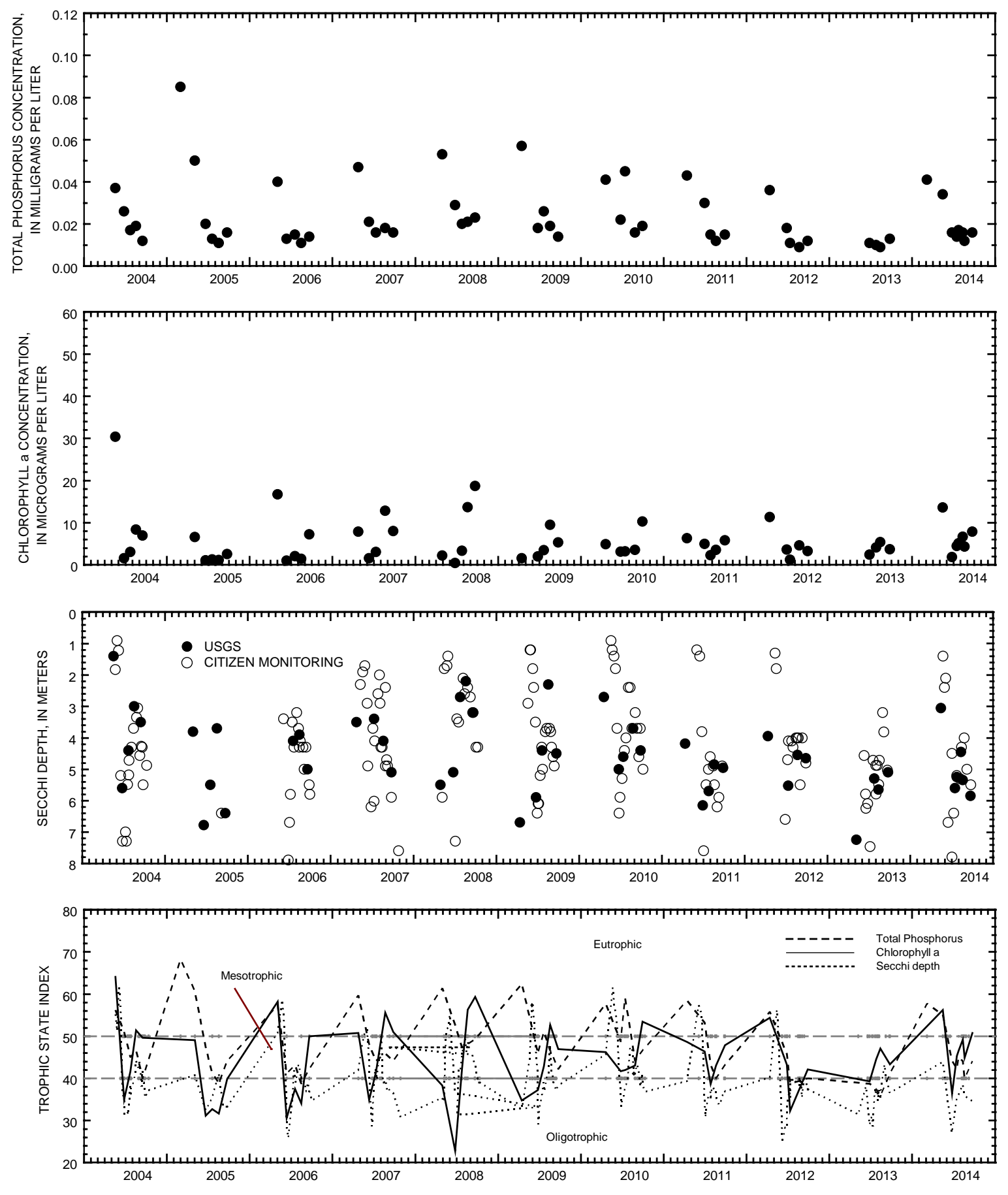

Surface total phosphorus, chlorophyll a concentrations, Secchi depths, and TSI data for Green Lake, Deep Hole, near Green Lake, Wisconsin. 


\section{GREEN LAKE AT EAST END NEAR GREEN LAKE, WI}

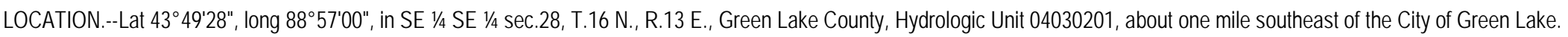
SURFACE AREA.--11.48 mi²

PERIOD OF RECORD.--May 2004 to current year. Lake sampled by Wisconsin Department of Natural Resources prior to 2004.

REMARKS.--Water-quality analyses done by Wisconsin State Laboratory of Hygiene. A "*” indicates data that were collected by Mary Jane Bumby, Citizen Lake Monitoring Volunteer.

\begin{tabular}{|c|c|c|c|c|c|c|c|c|}
\hline Date & $\begin{array}{c}\text { Trans- } \\
\text { parency } \\
\text { Secchi } \\
\text { disc, } \\
\text { meters } \\
(\odot \odot \odot 78)\end{array}$ & $\begin{array}{c}\text { Sam- } \\
\text { pling } \\
\text { depth, } \\
\text { meters } \\
(\odot \odot \odot 98)\end{array}$ & $\begin{array}{c}\text { Temper - } \\
\text { ature, } \\
\text { water, } \\
\text { deg C } \\
(0 \odot 010)\end{array}$ & $\begin{array}{c}\text { Specif- } \\
\text { ic } \\
\text { conduc- } \\
\text { tance, } \\
\text { wat unf } \\
\text { uS/cm @ } \\
25 \text { degC } \\
(0 \odot \odot 95)\end{array}$ & $\begin{array}{c}\text { pH, } \\
\text { water, } \\
\text { unfltrd } \\
\text { field, } \\
\text { std } \\
\text { units } \\
(0 \odot 4 \odot \odot)\end{array}$ & $\begin{array}{c}\text { Dis- } \\
\text { solved } \\
\text { oxygen, } \\
\mathrm{mg} / \mathrm{L} \\
(0030 \odot)\end{array}$ & $\begin{array}{l}\text { Chloro- } \\
\text { phyll a } \\
\text { trichro } \\
\text {-matic } \\
\text { method, } \\
\text { uncorr, } \\
\text { ug/L } \\
(32210)\end{array}$ & $\begin{array}{c}\text { Phos- } \\
\text { phorus, } \\
\text { water, } \\
\text { unfltrd } \\
\mathrm{mg} / \mathrm{L} \\
\text { as } \mathrm{P} \\
(00665)\end{array}$ \\
\hline \multicolumn{9}{|c|}{ MAY 2014} \\
\hline $14 \ldots$ & 3.05 & - - & - - & - - & - - & - - & - - & - - \\
\hline $14 \ldots$ & -- & 0.50 & 8.0 & 513 & 6.7 & 13.9 & 13.8 & 0.036 \\
\hline $14 \ldots$ & - - & 31.0 & 6.4 & 515 & 6.5 & 12.6 & - - & 0.083 \\
\hline$* 24 \ldots$ & 1.50 & 0.10 & 16.1 & - - & -- & - - & - - & - - \\
\hline * $30 \ldots$ & 2.40 & 0.10 & 20.0 & - - & - - & - - & - - & - - \\
\hline \multicolumn{9}{|l|}{ JUN } \\
\hline${ }^{*} 05 \ldots$ & $2.0 \odot$ & 0.10 & 19.4 & -- & -- & -- & -- & -- \\
\hline${ }^{*} 16 \ldots$ & 8.20 & 0.10 & 20.0 & - - & - - & - - & - - & -- \\
\hline *23... & 11.6 & 0.10 & 22.8 & - - & -- & -- & - - & - - \\
\hline $25 \ldots$ & 8.30 & -- & -- & -- & -- & -- & -- & -- \\
\hline $25 \ldots$ & -- & 0.50 & 22.0 & 508 & 8.7 & 9.2 & 1.97 & 0.027 \\
\hline $25 \ldots$ & -- & 30.0 & 5.8 & 520 & 8.0 & 9.4 & - - & 0.066 \\
\hline \multicolumn{9}{|l|}{ JUL } \\
\hline${ }^{*} 02 \ldots$ & 4.50 & 0.10 & 22.2 & - - & - - & -- & -- & -- \\
\hline${ }^{*} 03 \ldots$ & 6.70 & 0.10 & 22.2 & - - & - - & - - & - - & - - \\
\hline *10 . . & 5.50 & 0.10 & 23.3 & - - & -- & - - & - - & - - \\
\hline${ }^{*} 16 \ldots$ & 5.20 & 0.10 & 22.2 & - - & - - & - - & - - & - - \\
\hline $16 \ldots$ & -- & 0.50 & 22.6 & 519 & 8.8 & 9.5 & 10.4 & 0.018 \\
\hline $16 \ldots$ & -- & 30.0 & 5.6 & 527 & 8.1 & 9.9 & - - & 0.051 \\
\hline $23 \ldots$ & 4.65 & -- & -- & - - & - - & -- & - - & -- \\
\hline $23 \ldots$ & - - & 0.50 & 23.1 & 493 & 8.7 & 8.9 & 15.4 & 0.029 \\
\hline $23 \ldots$ & -- & 30.0 & 5.7 & 508 & 7.6 & 9.1 & - - & 0.056 \\
\hline$* 24 \ldots$ & 3.40 & 0.10 & 25.0 & - - & -- & - - & - - & - - \\
\hline
\end{tabular}


434928088570000 GREEN LAKE AT EAST END NEAR GREEN LAKE, WI

WATER-QUALITY DATA, MAY 14 TO SEPTEMBER 25, 2014

(Milligrams per liter unless otherwise indicated)

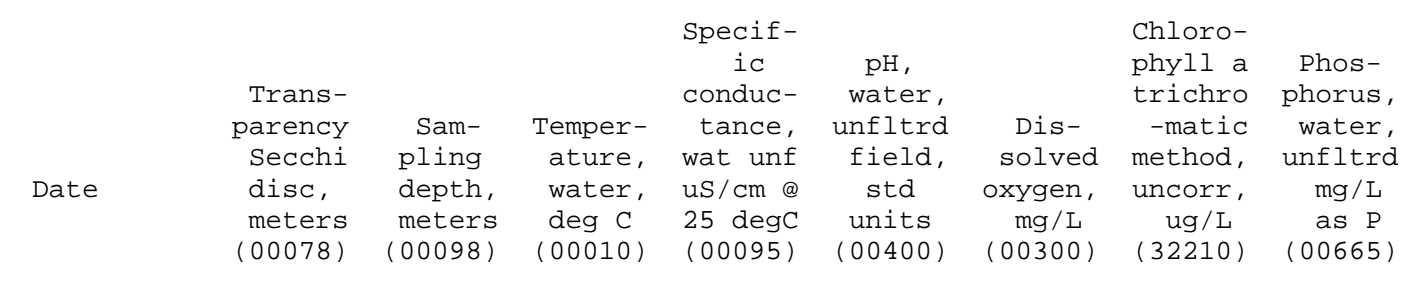

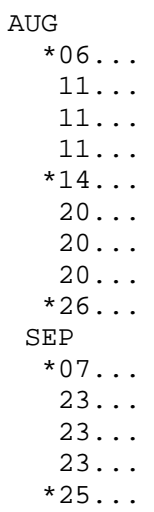

$\begin{array}{ccr}4.30 & 0.10 & 26.7 \\ 5.35 & -- & -- \\ -- & 0.50 & 25.5 \\ -- & 30.0 & 5.6 \\ 5.20 & 0.10 & 24.4 \\ 5.35 & -- & -- \\ -- & 0.50 & 23.1 \\ -- & 30.0 & 5.7 \\ 4.90 & 0.10 & 24.4 \\ & & \\ 3.70 & 0.10 & 25.0 \\ 5.10 & -- & -- \\ -- & 0.50 & 18.6 \\ -- & 31.0 & 5.7 \\ 4.90 & 0.10 & 20.0\end{array}$

$\begin{array}{rrr}-- & -- & -- \\ -- & -- & -- \\ 503 & 8.8 & 9.8 \\ 523 & 8.0 & 8.6 \\ -- & -- & -- \\ -- & -- & -- \\ 502 & 8.9 & 8.9 \\ 522 & 7.9 & 8.5 \\ -- & -- & -- \\ -- & -- & -- \\ -- & -- & - \\ 490 & 8.7 & 9.8 \\ 518 & 7.9 & 4.3 \\ -- & -- & --\end{array}$

$\begin{array}{cc}-- & -- \\ -- & -- \\ 2.44 & 0.015 \\ -- & 0.077 \\ -- & -- \\ -- & -- \\ 4.42 & 0.015 \\ -- & 0.071 \\ -- & -- \\ & \\ -- & -- \\ -- & -- \\ 6.58 & 0.021 \\ -- & 0.101 \\ -- & --\end{array}$


434928088570000 GREEN LAKE AT EAST END NEAR GREEN LAKE, WI

LAKE-DEPTH PROFILES, MAY 14 TO JULY 23, 2014

05-14-14
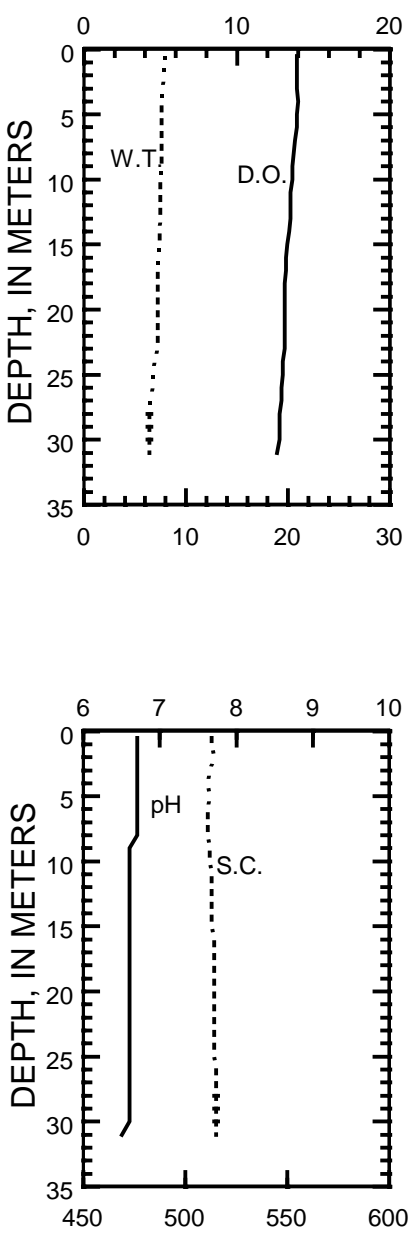

06-25-14

DISSOLVED OXYGEN (D.O), IN MILLIGRAMS PER LITER

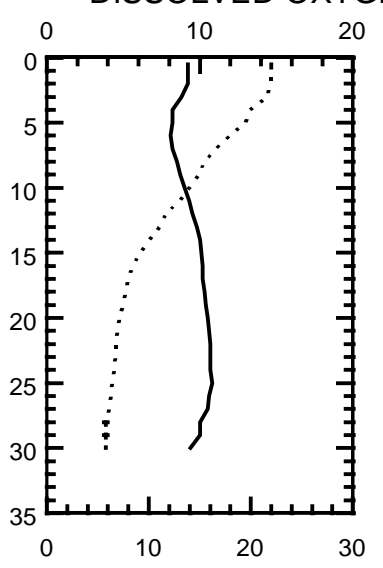

WATER TEMPERATURE (W.T.), IN DEGREES CELSIUS

$\mathrm{PH}$, IN STANDARD UNITS
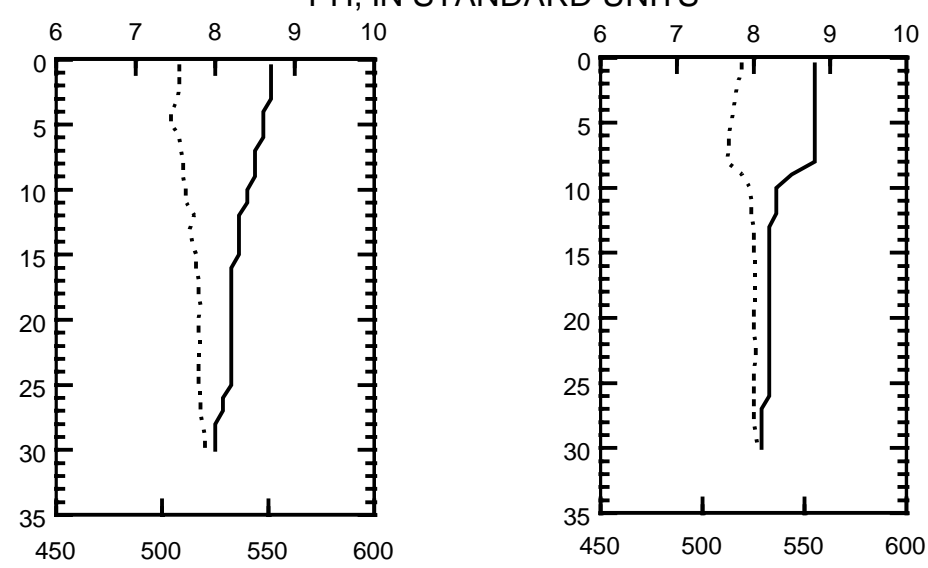

07-23-14
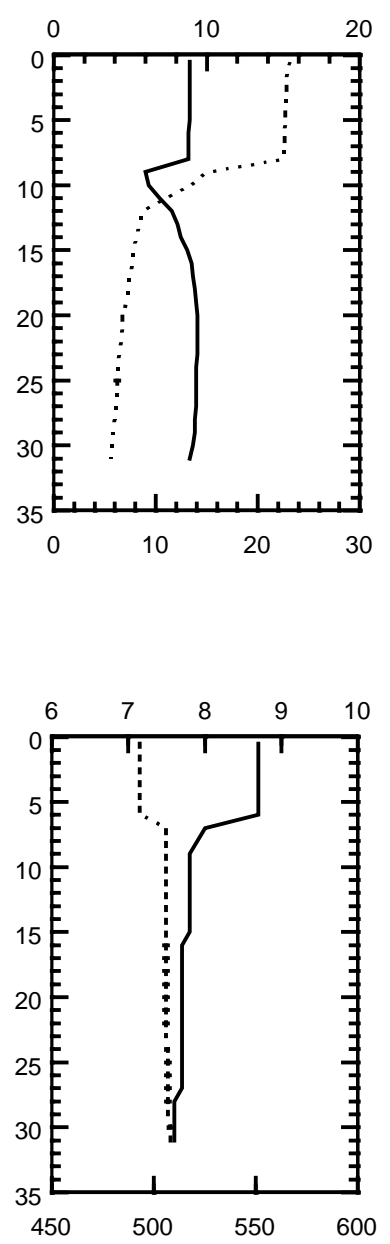

SPECIFIC CONDUCTANCE (S.C.), IN MICROSIEMENS PER CENTIMETER AT 25 DEGREES CELSIUS 
434928088570000 GREEN LAKE AT EAST END NEAR GREEN LAKE, WI

LAKE-DEPTH PROFILES, AUGUST 11 TO SEPTEMBER 9, 2014

08-11-14
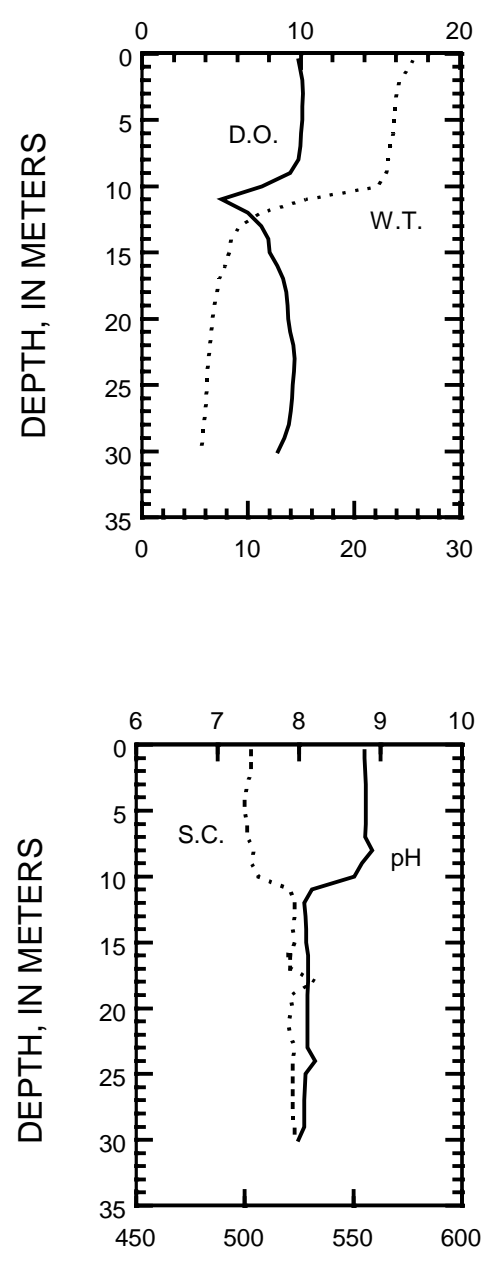

08-20-14

DISSOLVED OXYGEN (D.O.), IN MILLIGRAMS PER LITER

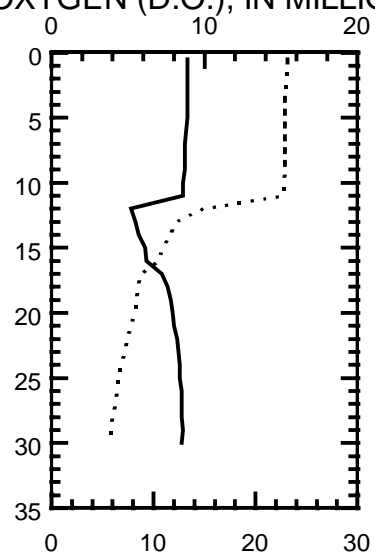

WATER TEMPERATURE (W.T.), IN DEGREES CELSIUS

$\mathrm{PH}$, IN STANDARD UNITS

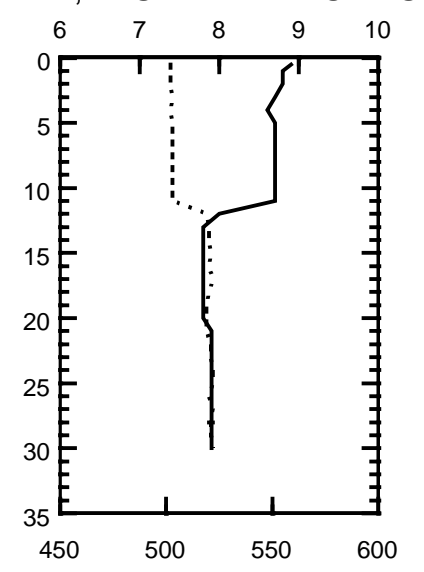

$09-23-14$
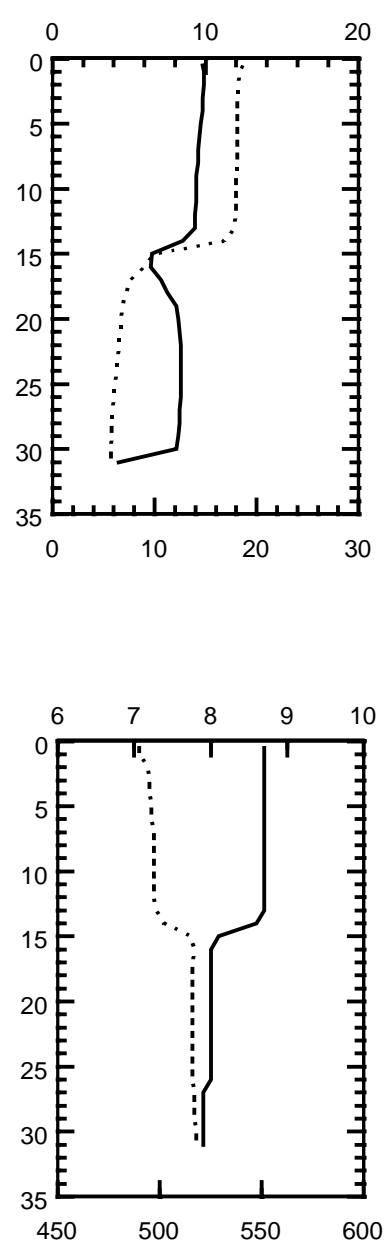

SPECIFIC CONDUCTANCE (S.C.), IN MICROSIEMENS PER CENTIMETER AT 25 DEGREES CELSIUS 

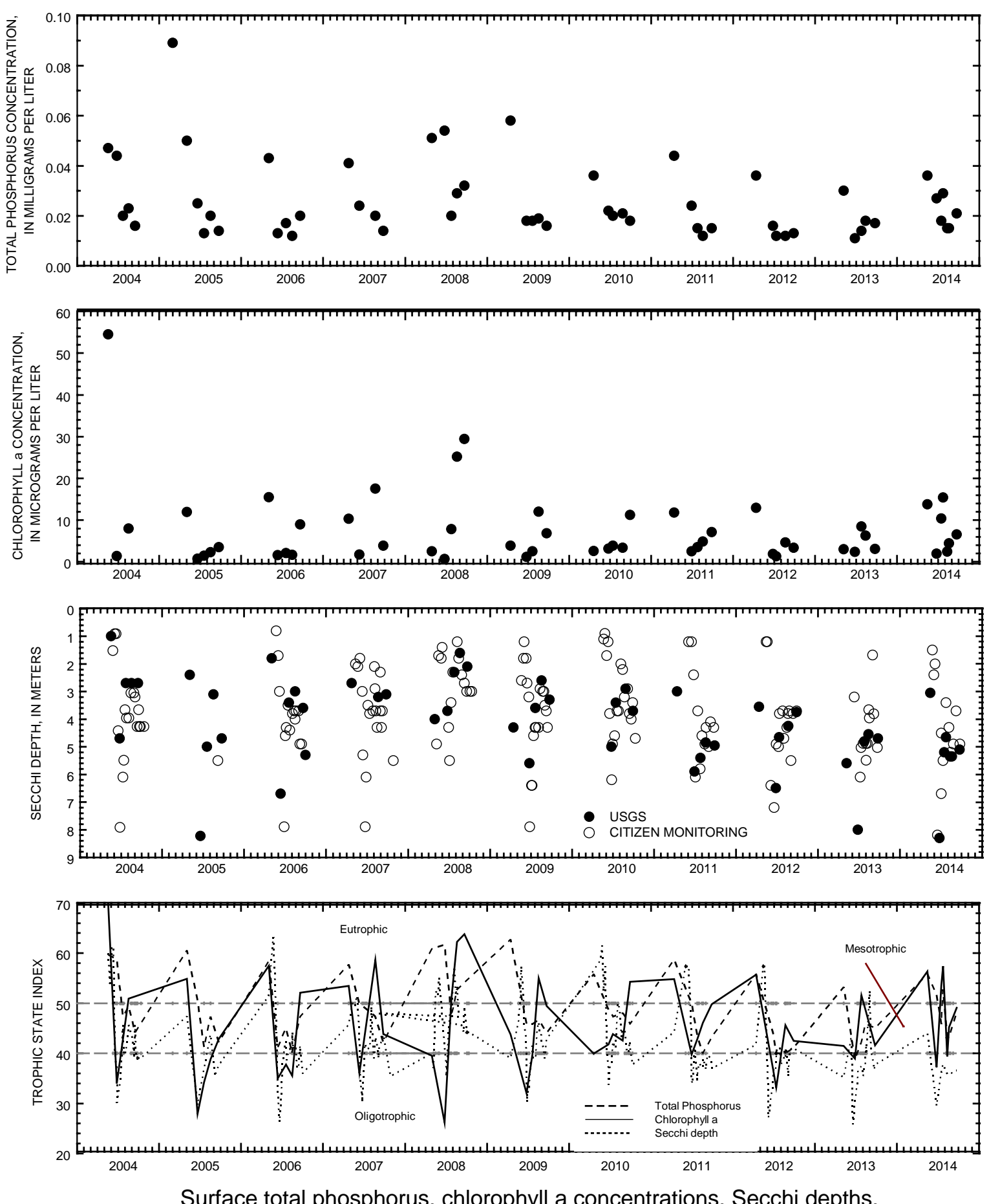

Surface total phosphorus, chlorophyll a concentrations, Secchi depths, and TSI data for Green Lake, East End, near Green Lake, Wisconsin. 
05401063 LAKE HURON NEAR PLAINFIELD, WI

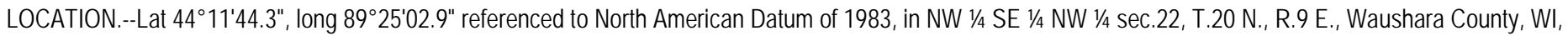
Hydrologic Unit 07070003.

DRAINAGE AREA.--8.34 mi².

PERIOD OF RECORD.--May 2010 to current year. Working records from May 1978 to April 2010 in USGS database.

GAGE.--Water-stage recorder installed November 15, 2012. Local datum used. Prior to November 15, 2012 staff gage was read periodically by observer.

COOPERATION.--Wisconsin Department of Natural Resources.

EXTREMES FOR PERIOD OF RECORD.--Maximum daily gage height, 89.01 ft., June 28, 2011; miminum daily gage height, 84.69 ft., Oct. 29-30, 2013.

29-30, 2013.

EXTREMES FOR CURRENT YEAR.--Maximum daily gage height, 85.98 ft., Jun. 19-20; miminum daily gage height, $84.69 \mathrm{ft} .$, Oct.

\begin{tabular}{|c|c|c|c|c|c|c|c|c|c|c|c|c|}
\hline \multirow[b]{2}{*}{ DAY } & \multicolumn{12}{|c|}{$\begin{array}{c}\text { GAGE HEIGHT, FEET } \\
\text { WATER YEAR OCTOBER } 2013 \text { TO SEPTEMBER } 2014 \\
\text { DAILY MEAN VALUES } \\
\text { [e, estimated] }\end{array}$} \\
\hline & OCT & NOV & DEC & JAN & FEB & MAR & APR & MAY & JUN & JUL & AUG & SEP \\
\hline 1 & 84.79 & 84.72 & 84.87 & 85.02 & 85.13 & 85.20 & 85.30 & 85.83 & 85.87 & 85.83 & 85.23 & 85.27 \\
\hline 2 & 84.78 & 84.72 & 84.88 & 85.02 & 85.13 & 85.20 & 85.30 & 85.83 & 85.94 & 85.82 & 85.26 & 85.25 \\
\hline 3 & 84.77 & 84.72 & 84.90 & 85.02 & 85.12 & 85.19 & 85.31 & 85.83 & 85.93 & 85.80 & 85.24 & 85.23 \\
\hline 4 & 84.78 & 84.71 & 84.92 & 85.02 & 85.12 & 85.20 & 85.34 & 85.82 & 85.92 & 85.77 & 85.24 & 85.21 \\
\hline 5 & 84.83 & 84.73 & 84.92 & 85.02 & 85.12 & 85.19 & 85.35 & 85.81 & 85.91 & 85.75 & 85.23 & 85.20 \\
\hline 6 & 84.92 & 84.84 & 84.91 & 85.02 & 85.12 & 85.19 & 85.35 & 85.80 & 85.90 & 85.72 & 85.20 & 85.17 \\
\hline 7 & 84.90 & 84.83 & 84.91 & 85.02 & 85.12 & 85.19 & 85.37 & 85.80 & 85.90 & 85.72 & 85.18 & 85.15 \\
\hline 8 & 84.89 & 84.83 & 84.91 & 85.02 & 85.12 & 85.19 & 85.38 & 85.80 & 85.91 & 85.76 & 85.16 & 85.13 \\
\hline 9 & 84.87 & 84.83 & 84.92 & 85.02 & 85.12 & 85.19 & 85.38 & 85.81 & 85.89 & 85.73 & 85.13 & 85.11 \\
\hline 10 & 84.86 & 84.82 & 84.92 & 85.03 & 85.12 & 85.19 & 85.39 & 85.80 & 85.87 & 85.70 & 85.11 & 85.12 \\
\hline 11 & 84.85 & 84.82 & 84.92 & 85.05 & 85.12 & 85.19 & 85.39 & 85.80 & 85.86 & 85.68 & 85.10 & 85.09 \\
\hline 12 & 84.84 & 84.81 & 84.92 & 85.05 & 85.12 & 85.19 & 85.42 & 85.88 & 85.85 & 85.66 & 85.09 & 85.06 \\
\hline 13 & 84.82 & 84.80 & 84.93 & 85.05 & 85.13 & 85.19 & 85.50 & 85.90 & 85.81 & 85.64 & 85.06 & 85.05 \\
\hline 14 & 84.80 & 84.80 & 84.93 & 85.07 & 85.13 & 85.20 & 85.63 & 85.89 & 85.79 & 85.62 & 85.03 & 85.03 \\
\hline 15 & 84.83 & 84.79 & 84.93 & 85.09 & 85.13 & 85.19 & 85.63 & 85.88 & 85.77 & 85.60 & 85.01 & 85.02 \\
\hline 16 & 84.83 & 84.82 & 84.93 & 85.09 & 85.13 & 85.18 & 85.63 & 85.86 & 85.76 & 85.57 & 84.99 & 85.01 \\
\hline 17 & 84.82 & 84.92 & 84.95 & 85.10 & 85.15 & 85.18 & 85.63 & 85.85 & 85.80 & 85.54 & 84.97 & 85.00 \\
\hline 18 & 84.81 & 84.93 & 84.95 & 85.10 & 85.17 & 85.18 & 85.62 & 85.85 & 85.96 & 85.52 & 85.00 & 84.99 \\
\hline 19 & 84.80 & 84.92 & 84.95 & 85.10 & 85.16 & 85.19 & 85.62 & 85.85 & 85.98 & 85.50 & 85.20 & 84.98 \\
\hline 20 & 84.79 & 84.91 & 84.96 & 85.10 & 85.17 & 85.19 & 85.62 & 85.87 & 85.98 & 85.48 & 85.18 & 85.04 \\
\hline 21 & 84.78 & 84.91 & 84.97 & 85.10 & 85.21 & 85.19 & 85.64 & 85.87 & 85.97 & 85.47 & 85.18 & 85.06 \\
\hline 22 & 84.77 & 84.92 & 84.98 & 85.10 & 85.20 & 85.19 & 85.63 & 85.86 & 85.96 & 85.45 & 85.18 & 85.04 \\
\hline 23 & 84.75 & 84.91 & 85.00 & 85.10 & 85.20 & 85.19 & 85.63 & 85.85 & 85.95 & 85.42 & 85.15 & 85.03 \\
\hline 24 & 84.74 & 84.89 & 85.00 & 85.10 & 85.20 & 85.18 & 85.65 & 85.84 & 85.93 & 85.40 & 85.13 & 85.02 \\
\hline 25 & 84.73 & 84.89 & 85.01 & 85.11 & 85.19 & 85.19 & 85.69 & 85.83 & 85.91 & 85.37 & 85.14 & 85.02 \\
\hline 26 & 84.72 & 84.88 & 85.02 & 85.11 & 85.19 & 85.19 & 85.68 & 85.83 & 85.90 & 85.35 & 85.13 & 85.01 \\
\hline 27 & 84.71 & 84.87 & 85.02 & 85.11 & 85.19 & 85.21 & 85.68 & 85.85 & 85.88 & 85.35 & 85.10 & 85.01 \\
\hline 28 & 84.70 & 84.88 & 85.02 & 85.11 & 85.19 & 85.28 & 85.73 & 85.84 & 85.86 & 85.32 & 85.08 & 85.00 \\
\hline 29 & 84.69 & 84.87 & 85.02 & 85.11 & --- & 85.28 & 85.79 & 85.83 & 85.86 & 85.28 & 85.09 & 85.00 \\
\hline 30 & 84.69 & 84.87 & 85.02 & 85.12 & --- & 85.28 & 85.83 & 85.83 & 85.84 & 85.26 & 85.26 & 84.98 \\
\hline 31 & 84.72 & --- & 85.02 & 85.13 & --- & 85.29 & --- & 85.81 & --- & 85.23 & 85.28 & --- \\
\hline MEAN & 84.79 & 84.84 & 84.95 & 85.07 & 85.15 & 85.20 & 85.54 & 85.84 & 85.89 & 85.56 & 85.14 & 85.08 \\
\hline MAX & 84.92 & 84.93 & 85.02 & 85.13 & 85.21 & 85.29 & 85.83 & 85.90 & 85.98 & 85.83 & 85.28 & 85.27 \\
\hline MIN & 84.69 & 84.71 & 84.87 & 85.02 & 85.12 & 85.18 & 85.30 & 85.80 & 85.76 & 85.23 & 84.97 & 84.98 \\
\hline
\end{tabular}




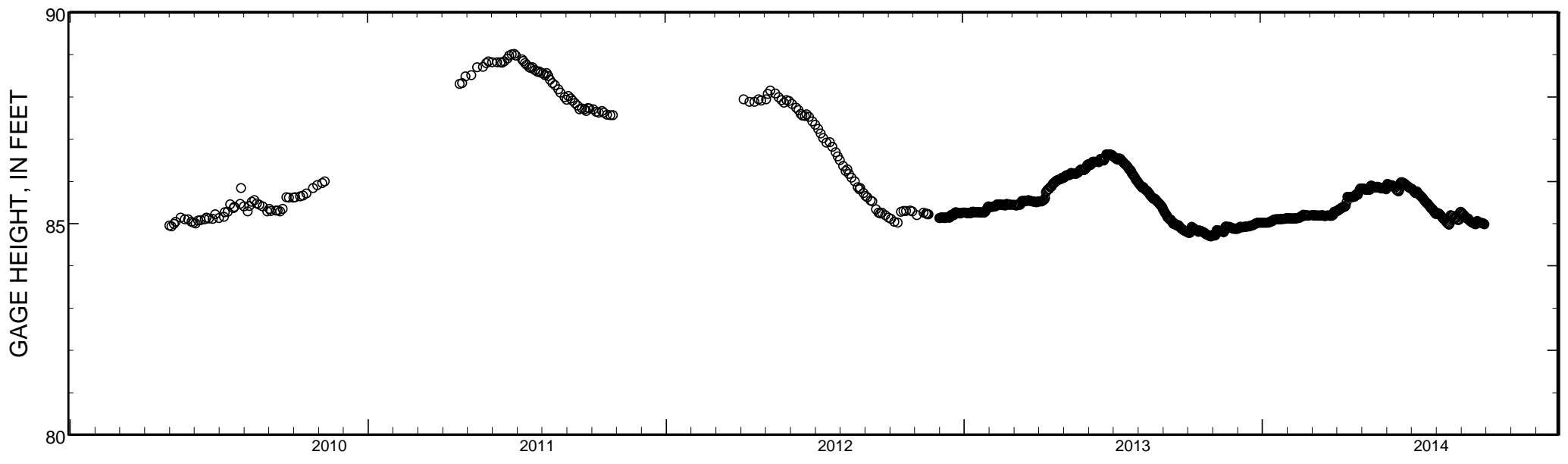

Stage hydrograph for Lake Huron near Plainfield, 2010-2014.

86 


\section{LAKE KEGONSA AT BARBER DRIVE NEAR STOUGHTON, WI}

LOCATION.--Lat 42 $57^{\prime} 15^{\prime \prime}$, long 89 $16^{\prime} 47^{\prime \prime}$ referenced to North American Datum of 1927, in SW 1/4 NE 1/4 NE 1/4 sec.26, T.6 N., R.10 E., Dane County, WI, Hydrologic Unit 07090001, on downstream side of bridge on Barber Drive, 3.5 mi northwest of Stoughton.

SURFACE AREA.--1.05 $\mathrm{mi}^{2}$.

DRAINAGE AREA.--386 mi².

PERIOD OF RECORD.--October 2003 to current year.

GAGE.--Water-stage recorder. Datum of gage is $840.00 \mathrm{ft}$ above sea level (levels from Wisconsin Department of Transportation benchmark).

EXTREMES FOR PERIOD OF RECORD.--Maximum gage height observed, $5.73 \mathrm{ft}$, June 16, 2008; minimum observed, $2.07 \mathrm{ft}$, Jan.27, 2006.

EXTREMES FOR CURRENT YEAR.--Maximum gage height observed, $3.85 \mathrm{ft}$, July 2 and 3; minimum observed, $2.37 \mathrm{ft}$, Jan. 29.

GAGE HEIGHT, FEET

WATER YEAR OCTOBER 2013 TO SEPTEMBER 2014

DAILY MEAN VALUES

\begin{tabular}{|c|c|c|c|c|c|c|c|c|c|c|c|c|}
\hline DAY & OCT & NOV & DEC & JAN & FEB & MAR & APR & MAY & JUN & JUL & AUG & SEP \\
\hline 1 & 3.41 & 2.97 & 2.89 & 2.65 & --- & --. & 2.55 & 3.43 & 3.33 & 3.82 & 3.46 & e3.24 \\
\hline 2 & 3.42 & 2.94 & 2.90 & 2.63 & --- & --- & 2.55 & 3.41 & 3.41 & 3.85 & 3.48 & --- \\
\hline 3 & 3.42 & 2.90 & 2.91 & 2.62 & --- & --- & 2.54 & 3.39 & 3.46 & 3.85 & 3.50 & --- \\
\hline 4 & 3.45 & 2.86 & 2.92 & 2.59 & --- & --- & 2.54 & 3.36 & 3.51 & 3.84 & 3.54 & --- \\
\hline 5 & 3.48 & 2.83 & 2.96 & 2.55 & -- & -- & 2.54 & 3.33 & 3.55 & 3.81 & 3.59 & 3.25 \\
\hline 6 & 3.50 & 2.85 & 3.02 & 2.54 & --- & --- & 2.54 & 3.28 & 3.56 & 3.78 & 3.59 & 3.22 \\
\hline 7 & 3.50 & 2.84 & 3.06 & 2.51 & --- & --- & 2.54 & 3.24 & 3.57 & 3.75 & 3.57 & 3.20 \\
\hline 8 & 3.51 & 2.83 & 3.07 & 2.50 & --- & 2.43 & 2.56 & 3.20 & 3.59 & 3.76 & 3.54 & 3.17 \\
\hline 9 & 3.52 & 2.80 & 3.09 & 2.49 & --- & 2.42 & 2.64 & 3.21 & 3.57 & 3.71 & 3.51 & 3.14 \\
\hline 10 & 3.52 & 2.80 & 3.09 & 2.48 & --- & 2.41 & 2.71 & 3.23 & 3.55 & 3.65 & 3.48 & 3.17 \\
\hline 11 & 3.53 & 2.80 & 3.09 & 2.47 & --- & 2.42 & 2.76 & 3.25 & 3.54 & 3.59 & 3.47 & 3.17 \\
\hline 12 & 3.51 & 2.78 & 3.08 & 2.46 & --- & 2.43 & 2.82 & 3.32 & 3.50 & 3.52 & 3.44 & 3.15 \\
\hline 13 & 3.50 & 2.75 & 3.06 & 2.45 & --. & 2.45 & 2.95 & 3.44 & 3.47 & 3.48 & 3.41 & 3.13 \\
\hline 14 & 3.50 & 2.74 & 3.03 & 2.44 & --- & 2.49 & 3.25 & 3.48 & 3.43 & 3.41 & 3.39 & 3.11 \\
\hline 15 & 3.50 & 2.73 & 3.01 & 2.44 & --- & 2.53 & 3.31 & 3.51 & 3.39 & 3.35 & 3.37 & 3.10 \\
\hline 16 & 3.50 & 2.72 & 2.99 & 2.44 & --- & 2.59 & 3.34 & 3.50 & 3.36 & 3.30 & 3.37 & 3.12 \\
\hline 17 & 3.49 & 2.80 & 2.98 & 2.43 & --- & 2.64 & 3.35 & 3.49 & 3.48 & 3.25 & 3.38 & 3.13 \\
\hline 18 & 3.46 & 2.86 & 2.95 & 2.43 & --- & 2.66 & 3.36 & 3.47 & 3.53 & 3.23 & 3.37 & 3.15 \\
\hline 19 & 3.39 & 2.89 & 2.93 & 2.43 & 2.37 & 2.67 & 3.34 & 3.45 & 3.54 & 3.20 & 3.41 & 3.14 \\
\hline 20 & 3.32 & 2.90 & 2.91 & 2.41 & 2.37 & 2.69 & 3.31 & 3.43 & 3.70 & 3.17 & 3.42 & 3.15 \\
\hline 21 & 3.26 & 2.91 & 2.89 & 2.42 & 2.39 & 2.70 & 3.31 & 3.41 & 3.75 & 3.14 & 3.47 & 3.17 \\
\hline 22 & 3.21 & 2.91 & 2.89 & 2.42 & 2.40 & 2.70 & 3.30 & 3.39 & 3.77 & 3.13 & 3.52 & 3.17 \\
\hline 23 & 3.16 & 2.90 & 2.87 & 2.41 & 2.42 & 2.69 & 3.28 & 3.36 & 3.78 & 3.17 & 3.54 & 3.18 \\
\hline 24 & 3.11 & 2.86 & 2.84 & 2.41 & 2.43 & 2.67 & 3.29 & 3.31 & 3.77 & 3.11 & 3.55 & 3.18 \\
\hline 25 & 3.07 & 2.90 & 2.82 & 2.39 & 2.43 & 2.64 & 3.32 & 3.26 & 3.78 & 3.09 & 3.65 & 3.18 \\
\hline 26 & 3.04 & 2.94 & 2.78 & 2.39 & --- & 2.60 & 3.33 & 3.23 & 3.76 & 3.17 & 3.65 & 3.18 \\
\hline 27 & 3.02 & 2.89 & 2.75 & 2.39 & --- & 2.58 & 3.34 & 3.23 & 3.74 & 3.24 & 3.57 & 3.18 \\
\hline 28 & 3.02 & 2.86 & 2.73 & 2.38 & --. & 2.59 & 3.39 & 3.27 & 3.70 & 3.29 & 3.46 & 3.17 \\
\hline 29 & 2.99 & 2.87 & 2.70 & 2.37 & --- & 2.58 & 3.41 & 3.29 & 3.68 & 3.34 & 3.37 & 3.17 \\
\hline 30 & 2.98 & 2.88 & 2.68 & 2.38 & --- & 2.56 & 3.44 & 3.32 & 3.76 & 3.39 & 3.33 & 3.16 \\
\hline 31 & 2.99 & -- & 2.67 & 2.37 & --- & 2.56 & --- & 3.33 & --- & 3.43 & 3.28 & --- \\
\hline MEAN & 3.33 & 2.85 & 2.92 & 2.46 & --- & --. & 3.03 & 3.35 & 3.58 & 3.45 & 3.47 & --- \\
\hline MAX & 3.53 & 2.97 & 3.09 & 2.65 & --- & --- & 3.44 & 3.51 & 3.78 & 3.85 & 3.65 & --- \\
\hline MIN & 2.98 & 2.72 & 2.67 & 2.37 & -.- & --.- & 2.54 & 3.20 & 3.33 & 3.09 & 3.28 & -.-- \\
\hline
\end{tabular}




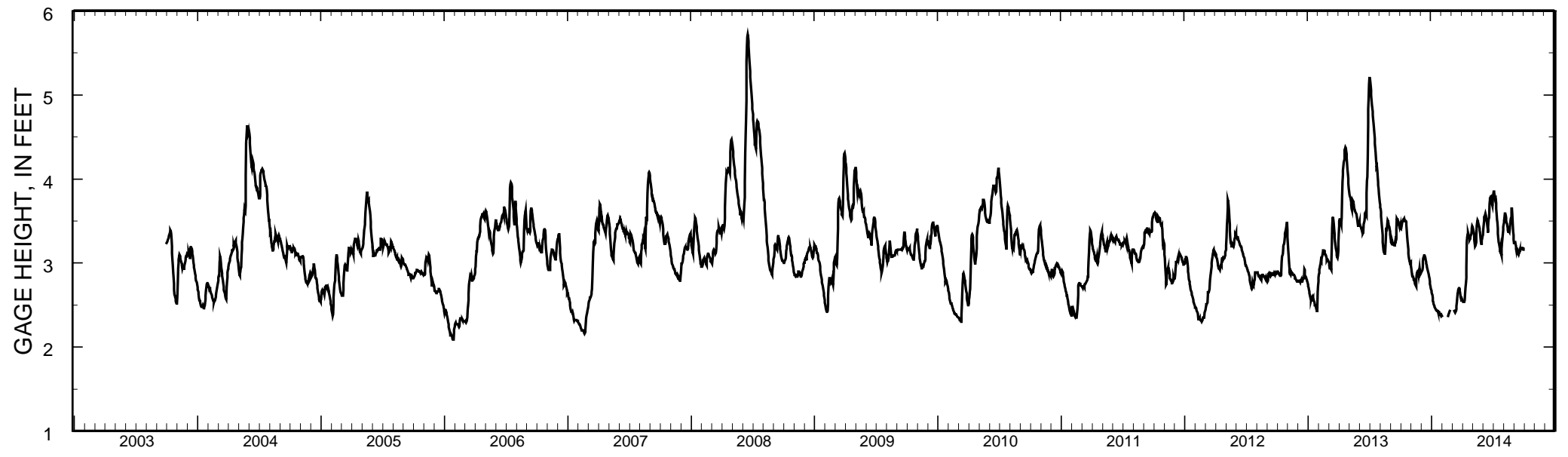

Stage hydrograph for Lake Kegonsa, 1993-2014. 


\section{LAKE KOSHKONONG NEAR NEWVILLE, WI}

LOCATION.--Lat 42 $51^{\prime} 27^{\prime \prime}$, long $88^{\circ} 56^{\prime} 27^{\prime \prime}$ referenced to North American Datum of 1927, in NW 1/4 NE 1/4 sec.34, T.5 N., R.13 E., Jefferson County, WI, Hydrologic Unit 07090001, $80 \mathrm{ft}$ east of Pottawatomi Trail Bridge at Bingham Point Estates, and 4.5 mi northeast of Newville.

SURFACE AREA.-16.34 mi2.

DRAINAGE AREA.--2,560 $\mathrm{mi}^{2}$

PERIOD OF RECORD.--July 1987 to current year.

GAGE.--Water-stage recorder. Datum of gage is $769.77 \mathrm{ft}$ above NAVD of 1988 (Wisconsin Department of Transportation bench mark).

REMARKS.--Lake level regulated by dam at Indianford. Gage-height telemeter at station.

EXTREMES FOR PERIOD OF RECORD.--Maximum recorded gage height, 15.13ft, June 21, 22, 2008; minimum recorded, $4.98 \mathrm{ft}$, Dec. 6 and $9,2012$.

EXTREMES FOR CURRENT YEAR.--Maximum recorded gage height, $9.71 \mathrm{ft}$, May 17; minimum recorded gage height, 5.62 ft, Feb. 16.

GAGE HEIGHT, FEET

WATER YEAR OCTOBER 2013 TO SEPTEMBER 2014

DAILY MEAN VALUES

\begin{tabular}{|c|c|c|c|c|c|c|c|c|c|c|c|c|}
\hline \multicolumn{13}{|c|}{ [e, estimated] } \\
\hline DAY & OCT & NOV & DEC & JAN & FEB & MAR & APR & MAY & JUN & JUL & AUG & SEP \\
\hline 2 & 6.23 & 5.99 & 6.62 & 6.12 & 5.86 & 5.85 & 8.36 & 9.41 & 8.11 & 9.35 & 7.18 & 6.30 \\
\hline 4 & 6.17 & 6.04 & 6.64 & 6.08 & 5.81 & 5.83 & 8.37 & 9.37 & 7.99 & 9.49 & 6.99 & 6.34 \\
\hline 5 & 6.18 & 6.10 & 6.67 & 6.02 & 5.80 & 5.83 & 8.35 & 9.31 & 7.95 & 9.51 & 6.95 & 6.43 \\
\hline 6 & 6.23 & 6.22 & 6.65 & 5.98 & 5.79 & 5.82 & 8.31 & 9.24 & 7.89 & 9.53 & 6.85 & 6.38 \\
\hline 8 & 6.29 & 6.28 & 6.60 & 5.94 & 5.76 & 5.78 & 8.21 & 9.09 & 7.78 & 9.63 & 6.60 & 6.22 \\
\hline 9 & 6.33 & 6.36 & 6.59 & 5.92 & 5.75 & 5.78 & 8.15 & 9.02 & 7.71 & 9.60 & 6.48 & 6.16 \\
\hline 10 & 6.38 & 6.37 & 6.57 & 5.91 & 5.73 & 5.79 & 8.10 & 8.95 & 7.65 & 9.54 & 6.36 & 6.22 \\
\hline 11 & 6.41 & 6.41 & 6.56 & 5.92 & 5.72 & 5.80 & 8.05 & 8.88 & 7.63 & 9.47 & 6.28 & 6.21 \\
\hline 12 & 6.45 & 6.41 & 6.53 & 5.91 & 5.70 & 5.86 & 8.00 & 8.94 & 7.58 & 9.43 & 6.25 & 6.20 \\
\hline 13 & 6.48 & 6.39 & 6.50 & 5.91 & 5.68 & 5.93 & 8.03 & 9.21 & 7.53 & 9.55 & 6.17 & 6.21 \\
\hline 17 & 6.30 & 6.47 & 6.41 & 5.94 & 5.63 & 6.64 & 8.85 & 9.71 & 7.37 & 9.17 & 6.20 & 6.24 \\
\hline 18 & 6.25 & 6.55 & 6.38 & 5.95 & 5.63 & 6.85 & 9.05 & 9.69 & 7.45 & 9.04 & 6.24 & 6.24 \\
\hline 19 & 6.20 & 6.53 & 6.36 & 5.96 & 5.64 & 7.05 & 9.18 & 9.63 & 7.64 & 8.91 & 6.32 & 6.22 \\
\hline 20 & 6.15 & 6.56 & 6.33 & 5.97 & 5.67 & 7.22 & 9.28 & 9.57 & 7.97 & 8.78 & 6.28 & 6.24 \\
\hline 21 & 6.15 & 6.63 & 6.31 & 5.97 & 5.70 & 7.38 & 9.35 & 9.48 & 8.23 & 8.65 & 6.26 & 6.28 \\
\hline 22 & 6.09 & 6.72 & 6.31 & 5.98 & 5.72 & 7.53 & 9.41 & 9.35 & 8.44 & 8.53 & 6.26 & 6.24 \\
\hline 23 & 6.07 & 6.79 & 6.29 & 5.98 & 5.74 & 7.65 & 9.42 & 9.21 & 8.62 & 8.40 & 6.25 & 6.22 \\
\hline 24 & 6.04 & 6.75 & 6.26 & 5.98 & 5.77 & 7.74 & 9.44 & 9.07 & 8.78 & 8.25 & 6.26 & 6.20 \\
\hline 25 & 5.99 & 6.72 & 6.24 & 5.97 & 5.80 & 7.81 & 9.47 & 8.93 & 8.87 & 8.11 & 6.36 & 6.19 \\
\hline 26 & 5.99 & 6.70 & 6.21 & 5.96 & 5.82 & 7.86 & 9.46 & 8.78 & 8.94 & 8.00 & 6.45 & 6.18 \\
\hline 27 & 5.95 & 6.68 & 6.19 & 5.95 & 5.84 & 7.90 & 9.41 & 8.66 & 8.98 & 7.91 & 6.42 & 6.17 \\
\hline
\end{tabular}




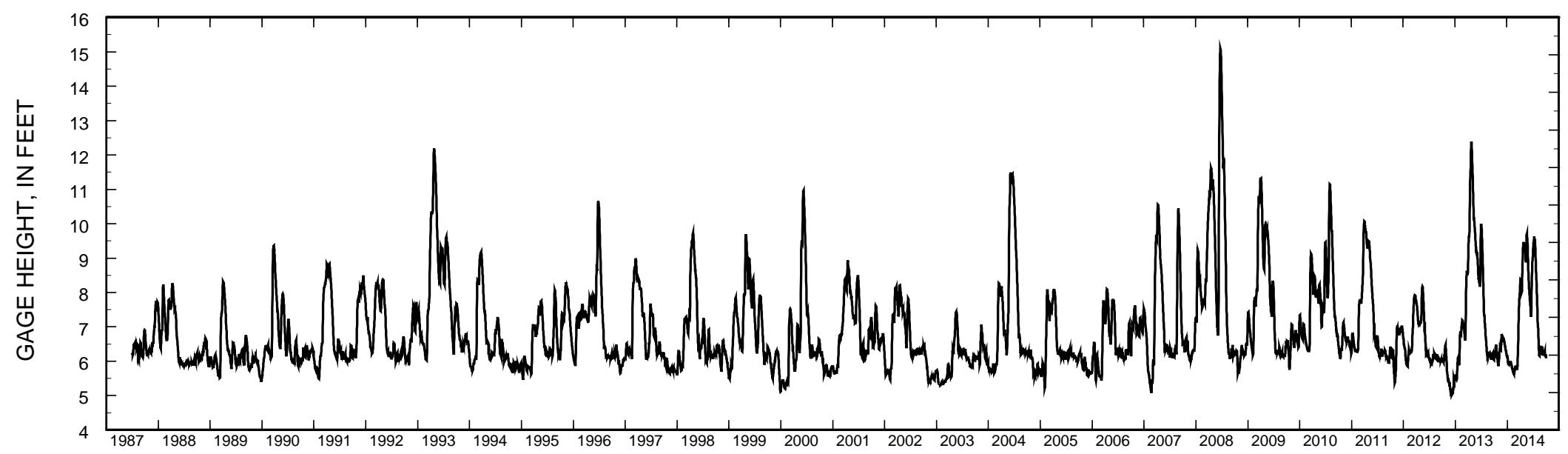

Stage hydrograph for Lake Koshkonong, 1987-2014. 
LOCATION.--Lat 44¹2'56.6", long 8907'14.6" referenced to North American Datum of 1983, in SE 1/4 SW 1/4 Sec.7, T.20 N., R.12 E., Waushara County, WI, Hydrologic Unit 04030202.

PERIOD OF RECORD.--April 2010 to current year.

GAGE.--A staff gage is periodically read by an observer, and reported in local datum.

COOPERATION.--Wisconsin Department of Natural Resources.

REMARKS.--Lake has no outlet.

EXTREMES FOR PERIOD OF RECORD.--Maximum observed gage height, $84.66 \mathrm{ft}$ May 6, 2012; miminum observed gage height, $82.72 \mathrm{ft}$ Apr. $23,2010$. Jul. 26 and Sept. 27.

EXTREMES FOR CURRENT YEAR.--Maximum observed gage height, $84.47 \mathrm{ft}$ May 24; miminum observed gage height, $84.06 \mathrm{ft}$

GAGE HEIGHT, FEET

WATER YEAR OCTOBER 2013 TO SEPTEMBER 2014

\begin{tabular}{|c|c|c|c|c|c|c|c|c|c|c|c|c|}
\hline \multicolumn{13}{|c|}{ [e, estimated] } \\
\hline DAY & OCT & NOV & DEC & JAN & FEB & MAR & APR & MAY & JUN & JUL & AUG & SEP \\
\hline 1 & --- & -- & --- & --- & -- & --- & --- & -- & --- & -- & --- & -- \\
\hline 2 & --- & --- & --- & --- & --- & --- & --- & -- & -- & -- & -- & -- \\
\hline 3 & --- & --- & --- & --- & -- & --- & --- & --- & -- & -- & -- & -- \\
\hline 4 & --- & --- & --- & --- & -- & --- & --- & -- & -- & 84.29 & --- & -- \\
\hline 5 & --- & -- & --- & --- & --- & --- & --- & --- & --- & -- & --- & --- \\
\hline 6 & 84.15 & -- & --- & --- & --- & --- & --- & --- & 84.25 & --- & --- & 84.18 \\
\hline 7 & --- & --- & --- & --- & --- & --- & --- & --- & --- & --- & --- & --- \\
\hline 8 & --- & --- & --- & --- & --- & --- & --- & --- & --- & --- & 83.96 & -- \\
\hline 9 & --- & --- & --- & --- & --- & --- & --- & --- & --- & --- & --- & --- \\
\hline 10 & --- & --- & --- & --- & --- & --- & --- & --- & --- & --- & --- & --- \\
\hline 11 & --- & -- & --- & --- & --- & -- & --- & -- & -- & 84.23 & -- & -- \\
\hline 12 & --- & --- & --- & --- & -- & --- & --- & --- & --- & --- & --- & --- \\
\hline 13 & 84.10 & --- & --- & --- & -- & --- & --- & 84.45 & 84.21 & -- & --- & --- \\
\hline 14 & --- & --- & --- & --- & --- & --- & --- & -- & -- & -- & --- & -- \\
\hline 15 & --- & --- & --- & --- & --- & --- & --- & --- & --- & --- & 83.90 & --- \\
\hline 16 & --- & --- & --- & --- & --- & --- & --- & --- & -- & -- & --- & -- \\
\hline 17 & -- & -- & --- & --- & --- & --- & -- & 84.45 & -- & -- & -- & -- \\
\hline 18 & --- & -- & --- & --- & -- & --- & --- & --- & --- & 84.12 & -- & -- \\
\hline 19 & --- & --- & --- & --- & --- & --- & --- & --- & --- & --- & --- & --- \\
\hline 20 & 84.13 & --- & --- & --- & --- & --- & --- & --- & 84.39 & -- & --- & -- \\
\hline 21 & --- & -- & --- & --- & --- & --- & --- & -- & -- & -- & --- & 84.20 \\
\hline 22 & 84.15 & --- & --- & --- & -- & --- & --- & -- & --- & -- & 84.14 & -- \\
\hline 23 & -- & -- & --- & --- & -- & --- & -- & --- & -- & -- & -- & -- \\
\hline 24 & --- & --- & --- & --- & --- & --- & --- & 84.47 & --- & --- & --- & --- \\
\hline 25 & --- & --- & --- & --- & --- & --- & --- & --- & --- & --- & --- & --- \\
\hline 26 & -- & -- & --- & --- & --- & --- & --- & -- & -- & 84.06 & --- & -- \\
\hline 27 & --- & --- & --- & --- & --- & --- & --- & --- & --- & --- & --- & 84.06 \\
\hline 28 & --- & -- & --- & --- & --- & --- & --- & -- & 84.33 & --- & --- & -- \\
\hline 29 & --- & --- & --- & --- & --- & --- & --- & --- & --- & --- & 84.16 & --- \\
\hline 30 & --- & --- & --- & --- & --- & --- & --- & -- & --- & --- & --- & --- \\
\hline 31 & --- & --- & --- & --- & --- & --- & --- & 84.33 & --- & --- & --- & --- \\
\hline
\end{tabular}




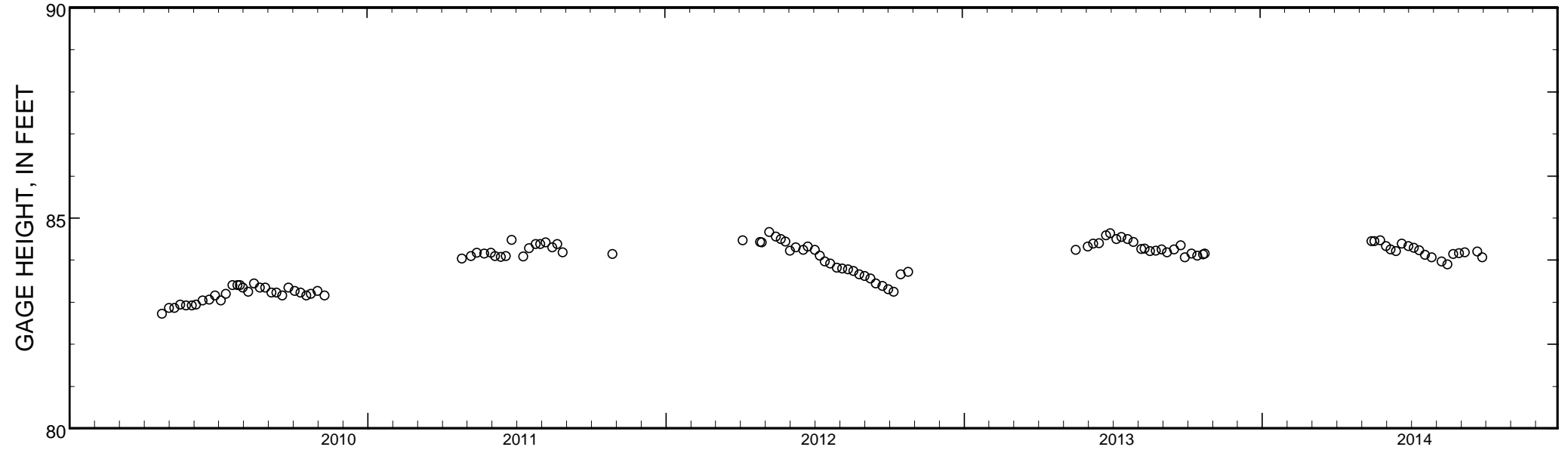

Stage hydrograph for Long Lake, 2010-2014. 


\section{LAKE MENDOTA AT MADISON, WI}

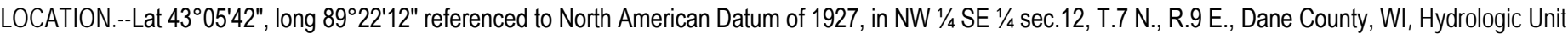
07090001, in county boat house at dam at outlet, in Madison.

SURFACE AREA.-15.2 mi².

DRAINAGE AREA.--233 mi² of which $36.6 \mathrm{mi}^{2}$ probably is noncontributing.

PERIOD OF RECORD.--January 1916 to January 1985 (incomplete), February 1985 to current year.

REVISED RECORDS.--WDR WI-73-1: Drainage area.

GAGE.--Water-stage recorder. Datum of gage is $840.00 \mathrm{ft}$ above NGVD of 1929, or $5.60 \mathrm{ft}$ below City of Madison datum. Prior to Oct. 1, 1979, gage datum was $847.82 \mathrm{ft}$; prior to Nov. 15, 1971, nonrecording gage at same site at the higher datum.

REMARKS.--Lake level regulated by concrete dam with two 12-foot gates and 20-foot lock at outlet. Gage-height telemeter at station.

EXTREMES FOR PERIOD OF RECORD.--Maximum gage height observed, $12.75 \mathrm{ft}$, June 5, 2000; minimum observed, $8.02 \mathrm{ft}$, Feb. 24 to Mar. 10, 1920, current datum.

EXTREMES FOR CURRENT YEAR.--Maximum recorded gage height, $10.89 \mathrm{ft}$, July 2; minimum recorded, $8.34 \mathrm{ft}$, Mar. 9 and 10.

GAGE HEIGHT, FEET

WATER YEAR OCTOBER 2013 TO SEPTEMBER 2014

DAILY MEAN VALUES

\begin{tabular}{|c|c|c|c|c|c|c|c|c|c|c|c|c|}
\hline DAY & ОСт & NOV & DEC & JAN & FEB & MAR & APR & MAY & JUN & JUL & AUG & SEP \\
\hline 1 & 10.08 & 9.64 & 8.71 & 8.57 & 8.48 & 8.37 & 9.19 & 9.96 & 9.81 & 10.87 & 9.99 & 10.03 \\
\hline 2 & 10.08 & 9.61 & 8.67 & 8.57 & 8.48 & 8.37 & 9.21 & 9.96 & 10.04 & 10.89 & 9.99 & 10.03 \\
\hline 3 & 10.08 & 9.57 & 8.65 & 8.56 & 8.48 & 8.36 & 9.22 & 9.95 & 10.13 & 10.87 & 9.97 & 10.01 \\
\hline 4 & 10.11 & 9.52 & 8.64 & 8.56 & 8.47 & 8.36 & 9.25 & 9.92 & 10.14 & 10.84 & 9.99 & 10.11 \\
\hline 5 & 10.14 & 9.51 & 8.66 & 8.55 & 8.47 & 8.37 & 9.26 & 9.90 & 10.15 & 10.80 & 10.08 & 10.21 \\
\hline 6 & 10.14 & 9.56 & 8.62 & 8.54 & 8.46 & 8.37 & 9.28 & 9.86 & 10.13 & 10.77 & 10.10 & 10.20 \\
\hline 7 & 10.13 & 9.54 & 8.54 & 8.54 & 8.46 & 8.36 & 9.30 & 9.84 & 10.12 & 10.76 & 10.08 & 10.19 \\
\hline 8 & 10.11 & 9.49 & 8.58 & 8.54 & 8.46 & 8.35 & 9.32 & 9.81 & 10.10 & 10.77 & 10.06 & 10.17 \\
\hline 9 & 10.09 & 9.48 & 8.63 & 8.54 & 8.46 & 8.34 & 9.33 & 9.80 & 10.07 & 10.72 & 10.03 & 10.15 \\
\hline 10 & 10.08 & 9.44 & 8.63 & 8.54 & 8.46 & 8.34 & 9.35 & 9.78 & 10.05 & 10.68 & 10.01 & 10.20 \\
\hline 11 & 10.07 & 9.41 & 8.63 & 8.53 & 8.46 & 8.35 & 9.36 & 9.76 & 10.02 & 10.63 & 10.00 & 10.17 \\
\hline 12 & 10.06 & 9.37 & 8.63 & 8.50 & 8.46 & 8.39 & 9.38 & 9.81 & 10.02 & 10.61 & 9.98 & 10.15 \\
\hline 13 & 10.04 & 9.31 & 8.63 & 8.50 & 8.42 & 8.44 & 9.52 & 9.90 & 9.98 & 10.61 & 9.94 & 10.13 \\
\hline 14 & 10.01 & 9.27 & 8.62 & 8.50 & 8.40 & 8.49 & 9.83 & 9.91 & 9.94 & 10.58 & 9.91 & 10.10 \\
\hline 15 & 9.99 & 9.23 & 8.62 & 8.50 & 8.40 & 8.60 & 9.93 & 9.89 & 9.90 & 10.54 & 9.88 & 10.10 \\
\hline 16 & 9.99 & 9.19 & 8.62 & 8.50 & 8.39 & 8.72 & 9.94 & 9.87 & 9.89 & 10.49 & 9.86 & 10.10 \\
\hline 17 & 9.97 & 9.25 & 8.61 & 8.50 & 8.37 & 8.81 & 9.95 & 9.84 & 9.99 & 10.45 & 9.83 & 10.09 \\
\hline 18 & 9.95 & 9.28 & 8.59 & 8.50 & 8.37 & 8.86 & 9.94 & 9.81 & 10.04 & 10.41 & 9.81 & 10.08 \\
\hline 19 & 9.92 & 9.21 & 8.55 & 8.49 & 8.37 & 8.91 & 9.92 & 9.78 & 10.16 & 10.36 & 9.87 & 10.06 \\
\hline 20 & 9.90 & 9.17 & 8.55 & 8.49 & 8.39 & 8.96 & 9.91 & 9.79 & 10.37 & 10.31 & 9.86 & 10.07 \\
\hline 21 & 9.89 & 9.15 & 8.54 & 8.49 & 8.41 & 9.01 & 9.90 & 9.80 & 10.47 & 10.27 & 9.88 & 10.08 \\
\hline 22 & 9.85 & 9.13 & 8.57 & 8.49 & 8.41 & 9.05 & 9.88 & 9.78 & 10.53 & 10.26 & 9.91 & 10.05 \\
\hline 23 & 9.82 & 9.09 & 8.57 & 8.49 & 8.41 & 9.06 & 9.85 & 9.76 & 10.56 & 10.24 & 9.90 & 10.04 \\
\hline 24 & 9.80 & 9.01 & 8.57 & 8.49 & 8.40 & 9.06 & 9.85 & 9.74 & 10.58 & 10.20 & 9.89 & 10.03 \\
\hline 25 & 9.76 & 8.97 & 8.57 & 8.48 & 8.39 & 9.07 & 9.87 & 9.72 & 10.56 & 10.16 & 9.97 & 10.02 \\
\hline 26 & 9.74 & 8.94 & 8.57 & 8.48 & 8.39 & 9.07 & 9.85 & 9.72 & 10.54 & 10.14 & 10.04 & 10.01 \\
\hline 27 & 9.70 & 8.89 & 8.56 & 8.48 & 8.38 & 9.07 & 9.82 & 9.77 & 10.52 & 10.14 & 10.03 & 10.01 \\
\hline 28 & 9.67 & 8.84 & 8.56 & 8.48 & 8.38 & 9.13 & 9.85 & 9.86 & 10.51 & 10.10 & 10.01 & 10.00 \\
\hline 29 & 9.64 & 8.79 & 8.56 & 8.48 & --- & 9.15 & 9.94 & 9.85 & 10.54 & 10.06 & 9.99 & 10.00 \\
\hline 30 & 9.62 & 8.75 & 8.56 & 8.48 & --- & 9.16 & 9.96 & 9.83 & 10.70 & 10.03 & 10.01 & 9.97 \\
\hline 31 & 9.63 & -- & 8.56 & 8.48 & --- & 9.17 & --- & 9.81 & --- & 10.01 & 10.02 & --- \\
\hline MEAN & 9.94 & 9.25 & 8.60 & 8.51 & 8.42 & 8.71 & 9.64 & 9.83 & 10.22 & 10.47 & 9.96 & 10.09 \\
\hline MAX & 10.14 & 9.64 & 8.71 & 8.57 & 8.48 & 9.17 & 9.96 & 9.96 & 10.70 & 10.89 & 10.10 & 10.21 \\
\hline MIN & 9.62 & 8.75 & 8.54 & 8.48 & 8.37 & 8.34 & 9.19 & 9.72 & 9.81 & 10.01 & 9.81 & 9.97 \\
\hline
\end{tabular}




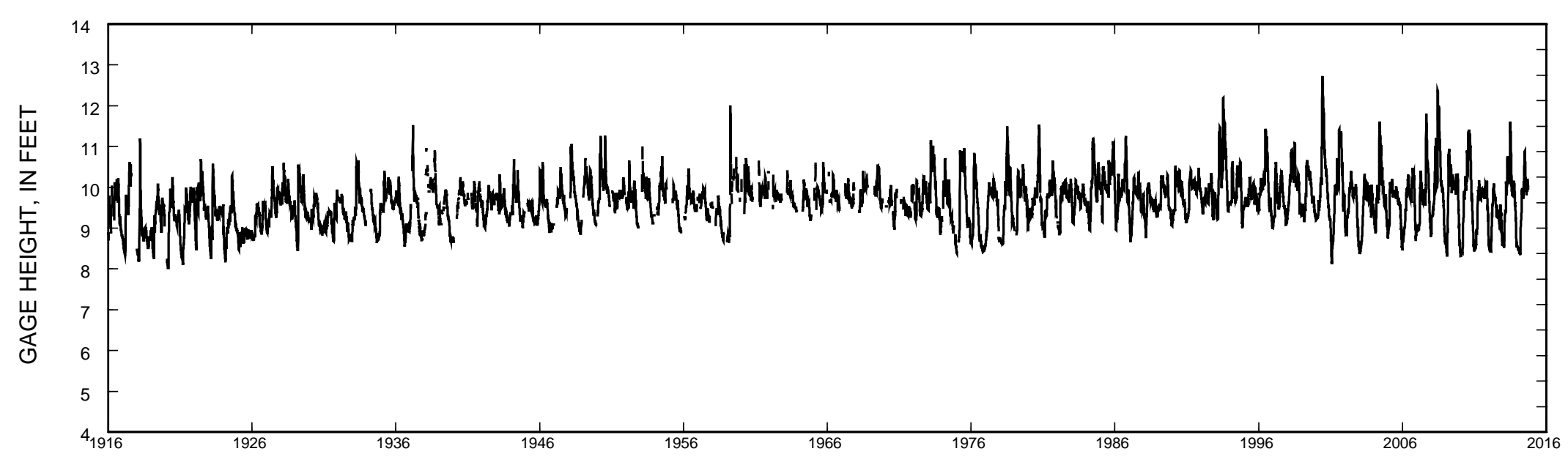

tage hydrograph for Lake Mendota, 1916-2014

94 
430251088284700 MIDDLE GENESEE LAKE, AT GENESEE LAKE ROAD, NEAR OCONOMOWOC, WI

LOCATION.--Lat $43^{\circ} 02^{\prime} 51^{\prime \prime}$, long $88^{\circ} 28^{\prime} 47^{\prime \prime}$, in SW $1 / 4$ SW 1/4 SW 1/4 Sec.22, T. 7 N., R.17 E., Waukesha County, Hydrologic Unit 07090001, at the southwest side of the lake about 2 miles south of Oconomowoc.

SURFACE AREA.--0.17 $\mathrm{mi}^{2}$.

PERIOD OF RECORD.--April 1996 to current year.

GAGE.--Staff gage. Local observer, Tom Schubring provided most readings of gage. Datum of gage is about $0.0 \mathrm{ft}$ above NGVD of 1929 .

EXTREMES FOR THE PERIOD OF RECORD.--Maximum observed gage height, $869.65 \mathrm{ft}$, July 12, 2008; minimum observed, $863.88 \mathrm{ft}, 0 \mathrm{ct}$. $31,2005$.

EXTREMES FOR CURRENT YEAR.--Maximum observed gage height, 866.86 ft, May 26; minimum observed, 865.82 ft, Sept. 28.

GAGE HEIGHT, FEET

WATER YEAR OCTOBER 2013 TO SEPTEMBER 2014

\begin{tabular}{|c|c|c|c|c|c|c|c|c|c|c|c|c|}
\hline DAY & OCT & NOV & DEC & JAN & FEB & MAR & APR & MAY & JUN & JUL & AUG & SEP \\
\hline 1 & 866.61 & --- & --- & --- & --- & --- & --- & --- & 866.80 & --- & --- & --- \\
\hline 2 & --- & 866.51 & --- & --- & --- & --- & --- & --- & 866.84 & --- & --- & --- \\
\hline 3 & --- & --- & --- & --- & --- & --- & --- & --- & --- & --- & 866.42 & 866.24 \\
\hline 4 & -- & 866.49 & --- & --- & -- & --- & -- & -- & --- & 866.82 & -- & -- \\
\hline 5 & -- & --- & --- & --- & -- & --- & --- & --- & --- & --- & --- & --- \\
\hline 6 & 866.67 & -- & --- & --- & -- & --- & -- & -- & --- & 866.76 & -- & -- \\
\hline 7 & $--\cdot$ & 866.53 & -- & --- & --- & -- & -- & -- & 866.74 & -- & -- & -- \\
\hline 8 & --- & --- & --- & --- & --- & --- & --- & --- & --- & --- & --- & 866.10 \\
\hline 9 & --- & --- & --- & --- & --- & --- & --- & --- & --- & --- & --- & --- \\
\hline 10 & -- & -- & --- & --- & -- & --- & -- & -- & --- & -- & -- & -- \\
\hline 11 & --- & --- & --- & --- & --- & --- & --- & --- & --- & --- & 866.32 & -- \\
\hline 12 & 866.57 & --- & --- & --- & --- & --- & --- & --- & 866.72 & 866.72 & -- & --- \\
\hline 13 & --- & 866.47 & --- & --- & --- & --- & --- & --- & --- & --- & --- & 866.04 \\
\hline 14 & -- & -- & --- & -- & -- & --- & -- & -- & 866.68 & --- & -- & -- \\
\hline 15 & -- & -- & --- & --- & -- & -- & -- & 866.84 & --- & -- & -- & -- \\
\hline 16 & --- & --- & --- & --- & --- & --- & --- & --- & 866.65 & --- & --- & --- \\
\hline 17 & --- & --- & --- & --- & --- & --- & --- & --- & 866.74 & 866.64 & -- & -- \\
\hline 18 & --- & --- & --- & --- & --- & --- & --- & --- & --- & --- & 866.28 & -- \\
\hline 19 & 866.53 & --- & --- & --- & --- & --- & --- & -- & --- & --- & -- & -- \\
\hline 20 & --- & --- & --- & --- & --- & --- & --- & --- & --- & --- & --- & -- \\
\hline 21 & --- & --- & --- & --- & --- & --- & --- & 866.82 & --- & -- & 866.36 & -- \\
\hline 22 & --- & --- & --- & --- & --- & --- & --- & --- & --- & --- & --- & -- \\
\hline 23 & 866.49 & --- & --- & --- & --- & --- & --- & --- & 866.84 & --- & --- & -- \\
\hline 24 & --- & 866.49 & --- & --- & -- & --- & --- & --- & --- & --- & --- & -- \\
\hline 25 & --- & -- & --- & --- & -- & --- & -- & -- & --- & --- & -- & 865.88 \\
\hline 26 & --- & --- & --- & --- & --- & --- & --- & 866.86 & --- & --- & 866.34 & --- \\
\hline 27 & --- & 866.49 & --- & --- & --- & --- & --- & --- & 866.78 & --- & --- & --- \\
\hline 28 & -- & -- & --- & --- & -- & --- & -- & -- & --- & --- & -- & 865.82 \\
\hline 29 & --- & --- & --- & --- & --- & --- & --- & --- & --- & 866.46 & --- & --- \\
\hline 30 & 866.41 & --- & --- & --- & --- & --- & --- & --- & --- & --- & --- & -- \\
\hline 31 & -- & --- & --- & --- & --- & --- & --- & 866.80 & --- & --- & --- & --- \\
\hline
\end{tabular}




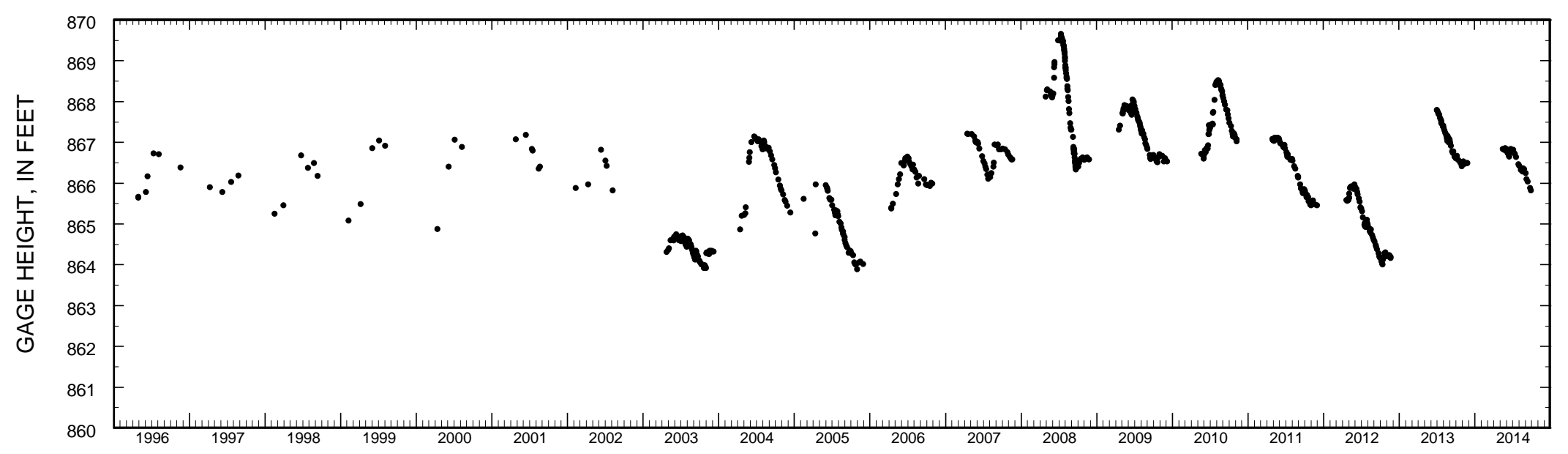

Stage hydrograph for Middle Genesee Lake, 1996-2014. 


\section{LAKE MONONA AT MADISON, WI}

LOCATION.--Lat 4303'48", long 89²3'49" referenced to North American Datum of 1927, in SE 1/4 SW 1/4 Sec.23, T.7 N., R.9 E., Dane County, WI, Hydrologic Unit 07090001, in Brittingham Park, in Madison.

SURFACE AREA.--5.3 mi².

DRAINAGE AREA.--279 $\mathrm{mi}^{2}$ of which $36.6 \mathrm{mi}^{2}$ may be noncontributing.

PERIOD OF RECORD.--September 1915 to current year (fragmentary) in reports of the Geological Survey. For 1856 to March 1917 in reports of Wisconsin Railroad Commission, volume 19.

REVISED RECORDS.--WSP 1338: Lake area. WDR WI-73-1: Drainage area.

GAGE.--Water-stage recorder. Datum of gage is $840.00 \mathrm{ft}$ above NGVD of 1929, or $5.60 \mathrm{ft}$ below City of Madison datum. Prior to Oct. 1, 1979, datum $843.61 \mathrm{ft}$; prior to Nov. 15, 1971, nonrecording gage at same site.

REMARKS.--Lake level regulated by concrete dam with four 12-foot stop-log sections and 12-foot lock at outlet of Lake Waubesa. Gage-height telemeter at station. EXTREMES FOR PERIOD OF RECORD.--Maximum gage height observed, $7.92 \mathrm{ft}$, June 15, 2008; minimum observed, $3.22 \mathrm{ft}$, Jan. 20, 1965, current datum.

EXTREMES FOR CURRENT YEAR.--Maximum recorded gage height, 6.28 ft, Aug. 26; minimum recorded, 4.13 ft, Apr. 12. 
05429000 LAKE MONONA AT MADISON, WI

GAGE HEIGHT, FEET

WATER YEAR OCTOBER 2013 TO SEPTEMBER 2014

DAILY MEAN VALUES

\begin{tabular}{|c|c|c|c|c|c|c|c|c|c|c|c|c|}
\hline DAY & OCT & NOV & DEC & JAN & FEB & MAR & APR & MAY & JUN & JUL & AUG & SEP \\
\hline 1 & 5.05 & 4.98 & 5.15 & 4.44 & 4.36 & --- & 4.37 & 5.21 & 5.21 & 6.24 & 5.73 & 6.14 \\
\hline 2 & 5.04 & 4.99 & 5.15 & 4.42 & 4.36 & --- & 4.35 & 5.19 & 5.46 & 6.27 & 5.76 & 6.13 \\
\hline 3 & 5.03 & 5.00 & 5.16 & 4.41 & 4.36 & --- & 4.34 & 5.16 & 5.54 & 6.24 & 5.78 & 6.08 \\
\hline 4 & 5.04 & 5.01 & 5.15 & 4.40 & 4.36 & --- & 4.31 & 5.13 & 5.55 & 6.21 & 5.85 & 6.17 \\
\hline 5 & 5.05 & 5.02 & 5.06 & 4.39 & 4.36 & -- & 4.26 & 5.13 & 5.53 & 6.18 & 5.98 & 6.21 \\
\hline 6 & 5.04 & 5.08 & 4.99 & 4.38 & 4.35 & -- & 4.24 & 5.11 & 5.49 & 6.16 & 5.98 & 6.17 \\
\hline 7 & 5.03 & 5.08 & 4.95 & 4.38 & 4.35 & -- & 4.21 & 5.09 & 5.46 & 6.12 & 5.98 & 6.13 \\
\hline 8 & 5.02 & 5.07 & 4.93 & 4.39 & 4.35 & -- & 4.19 & 5.06 & 5.44 & 6.11 & 5.98 & 6.10 \\
\hline 9 & 5.01 & 5.04 & 4.90 & 4.39 & 4.36 & 4.30 & 4.18 & 5.04 & 5.40 & 6.08 & 5.98 & 6.06 \\
\hline 10 & 5.01 & 5.02 & 4.86 & 4.40 & 4.36 & --- & 4.16 & 5.03 & 5.38 & 6.05 & 5.97 & 6.08 \\
\hline 11 & 5.00 & 5.01 & 4.83 & 4.42 & 4.36 & --- & 4.14 & 5.01 & 5.36 & 6.03 & 5.98 & 6.04 \\
\hline 12 & 4.99 & 4.98 & 4.79 & 4.41 & 4.37 & 4.36 & 4.13 & 5.09 & 5.32 & 6.01 & 5.98 & 6.00 \\
\hline 13 & 4.98 & 4.96 & 4.76 & 4.40 & 4.38 & 4.36 & 4.25 & 5.18 & 5.27 & 6.01 & 5.97 & 5.95 \\
\hline 14 & 4.99 & 4.96 & 4.73 & 4.40 & 4.38 & 4.37 & 4.60 & 5.17 & 5.27 & 5.98 & 5.97 & 5.90 \\
\hline 15 & 5.00 & 4.98 & 4.69 & 4.39 & 4.37 & 4.39 & 4.74 & 5.16 & 5.26 & 5.93 & 5.96 & 5.87 \\
\hline 16 & 5.00 & 5.01 & 4.67 & 4.38 & 4.37 & 4.40 & 4.85 & 5.14 & 5.25 & 5.92 & 5.96 & 5.84 \\
\hline 17 & 5.01 & 5.10 & 4.65 & 4.37 & 4.38 & 4.41 & 4.89 & 5.10 & 5.40 & 5.91 & 5.96 & 5.80 \\
\hline 18 & 5.00 & 5.14 & 4.62 & 4.36 & 4.39 & 4.40 & 4.90 & 5.07 & 5.47 & 5.92 & 5.94 & 5.77 \\
\hline 19 & 4.98 & 5.16 & 4.60 & 4.36 & 4.39 & 4.43 & 4.91 & 5.06 & 5.65 & 5.94 & 6.01 & 5.73 \\
\hline 20 & 4.98 & 5.19 & 4.58 & 4.35 & 4.42 & 4.43 & 4.92 & 5.03 & 5.88 & 5.96 & 6.00 & 5.70 \\
\hline 21 & 4.95 & 5.20 & 4.56 & 4.34 & 4.47 & 4.44 & 4.95 & 4.99 & 5.99 & 5.94 & 6.03 & 5.67 \\
\hline 22 & 4.94 & 5.18 & 4.57 & 4.34 & 4.45 & 4.43 & 4.95 & 4.95 & 6.00 & 5.90 & 6.07 & 5.62 \\
\hline 23 & 4.91 & 5.16 & 4.56 & 4.33 & 4.43 & 4.41 & 4.95 & 4.93 & 5.99 & 5.87 & 6.07 & 5.59 \\
\hline 24 & 4.88 & 5.14 & 4.54 & 4.33 & 4.41 & 4.40 & 4.99 & 4.91 & 5.97 & 5.83 & 6.07 & 5.56 \\
\hline 25 & 4.85 & 5.16 & 4.53 & 4.33 & 4.39 & 4.39 & 5.02 & 4.89 & 5.94 & 5.81 & 6.19 & 5.53 \\
\hline 26 & 4.83 & 5.15 & 4.51 & 4.34 & 4.38 & 4.38 & 5.03 & 4.88 & 5.90 & 5.80 & 6.28 & 5.51 \\
\hline 27 & 4.83 & 5.15 & 4.50 & 4.33 & 4.37 & 4.38 & 5.05 & 5.01 & 5.87 & 5.78 & 6.26 & 5.49 \\
\hline 28 & 4.85 & 5.16 & 4.48 & 4.33 & --- & 4.41 & 5.13 & 5.34 & 5.82 & 5.75 & 6.25 & 5.46 \\
\hline 29 & 4.84 & 5.16 & 4.46 & 4.33 & --- & 4.39 & 5.21 & 5.30 & 5.80 & 5.73 & 6.22 & 5.43 \\
\hline 30 & 4.84 & 5.16 & 4.45 & 4.35 & --- & 4.38 & 5.22 & 5.26 & 6.00 & 5.73 & 6.21 & 5.41 \\
\hline 31 & 4.90 & -- & 4.44 & 4.36 & -- & 4.38 & --- & 5.23 & --- & 5.72 & 6.18 & --- \\
\hline MEAN & 4.96 & 5.08 & 4.74 & 4.37 & --- & --- & 4.65 & 5.09 & 5.60 & 5.97 & 6.02 & 5.84 \\
\hline MAX & 5.05 & 5.20 & 5.16 & 4.44 & --- & -- & 5.22 & 5.34 & 6.00 & 6.27 & 6.28 & 6.21 \\
\hline MIN & 4.83 & 4.96 & 4.44 & 4.33 & --- & --. & 4.13 & 4.88 & 5.21 & 5.72 & 5.73 & 5.41 \\
\hline
\end{tabular}




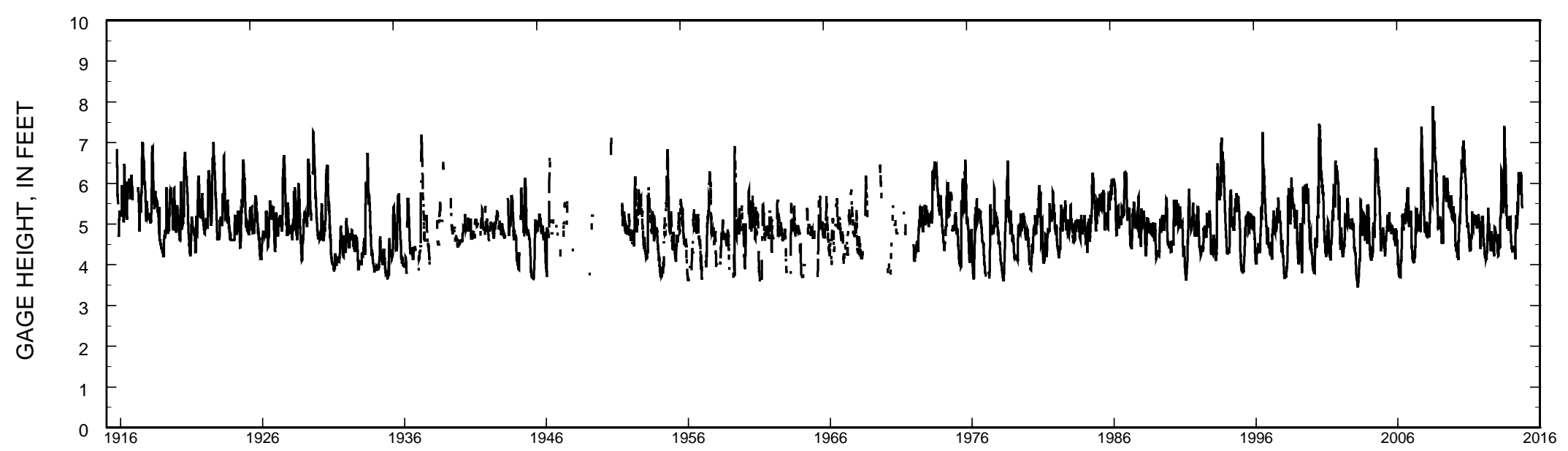

Stage hydrograph for Lake Monona, 1915-2014. 


\section{NORTH LAKE NEAR ELKHORN, WI}

LOCATION.--Lat 4244'38", long 88³7'45" referenced to North American Datum of 1927, Walworth County, WI, Hydrologic Unit 07120006.

DRAINAGE AREA.--10.80 $\mathrm{mi}^{2}$ of which $10.79 \mathrm{mi}^{2}$ probably is noncontributing.

PERIOD OF RECORD.--May 1937 to current year.

GAGE.--A staff gage is periodically read by an observer, and reported in local datum.

COOPERATION.--Wisconsin Department of Natural Resources.

EXTREMES FOR PERIOD OF RECORD.--Maximum observed gage height, $15.62 \mathrm{ft}$, June 22, 1974; miminum observed gage height, $5.81 \mathrm{ft}$, Dec. 1, 1958.

EXTREMES FOR CURRENT YEAR.--Maximum observed gage height, $12.10 \mathrm{ft}$, May 14; miminum observed gage height, $11.26 \mathrm{ft}$,

Sept. 30.

GAGE HEIGHT, FEET

WATER YEAR OCTOBER 2013 TO SEPTEMBER 2014

\begin{tabular}{|c|c|c|c|c|c|c|c|c|c|c|c|c|}
\hline DAY & OCT & NOV & DEC & JAN & FEB & MAR & APR & MAY & JUN & JUL & AUG & SEP \\
\hline 1 & 11.93 & --- & -- & --- & --- & --- & --- & --- & --- & -- & --- & --- \\
\hline 2 & --- & --- & --- & --- & --- & --- & --- & --- & --- & --- & --- & -- \\
\hline 3 & --- & --- & --- & --- & --- & --- & --- & --- & 11.86 & --- & --- & 11.40 \\
\hline 4 & --- & --- & --- & --- & --- & --- & --- & --. & --- & 11.82 & --. & --- \\
\hline 5 & --- & -- & --- & --- & --- & --- & -- & --- & --- & --- & --- & --- \\
\hline 6 & --- & -- & --- & --- & --- & --- & --- & -- & --- & --- & 11.54 & --- \\
\hline 7 & --- & --- & --- & --- & --- & --- & --- & --- & --- & --- & --- & --- \\
\hline 8 & 11.98 & --- & --- & --- & --- & --. & --- & --- & --- & --- & --- & -- \\
\hline 9 & -- & -- & --- & --- & --- & -- & -- & -- & --- & -- & -- & -- \\
\hline 10 & --. & --- & --- & --. & --- & --- & --- & --- & --- & 11.76 & --- & 11.42 \\
\hline 11 & --- & -- & --- & --- & --- & --- & -- & --- & --- & --- & --- & --- \\
\hline 12 & --- & --- & --- & --- & --- & --- & --- & --- & 11.82 & --- & --- & -- \\
\hline 13 & --- & --- & --- & --- & --- & --- & --- & --- & --- & --- & --- & -- \\
\hline 14 & --- & --- & -- & -- & --- & -- & --- & 12.10 & -- & -- & --- & -- \\
\hline 15 & --- & --- & --- & --- & --- & --- & --- & --- & -- & -- & --- & --- \\
\hline 16 & -- & -- & --- & --- & -- & --- & -- & -- & --- & --- & -- & 11.36 \\
\hline 17 & --- & --- & --- & --- & --- & --- & --- & 12.06 & --- & --- & --- & -- \\
\hline 18 & 11.97 & --- & --- & --- & --- & -- & --- & --- & --- & 11.78 & 11.36 & -- \\
\hline 19 & -- & -- & -- & --- & -- & -- & -- & -- & -- & -- & -- & -- \\
\hline 20 & --- & --- & --- & --- & --- & --- & --- & --- & --- & --- & --- & --- \\
\hline 21 & -- & --- & -- & -- & --- & -- & -- & 12.00 & -- & -- & --- & -- \\
\hline 22 & 11.96 & --- & --- & --- & --- & --- & --- & --- & --- & --- & --- & --- \\
\hline 23 & -- & --- & --- & --- & --- & --- & --- & --- & -- & 11.60 & --- & 11.34 \\
\hline 24 & -- & -- & --- & -- & --- & -- & -- & 11.96 & -- & -- & -- & -- \\
\hline 25 & --- & --- & --- & --- & -- & -- & --- & -- & 11.90 & --- & -- & --- \\
\hline 26 & --- & --- & --- & --- & --- & --- & --- & --- & --- & --- & --- & --- \\
\hline 27 & -- & --- & -- & --- & --- & --- & --- & --- & -- & -- & --- & --- \\
\hline 28 & --- & -- & --- & --- & -- & -- & --- & -- & --- & --- & -- & -- \\
\hline 29 & -- & -- & --- & --- & -- & -- & -- & 11.96 & --- & --- & --- & -- \\
\hline 30 & --- & --- & --- & --- & --- & --- & --- & --- & --- & 11.56 & --- & 11.26 \\
\hline 31 & --- & -- & --- & --- & --- & --- & --- & --- & --- & --- & --- & --- \\
\hline
\end{tabular}




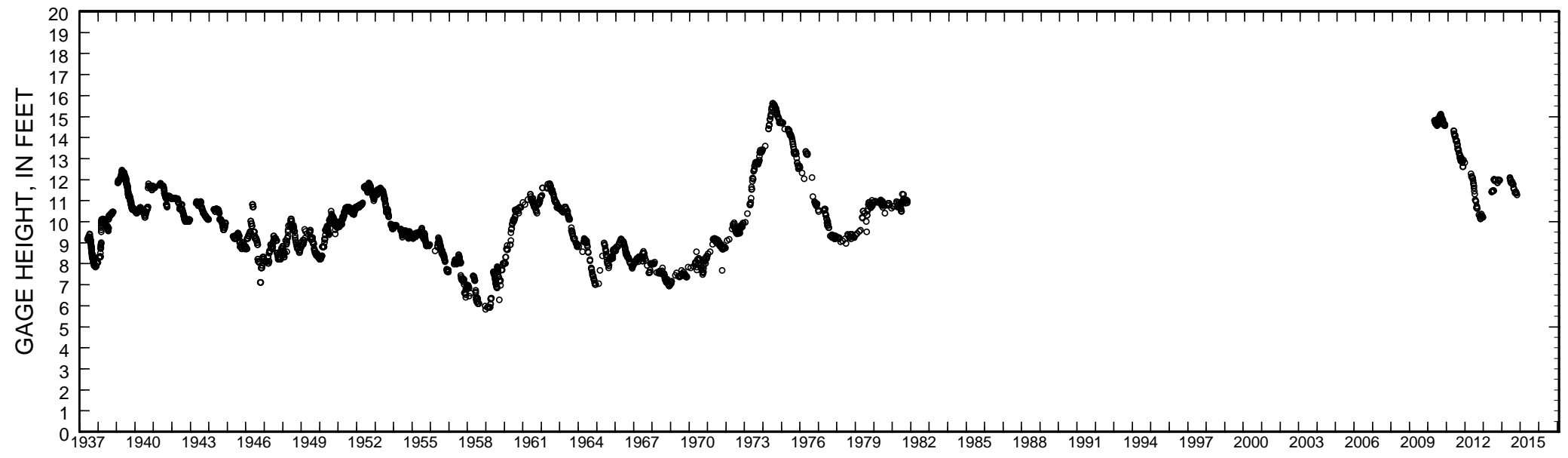

Stage hydrograph for North Lake, 1937-2014. 
LOCATION.--Lat 4305'51", long 88²7'35", in NW 1/4 SE 1/4 Sec.2, T.7 N., R.17 E., Waukesha County, Hydrologic Unit 07090001, at Oconomowoc. SURFACE AREA.--1.20 $\mathrm{mi}^{2}$.

PERIOD OF RECORD.--March 1986 to current year.

REMARKS.--Lake sampled near center at the deep hole. Water-quality analyses done by Wisconsin State Laboratory of Hygiene.

WATER-QUALITY DATA, MARCH 6 TO AUGUST 22, 2014

(Milligrams per liter unless otherwise indicated)

\begin{tabular}{|c|c|c|c|c|c|c|c|c|c|c|c|c|c|}
\hline Date & $\begin{array}{c}\text { Trans- } \\
\text { parency } \\
\text { Secchi } \\
\text { disc, } \\
\text { meters } \\
(\odot \odot \odot 78)\end{array}$ & $\begin{array}{c}\text { Sam- } \\
\text { pling } \\
\text { depth, } \\
\text { meters } \\
(\odot 0098)\end{array}$ & $\begin{array}{c}\text { Temper - } \\
\text { ature, } \\
\text { water, } \\
\text { deg C } \\
(00010)\end{array}$ & $\begin{array}{c}\text { Specif- } \\
\text { ic } \\
\text { conduc- } \\
\text { tance, } \\
\text { wat unf } \\
\text { us/cm @ } \\
25 \text { degc } \\
(0 \odot \odot 95)\end{array}$ & $\begin{array}{c}\text { pH, } \\
\text { water, } \\
\text { unfltrd } \\
\text { field, } \\
\text { std } \\
\text { units } \\
(\odot \odot 40 \odot)\end{array}$ & $\begin{array}{c}\text { Dis- } \\
\text { solved } \\
\text { oxygen, } \\
\text { mg/L } \\
(00300)\end{array}$ & $\begin{array}{l}\text { Chloro- } \\
\text { phyll a } \\
\text { trichro } \\
\text {-matic } \\
\text { method, } \\
\text { uncorr, } \\
\text { ug/L } \\
(32210)\end{array}$ & $\begin{array}{c}\text { Phos- } \\
\text { phorus, } \\
\text { water, } \\
\text { unfltrd } \\
\text { mg/L } \\
\text { as } P \\
(00665)\end{array}$ & $\begin{array}{l}\text { Ortho- } \\
\text { phos- } \\
\text { phate, } \\
\text { water, } \\
\text { fltrd, } \\
\text { mg/L } \\
\text { as P } \\
(00671)\end{array}$ & $\begin{array}{c}\text { Total } \\
\text { nitro- } \\
\text { gen, } \\
\text { water, } \\
\text { unfltrd } \\
\text { mg/L } \\
(00600)\end{array}$ & $\begin{array}{c}\text { Ammonia } \\
\text { water, } \\
\text { fltrd, } \\
\text { mg/L } \\
\text { as N } \\
(00608)\end{array}$ & $\begin{array}{c}\text { Ammonia } \\
+ \\
\text { org-N, } \\
\text { water, } \\
\text { unfltrd } \\
\text { mg/L } \\
\text { as N } \\
(00625)\end{array}$ & $\begin{array}{c}\text { Nitrate } \\
+ \\
\text { nitrite } \\
\text { water, } \\
\text { fltrd, } \\
\text { mg/L } \\
\text { as N } \\
(00631)\end{array}$ \\
\hline
\end{tabular}

\begin{tabular}{|c|c|c|c|c|c|c|c|c|c|c|c|c|c|}
\hline \multicolumn{14}{|l|}{ MAR 2014} \\
\hline 06... & -- & 1.0 & 1.9 & 628 & 8.1 & 11.0 & 0.388 & 0.014 & -- & - & -- & -- & - - \\
\hline $06 \ldots$ & -- & 18.0 & 3.6 & 668 & 8.0 & 0.8 & -- & 0.017 & -- & - & - - & -- & -- \\
\hline \multicolumn{14}{|l|}{ MAY } \\
\hline $20 \ldots$ & 6.45 & - & - - & -- & -- & -- & - & -- & -- & - & - & -- & -- \\
\hline $20 \ldots$ & -- & 0.50 & 15.1 & 596 & 8.4 & 12.8 & 1.09 & $\odot .009$ & $<0.002$ & 0.82 & 0.034 & 0.57 & 0.256 \\
\hline \multicolumn{14}{|l|}{ JUL } \\
\hline $01 \ldots$ & 5.45 & - & -- & - - & -- & -- & - & -- & -- & - & - & -- & -- \\
\hline $01 \ldots$ & - - & 0.50 & 24.3 & 569 & 8.7 & 9.4 & 2.75 & 0.012 & -- & - & - & -- & -- \\
\hline $01 \ldots$ & -- & 18.5 & 8.8 & 616 & 7.8 & 3.7 & -- & 0.010 & -- & - & - - & - & -- \\
\hline $24 \ldots$ & 2.55 & -- & -- & -- & - - & - - & -- & -- & -- & - & - - & - & -- \\
\hline $24 \ldots$ & -- & 18.5 & 8.3 & 631 & 7.6 & 0.8 & - - & $\odot .029$ & -- & - & - & -- & -- \\
\hline \multicolumn{14}{|l|}{$A U G$} \\
\hline $22 \ldots$ & 4.30 & -- & -- & - - & -- & -- & -- & -- & -- & -- & -- & -- & - - \\
\hline $22 \ldots$ & - - & 0.50 & 25.0 & 555 & 8.7 & 8.9 & 1.37 & 0.011 & -- & -- & -- & -- & -- \\
\hline $22 \ldots$ & -- & 18.5 & 8.2 & 645 & 7.6 & 0.3 & -- & 0.028 & - - & -- & -- & -- & - - \\
\hline
\end{tabular}




\section{OCONOMOWOC LAKE NO. 1 (CENTER) AT OCONOMOWOC, WI}

WATER-QUALITY DATA, MARCH 6 TO AUGUST 22, 2014

(Milligrams per liter unless otherwise indicated)

Date



MAR 2014
$06 \ldots$
$06 \ldots$
MAY
$20 \ldots$
$20 \ldots$
JUL
$01 \ldots$
$01 \ldots$
$01 \ldots$
$24 \ldots$
$24 \ldots$
$24 \ldots$
AUG
$22 \ldots$
$22 \ldots$
$22 \ldots$

Turbdty Appar-

white ent

light, color, Hard-

det ang water, ness,

ness, Calcium

ANC,

Magnes- Potas- $\begin{gathered}\text { wat unf } \\ \text { fixed }\end{gathered}$

degrees

water,

Sodium,

Chlor -

Chlor- Silica,
ide, sulfate water,

Manga-

water, water,

water,

water

water,

ltrd,

nese,

$\begin{array}{ccc}\text { NTU } & \text { units } & \text { CaC03 } \\ (63675) & (00081) & (0 \odot 9 \odot \odot)\end{array}$

$\mathrm{mg} / \mathrm{L} \quad \mathrm{mg} / \mathrm{L} \quad \mathrm{mg} / \mathrm{L}$

fltrd, $\mathrm{mg} / \mathrm{L}$ as

fltrd,

ltrd,

g/L as

water, water

(००925) (००930)

$(00935) \quad(00417)$
0

$\begin{array}{lll}(00940) & (00945) & (00955)\end{array}$ (01046) (01056)

\footnotetext{
$06 \ldots$

$20 \ldots$
$20 \ldots$

UL

$01 \ldots$

$01 \ldots$

...

$22 \ldots$
}

$\begin{array}{cc}-- & -- \\ -- & - \\ -- & - \\ 0.6 & 15 \\ -- & -- \\ -- & -- \\ -- & -- \\ -- & - \\ -- & - \\ -- & -- \\ & \\ -- & -- \\ -- & -- \\ -- & -- \\ & \end{array}$

--
--
--
254
--
--
--
--
--
--
--
--
--

$$
--
$$$$
\begin{array}{ll}
-- & - \\
-- & -
\end{array}
$$$$
-
$$$$
32.4
$$$$
22.5
$$$$
2 . \odot 3 \quad 225
$$

$$
--
$$

$$
-
$$

Dis -

solved

solids

dried @

180degC

Date

wa/

(70300)

MAY

$\begin{array}{lr}20 \ldots & -- \\ 20 . . & 336\end{array}$ 
430551088273500 OCONOMOWOC LAKE NO. 1 (CENTER) AT OCONOMOWOC, WI

LAKE-DEPTH PROFILES, MARCH 6 TO AUGUST 22, 2014

03-06-14

05-20-14

07-01-14

07-24-14

$08-22-14$
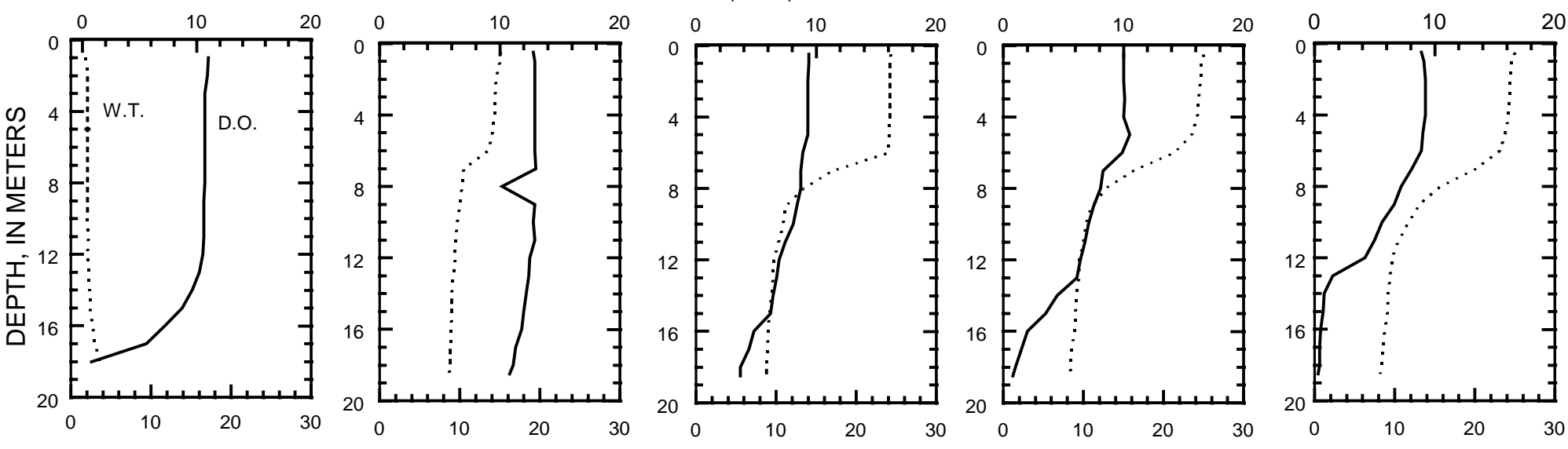

WATER TEMPERATURE (W.T.), IN DEGREES CELSIUS
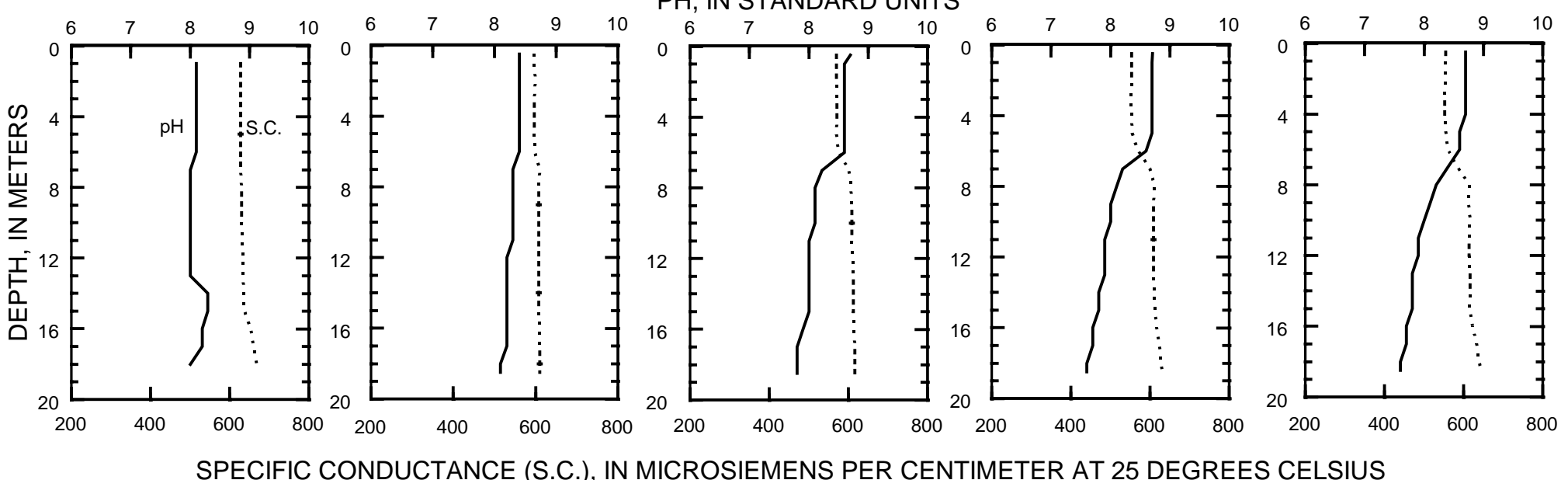

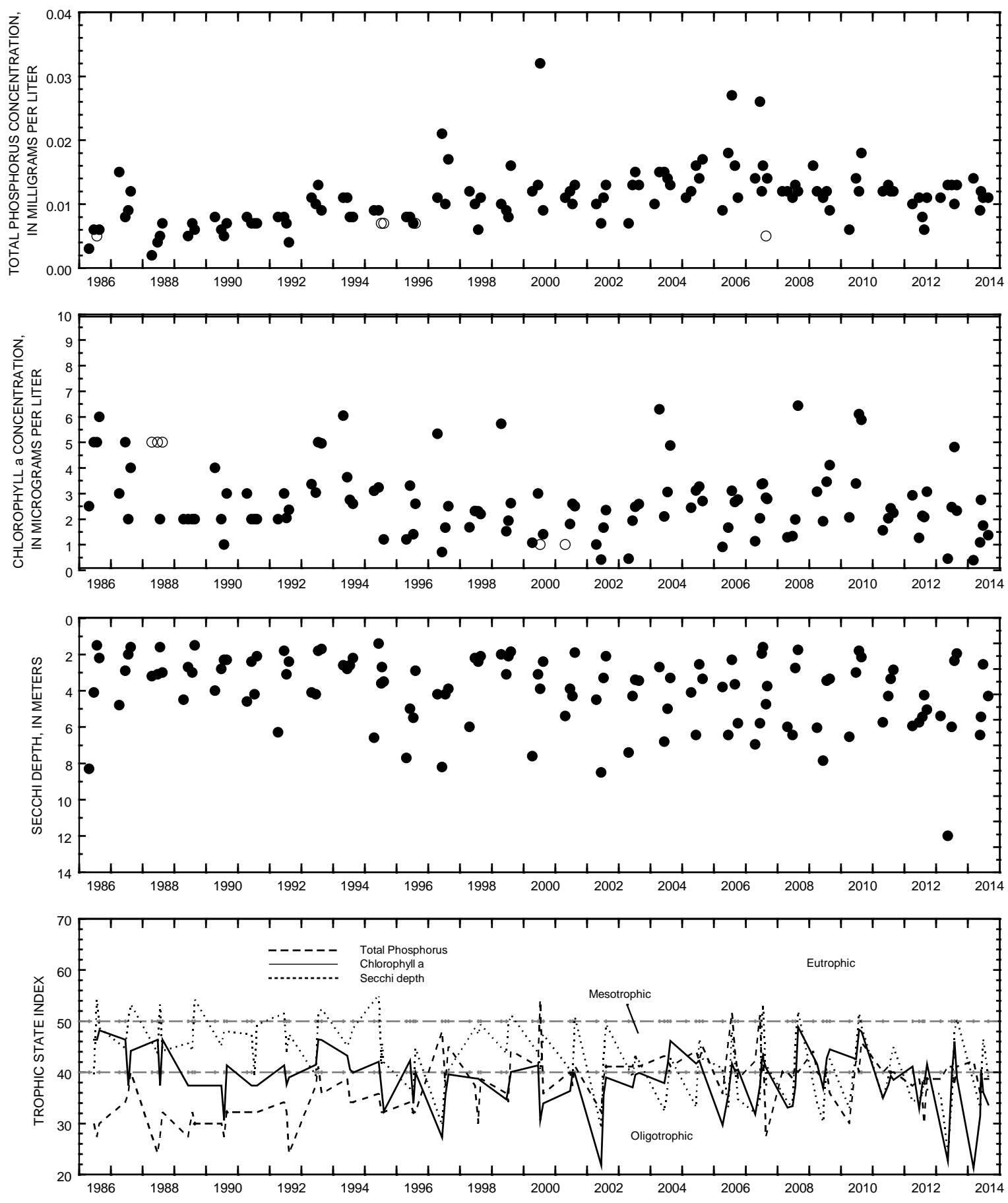

Surface total phosphorus, chlorophyll a concentrations, Secchi depths, and TSI data for Oconomowoc Lake, Center Site, at Oconomowoc, Wisconsin. (Open circles on the first two plots indicate laboratory detection limit for selected analyses. Actual concentrations for these particular analyses are less than the plotted circles.) 


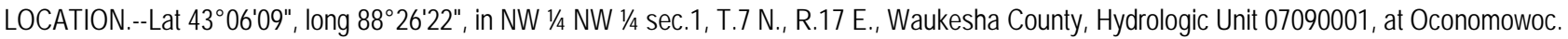
SURFACE AREA.-1.20 $\mathrm{mi}^{2}$

PERIOD OF RECORD.--March 1986 to current year.

REMARKS.--Lake sampled at the deepest point in northeast bay near Hewitt Point. Water-quality analyses done by Wisconsin State Laboratory of Hygiene.

\begin{tabular}{|c|c|c|c|c|c|c|c|c|}
\hline \multirow[b]{2}{*}{ Date } & \multirow[b]{2}{*}{$\begin{array}{c}\text { Trans- } \\
\text { parency } \\
\text { Secchi } \\
\text { disc, } \\
\text { meters } \\
(00078)\end{array}$} & \multirow[b]{2}{*}{$\begin{array}{c}\text { Sam- } \\
\text { pling } \\
\text { depth, } \\
\text { meters } \\
(00098)\end{array}$} & \multicolumn{6}{|c|}{$\begin{array}{l}\text { WATER-QUALITY DATA, MARCH } 6 \text { TO AUGUST 22, } \\
\text { (Milligrams per liter unless otherwise indicated) }\end{array}$} \\
\hline & & & $\begin{array}{c}\text { Temper- } \\
\text { ature, } \\
\text { water, } \\
\text { deg C } \\
(00010)\end{array}$ & $\begin{array}{c}\text { Specif- } \\
\text { ic } \\
\text { conduc- } \\
\text { tance, } \\
\text { wat unf } \\
\text { uS/cm @ } \\
25 \text { degc } \\
(00095)\end{array}$ & $\begin{array}{c}\text { pH, } \\
\text { water, } \\
\text { unfltrd } \\
\text { field, } \\
\text { std } \\
\text { units } \\
(0040 \odot)\end{array}$ & $\begin{array}{c}\text { Dis- } \\
\text { solved } \\
\text { oxygen, } \\
\text { mg/L } \\
(00300)\end{array}$ & $\begin{array}{l}\text { Chloro- } \\
\text { phyll a } \\
\text { trichro } \\
\text { - matic } \\
\text { method, } \\
\text { uncorr, } \\
\text { ug/L } \\
(32210)\end{array}$ & $\begin{array}{c}\text { Phos- } \\
\text { phorus, } \\
\text { water, } \\
\text { unfltrd } \\
\mathrm{mg} / \mathrm{L} \\
\text { as } \mathrm{P} \\
(00665)\end{array}$ \\
\hline \multicolumn{9}{|c|}{ MAR 2014} \\
\hline $06 \ldots$ & -- & 1.0 & 2.5 & 685 & 8.0 & 10.0 & 0.754 & 0.010 \\
\hline $06 \ldots$ & - - & 14.0 & 4.1 & 759 & 7.6 & 2.6 & - - & 0.019 \\
\hline \multicolumn{9}{|l|}{ MAY } \\
\hline $20 \ldots$ & 10.2 & - - & - - & - - & - - & - - & - - & - - \\
\hline $20 \ldots$ & - - & 0.50 & 16.4 & 647 & 8.4 & 13.1 & 0.848 & 0.008 \\
\hline \multicolumn{9}{|l|}{ JUL } \\
\hline $01 \ldots$ & 6.45 & - - & - - & - - & -- & - - & -- & - - \\
\hline $01 \ldots$ & - - & $\odot .5 \odot$ & 24.6 & 613 & 8.5 & 9.0 & 1.01 & 0.009 \\
\hline $01 \ldots$ & -- & 14.5 & 8.8 & 678 & 7.8 & 6.9 & -- & 0.017 \\
\hline $24 \ldots$ & 3.45 & - - & - - & - - & - - & - - & - - & - - \\
\hline $24 \ldots$ & - - & $\odot .50$ & 25.5 & 605 & 8.6 & 9.9 & 1.28 & 0.010 \\
\hline $24 \ldots$ & -- & 14.5 & 9.0 & 685 & 7.6 & 2.6 & -- & $\odot .017$ \\
\hline \multicolumn{9}{|l|}{ AUG } \\
\hline $22 \ldots$ & 5.30 & -- & -- & - - & -- & - - & - - & -- \\
\hline $22 \ldots$ & - - & 0.50 & 25.5 & 582 & 8.4 & 8.8 & 1.31 & 0.011 \\
\hline $22 \ldots$ & - - & 14.5 & 9.0 & 707 & 7.6 & 0.6 & -- & 0.044 \\
\hline
\end{tabular}


430609088262200 OCONOMOWOC LAKE NO. 2 (OFF HEWITT POINT) AT OCONOMOWOC, WI

LAKE-DEPTH PROFILES, MARCH 6 TO AUGUST 22, 2014

03-06-14

05-20-14

07-01-14

07-24-14

08-22-14

DISSOLVED OXYGEN (D.O.), IN MILLIGRAMS PER LITER
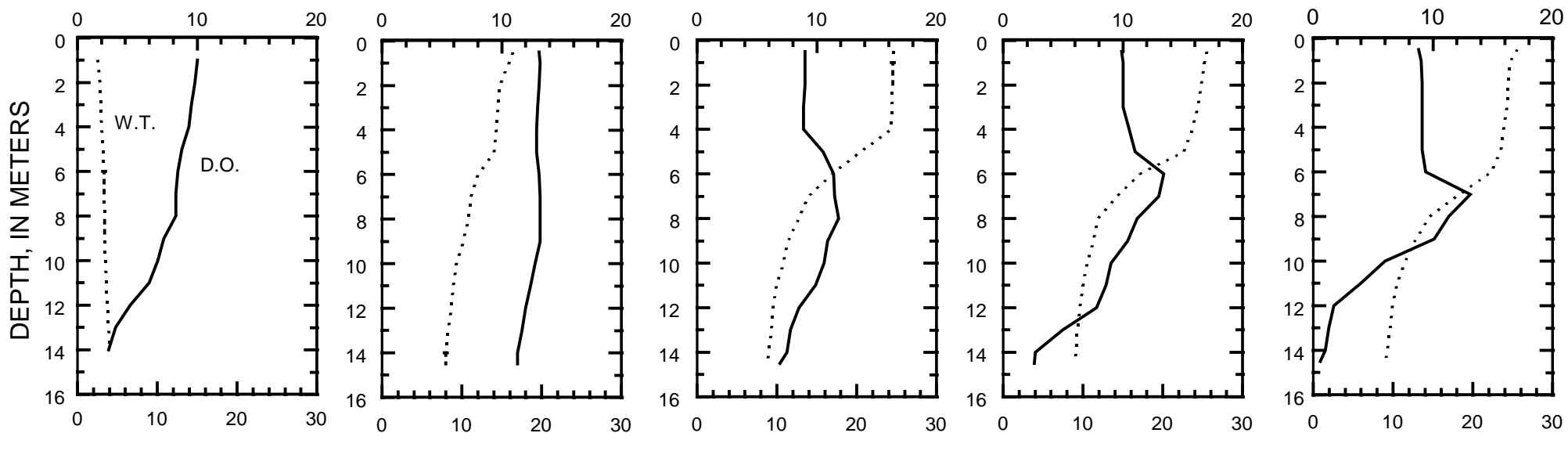

WATER TEMPERATURE (W.T.), IN DEGREES CELSIUS

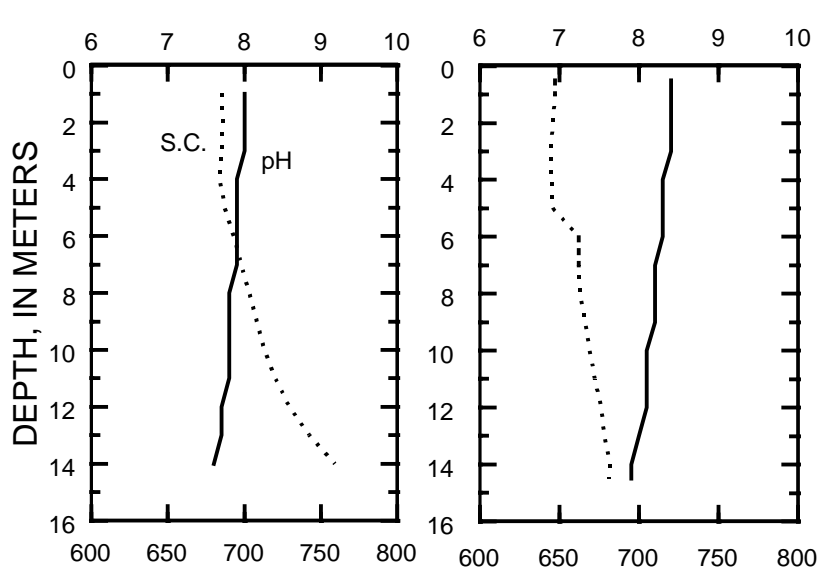

$\mathrm{PH}$, IN STANDARD UNITS
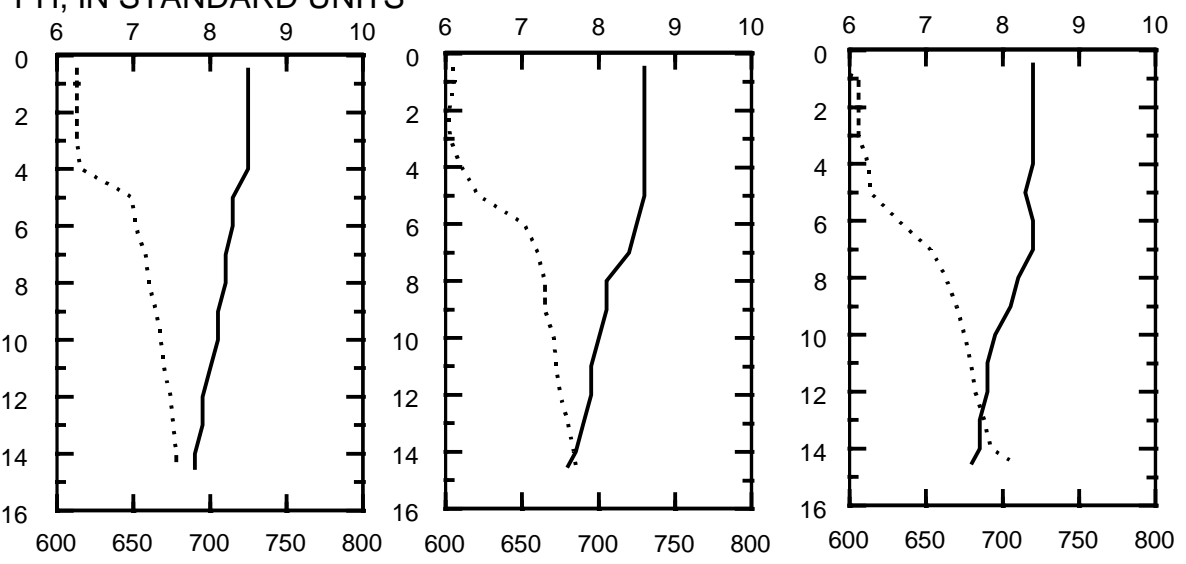

SPECIFIC CONDUCTANCE (S.C.), IN MICROSIEMENS PER CENTIMETER AT 25 DEGREES CELSIUS 

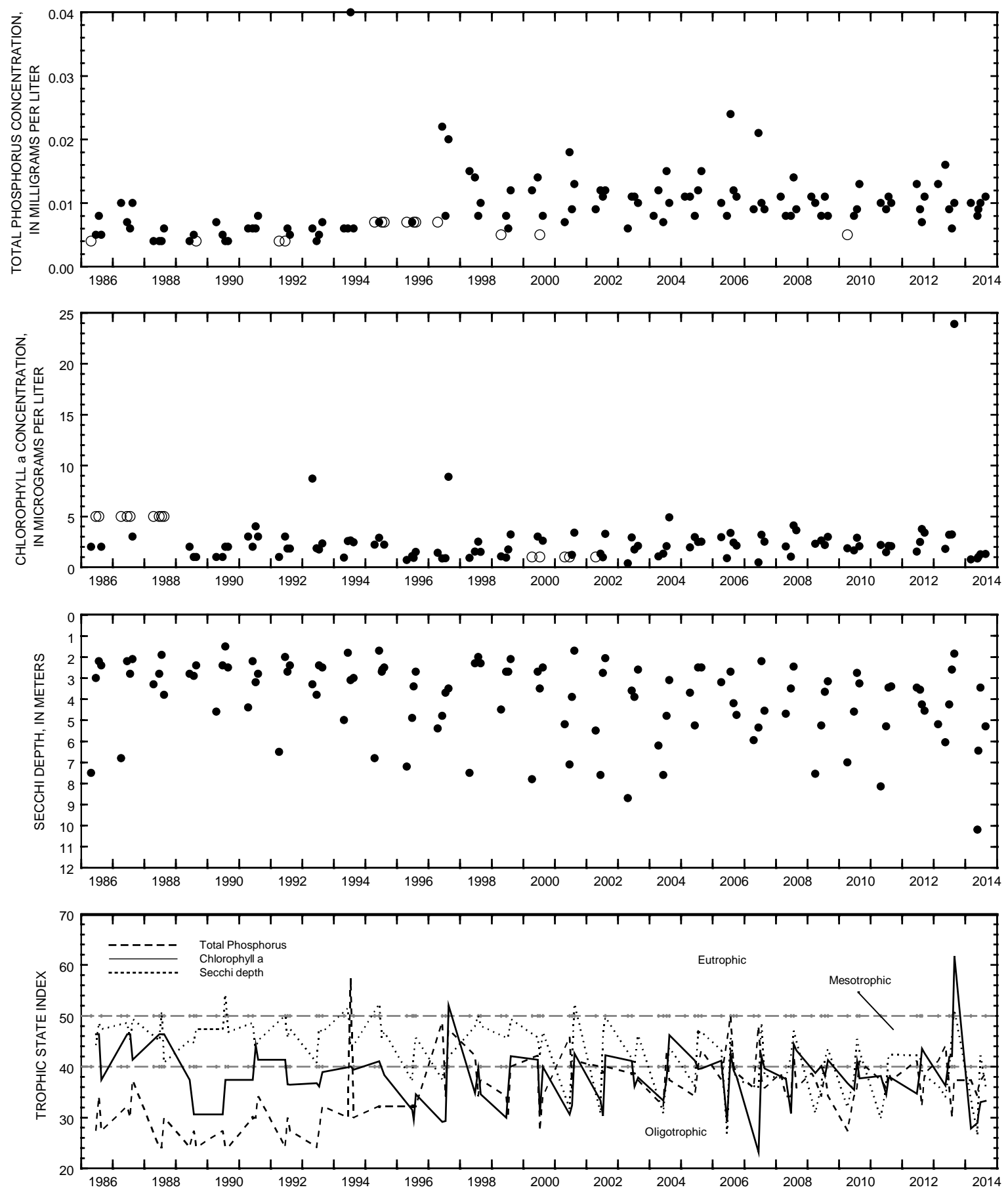

Surface total phosphorus, chlorophyll a concentrations, Secchi depths, and TSI data for Oconomowoc Lake, Hewitt Point, at Oconomowoc, Wisconsin.

(Open circles on the first two plots indicate laboratory detection limit for selected analyses. Actual concentrations for these particular analyses are less than the plotted circles.) 
430723088252100 OKAUCHEE LAKE AT OKAUCHEE, WI

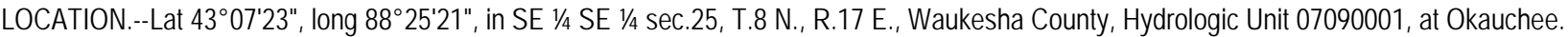

DRAINAGE AREA.--80.7 mi²

PERIOD OF RECORD.--February 1984 to September 2006, April to August 2008, April to August 2010, April to August 2012, March to August 2014

REMARKS.--Lake sampled near center at the deep hole. The lake was not sampled during winter ice cover because of unsafe winter conditions. Water-quality analyses done by Wisconsin State Laboratory of Hygiene.

WATER-QUALITY DATA, MARCH 6 TO AUGUST 22, 2014

(Milligrams per liter unless otherwise indicated)

\begin{tabular}{|c|c|c|c|c|c|c|c|c|c|c|c|c|c|}
\hline Date & $\begin{array}{c}\text { Trans- } \\
\text { parency } \\
\text { Secchi } \\
\text { disc, } \\
\text { meters } \\
(00 \odot 78)\end{array}$ & $\begin{array}{c}\text { Sam- } \\
\text { pling } \\
\text { depth, } \\
\text { meters } \\
(\odot \odot \odot 98)\end{array}$ & $\begin{array}{c}\text { Temper - } \\
\text { ature, } \\
\text { water, } \\
\text { deg C } \\
(00010)\end{array}$ & $\begin{array}{c}\text { Specif- } \\
\text { ic } \\
\text { conduc- } \\
\text { tance, } \\
\text { wat unf } \\
\text { uS/cm @ } \\
25 \text { degc } \\
(00095)\end{array}$ & $\begin{array}{c}\text { pH, } \\
\text { water, } \\
\text { unfltrd } \\
\text { field, } \\
\text { std } \\
\text { units } \\
(00400)\end{array}$ & $\begin{array}{c}\text { Dis- } \\
\text { solved } \\
\text { oxygen, } \\
\text { mg/L } \\
(00300)\end{array}$ & $\begin{array}{c}\text { Chloro- } \\
\text { phyll a } \\
\text { trichro } \\
\text {-matic } \\
\text { method, } \\
\text { uncorr, } \\
\text { ug/L } \\
(32210)\end{array}$ & $\begin{array}{c}\text { Phos- } \\
\text { phorus, } \\
\text { water, } \\
\text { unfltrd } \\
\text { mg/L } \\
\text { as P } \\
(00665)\end{array}$ & $\begin{array}{l}\text { Ortho- } \\
\text { phos- } \\
\text { phate, } \\
\text { water, } \\
\text { fltrd, } \\
\text { mg/L } \\
\text { as P } \\
(00671)\end{array}$ & $\begin{array}{c}\text { Total } \\
\text { nitro- } \\
\text { gen, } \\
\text { water, } \\
\text { unfltrd } \\
\text { mg/L } \\
(00600)\end{array}$ & $\begin{array}{c}\text { Ammonia } \\
\text { water, } \\
\text { fltrd, } \\
\text { mg/L } \\
\text { as N } \\
(\odot \odot 6 \odot 8)\end{array}$ & $\begin{array}{c}\text { Ammonia } \\
+ \\
\text { org-N, } \\
\text { water, } \\
\text { unfltrd } \\
\text { mg/L } \\
\text { as N } \\
(00625)\end{array}$ & $\begin{array}{c}\text { Nitrate } \\
+ \\
\text { nitrite } \\
\text { water, } \\
\text { fltrd, } \\
\text { mg/L } \\
\text { as N } \\
(00631)\end{array}$ \\
\hline \multicolumn{14}{|l|}{ MAR 2014} \\
\hline $06 \ldots$ & -- & 1.0 & 1.6 & 626 & 8.1 & 11.3 & 18.3 & $\odot .026$ & - & -- & - & -- & -- \\
\hline \multicolumn{14}{|l|}{ MAY } \\
\hline $20 \ldots$ & 2.65 & - - & -- & -- & - & - - & -- & -- & -- & - & - & -- & - \\
\hline $20 \ldots$ & -- & 0.50 & 15.0 & 602 & 8.4 & 14.1 & 4.57 & 0.013 & $<0.002$ & 0.96 & $<0.015$ & 0.60 & $\odot .354$ \\
\hline \multicolumn{14}{|l|}{ JUN } \\
\hline $16 \ldots$ & 5.00 & - - & - - & - - & - - & - - & - - & - - & - - & - - & - - & - - & - - \\
\hline $16 \ldots$ & -- & 0.50 & 22.0 & 593 & 8.4 & 8.9 & 3.37 & 0.017 & -- & - - & - - & 0.61 & - - \\
\hline $16 \ldots$ & -- & 27.0 & 6.9 & 618 & 7.6 & 1.2 & -- & 0.062 & -- & - - & -- & 1.0 & -- \\
\hline \multicolumn{14}{|l|}{ JUL } \\
\hline $24 \ldots$ & 2.85 & - - & - - & -- & - - & - - & - - & -- & - - & -- & -- & - - & - - \\
\hline $24 \ldots$ & - - & 0.50 & 24.5 & 561 & 8.7 & 9.9 & 6.17 & 0.016 & -- & - & - & -- & -- \\
\hline $22 \ldots$ & 3.80 & - - & - - & - - & - - & - - & -- & - - & - - & - - & - - & - - & -- \\
\hline $22 \ldots$ & -- & 0.50 & 24.5 & 544 & 8.7 & 9.3 & 3.51 & 0.011 & -- & -- & -- & -- & -- \\
\hline $22 \ldots$ & -- & 27.0 & 7.1 & 616 & 7.6 & 0.4 & - - & 0.025 & -- & -- & -- & -- & -- \\
\hline
\end{tabular}


430723088252100 OKAUCHEE LAKE AT OKAUCHEE, WI

WATER-QUALITY DATA, MARCH 6 TO AUGUST 22, 2014

(Milligrams per liter unless otherwise indicated)

\begin{tabular}{|c|c|c|c|c|c|c|c|c|c|c|c|c|c|}
\hline & Turbdty & $\begin{array}{l}\text { Appar - } \\
\text { ent }\end{array}$ & & & & & & ANC, & & & & & \\
\hline ate & $\begin{array}{c}\text { light, } \\
\text { det ang } \\
90+/-30 \\
\text { degrees } \\
\text { NTU } \\
(63675)\end{array}$ & $\begin{array}{c}\text { color, } \\
\text { water, } \\
\text { unfltrd } \\
\text { Pt-Co } \\
\text { units } \\
(00081)\end{array}$ & $\begin{array}{c}\text { Hard- } \\
\text { ness, } \\
\text { water, } \\
\text { mg/L as } \\
\text { CaC03 } \\
(0090 \odot)\end{array}$ & $\begin{array}{c}\text { Calcium } \\
\text { water, } \\
\text { fltrd, } \\
\text { mg/L } \\
(00915)\end{array}$ & $\begin{array}{c}\text { Magnes- } \\
\text { ium, } \\
\text { water, } \\
\text { fltrd, } \\
\text { mg/L } \\
(00925)\end{array}$ & $\begin{array}{c}\text { Sodium, } \\
\text { water, } \\
\text { fltrd, } \\
\text { mg/L } \\
(\odot \odot 930)\end{array}$ & $\begin{array}{l}\text { Potas- } \\
\text { sium, } \\
\text { water, } \\
\text { fltrd, } \\
\text { mg/L } \\
(00935)\end{array}$ & $\begin{array}{c}\text { fixed } \\
\text { end pt, } \\
\text { lab, } \\
\mathrm{mg} / \mathrm{L} \text { as } \\
\text { CaC03 } \\
(0 \odot 417)\end{array}$ & $\begin{array}{c}\text { Chlor - } \\
\text { ide, } \\
\text { water, } \\
\text { fltrd, } \\
\text { mg/L } \\
(0094 \odot)\end{array}$ & $\begin{array}{c}\text { Sulfate } \\
\text { water, } \\
\text { fltrd, } \\
\text { mg/L } \\
(00945)\end{array}$ & $\begin{array}{c}\text { Silica, } \\
\text { water, } \\
\text { fltrd, } \\
\text { mg/L as } \\
\text { Si02 } \\
(00955)\end{array}$ & $\begin{array}{c}\text { Iron, } \\
\text { water, } \\
\text { fltrd, } \\
\text { ug/L } \\
(01046)\end{array}$ & $\begin{array}{c}\text { Manga- } \\
\text { nese, } \\
\text { water, } \\
\text { fltrd, } \\
\text { ug/L } \\
(01056)\end{array}$ \\
\hline
\end{tabular}

MAR 2014

06...

$06 .$.

MAY

$20 \ldots$
$20 \ldots$

JUN

$16 \ldots$

$16 \ldots$ JUL

24 ...

$24 \ldots$

AUG

22 . .

$22 \ldots$

22 ...

$$
\begin{gathered}
-- \\
-- \\
-- \\
-- \\
-- \\
-- \\
-- \\
-- \\
\text { Dis- } \\
\text { solved } \\
\text { solids } \\
\text { dried @ } \\
\text { 180degC } \\
\text { wat flt } \\
\text { mg/L } \\
\text { (70300) }
\end{gathered}
$$

$1.4 \quad 20$

282

55.6

34.7

20.4

2.22

- -

$--\quad \quad-$

$$
--
$$$$
\text { - - }
$$

- -

- -

- -

46

27.3

- -

6.34

$<0.100$

$<1.00$

dried @

Date

wat flt

MAY

$20 \ldots$
$20 .$.

340 
430723088252100 OKAUCHEE LAKE AT OKAUCHEE, WI

LAKE-DEPTH PROFILES, MARCH 6 TO AUGUST 22, 2014

03-06-14

05-20-14

06-16-14

07-24-14

08-22-14

DISSOLVED OXYGEN (D.O.), IN MILLIGRAMS PER LITER
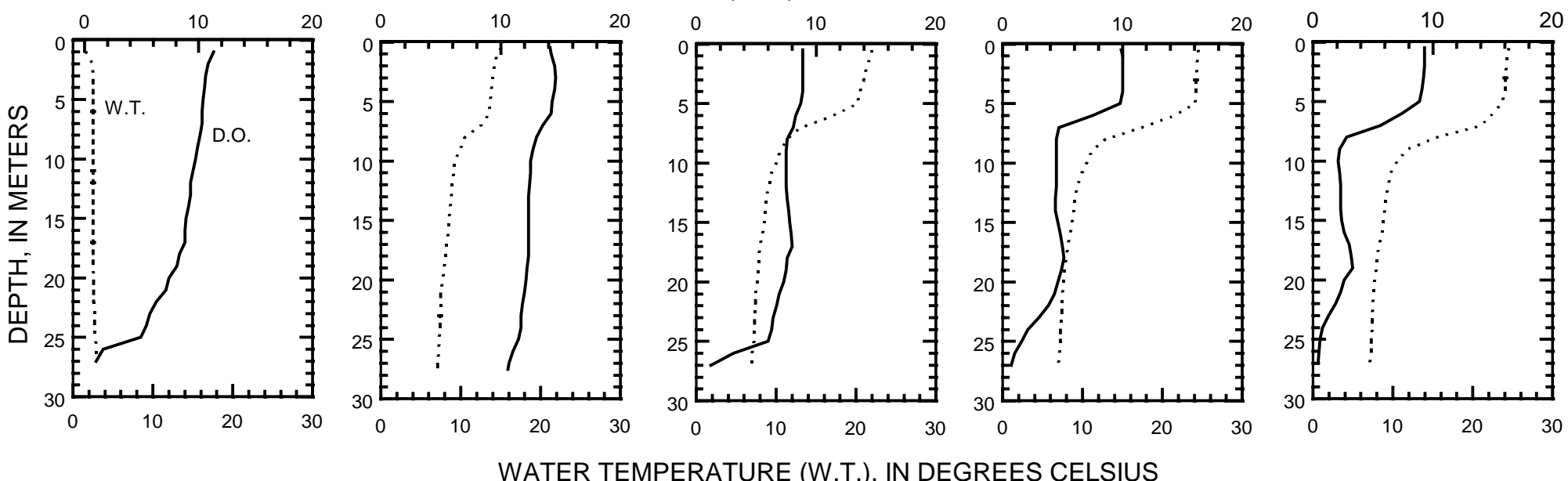

WATER TEMPERATURE (W.T.), IN DEGREES CELSIUS
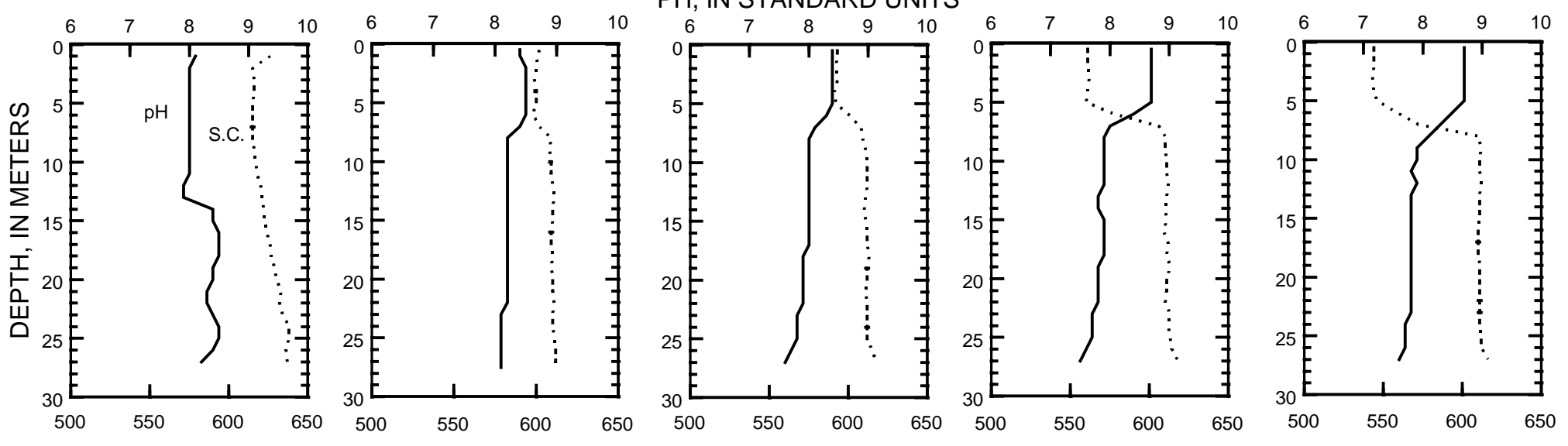

SPECIFIC CONDUCTANCE (S.C.), IN MICROSIEMENS PER CENTIMETER AT 25 DEGREES CELSIUS 

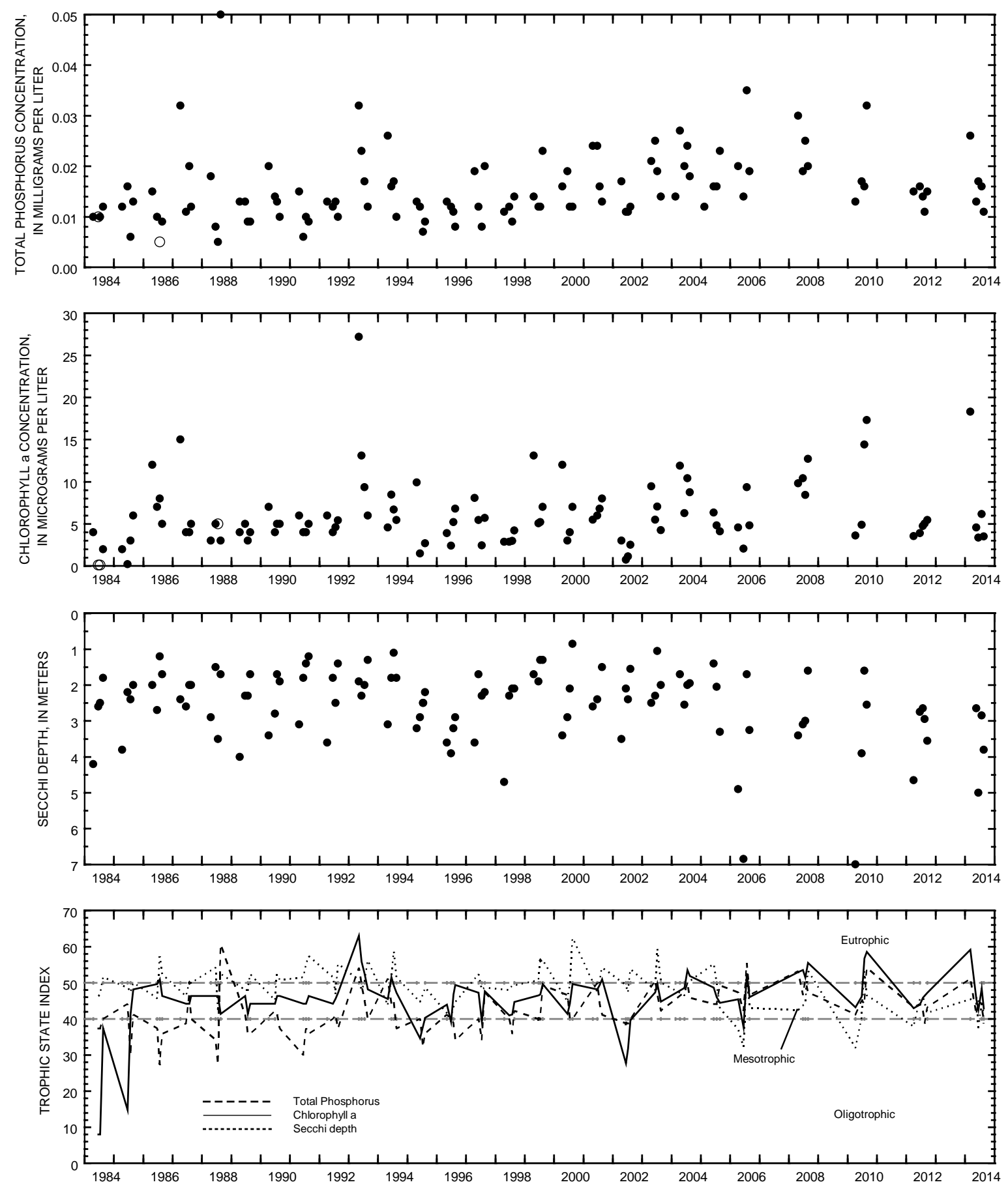

Surface total phosphorus, chlorophyll a concentrations, Secchi depths, and TSI data for Okauchee Lake, near Okauchee, Wisconsin.

(Open circles on the first two plots indicate laboratory detection limit for selected analyses Actual concentrations for these particular analyses are less than the plotted circles.) 


\section{OKAUCHEE LAKE, NO. 1, NEAR OKAUCHEE, WI}

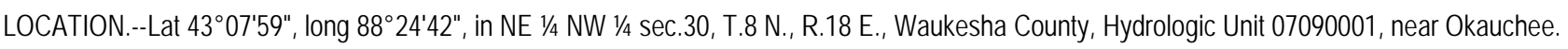

PERIOD OF RECORD.--April 1986 to September 2006, April to August 2008, April to August 2010, April to August 2012, March to August 2014.

REMARKS.--Lake sampled in Crane's Nest Bay, in the northeast part of the lake, at an approximate depth of $2 \mathrm{~m}$. Water-quality analyses done by Wisconsin State Laboratory of Hygiene.

WATER-QUALITY DATA, JUNE 16 TO AUGUST 22, 2014

(Milligrams per liter unless otherwise indicated)

\begin{tabular}{|c|c|c|c|c|c|c|c|c|c|}
\hline Date & $\begin{array}{c}\text { Trans- } \\
\text { parency } \\
\text { Secchi } \\
\text { disc, } \\
\text { meters } \\
(\odot \odot \odot 78)\end{array}$ & $\begin{array}{c}\text { Sam- } \\
\text { pling } \\
\text { depth, } \\
\text { meters } \\
(0 \odot \odot 98)\end{array}$ & $\begin{array}{c}\text { Temper - } \\
\text { ature, } \\
\text { water, } \\
\text { deg C } \\
(\odot \odot \odot 10)\end{array}$ & $\begin{array}{c}\text { Specif- } \\
\text { ic } \\
\text { conduc- } \\
\text { tance, } \\
\text { wat unf } \\
\text { us/cm @ } \\
25 \text { degc } \\
(\odot \odot \odot 95)\end{array}$ & $\begin{array}{c}\text { pH, } \\
\text { water, } \\
\text { unfltrd } \\
\text { field, } \\
\text { std } \\
\text { units } \\
(\odot \odot 4 \odot \odot)\end{array}$ & $\begin{array}{c}\text { Dis- } \\
\text { solved } \\
\text { oxygen, } \\
\text { mg/L } \\
(\odot \odot 30 \odot)\end{array}$ & $\begin{array}{c}\text { Chloro- } \\
\text { phyll a } \\
\text { trichro } \\
\text {-matic } \\
\text { method, } \\
\text { uncorr, } \\
\text { ug/L } \\
(32210)\end{array}$ & $\begin{array}{c}\text { Phos- } \\
\text { phorus, } \\
\text { water, } \\
\text { unfltrd } \\
\text { mg/L } \\
\text { as } P \\
(00665)\end{array}$ & $\begin{array}{c}\text { Ammonia } \\
+ \\
\text { org-N, } \\
\text { water, } \\
\text { unfltrd } \\
\text { mg/L } \\
\text { as N } \\
(0 \odot 625)\end{array}$ \\
\hline
\end{tabular}

JUN 2014

$\begin{array}{rccccccccc}16 \ldots & >1.60 & -- & -- & -- & -- & -- & -- & -- & -- \\ 16 \ldots & -- & 0.50 & 23.5 & 604 & 8.5 & 9.4 & 4.05 & 0.019 & 0.60 \\ \text { JUL } & >1.60 & -- & -- & -- & -- & -- & -- & -- & -- \\ 24 \ldots & -- & 0.50 & 24.9 & 630 & 8.3 & 8.1 & 3.62 & 0.022 & -- \\ 24 \ldots & >1.60 & -- & -- & -- & -- & -- & -- & -- & -- \\ \text { AUG } & -. & 0.50 & 24.7 & 606 & 8.5 & 10.4 & 7.93 & 0.017 & --\end{array}$



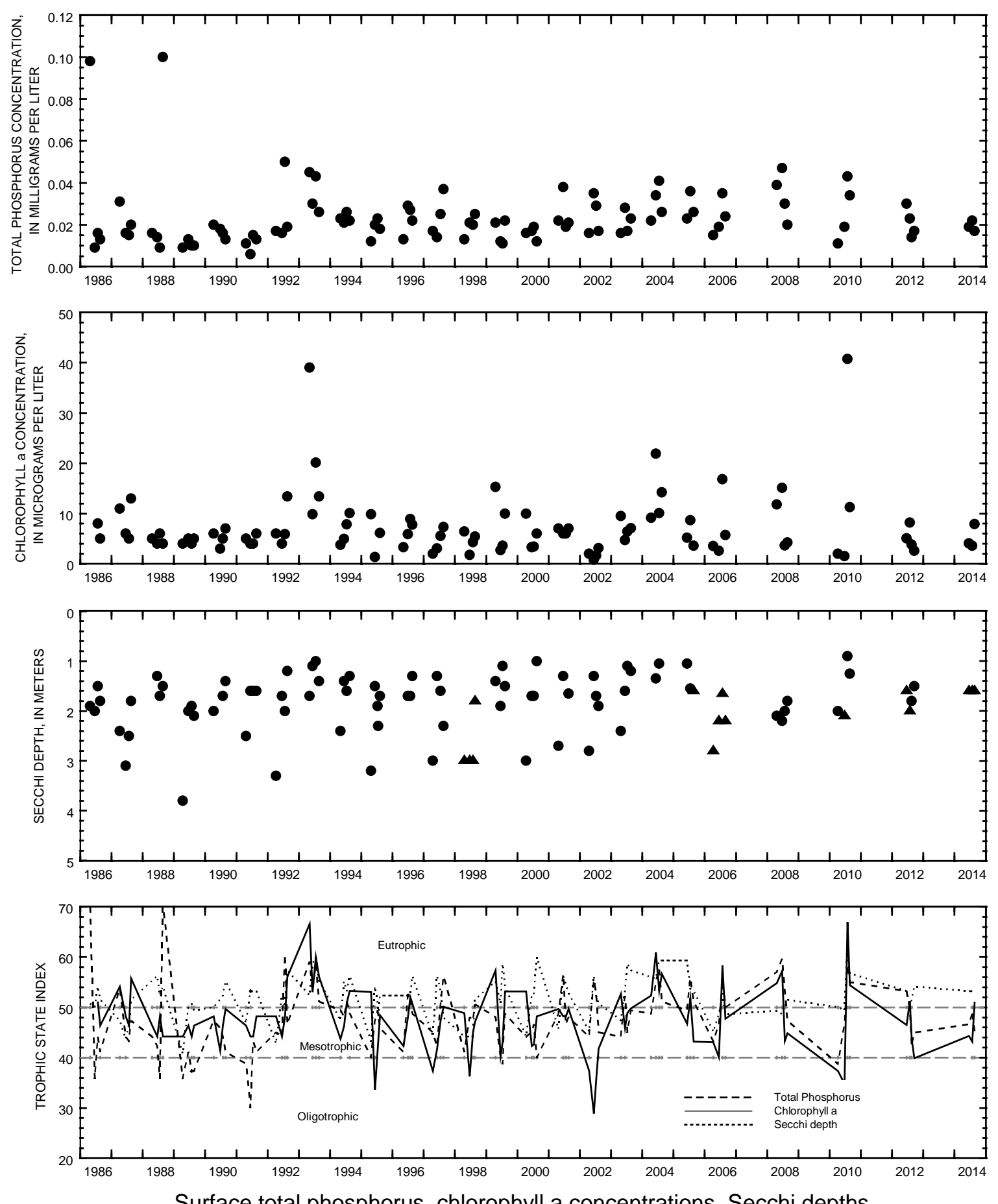

Surface total phosphorus, chlorophyll a concentrations, Secchi depths, and TSI data for Okauchee Lake, No. 1, near Okauchee, Wisconsin.

(Triangles in Secchi plot indicate maximum depth at sampling site. Actual Secchi depth on these days was greater than the plotted triangles.) 


\section{OKAUCHEE LAKE, NO. 2, AT OKAUCHEE, WI}

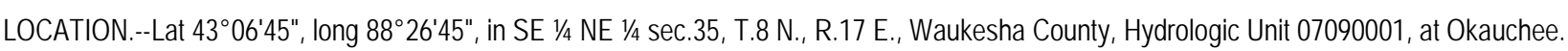

PERIOD OF RECORD.--April 1986 to September 2006, April to August 2008, April to August 2010, April to August 2012, March to August 2014.

REMARKS.--Lake sampled in Lower Okauchee Lake, at an approximate depth of $3 \mathrm{~m}$. Water-quality analyses done by Wisconsin State Laboratory of Hygiene.

WATER-QUALITY DATA, JUNE 16 TO AUGUST 22, 2014

(Milligrams per liter unless otherwise indicated)

\begin{tabular}{|c|c|c|c|c|c|c|c|c|c|}
\hline te & $\begin{array}{c}\text { Trans- } \\
\text { parency } \\
\text { Secchi } \\
\text { disc, } \\
\text { meters } \\
(00078)\end{array}$ & $\begin{array}{c}\text { Sam- } \\
\text { pling } \\
\text { depth, } \\
\text { meters } \\
(00098)\end{array}$ & $\begin{array}{c}\text { Temper- } \\
\text { ature, } \\
\text { water, } \\
\text { deg C } \\
(00010)\end{array}$ & $\begin{array}{c}\text { Specif- } \\
\text { ic } \\
\text { conduc- } \\
\text { tance, } \\
\text { wat unf } \\
\text { uS/cm @ } \\
25 \text { degc } \\
\text { (00095) }\end{array}$ & $\begin{array}{l}\mathrm{pH}, \\
\text { water, } \\
\text { unfltrd } \\
\text { field, } \\
\text { std } \\
\text { units } \\
(00400)\end{array}$ & $\begin{array}{c}\text { Dis- } \\
\text { solved } \\
\text { oxygen, } \\
\text { mg/L } \\
(00300)\end{array}$ & $\begin{array}{l}\text { Chloro- } \\
\text { phyll a } \\
\text { trichro } \\
\text {-matic } \\
\text { method, } \\
\text { uncorr, } \\
\text { ug/L } \\
(32210)\end{array}$ & $\begin{array}{l}\text { Phos- } \\
\text { phorus, } \\
\text { water, } \\
\text { unfltrd } \\
\mathrm{mg} / \mathrm{L} \\
\text { as } \mathrm{P} \\
(00665)\end{array}$ & $\begin{array}{c}\text { Ammonia } \\
+ \\
\text { org-N, } \\
\text { water, } \\
\text { unfltrd } \\
\mathrm{mg} / \mathrm{L} \\
\text { as N } \\
(00625)\end{array}$ \\
\hline
\end{tabular}

JUN 2014

$16 \ldots$

$>2.80$

$\odot .5$

23.8

571

8.4

9.1

2.39

๑.--

0.64

JUL

$>2.80$

$24 \ldots$

AUG 24.

$22 \ldots$

$22 \ldots$

$-$

0.

25.

--
525

- - 10

525

$\begin{array}{ll}8.6 & 10.3 \\ 8.6 & 10.8\end{array}$

$3 . \odot \odot$

- -

$0.50 \quad 25.2 \quad 507$

$\begin{array}{rr}-- & -- \\ 8.8 & 9.2\end{array}$

-

2.5

24.9

507

8.8

9.3

2.

0.016

0.016

3. $48 \quad 0.014$

- $\quad 0.017$ 

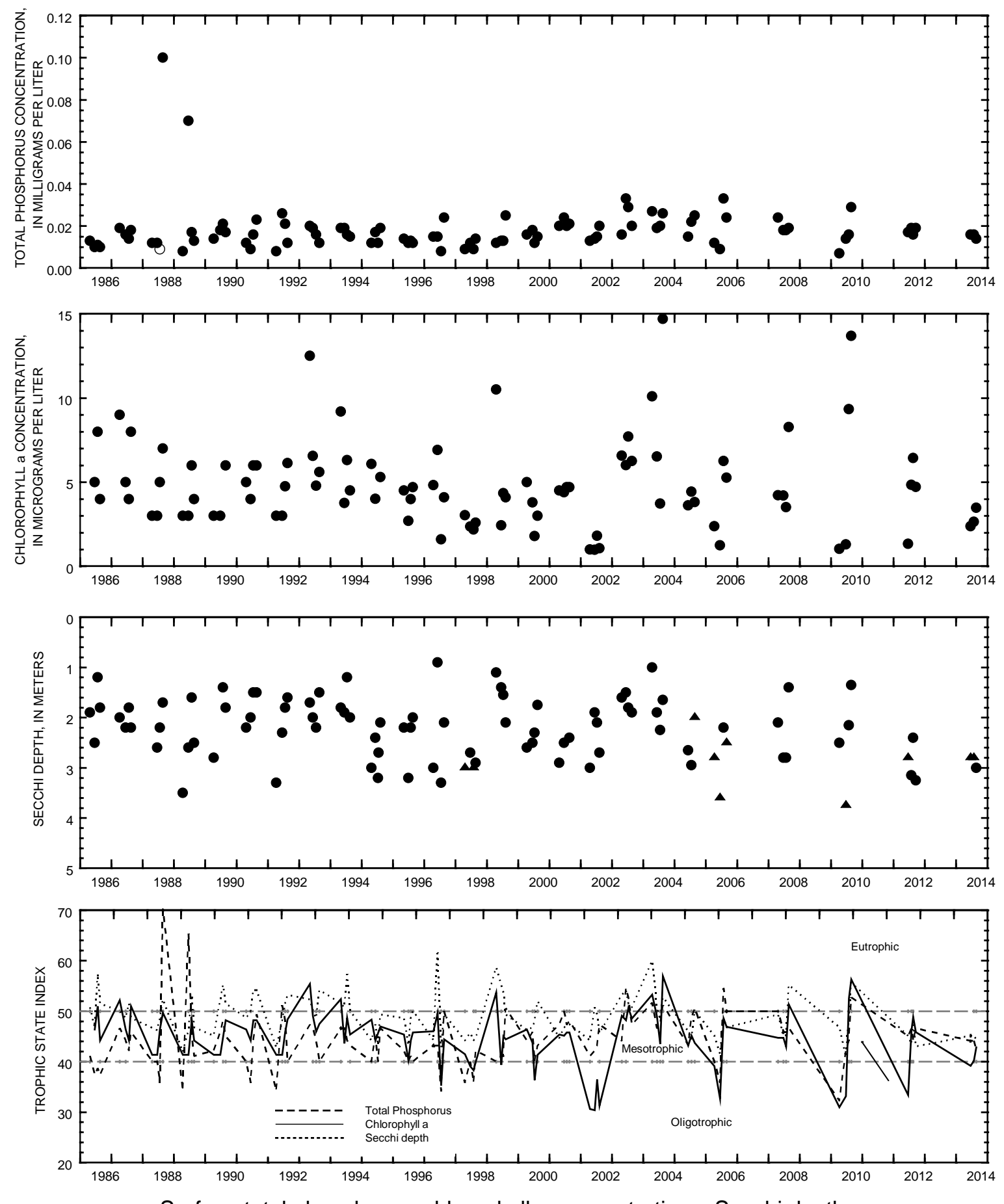

Surface total phosphorus, chlorophyll a concentrations, Secchi depths, and TSI data for Okauchee Lake, No. 2, near Okauchee, Wisconsin.

(Open circles on the first two plots indicate laboratory detection limit for selected analyses. Actual concentrations for these particular analyses are less than the plotted circles.)

(Triangles in Secchi plot indicate maximum depth at sampling site.

Actual Secchi depth on these days was greater than the plotted triangles.) 


\section{OKAUCHEE LAKE, NO. 3, AT OKAUCHEE, WI}

LOCATION.--Lat $43^{\circ} 06^{\prime} 42^{\prime \prime}$ l long $88^{\circ} 25^{\prime} 24^{\prime \prime}$, in NE 1/4 SE 1/4 Sec.36, T.8 N., R.17 E., Waukesha County, Hydrologic Unit 07090001, at Okauchee.

PERIOD OF RECORD.--April 1986 to September 2006, April to August 2008, April to August 2010, April to August 2012, March to August 2014.

REMARKS.--Lake sampled in Ice House Bay, in the southern part of the lake, at an approximate depth of $5 \mathrm{~m}$. Water-quality analyses done by Wisconsin State Laboratory of Hygiene.

WATER-QUALITY DATA, JUNE 16 TO AUGUST 22, 2014

(Milligrams per liter unless otherwise indicated)

\begin{tabular}{|c|c|c|c|c|c|c|c|c|c|}
\hline ate & $\begin{array}{c}\text { Trans- } \\
\text { parency } \\
\text { Secchi } \\
\text { disc, } \\
\text { meters } \\
(\odot \odot \odot 78)\end{array}$ & $\begin{array}{c}\text { Sam- } \\
\text { pling } \\
\text { depth, } \\
\text { meters } \\
(00098)\end{array}$ & $\begin{array}{c}\text { Temper - } \\
\text { ature, } \\
\text { water, } \\
\text { deg C } \\
(00010)\end{array}$ & $\begin{array}{c}\text { Specif- } \\
\text { ic } \\
\text { conduc- } \\
\text { tance, } \\
\text { wat unf } \\
\text { uS/cm @ } \\
25 \mathrm{degC} \\
(00095)\end{array}$ & $\begin{array}{c}\text { pH, } \\
\text { water, } \\
\text { unfltrd } \\
\text { field, } \\
\text { std } \\
\text { units } \\
(00400)\end{array}$ & $\begin{array}{c}\text { Dis- } \\
\text { solved } \\
\text { oxygen, } \\
\text { mg/L } \\
(00300)\end{array}$ & $\begin{array}{c}\text { Chloro- } \\
\text { phyll a } \\
\text { trichro } \\
\text {-matic } \\
\text { method, } \\
\text { uncorr, } \\
\text { ug/L } \\
(32210)\end{array}$ & $\begin{array}{c}\text { Phos- } \\
\text { phorus, } \\
\text { water, } \\
\text { unfltrd } \\
\text { mg/L } \\
\text { as P } \\
(00665)\end{array}$ & $\begin{array}{c}\text { Ammonia } \\
+ \\
\text { org-N, } \\
\text { water, } \\
\text { unfltrd } \\
\text { mg/L } \\
\text { as N } \\
(00625)\end{array}$ \\
\hline
\end{tabular}

JUN 2014

$16 \ldots$

$>5.00$

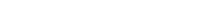

(1)

(0665)

(00625)

JUL .

$24 \ldots$

$24 \ldots$

AUG $24 \ldots$

$22 \ldots$

$22 \ldots$

3.85

$\begin{array}{lr}--\overline{5} & -\overline{7} \\ 0.7 & \end{array}$

573

8.4

9.4

2.15

0.017

0.77

3.85
--
--

0.

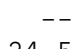

- -

$8 .-$

$--$

$4.5 \quad 23.9$

539

4.

$0 .-017 \quad-$

4.35

$\begin{array}{cr}-- & -- \\ 0.50 & 24.8\end{array}$

--
516

8.6

0.016

$-$

- - - - -

$$
\text { -- }
$$

4.5

23.6

--
8.8
8.6

9.5

$3.45 \quad 0.015$

0.014 

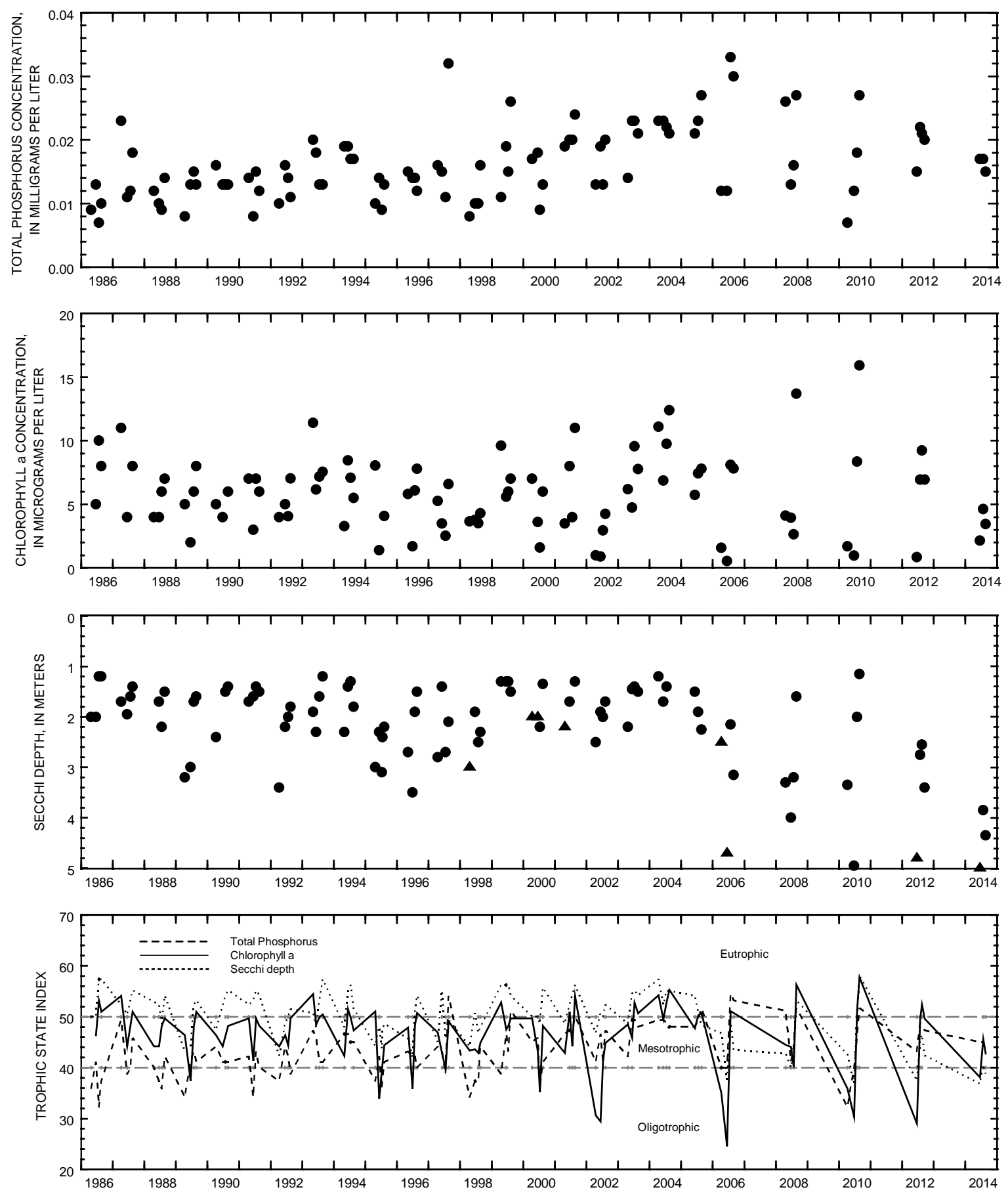

Surface total phosphorus, chlorophyll a concentrations, Secchi depths, and TSI data for Okauchee Lake, No. 3, near Okauchee, Wisconsin. 


\section{OKAUCHEE LAKE, NO. 4, AT OKAUCHEE, WI}

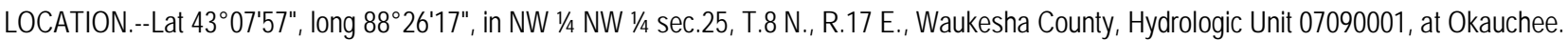

PERIOD OF RECORD.--June 1986 to September 2006, April to August 2008, April to August 2010, April to August 2012, March to August 2014.

REMARKS.--Lake sampled near McDowell (Crazyman's) Island, in the northwest bay of the lake, at an approximate depth of

$2 \mathrm{~m}$. Water-quality analyses done by Wisconsin State Laboratory of Hygiene.

WATER-QUALITY DATA, JUNE 16 TO AUGUST 22, 2014

(Milligrams per liter unless otherwise indicated)

\begin{tabular}{|c|c|c|c|c|c|c|c|c|c|}
\hline Date & $\begin{array}{c}\text { Trans- } \\
\text { parency } \\
\text { Secchi } \\
\text { disc, } \\
\text { meters } \\
(0 \odot \odot 78)\end{array}$ & $\begin{array}{c}\text { Sam- } \\
\text { pling } \\
\text { depth, } \\
\text { meters } \\
(\odot \odot \odot 98)\end{array}$ & $\begin{array}{c}\text { Temper - } \\
\text { ature, } \\
\text { water, } \\
\text { deg C } \\
(00010)\end{array}$ & $\begin{array}{c}\text { Specif - } \\
\text { ic } \\
\text { conduc- } \\
\text { tance, } \\
\text { wat unf } \\
\text { uS } / \mathrm{cm} @ \\
25 \mathrm{degC} \\
(\odot \odot \odot 95)\end{array}$ & $\begin{array}{c}\text { pH, } \\
\text { water, } \\
\text { unfltrd } \\
\text { field, } \\
\text { std } \\
\text { units } \\
(\odot \odot 4 \odot \odot)\end{array}$ & $\begin{array}{c}\text { Dis- } \\
\text { solved } \\
\text { oxygen, } \\
\text { mg/L } \\
(00300)\end{array}$ & $\begin{array}{c}\text { Chloro- } \\
\text { phyll a } \\
\text { trichro } \\
\text { - matic } \\
\text { method, } \\
\text { uncorr, } \\
\text { ug/L } \\
(32210)\end{array}$ & $\begin{array}{c}\text { Phos- } \\
\text { phorus, } \\
\text { water, } \\
\text { unfltrd } \\
\text { mg/L } \\
\text { as P } \\
(00665)\end{array}$ & $\begin{array}{c}\text { Ammonia } \\
+ \\
\text { org-N, } \\
\text { water, } \\
\text { unfltrd } \\
\text { mg/L } \\
\text { as N } \\
(0 \odot 625)\end{array}$ \\
\hline
\end{tabular}

JUN 2014

$\begin{array}{rccccccccc}16 \ldots & >1.50 & -- & -- & -- & -- & -- & -- & -- & -- \\ 16 \ldots & -- & 0.50 & 22.4 & 560 & 8.7 & 14.1 & 1.52 & 0.015 & 0.77 \\ \text { JUL } & >1.50 & -- & -- & -- & -- & -- & -- & -- & -- \\ 24 \ldots & -- & 0.50 & 24.6 & 522 & 8.8 & 10.3 & 2.20 & 0.016 & -- \\ 24 \ldots & >1.50 & -- & -- & -- & -- & -- & -- & -- & -- \\ \text { AUG } & -- & 0.50 & 25.2 & 522 & 8.7 & 8.5 & 3.39 & 0.014 & --\end{array}$



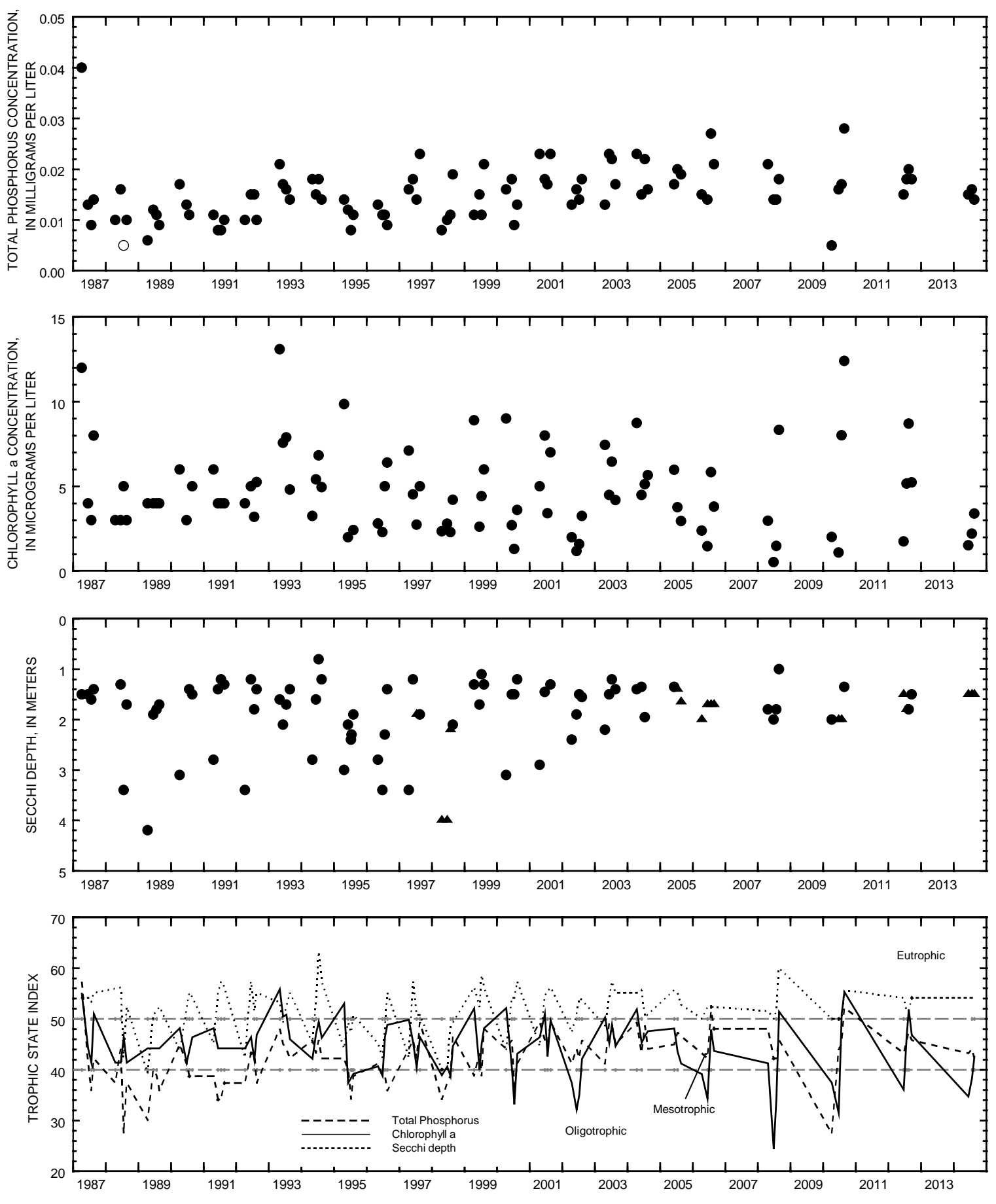

Surface total phosphorus, chlorophyll a concentrations, Secchi depths, and TSI data for Okauchee Lake, No. 4, near Okauchee, Wisconsin.

(Open circles on the first two plots indicate laboratory detection limit for selected analyses. Actual concentrations for these particular analyses are less than the plotted circles.)

(Triangles in Secchi plot indicate maximum depth at sampling site.

Actual Secchi depth on these days was greater than the plotted triangles.) 
LOCATION.--Lat $42^{\circ} 32^{\prime} 46^{\prime \prime}$, long $88^{\circ} 17^{\prime} 58^{\prime \prime}$, in NW 1/4 SE 1/4 Sec.13, T.1 N., R.18 E., Walworth County, Hydrologic Unit 07120006, at Powers Lake.

SURFACE AREA. $-0.72 \mathrm{mi}^{2}$.

DRAINAGE AREA.--3.42 $\mathrm{mi}^{2}$

PERIOD OF RECORD.--March 1986 to August 1996, and April 1998 to current year.

REMARKS.--Lake sampled near center at the deep hole. Water-quality analyses done by Wisconsin State Laboratory of Hygiene.

WATER-QUALITY DATA, MARCH 4 TO AUGUST 19, 2014

(Milligrams per liter unless otherwise indicated)

\begin{tabular}{|c|c|c|c|c|c|c|c|c|c|c|c|c|c|}
\hline Date & $\begin{array}{c}\text { Trans- } \\
\text { parency } \\
\text { Secchi } \\
\text { disc, } \\
\text { meters } \\
(00078)\end{array}$ & $\begin{array}{c}\text { Sam- } \\
\text { pling } \\
\text { depth, } \\
\text { meters } \\
(\odot \odot 098)\end{array}$ & $\begin{array}{c}\text { Temper - } \\
\text { ature, } \\
\text { water, } \\
\text { deg C } \\
(00010)\end{array}$ & $\begin{array}{c}\text { Specif- } \\
\text { ic } \\
\text { conduc- } \\
\text { tance, } \\
\text { wat unf } \\
\text { us/cm @ } \\
25 \mathrm{degC} \\
(0 \odot \odot 95)\end{array}$ & $\begin{array}{c}\text { pH, } \\
\text { water, } \\
\text { unfltrd } \\
\text { field, } \\
\text { std } \\
\text { units } \\
(0040 \odot)\end{array}$ & $\begin{array}{c}\text { Dis- } \\
\text { solved } \\
\text { oxygen, } \\
\text { mg/L } \\
(00300)\end{array}$ & $\begin{array}{c}\text { Chloro- } \\
\text { phyll a } \\
\text { trichro } \\
\text {-matic } \\
\text { method, } \\
\text { uncorr, } \\
\text { ug/L } \\
(32210)\end{array}$ & $\begin{array}{c}\text { Phos- } \\
\text { phorus, } \\
\text { water, } \\
\text { unfltrd } \\
\text { mg/L } \\
\text { as P } \\
(00665)\end{array}$ & $\begin{array}{l}\text { Ortho- } \\
\text { phos- } \\
\text { phate, } \\
\text { water, } \\
\text { fltrd, } \\
\text { mg/L } \\
\text { as P } \\
(00671)\end{array}$ & $\begin{array}{c}\text { Total } \\
\text { nitro- } \\
\text { gen, } \\
\text { water, } \\
\text { unfltrd } \\
\mathrm{mg} / \mathrm{L} \\
(00600)\end{array}$ & $\begin{array}{c}\text { Ammonia } \\
\text { water, } \\
\text { fltrd, } \\
\text { mg/L } \\
\text { as N } \\
(00608)\end{array}$ & $\begin{array}{c}\text { Ammonia } \\
+ \\
\text { org-N, } \\
\text { water, } \\
\text { fltrd, } \\
\text { mg/L } \\
\text { as N } \\
(00623)\end{array}$ & $\begin{array}{c}\text { Ammonia } \\
+ \\
\text { org-N, } \\
\text { water, } \\
\text { unfltrd } \\
\text { mg/L } \\
\text { as N } \\
(00625)\end{array}$ \\
\hline \multicolumn{14}{|l|}{ MAR 2014} \\
\hline$\odot 4 \ldots$ & -- & 1.0 & 2.7 & 579 & 8.1 & 12.4 & 4.18 & 0.013 & - - & -- & - - & - - & - - \\
\hline \multicolumn{14}{|l|}{ MAY } \\
\hline $20 \ldots$ & 5.50 & -- & -- & -- & -- & - - & -- & -- & - - & -- & -- & - - & -- \\
\hline $20 \ldots$ & -- & 0.50 & 15.2 & 519 & 8.3 & 11.7 & 1.32 & 0.011 & $<0.002$ & $<0.66$ & 0.026 & - - & 0.64 \\
\hline \multicolumn{14}{|l|}{ JUN } \\
\hline $30 \ldots$ & 3.55 & - - & -- & -- & -- & -- & -- & - & -- & -- & - & -- & -- \\
\hline $30 \ldots$ & -- & 0.50 & 25.4 & 498 & 8.5 & 8.4 & 4.59 & $\odot .018$ & - - & - - & - - & - - & - - \\
\hline $30 \ldots$ & -- & $9 . \odot$ & 14.7 & 535 & 7.5 & 0.2 & - - & $\odot .039$ & -- & -- & - & -- & -- \\
\hline \multicolumn{14}{|l|}{ JUL } \\
\hline $29 \ldots$ & 3.30 & - - & - - & - - & - - & - - & - - & - - & - - & - - & - - & - - & - - \\
\hline $29 \ldots$ & -- & 0.50 & 24.0 & 492 & 8.6 & 8.4 & 3.88 & 0.017 & $<0.002$ & $<0.57$ & $<0.015$ & 0.71 & 0.56 \\
\hline $19 \ldots$ & 2.95 & - - & -- & -- & -- & -- & -- & -- & -- & -- & -- & -- & -- \\
\hline $19 \ldots$ & - - & 0.50 & 24.5 & 510 & 8.5 & 8.8 & 4.52 & 0.016 & -- & - - & - - & - - & -- \\
\hline $19 \ldots$ & - - & 9.5 & 15.7 & 544 & 7.5 & 0.5 & -- & 0.049 & - - & - - & -- & -- & -- \\
\hline
\end{tabular}




\section{POWERS LAKE AT POWERS LAKE, WI}

WATER-QUALITY DATA, MARCH 4 TO AUGUST 19, 2014

(Milligrams per liter unless otherwise indicated)

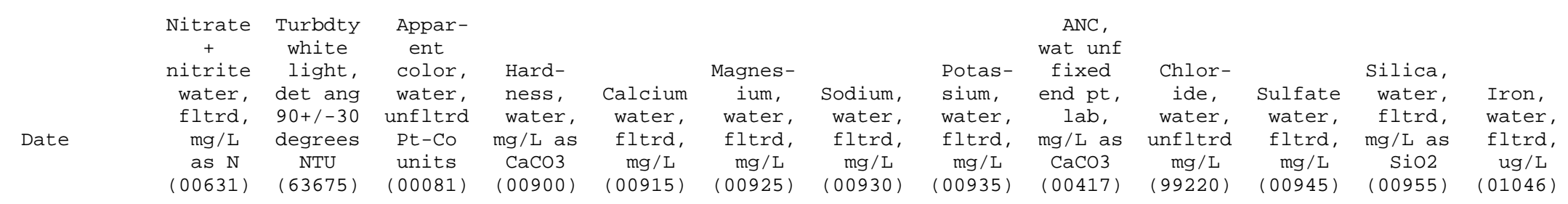

MAR 2014

$04 \ldots$

$04 \ldots$

MAY

$20 \ldots$
$20 \ldots$

JUN

$30 \ldots$

$30 \ldots$

$30 \ldots$

JUL

$29 \ldots$

$29 \ldots$

AUG

$19 \ldots$

$19 \ldots$

$19 \ldots$

$\begin{array}{ccc}-- & -- & - \\ -- & -- & -- \\ -- & -- & -- \\ <0.019 & 0.7 & 15 \\ -- & -- & -- \\ -- & -- & - \\ -- & -- & -- \\ -- & -- & -- \\ <0.019 & -- & - \\ -- & -- & - \\ -- & -- & -- \\ -- & -- & -- \\ -- & -- & --\end{array}$

$\begin{array}{rrr}-- & -- & - \\ -- & -- & - \\ -- & -- & - \\ 218 & 35.8 & 31.3 \\ -- & -- & -- \\ -- & -- & - \\ -- & -- & -- \\ -- & -- & -- \\ -- & -- & - \\ -- & -- & - \\ -- & -- & - \\ -- & -- & -- \\ -- & -- & --\end{array}$

\begin{tabular}{|c|c|c|}
\hline & $\begin{array}{l}\text { Manga- } \\
\text { nese, } \\
\text { water, }\end{array}$ & $\begin{array}{c}\text { Dis- } \\
\text { solved } \\
\text { solids } \\
\text { dried @ } \\
\text { 180degC }\end{array}$ \\
\hline Date & $\begin{array}{c}\text { fltrd, } \\
\text { ug/L } \\
(01056)\end{array}$ & $\begin{array}{c}\text { wat flt } \\
\mathrm{mg} / \mathrm{L} \\
(7030 \odot)\end{array}$ \\
\hline
\end{tabular}

MAY

$\begin{array}{lrr}20 \ldots & -- & -- \\ 20 \ldots & <1.00 & 286\end{array}$ 
423246088175800 POWERS LAKE AT POWERS LAKE, WI

LAKE-DEPTH PROFILES, MARCH 4 TO AUGUST 19, 2014

03-04-14
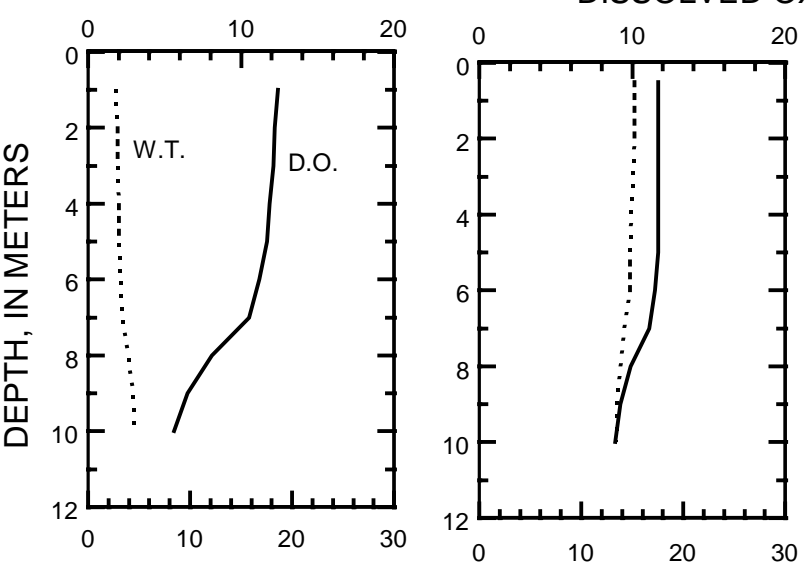

06-30-14

07-29-14

08-19-14

DISSOLVED OXYGEN (D.O.), IN MILLIGRAMS PER LITER

WATER TEMPERATURE (W.T.), IN DEGREES CELSIUS
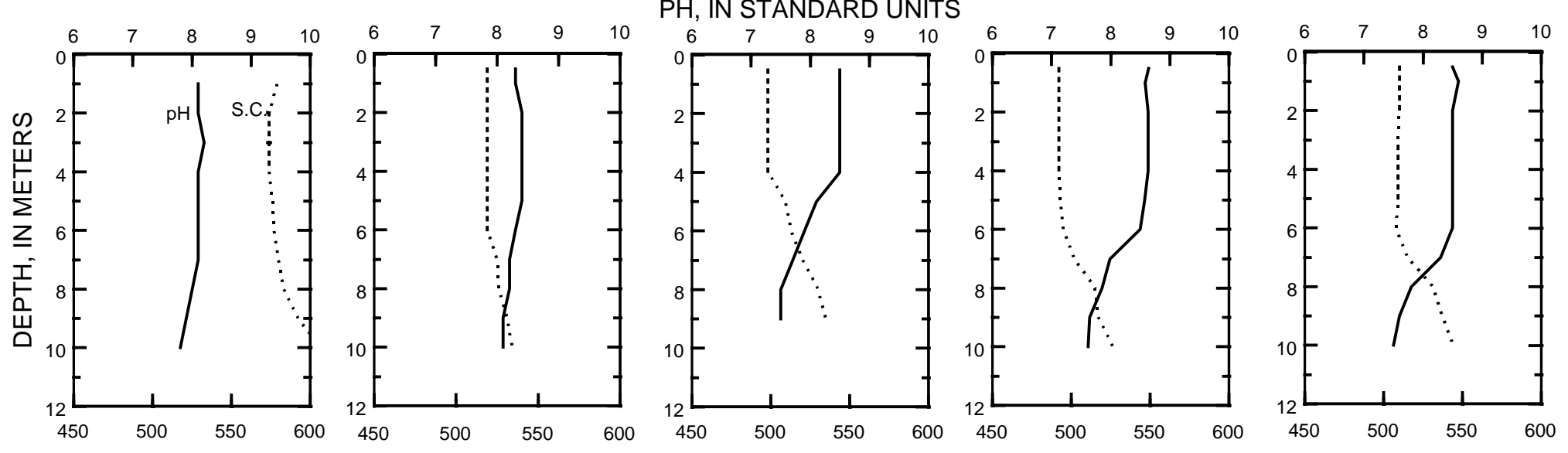

SPECIFIC CONDUCTANCE (S.C.), IN MICROSIEMENS PER CENTIMETER AT 25 DEGREES CELSIUS 

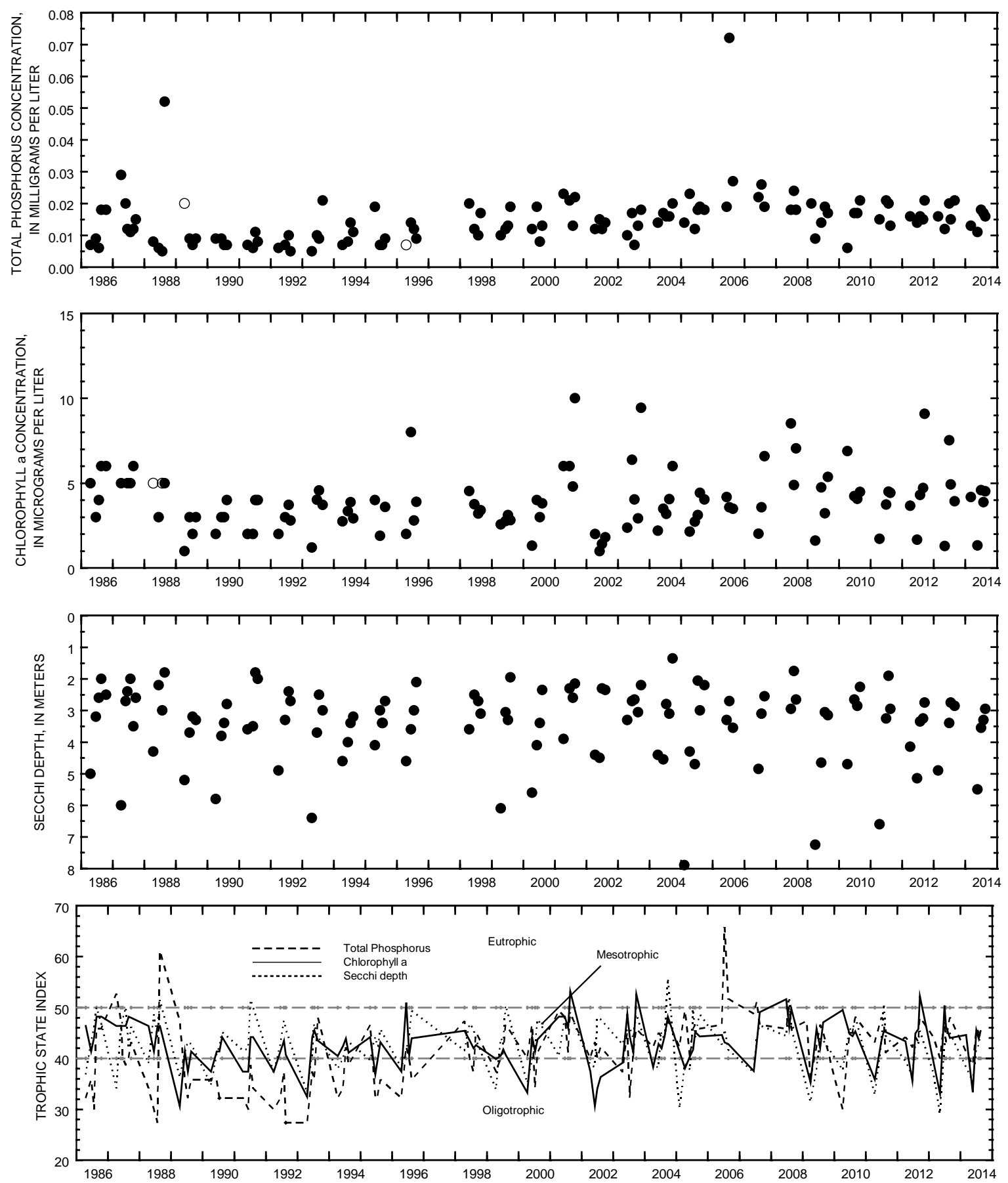

Surface total phosphorus, chlorophyll a concentrations, Secchi depths, and TSI data for Powers Lake, at Powers Lake, Wisconsin.

(Open circles on the first two plots indicate laboratory detection limit for selected analyses. Actual concentrations for these particular analyses are less than the plotted circles.) 


\section{SILVER LAKE NEAR CUMBERLAND, WI}

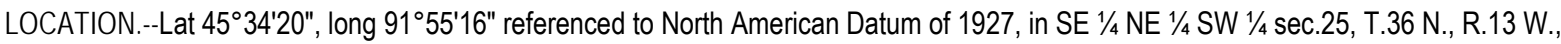
Barron County, WI, Hydrologic Unit 07050007.

DRAINAGE AREA.--1,800 acres; $2.81 \mathrm{mi}^{2}$

PERIOD OF RECORD.--October 2011 to current year. Archived data October 2004 to September 2011.

GAGE.--Non-recording gage. Staff gage mounted on wood board and 1.5" steel pipe. The staff is installed/removed seasonally to coincide with measuring stage during the open water period.

COOPERATION.--Wisconsin Department of Natural Resources.

REMARKS.--Water surface elevation collected by land owner (observer).

EXTREMES FOR PERIOD OF RECORD.--Maximum daily gage height, $80.66 \mathrm{ft}$, June 30, 2014; Minimum daily gage height, $78.10 \mathrm{ft}$, Nov. 7, 2013.

EXTREMES FOR CURRENT YEAR.--Maximum daily gage height, $80.66 \mathrm{ft}$, June 30; Minimum daily gage height, $78.10 \mathrm{ft}$, Nov. 7.

GAGE HEIGHT, FEET

WATER YEAR OCTOBER 2013 TO SEPTEMBER 2014

\begin{tabular}{|c|c|c|c|c|c|c|c|c|c|c|c|c|}
\hline DAY & OCT & NOV & DEC & JAN & FEB & MAR & APR & MAY & JUN & JUL & AUG & SEP \\
\hline 1 & --- & --- & -- & --- & --- & --- & --- & --- & --- & -- & --- & --- \\
\hline 2 & -- & -- & -- & --- & --- & --- & --- & --- & 80.40 & --- & --- & --- \\
\hline 3 & --- & --- & -- & --- & --- & --- & --- & --- & --- & --- & --- & --- \\
\hline 4 & --- & --- & --- & --- & --- & --- & --- & --- & --- & --- & --- & --- \\
\hline 5 & -- & --- & --- & --- & --- & --- & --- & --- & --- & -- & -- & --- \\
\hline 6 & -- & -- & -- & --- & -- & -- & -- & -- & -- & -- & -- & -- \\
\hline 7 & -- & 78.10 & -- & --- & --- & --- & --- & --- & --- & 80.55 & --- & --- \\
\hline 8 & -- & -- & --- & -- & -- & --- & -- & -- & -- & -- & -- & -- \\
\hline 9 & -- & -- & -- & -- & -- & -- & -- & -- & -- & -- & -- & -- \\
\hline 10 & -- & -- & -- & -- & -- & --- & --- & --- & --- & -- & -- & --- \\
\hline 11 & --- & --- & --- & -- & -- & -- & --- & -- & -- & -- & -- & -- \\
\hline 12 & -- & -- & --- & -- & --- & --- & --- & --- & -- & 80.52 & 80.08 & --- \\
\hline 13 & -- & -- & -- & -- & -- & -- & -- & -- & -- & -- & -- & --- \\
\hline 14 & -- & -- & -- & -- & --- & --- & --- & --- & --- & -- & -- & 80.32 \\
\hline 15 & --- & --- & --- & --- & --- & -- & -- & -- & -- & --- & -- & -- \\
\hline 16 & 78.30 & -- & --- & --- & -- & -- & -- & -- & -- & -- & -- & -- \\
\hline 17 & --- & -- & --- & -- & --- & -- & -- & -- & -- & 80.43 & --- & -- \\
\hline 18 & -- & -- & --- & --- & -- & -- & -- & -- & -- & -- & 79.98 & -- \\
\hline 19 & --- & --- & --- & --- & --- & --- & --- & --- & --- & --- & --- & --- \\
\hline 20 & -- & -- & -- & -- & -- & --- & --- & --- & --- & --- & --- & -- \\
\hline 21 & --- & --- & --- & --- & --- & -- & -- & --- & --- & 80.34 & --- & --- \\
\hline 22 & 78.26 & --- & --- & --- & -- & --- & --- & --- & --- & -- & 80.06 & --- \\
\hline 23 & --- & --- & --- & --- & -- & --- & --- & --- & --- & -- & --- & --- \\
\hline 24 & --- & -- & -- & --- & --- & --- & --- & --- & --- & -- & --- & --- \\
\hline 25 & -- & -- & --- & --- & --- & --- & --- & --- & --- & --- & --- & --- \\
\hline 26 & --- & --- & --- & --- & -- & --- & --- & --- & --- & -- & -- & 80.20 \\
\hline 29 & -- & -- & -- & --- & --- & -- & --- & 80.26 & --- & --- & --- & --- \\
\hline 30 & --- & --- & --- & --- & --- & --- & --- & --- & 80.66 & 80.22 & --- & --- \\
\hline 31 & --- & --- & --- & --- & --- & --- & --- & --- & -- & --- & --- & --- \\
\hline
\end{tabular}




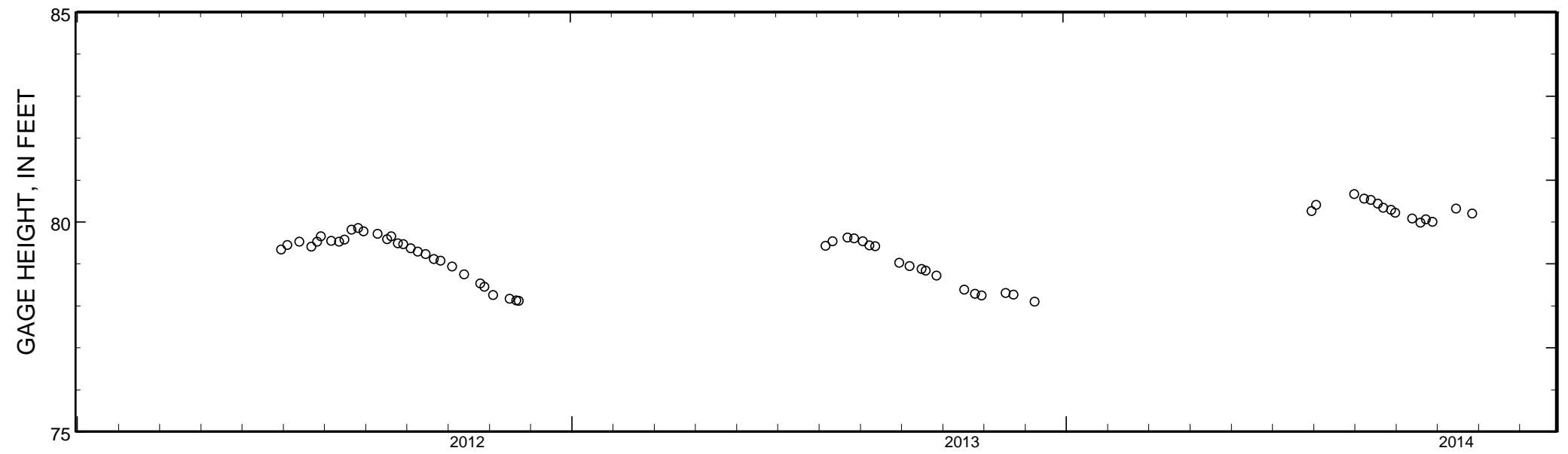

Stage hydrograph for Silver Lake near Cumberland, 2011-2014. 


\section{SILVER LAKE AT PORTAGE, WI}

LOCATION.--Lat 433''10", long 89²8'22.9" referenced to North American Datum of 1983, Columbia County, WI, Hydrologic Unit 04030201 , previously published at Lat $43^{\circ} 33^{\prime} 10^{\prime \prime}$, long $89^{\circ} 07^{\prime} 58^{\prime \prime}$ referenced to North American Datum of 1927

DRAINAGE AREA.--1.00 mi².

PERIOD OF RECORD.--May 2010 to current year. Work records from August 1936 to February 2010 in USGS database.

GAGE.--A staff gage is periodically read by an observer, and reported in local datum.

COOPERATION.--Wisconsin Department of Natural Resources.

EXTREMES FOR PERIOD OF RECORD.--Maximum observed gage height, $8.88 \mathrm{ft}$ July 21, 2010; miminum observed gage height, $6.20 \mathrm{ft}$, Oct. 12, 2012.

EXTREMES FOR CURRENT YEAR.--Maximum observed gage height, $8.49 \mathrm{ft}$ May 13; miminum observed gage height, $7.60 \mathrm{ft}$, Oct. 3, 2013.

GAGE HEIGHT, FEET

WATER YEAR OCTOBER 2013 TO SEPTEMBER 2014

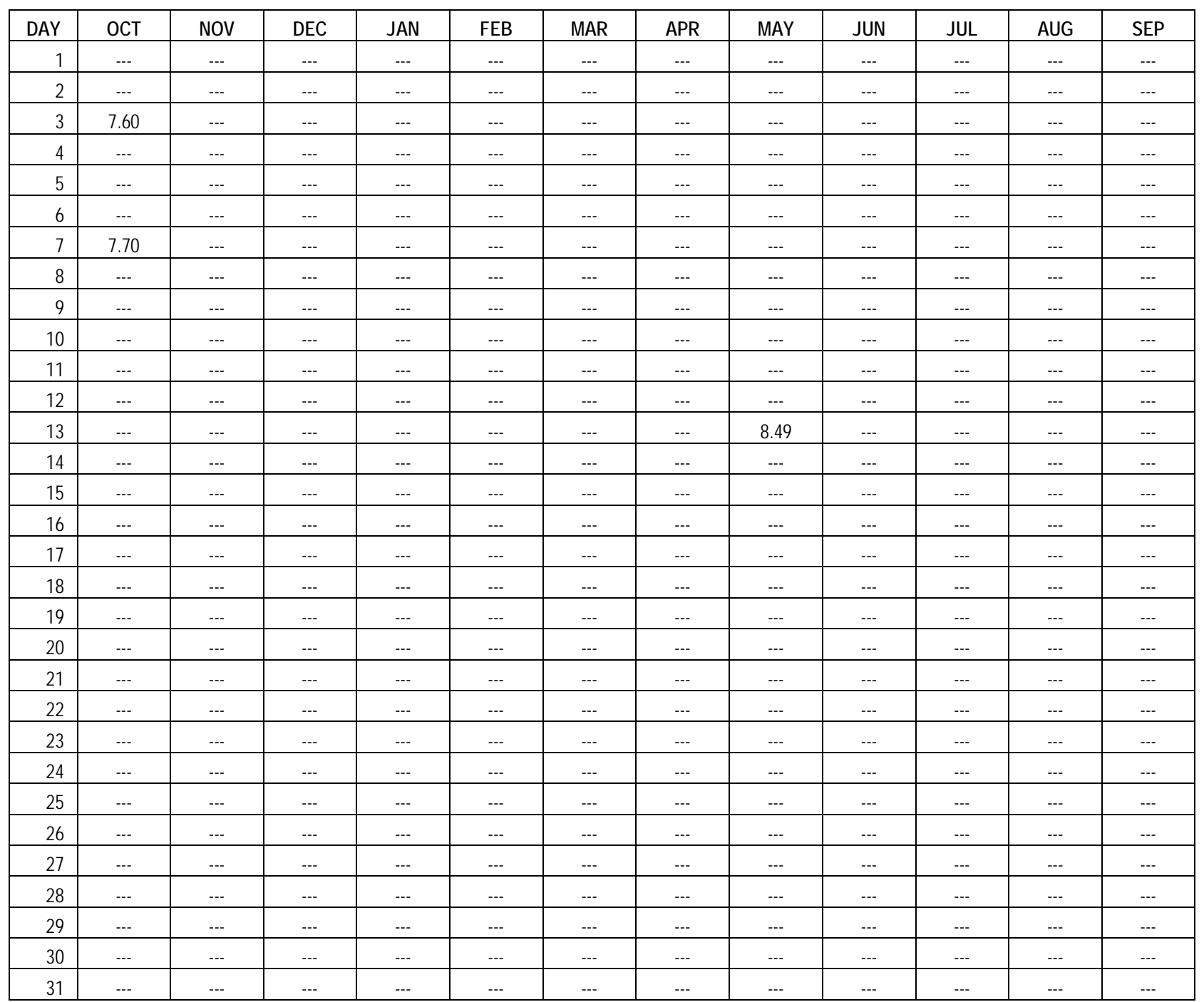




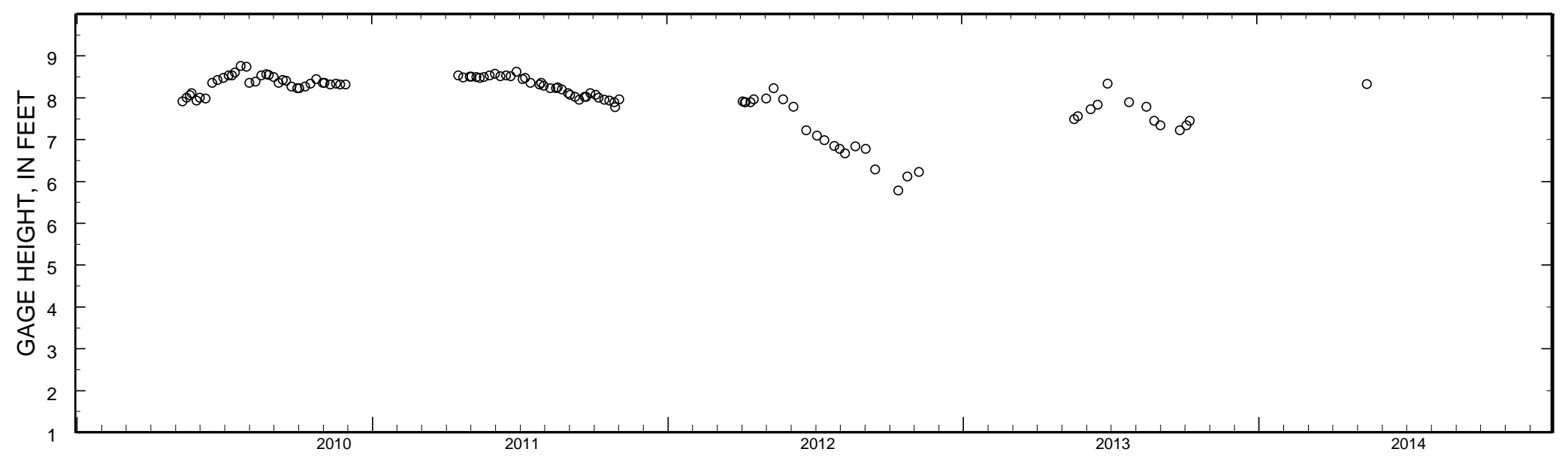

Stage hydrograph for Silver Lake at Portage, 2010-2014. 


\section{LAKE WAUBESA AT MCFARLAND, WI}

LOCATION.--Lat 4300'32", long 89¹8'19" referenced to North American Datum of 1927, in SW 1/4 SW 1/4 sec.3, T.6 N., R.10 E., Dane County, WI, Hydrologic Unit 07090001, on left bank just upstream from bridge on U.S. Highway 51, downstream of dam at outlet of Lake Waubesa and 1.0 mi southwest of McFarland.

SURFACE AREA.--3.25 mi².

DRAINAGE AREA.--327 $\mathrm{mi}^{2}$ of which $36.6 \mathrm{mi}^{2}$ probably is noncontributing.

PERIOD OF RECORD.--October 2003 to current year.

REVISED RECORDS.--WSP 805, WDR WI-73-1: Drainage area.

GAGE.--Water-stage recorder. Datum of gage is $840.00 \mathrm{ft}$ above NGVD of 1929 (levels by Wisconsin Department of Natural Resources).

REMARKS.--Lake level regulated by dams at outlets of Lake Mendota and Lake Waubesa. Gage-height telemeter at station.

EXTREMES FOR PERIOD OF RECORD.--Maximum gage height observed, $7.22 \mathrm{ft}$, June 15-17, 2008; minimum observed, $3.50 \mathrm{ft}$, Feb.14, 2006, current datum.

EXTREMES FOR CURRENT YEAR.--Maximum recorded gage height, 5.95 ft, Aug. 27; minimum recorded, 3.99 ft, Apr. 12. 
05429485 LAKE WAUBESA AT MCFARLAND, WI

GAGE HEIGHT, FEET

WATER YEAR OCTOBER 2013 TO SEPTEMBER 2014 DAILY MEAN VALUES

[e, estimated]

\begin{tabular}{|c|c|c|c|c|c|c|c|c|c|c|c|c|}
\hline DAY & OCT & NOV & DEC & JAN & FEB & MAR & APR & MAY & JUN & JUL & AUG & SEP \\
\hline 1 & 4.85 & 4.62 & 4.81 & 4.24 & 4.10 & 4.14 & $\mathrm{e} 4.20$ & 4.88 & 4.84 & 5.79 & 5.50 & 5.78 \\
\hline 2 & 4.84 & 4.63 & 4.81 & 4.23 & 4.11 & 4.13 & $\mathrm{e} 4.18$ & 4.86 & 5.01 & 5.83 & 5.53 & 5.76 \\
\hline 4 & 4.82 & 4.60 & 4.83 & 4.20 & 4.11 & 4.12 & 4.14 & 4.82 & 5.17 & 5.77 & 5.58 & 5.78 \\
\hline 5 & 4.83 & 4.62 & 4.73 & 4.19 & 4.12 & 4.13 & 4.13 & 4.78 & 5.17 & 5.73 & 5.67 & 5.83 \\
\hline 6 & 4.84 & 4.69 & 4.81 & e4.19 & 4.13 & 4.12 & 4.10 & 4.74 & 5.14 & 5.71 & 5.70 & 5.79 \\
\hline 7 & 4.82 & 4.72 & 4.79 & e4.18 & 4.13 & 4.11 & 4.09 & 4.72 & 5.12 & 5.71 & 5.71 & 5.74 \\
\hline 8 & 4.78 & 4.71 & 4.71 & 4.17 & 4.14 & 4.10 & 4.10 & 4.73 & 5.11 & 5.72 & 5.71 & 5.71 \\
\hline 9 & 4.76 & 4.70 & 4.67 & 4.18 & 4.14 & 4.10 & 4.05 & 4.73 & 5.09 & 5.69 & 5.71 & 5.66 \\
\hline 10 & 4.74 & 4.70 & 4.63 & 4.17 & 4.14 & 4.10 & 4.02 & 4.70 & 5.07 & 5.65 & 5.72 & 5.68 \\
\hline 11 & 4.73 & 4.70 & 4.59 & 4.19 & 4.14 & 4.12 & 4.01 & 4.67 & 5.06 & 5.62 & 5.73 & 5.64 \\
\hline 12 & 4.71 & 4.68 & 4.55 & 4.19 & 4.14 & 4.16 & 3.99 & 4.73 & 5.05 & 5.60 & 5.76 & 5.60 \\
\hline 14 & 4.69 & 4.62 & 4.49 & 4.20 & 4.15 & 4.18 & 4.42 & 4.84 & 4.99 & 5.60 & 5.73 & 5.49 \\
\hline 15 & 4.69 & 4.62 & 4.46 & 4.20 & 4.16 & 4.25 & 4.54 & 4.83 & 4.95 & 5.58 & 5.70 & 5.46 \\
\hline 16 & 4.70 & 4.63 & 4.44 & 4.21 & 4.16 & 4.26 & 4.55 & 4.81 & 4.95 & 5.56 & 5.67 & 5.43 \\
\hline 17 & 4.71 & 4.72 & 4.42 & 4.20 & 4.18 & 4.25 & 4.56 & 4.79 & 5.04 & 5.54 & 5.66 & 5.38 \\
\hline 18 & 4.71 & 4.83 & 4.39 & 4.20 & 4.19 & 4.23 & 4.59 & 4.75 & 5.12 & 5.52 & 5.64 & 5.34 \\
\hline 19 & 4.69 & 4.83 & 4.37 & 4.19 & 4.19 & 4.27 & 4.59 & 4.71 & 5.23 & 5.53 & 5.67 & 5.30 \\
\hline 20 & 4.67 & 4.81 & 4.36 & 4.18 & 4.21 & 4.30 & 4.59 & 4.70 & 5.49 & 5.54 & 5.68 & 5.27 \\
\hline 21 & 4.66 & 4.83 & 4.34 & 4.17 & 4.24 & 4.30 & 4.63 & 4.69 & 5.63 & 5.55 & 5.71 & 5.27 \\
\hline 22 & 4.64 & 4.85 & 4.36 & 4.16 & 4.23 & 4.31 & 4.66 & 4.67 & 5.66 & 5.55 & 5.75 & 5.23 \\
\hline 23 & 4.62 & 4.85 & 4.35 & 4.15 & 4.22 & 4.29 & 4.65 & 4.63 & 5.67 & 5.55 & 5.75 & 5.19 \\
\hline 24 & 4.61 & 4.85 & 4.34 & 4.14 & 4.21 & 4.28 & 4.65 & 4.60 & 5.66 & 5.53 & 5.74 & 5.16 \\
\hline 25 & 4.57 & 4.83 & 4.32 & 4.13 & 4.19 & 4.27 & 4.71 & 4.59 & 5.62 & 5.52 & 5.84 & 5.13 \\
\hline 26 & 4.53 & 4.80 & 4.31 & 4.13 & 4.17 & 4.24 & 4.72 & 4.59 & 5.56 & 5.52 & 5.94 & 5.10 \\
\hline 28 & 4.49 & 4.84 & 4.28 & 4.12 & 4.15 & $\mathrm{e} 4.24$ & 4.73 & 4.88 & 5.45 & 5.51 & 5.91 & 5.06 \\
\hline 29 & 4.48 & 4.83 & 4.27 & 4.11 & --- & $\mathrm{e} 4.24$ & 4.83 & 4.92 & 5.44 & 5.50 & 5.87 & 5.03 \\
\hline 30 & 4.48 & 4.82 & 4.25 & 4.10 & --- & $\mathrm{e} 4.23$ & 4.88 & 4.90 & 5.59 & 5.50 & 5.84 & 5.01 \\
\hline 31 & 4.54 & -- & 4.24 & 4.10 & --- & 4.23 & --- & 4.87 & --- & 5.50 & 5.81 & --- \\
\hline MEAN & 4.69 & 4.74 & 4.50 & 4.17 & 4.16 & 4.20 & 4.41 & 4.76 & 5.25 & 5.61 & 5.72 & 5.44 \\
\hline MAX & 4.85 & 4.86 & 4.83 & 4.24 & 4.24 & 4.31 & 4.88 & 4.92 & 5.67 & 5.83 & 5.95 & 5.83 \\
\hline MIN & 4.48 & 4.60 & 4.24 & 4.10 & 4.10 & 4.10 & 3.99 & 4.59 & 4.84 & 5.50 & 5.50 & 5.01 \\
\hline
\end{tabular}




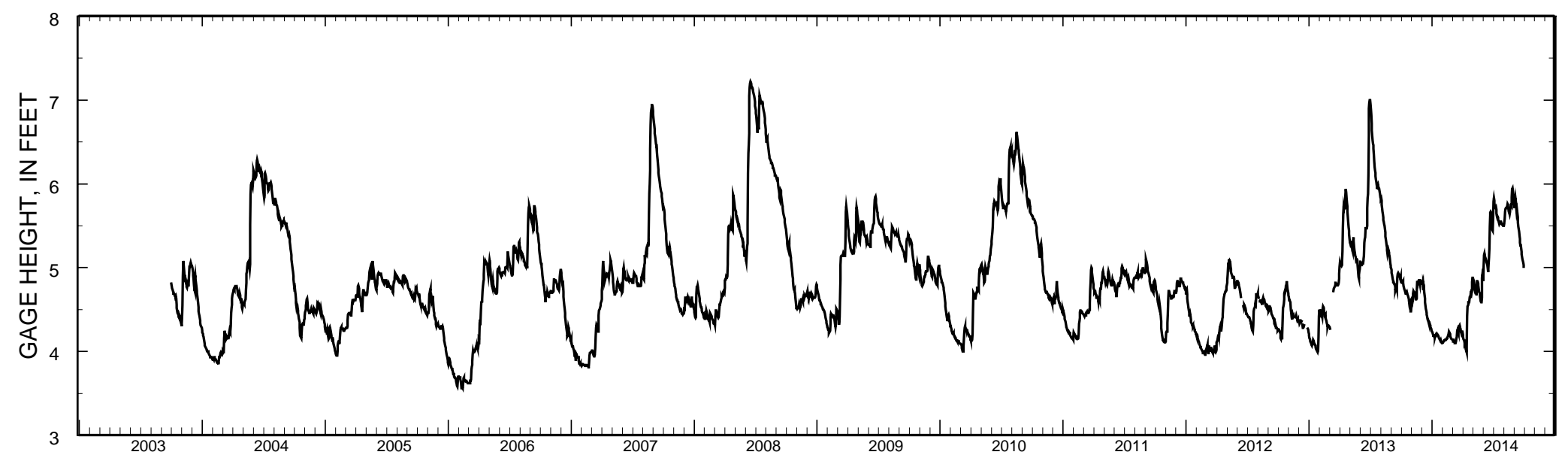

Stage hydrograph for Lake Waubesa, 2003-2014. 


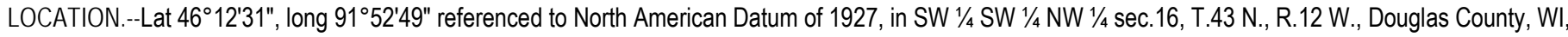
Hydrologic Unit 07030002.

DRAINAGE AREA.--0.81 $\mathrm{mi}^{2}$

PERIOD OF RECORD.--October 2011 to present. Archived data from 2004 to September 2011.

GAGE.--Non-recording gage. Steel rod pounded into lake bed. The rod is installed/removed seasonally to coincide with measuring stage during the open water period.

COOPERATION.--Wisconsin Department of Natural Resources.

REMARKS.--Water surface elevation collected by land owner (observer).

EXTREMES FOR PERIOD OF RECORD.--Maximum daily gage height, 5.90 ft, Sept. 13, 2014; Minimum daily gage height, 3.75 ft, October $22,2012$.

EXTREMES FOR CURRENT YEAR.--Maximum daily gage height, 5.90 ft, Sept. 13; Minimum daily gage height, $4.80 \mathrm{ft}$, Nov. 7, 2013.

GAGE HEIGHT, FEET

WATER YEAR OCTOBER 2013 TO SEPTEMBER 2014

\begin{tabular}{|c|c|c|c|c|c|c|c|c|c|c|c|c|}
\hline DAY & ОСТ & NOV & DEC & JAN & FEB & MAR & APR & MAY & JUN & JUL & AUG & SEP \\
\hline 1 & --- & --- & --- & --- & $\ldots$ & --- & --- & --- & 5.75 & --- & --- & --- \\
\hline 2 & --. & -.- & --- & --- & $-\ldots$ & --- & --- & -.- & --- & --- & --- & --- \\
\hline 3 & --- & --- & --- & --- & --- & --- & --- & $-\ldots$ & --- & --- & --- & --- \\
\hline 4 & --- & --- & --- & --- & $\ldots$ & --- & --- & --- & --- & --- & --- & --- \\
\hline 5 & --- & --- & --- & --- & $-\ldots$ & --- & --- & --- & --- & --- & $\ldots$ & --- \\
\hline 6 & --- & --- & --- & --- & --- & --- & --- & --- & --- & --- & --- & --- \\
\hline 7 & --- & 4.80 & --- & --- & 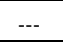 & --- & --- & --- & --- & --- & --- & --- \\
\hline 8 & --- & $-\cdots$ & --- & --- & $-\ldots$ & --- & --- & $\ldots$ & --- & --- & --- & --- \\
\hline 9 & --- & --. & --- & --- & -- & --- & --- & $-\ldots$ & --- & 5.63 & --- & --- \\
\hline 10 & --- & --- & --- & --- & --- & --- & --- & --- & --- & --- & --- & --- \\
\hline 11 & --- & --- & $\ldots$ & --- & --- & --- & --- & $-\ldots-$ & --- & --- & 5.47 & --- \\
\hline 12 & --- & --- & --- & --- & --- & --- & --- & --- & --- & --- & --- & --- \\
\hline 13 & --- & --- & --- & --- & --- & --- & --- & --- & --- & --- & --- & 5.90 \\
\hline 14 & --- & --- & --- & --- & 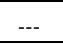 & --- & --- & $\ldots$ & --- & --- & --- & --- \\
\hline 15 & --- & --- & --- & --- & --- & --- & --- & --- & --- & --- & --- & --- \\
\hline 16 & --- & --- & --- & --- & --- & --- & --- & --- & 5.80 & --- & --- & --- \\
\hline 17 & --- & --- & --- & --- & 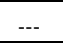 & --- & --- & --- & --- & 5.57 & --- & --- \\
\hline 18 & --- & --- & --- & --- & --- & --- & --- & $\ldots$ & --- & --- & --- & --- \\
\hline 19 & --- & --- & --- & --- & --- & --- & --- & $\ldots$ & --- & --- & --- & --- \\
\hline 20 & --- & --- & --- & --- & --- & --- & --- & --- & --- & --- & --- & --- \\
\hline 21 & --- & $\begin{array}{l}-- \\
\end{array}$ & --- & --- & --- & --- & --- & --- & --- & --- & --- & --- \\
\hline 22 & --- & $-\cdots$ & --- & --- & --- & --- & --- & $-\ldots-$ & --- & --- & --- & --- \\
\hline 23 & --- & --- & --- & --- & --- & --- & --- & --- & 5.71 & 5.47 & --- & --- \\
\hline 24 & --- & --- & --- & --- & --- & --- & --- & --- & --- & --- & --- & --- \\
\hline 25 & --- & --- & --- & --- & --- & --- & --- & --- & --- & --- & 5.50 & --- \\
\hline 26 & --- & --- & --- & --- & --- & --- & --- & --- & --- & --- & 5.63 & --- \\
\hline 27 & --- & -.- & 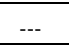 & --- & --- & --- & 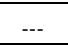 & $-\cdots$ & --- & --- & --- & 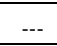 \\
\hline 28 & --- & --- & --- & --- & --- & --- & --- & --- & --- & --- & --- & --- \\
\hline 29 & --- & --- & --- & --- & --- & --- & --- & 5.74 & --- & --- & --- & --- \\
\hline 30 & $\ldots$ & $\ldots$ & $\ldots$ & $\ldots$ & --- & -.- & $\ldots$ & $\ldots$ & $\ldots$ & $\ldots$ & --- & $\ldots$ \\
\hline 31 & --- & --- & --- & --- & --- & --- & --- & --- & --- & --- & --- & --- \\
\hline
\end{tabular}




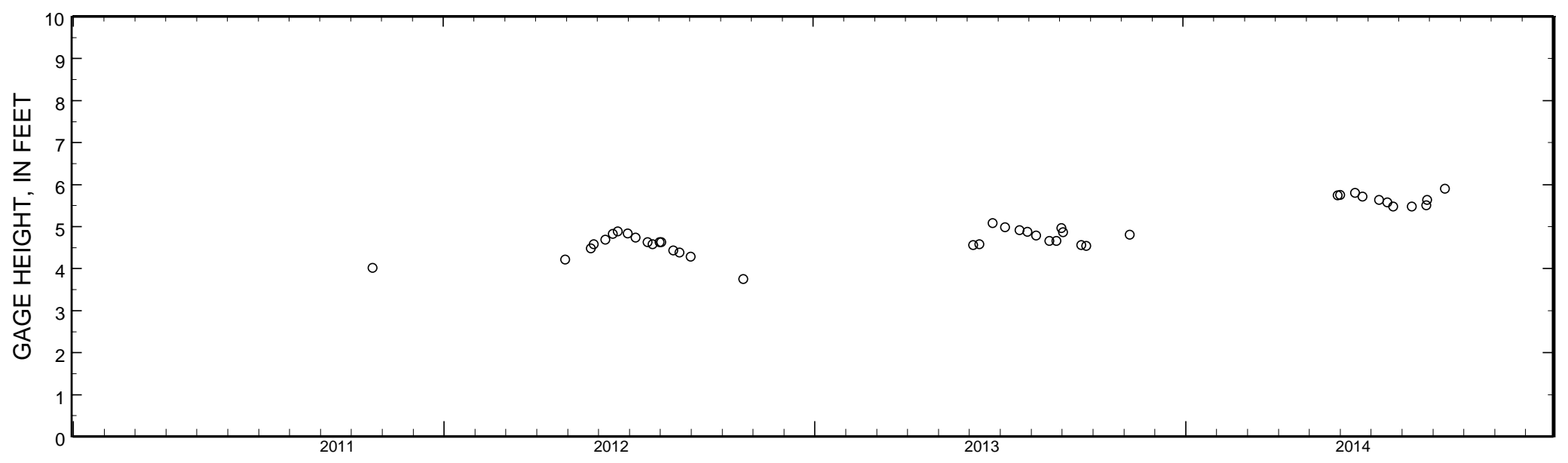

Stage hydrograph for Whitefish (Bardon) Lake near Gordon, 2011-2014. 


\section{WIND LAKE, HEADWATER, AT OUTLET AT WIND LAKE, WI}

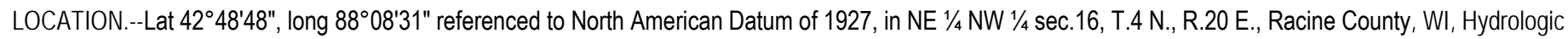
Unit 07120006, at Wind Lake.

SURFACE AREA.--1.46 mi².

DRAINAGE AREA.--39.6 mi².

PERIOD OF RECORD.--March 1985 to current year. Prior to October 2000, published as "Wind Lake Outlet".

REVISED RECORDS.--WDR WI-91-1: 1988(m).

REMARKS.--Lake level regulated by dam with two 10-foot gates at outlet. Lake ice-covered Jan. 14 to Mar. 8. Prior to October 1987, published as Wind Lake at Wind Lake, Wis. Gage-height telemeter at station.

EXTREMES FOR PERIOD OF RECORD.--Maximum gage height, 9.88 ft, June 14, 15, 2008; minimum recorded, 5.95 ft, Jan. 2, 1996.

EXTREMES FOR CURRENT YEAR.-Headwater: Maximum recorded gage height, 8.53 ft, Jul. 13; minimum recorded, 7.63ft, Oct. $25-26$.

\section{GAGE HEIGHT, FEET \\ WATER YEAR OCTOBER 2013 TO SEPTEMBER 2014 \\ DAILY MEAN VALUES}

[e, estimated]

\begin{tabular}{|c|c|c|c|c|c|c|c|c|c|c|c|c|}
\hline DAY & OCT & NOV & DEC & JAN & FEB & MAR & APR & MAY & JUN & JUL & AUG & SEP \\
\hline 1 & 7.77 & 7.78 & 7.73 & 7.72 & 7.70 & 7.68 & 8.18 & 8.17 & 8.25 & 8.26 & 8.30 & 8.28 \\
\hline 2 & 7.76 & 7.72 & 7.72 & 7.73 & 7.71 & 7.70 & 8.20 & 8.19 & 8.35 & 8.25 & 8.39 & 8.30 \\
\hline 3 & 7.75 & 7.68 & 7.72 & 7.73 & 7.72 & 7.71 & 8.20 & 8.20 & 8.35 & 8.27 & 8.36 & 8.27 \\
\hline 5 & 7.79 & 7.71 & 7.73 & 7.75 & 7.76 & 7.75 & 8.16 & 8.22 & 8.29 & 8.26 & 8.33 & 8.32 \\
\hline 6 & 7.86 & 7.74 & 7.72 & 7.74 & 7.76 & 7.76 & 8.14 & 8.23 & 8.29 & 8.27 & 8.31 & 8.27 \\
\hline 9 & 7.80 & 7.70 & 7.69 & 7.73 & 7.75 & 7.79 & 8.28 & 8.22 & 8.29 & 8.38 & 8.28 & 8.24 \\
\hline 10 & 7.78 & 7.68 & 7.69 & 7.74 & 7.75 & 7.81 & 8.23 & 8.23 & 8.28 & 8.35 & 8.27 & 8.23 \\
\hline 11 & 7.75 & 7.70 & 7.69 & 7.77 & 7.74 & 7.85 & 8.23 & 8.22 & 8.34 & 8.32 & 8.35 & 8.22 \\
\hline 12 & 7.74 & 7.73 & 7.69 & 7.77 & 7.73 & 7.90 & 8.20 & 8.23 & 8.33 & 8.33 & 8.36 & 8.23 \\
\hline 13 & 7.73 & 7.73 & 7.69 & 7.77 & 7.72 & 7.94 & 8.21 & 8.31 & 8.30 & 8.53 & 8.34 & 8.23 \\
\hline 18 & 7.66 & 7.88 & 7.71 & 7.72 & 7.69 & 8.25 & 8.17 & 8.27 & 8.25 & 8.32 & 8.29 & 8.25 \\
\hline 19 & 7.64 & 7.93 & 7.72 & 7.72 & 7.69 & 8.18 & 8.14 & 8.28 & 8.23 & 8.31 & 8.43 & 8.24 \\
\hline 20 & 7.64 & 7.89 & 7.72 & 7.72 & 7.72 & 8.13 & 8.15 & 8.26 & 8.23 & 8.34 & 8.42 & 8.23 \\
\hline 21 & 7.64 & 7.77 & 7.73 & 7.72 & 7.73 & 8.14 & 8.16 & 8.25 & 8.22 & 8.34 & 8.36 & 8.27 \\
\hline 22 & 7.63 & 7.65 & 7.77 & 7.71 & 7.71 & 8.15 & 8.18 & 8.24 & 8.22 & 8.31 & 8.34 & 8.25 \\
\hline 23 & 7.64 & 7.70 & 7.77 & 7.71 & 7.68 & 8.13 & 8.17 & 8.23 & 8.26 & 8.31 & 8.33 & 8.25 \\
\hline 24 & 7.64 & 7.70 & 7.75 & 7.70 & 7.67 & 8.12 & 8.17 & 8.22 & 8.36 & 8.30 & 8.37 & 8.24 \\
\hline 25 & 7.63 & 7.73 & 7.74 & 7.70 & 7.66 & 8.12 & 8.18 & 8.21 & 8.33 & 8.30 & 8.41 & 8.24 \\
\hline 26 & 7.63 & 7.76 & 7.72 & 7.70 & 7.65 & 8.10 & 8.18 & 8.21 & 8.25 & 8.30 & 8.40 & 8.24 \\
\hline 27 & 7.64 & 7.76 & 7.71 & 7.70 & 7.66 & 8.10 & 8.17 & 8.23 & 8.23 & 8.31 & 8.37 & 8.24 \\
\hline
\end{tabular}




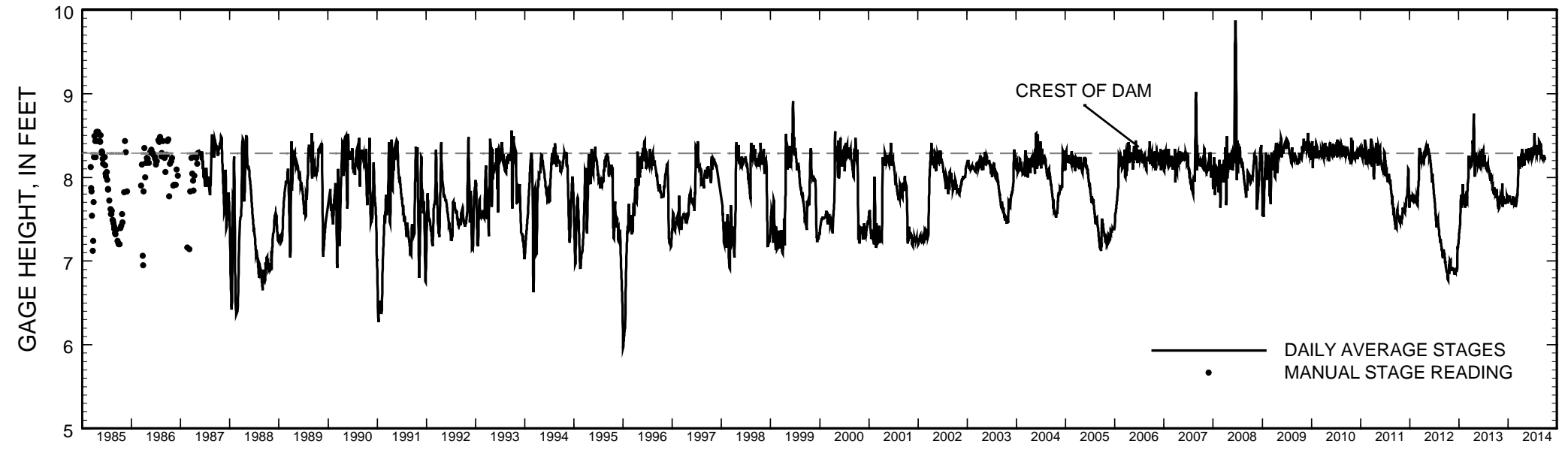

Stage hydrograph for Wind Lake, headwater, 1985-2014. 
424915088083900 WIND LAKE AT WIND LAKE, WI

LOCATION.--Lat 4249'15", long 8808'39", in NW 1/4 SW 1/4 Sec.9, T.4 N., R.20 E., Racine County, Hydrologic Unit 07120006, at Wind Lake.

SURFACE AREA.--1.46 mi².

PERIOD OF RECORD.--February 1985 to current year.

REMARKS.--Lake sampled near center at the deep hole. Water-quality analyses done by Wisconsin State Laboratory of Hygiene.

WATER-QUALITY DATA, MARCH 4 TO AUGUST 27, 2014

(Milligrams per liter unless otherwise indicated)

\begin{tabular}{|c|c|c|c|c|c|c|c|c|c|c|c|c|c|}
\hline Date & $\begin{array}{c}\text { Trans- } \\
\text { parency } \\
\text { Secchi } \\
\text { disc, } \\
\text { meters } \\
(\text { } \odot \circ 78)\end{array}$ & $\begin{array}{c}\text { Sam- } \\
\text { pling } \\
\text { depth, } \\
\text { meters } \\
(\odot \odot \odot 98)\end{array}$ & $\begin{array}{c}\text { Temper - } \\
\text { ature, } \\
\text { water, } \\
\text { deg C } \\
(\odot \odot \odot 10)\end{array}$ & $\begin{array}{c}\text { Specif- } \\
\text { ic } \\
\text { conduc- } \\
\text { tance, } \\
\text { wat unf } \\
\text { uS } / \mathrm{cm} @ \\
25 \mathrm{degc} \\
(\odot \odot \odot 95)\end{array}$ & $\begin{array}{c}\text { pH, } \\
\text { water, } \\
\text { unfltrd } \\
\text { field, } \\
\text { std } \\
\text { units } \\
(\odot \odot 4 \odot \odot)\end{array}$ & $\begin{array}{c}\text { Dis- } \\
\text { solved } \\
\text { oxygen, } \\
\text { mg/L } \\
(\odot \odot 30 \odot)\end{array}$ & $\begin{array}{c}\text { Chloro- } \\
\text { phyll a } \\
\text { trichro } \\
\text {-matic } \\
\text { method, } \\
\text { uncorr, } \\
\text { ug/L } \\
(32210)\end{array}$ & $\begin{array}{c}\text { Phos- } \\
\text { phorus, } \\
\text { water, } \\
\text { unfltrd } \\
\text { mg/L } \\
\text { as P } \\
(00665)\end{array}$ & $\begin{array}{c}\text { Ortho- } \\
\text { phos- } \\
\text { phate, } \\
\text { water, } \\
\text { fltrd, } \\
\text { mg/L } \\
\text { as P } \\
(00671)\end{array}$ & $\begin{array}{c}\text { Total } \\
\text { nitro- } \\
\text { gen, } \\
\text { water, } \\
\text { unfltrd } \\
\text { mg/L } \\
(0060 \odot)\end{array}$ & $\begin{array}{c}\text { Ammonia } \\
\text { water, } \\
\text { fltrd, } \\
\text { mg/L } \\
\text { as N } \\
(\odot \odot 6 \odot 8)\end{array}$ & $\begin{array}{c}\text { Ammonia } \\
+ \\
\text { org-N, } \\
\text { water, } \\
\text { fltrd, } \\
\text { mg/L } \\
\text { as N } \\
(00623)\end{array}$ & $\begin{array}{c}\text { Ammonia } \\
+ \\
\text { org-N, } \\
\text { water, } \\
\text { unfltrd } \\
\text { mg/L } \\
\text { as N } \\
(00625)\end{array}$ \\
\hline
\end{tabular}

\begin{tabular}{|c|c|c|c|c|c|c|c|c|c|c|c|c|c|}
\hline \multicolumn{14}{|l|}{ MAR 2014} \\
\hline $04 \ldots$ & -- & 1.0 & 2.5 & 863 & 7.9 & 11.1 & 4.74 & $\odot .021$ & - - & - - & -- & -- & -- \\
\hline$\odot 4 \ldots$ & -- & 14.0 & 2.5 & 1340 & 7.5 & 3.9 & - - & $\odot .023$ & -- & - - & - - & -- & -- \\
\hline \multicolumn{14}{|l|}{ MAY } \\
\hline $09 \ldots$ & 4.05 & -- & - - & - - & -- & - - & - - & - - & - - & - - & - - & -- & -- \\
\hline $09 \ldots$ & - - & 0.50 & 13.7 & 805 & 8.1 & 10.0 & 2.21 & $\odot .021$ & $\odot . \odot \odot 2$ & 1.2 & $\odot .067$ & -- & 1.0 \\
\hline $16 \ldots$ & 3.80 & - - & - - & - - & - & - - & - & - - & -- & - - & -- & - & -- \\
\hline $16 \ldots$ & -- & 0.50 & 22.2 & 801 & 8.6 & 9.3 & 4.77 & $\odot .150$ & - - & - - & - - & - - & 1.7 \\
\hline $16 \ldots$ & -- & 13.5 & 12.7 & 839 & 7.4 & 0.2 & -- & $\odot .021$ & -- & - - & -- & -- & 0.88 \\
\hline \multicolumn{14}{|l|}{ JUL } \\
\hline $29 \ldots$ & 2.30 & - - & - - & - - & -- & - - & - & - - & -- & - - & -- & - & - - \\
\hline $29 \ldots$ & -- & 13.5 & 13.0 & 826 & 7.2 & 0.2 & - - & $\odot .154$ & -- & - - & -- & -- & -- \\
\hline \multicolumn{14}{|l|}{ AUG } \\
\hline $27 \ldots$ & 2.50 & - - & - - & -- & -- & - - & -- & - - & -- & - - & -- & -- & - - \\
\hline $27 \ldots$ & -- & 0.50 & 25.6 & 744 & 8.5 & 8.3 & 8.47 & $\odot .025$ & -- & - - & -- & -- & - - \\
\hline $27 \ldots$ & -- & 6.0 & 22.0 & 760 & 7.8 & 0.8 & - - & $\odot .024$ & -- & - - & -- & -- & - - \\
\hline $27 \ldots$ & -- & 10.0 & 14.5 & 839 & 7.6 & 0.3 & -- & $\odot .149$ & -- & - - & -- & -- & - - \\
\hline $27 \ldots$ & - - & 12.0 & 13.7 & 843 & 7.5 & 0.3 & - - & $\odot .159$ & -- & - - & -- & -- & -- \\
\hline $27 \ldots$ & - - & 13.5 & 13.4 & 845 & 7.4 & 0.2 & - - & 0.201 & -- & - - & - - & -- & -- \\
\hline
\end{tabular}


424915088083900 WIND LAKE AT WIND LAKE, WI

WATER-QUALITY DATA, MARCH 4 TO AUGUST 27, 2014

(Milligrams per liter unless otherwise indicated)

\begin{tabular}{|c|c|c|c|c|c|c|c|c|c|c|c|c|c|}
\hline Date & $\begin{array}{c}\text { Nitrate } \\
+ \\
\text { nitrite } \\
\text { water, } \\
\text { fltrd, } \\
\text { mg/L } \\
\text { as N } \\
(00631)\end{array}$ & $\begin{array}{c}\text { Turbdty } \\
\text { white } \\
\text { light, } \\
\text { det ang } \\
90+/-30 \\
\text { degrees } \\
\text { NTU } \\
(63675)\end{array}$ & $\begin{array}{l}\text { Appar- } \\
\text { ent } \\
\text { color, } \\
\text { water, } \\
\text { unfltrd } \\
\text { Pt-Co } \\
\text { units } \\
(00081)\end{array}$ & $\begin{array}{c}\text { Hard- } \\
\text { ness, } \\
\text { water, } \\
\mathrm{mg} / \mathrm{L} \text { as } \\
\text { Cac03 } \\
(\odot \odot 90 \odot)\end{array}$ & $\begin{array}{c}\text { Calcium } \\
\text { water, } \\
\text { fltrd, } \\
\text { mg/L } \\
(00915)\end{array}$ & $\begin{array}{c}\text { Magnes- } \\
\text { ium, } \\
\text { water, } \\
\text { fltrd, } \\
\text { mg/L } \\
(00925)\end{array}$ & $\begin{array}{c}\text { Sodium, } \\
\text { water, } \\
\text { fltrd, } \\
\text { mg/L } \\
(00930)\end{array}$ & $\begin{array}{c}\text { Potas- } \\
\text { sium, } \\
\text { water, } \\
\text { fltrd, } \\
\text { mg/L } \\
(00935)\end{array}$ & $\begin{array}{c}\text { ANC, } \\
\text { wat unf } \\
\text { fixed } \\
\text { end pt, } \\
\text { lab, } \\
\text { mg/L as } \\
\text { Caco3 } \\
(0 \odot 417)\end{array}$ & $\begin{array}{c}\text { Chlor - } \\
\text { ide, } \\
\text { water, } \\
\text { fltrd, } \\
\text { mg/L } \\
(00940)\end{array}$ & $\begin{array}{c}\text { Sulfate } \\
\text { water, } \\
\text { fltrd, } \\
\text { mg/L } \\
(00945)\end{array}$ & $\begin{array}{c}\text { Silica, } \\
\text { water, } \\
\text { fltrd, } \\
\text { mg/L as } \\
\text { Si02 } \\
(00955)\end{array}$ & $\begin{array}{c}\text { Iron, } \\
\text { water, } \\
\text { fltrd, } \\
\text { ug/L } \\
(01046)\end{array}$ \\
\hline
\end{tabular}

MAR 2014

$\odot 4 \ldots$
$\odot 4 \ldots$
MAY
$\odot 9 \ldots$
$09 \ldots$
JUN
$16 \ldots$
$16 \ldots$
$16 \ldots$
JUL
$29 \ldots$
$29 \ldots$
$29 \ldots$
AUG
$27 \ldots$
$27 \ldots$
$27 \ldots$
$27 \ldots$
$27 \ldots$
$27 \ldots$

$\begin{array}{ccc} & & \\ -- & -- & -- \\ -- & -- & -- \\ -- & -- & -- \\ 228 & 49.7 & 25.3 \\ & & \\ -- & -- & -- \\ -- & -- & -- \\ -- & -- & -- \\ & & \\ -- & -- & - \\ -- & -- & - \\ -- & -- & - \\ & & \\ -- & -- & -- \\ -- & -- & -- \\ -- & -- & -- \\ -- & -- & -- \\ -- & -- & -- \\ -- & -- & --\end{array}$

\begin{tabular}{ccc}
-- & -- & -- \\
0.198 & -- & -- \\
-- & -- & - \\
-- & 1.7 & 30 \\
-- & -- & -- \\
-- & -- & -- \\
\hdashline 0.019 & -- & -- \\
-- & -- & -- \\
-- & -- & -- \\
-- & -- & - \\
-- & -- & -- \\
-- & -- & -- \\
-- & -- & -- \\
-- & -- & --
\end{tabular}

$\begin{array}{ccc} & \begin{array}{c}\text { Dis- } \\ \text { solved } \\ \text { solids } \\ \text { Manga- } \\ \text { Date }\end{array} \\ \text { nese, } & \text { dried @ } \\ \text { water, } & 180 \text { degC } \\ \text { fltrd, } & \text { wat flt } \\ \text { ug/L } & \mathrm{mg} / \mathrm{L} \\ (01056) & (70300)\end{array}$

MAY
O9...
JUN
$16 \ldots$

$<1.0$

- 430 
424915088083900 WIND LAKE AT WIND LAKE, WI

LAKE-DEPTH PROFILES, MARCH 4 TO AUGUST 27, 2014

03-04-14

05-09-14

06-16-14

07-29-14

08-27-14

DISSOLVED OXYGEN (D.O.), IN MILLIGRAMS PER LITER
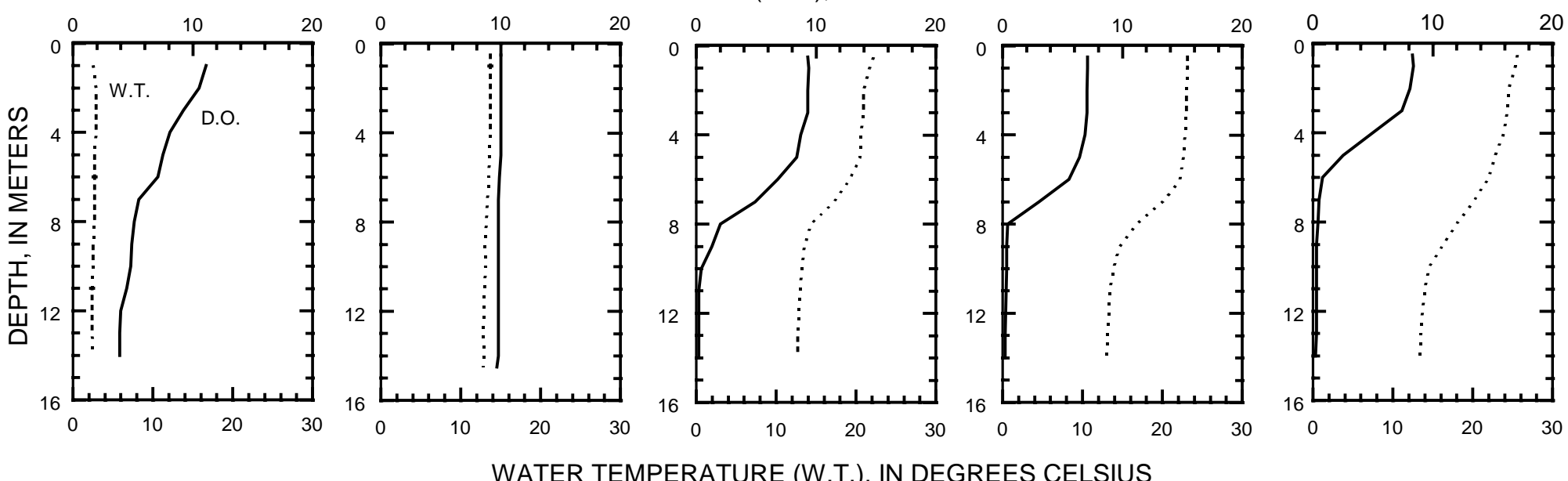

WATER TEMPERATURE (W.T.), IN DEGREES CELSIUS
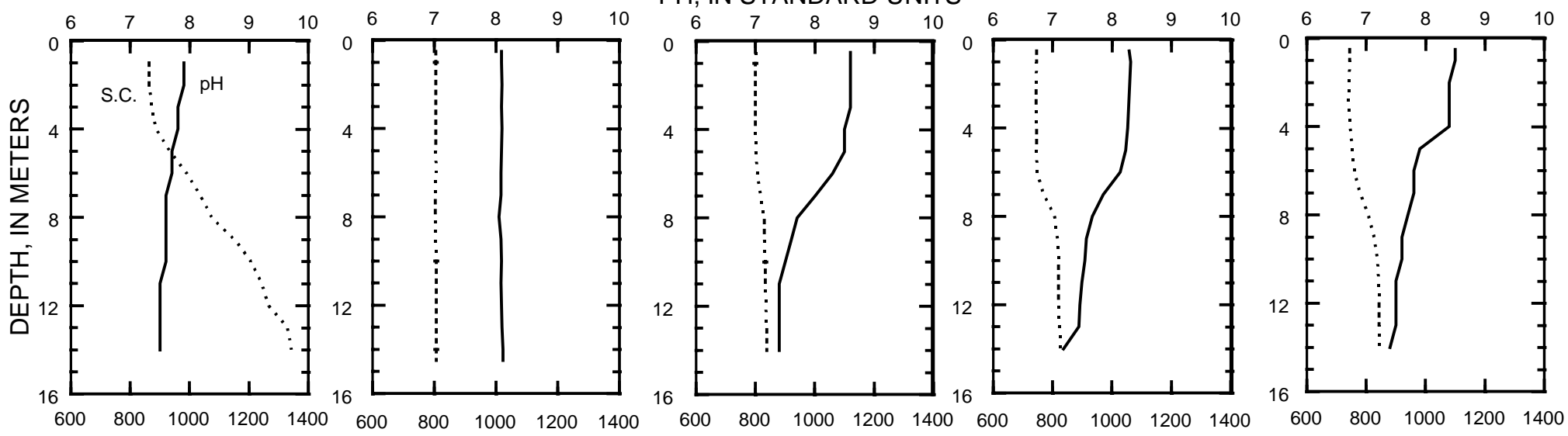

SPECIFIC CONDUCTANCE (S.C.), IN MICROSIEMENS PER CENTIMETER AT 25 DEGREES CELSIUS 

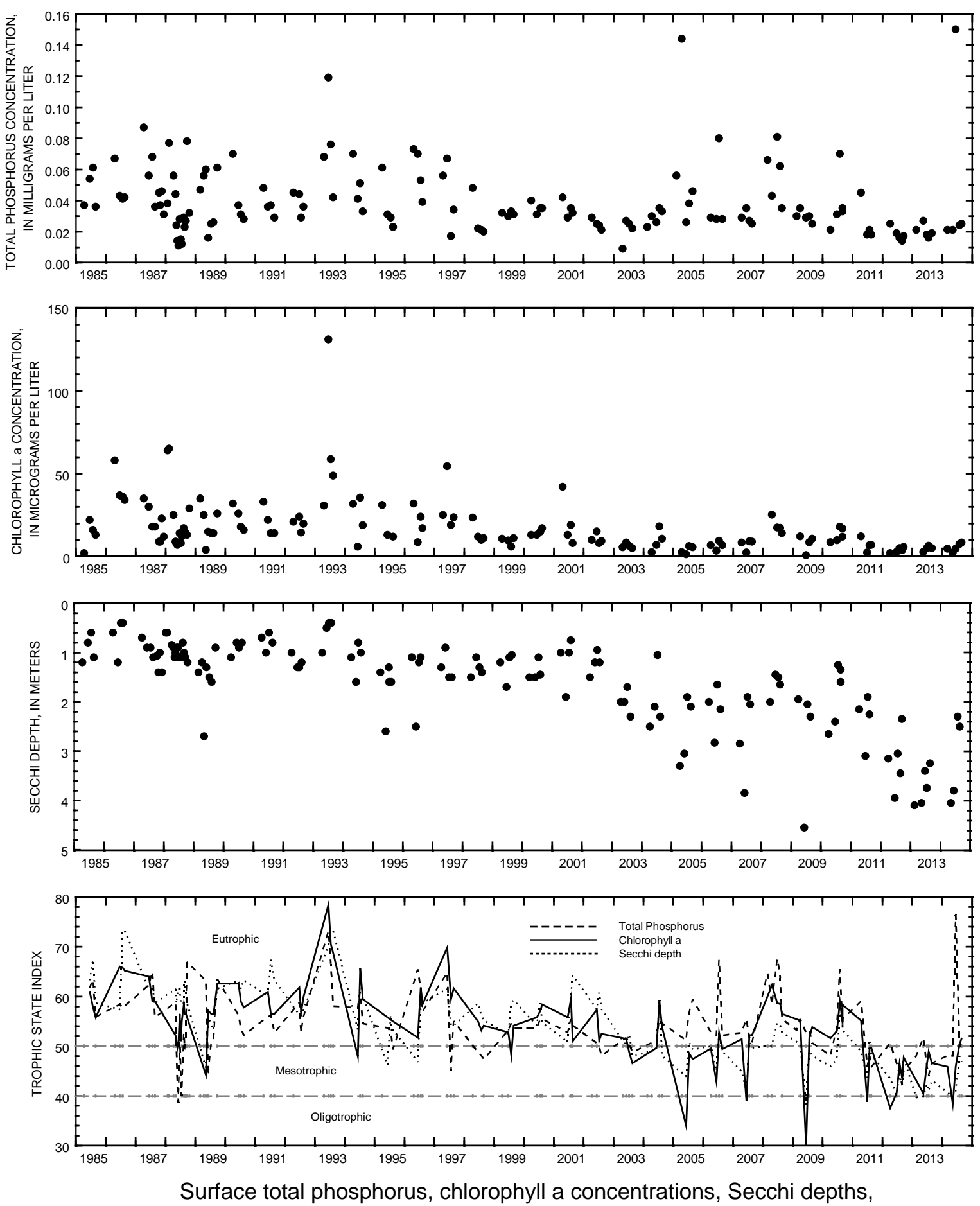

and TSI data for Wind Lake, Deep Hole, at Wind Lake, Wisconsin. 


\section{LAKE WINNEBAGO AT OSHKOSH, WI}

LOCATION.--Lat $44^{\circ} 00^{\prime} 35^{\prime \prime}$, long $88^{\circ} 31^{\prime} 38^{\prime \prime}$ referenced to North American Datum of 1927, in NE $1 / 4$ NE $1 / 4$ sec. 25 , T.18 N., R.16 E., Winnebago County, WI, Hydrologic Unit 04030203, $800 \mathrm{ft}$ east of mouth of the upper Fox River.

SURFACE AREA.--215 $\mathrm{mi}^{2}$.

DRAINAGE AREA.--5,880 mi².

PERIOD OF RECORD.--October 1938 to current year in reports of Geological Survey. Records from July 1882 to September 1938 in files of Geological Survey and U.S. Army Corps of Engineers. A report on Fox River by U.S. Army Corps of Engineers, published as House Document No. 146, 67th Congress, 2nd session, contains semimonthly records of inflow of Lake Winnebago for the period 1896-1917.

REVISED RECORDS.--WDR WI-83-1: Drainage area.

GAGE.--Water-stage recorder. Nonrecording gage read once daily October 1938 to October 1978. Datum of gage is $743.91 \mathrm{ft}$ above NAVD of 1988 (Wisconsin Department of Transportation benchmark). From July 1992 to March 20, 2007 datum was $743.97 \mathrm{ft}$ above NAVD of 1988. Prior to 2012 datum of gage was published as $745.05 \mathrm{ft}$ above mean tide at New York City.

REMARKS.--Lake elevations controlled by dams at Menasha and Neenah. Data-collection platform and gage-height telemeter at station.

EXTREMES FOR PERIOD OF RECORD.-- Water year 1938-1992; maximum gage height observed, $4.32 \mathrm{ft}$ Mar. 9, 1982, datum unknown; minimum observed, $0.33 \mathrm{ft}$ May 17, 1960, datum unknown. Water year 1993-current year; maximum daily mean gage height, $3.85 \mathrm{ft}$ June 14, 2008; minimum recorded, $1.15 \mathrm{ft}$ (estimated), March 13-14, 2014.

EXTREMES FOR CURRENT YEAR.--Maximum daily mean gage height, $3.21 \mathrm{ft}$, June 25; Minimum recorded, 1.15 ft (estimated), March $13-14$. 
04082500 LAKE WINNEBAGO AT OSHKOSH, WI

GAGE HEIGHT, FEET

WATER YEAR OCTOBER 2013 TO SEPTEMBER 2014

DAILY MEAN VALUES

[e, estimated]

\begin{tabular}{|c|c|c|c|c|c|c|c|c|c|c|c|c|}
\hline DAY & ОСТ & NOV & DEC & JAN & FEB & MAR & APR & MAY & JUN & JUL & AUG & SEP \\
\hline 1 & 2.68 & 2.48 & 2.36 & 2.01 & 1.74 & 1.35 & 1.49 & 2.69 & 2.94 & 3.04 & 3.00 & 2.90 \\
\hline 2 & 2.70 & 2.50 & 2.34 & 2.01 & 1.73 & 1.33 & 1.51 & 2.69 & 3.13 & 3.10 & 2.99 & 2.89 \\
\hline 3 & 2.70 & 2.50 & 2.33 & 2.00 & 1.72 & 1.31 & 1.52 & 2.68 & 3.16 & 3.12 & 2.99 & 2.88 \\
\hline 4 & 2.74 & 2.46 & 2.33 & 2.00 & 1.70 & 1.29 & 1.54 & 2.68 & 3.20 & 3.11 & 3.03 & 2.88 \\
\hline 5 & 2.74 & 2.42 & 2.27 & 1.98 & 1.69 & 1.28 & 1.57 & 2.70 & 3.16 & 3.06 & 3.03 & 2.86 \\
\hline 6 & 2.74 & 2.48 & 2.27 & 1.98 & 1.68 & 1.26 & 1.58 & 2.68 & 3.13 & 3.07 & 3.03 & 2.90 \\
\hline 7 & 2.73 & 2.49 & 2.27 & 1.97 & 1.67 & 1.25 & 1.58 & 2.71 & 3.11 & 3.08 & 3.02 & 2.92 \\
\hline 8 & 2.71 & 2.48 & 2.24 & 1.95 & 1.65 & 1.23 & 1.60 & 2.69 & 3.15 & 3.12 & 3.00 & 2.91 \\
\hline 9 & 2.66 & 2.41 & 2.23 & 1.94 & 1.63 & 1.21 & 1.61 & 2.66 & 3.11 & 3.14 & 2.99 & 2.92 \\
\hline 10 & 2.64 & 2.41 & 2.20 & 1.94 & 1.59 & e1.19 & 1.62 & 2.69 & 3.10 & 3.14 & 2.97 & 2.90 \\
\hline 11 & 2.63 & 2.43 & 2.17 & 1.94 & 1.57 & e1.17 & 1.62 & 2.74 & 3.11 & 3.08 & 2.97 & 2.94 \\
\hline 12 & 2.60 & 2.41 & 2.14 & 1.94 & 1.56 & e1.16 & 1.66 & 2.88 & 3.09 & 3.08 & 2.99 & 2.94 \\
\hline 13 & 2.60 & 2.38 & 2.11 & 1.93 & 1.56 & e1.15 & 1.88 & 2.87 & 3.08 & 3.09 & 3.00 & 2.95 \\
\hline 14 & 2.63 & 2.35 & 2.09 & 1.93 & 1.55 & e1.15 & 2.18 & 2.90 & 3.09 & 3.07 & 2.98 & 2.92 \\
\hline 15 & 2.64 & 2.37 & 2.08 & 1.92 & 1.54 & e1.16 & 2.30 & 2.94 & 3.02 & 3.08 & 2.96 & 2.95 \\
\hline 16 & 2.63 & 2.37 & 2.06 & 1.92 & 1.52 & $\mathrm{e} 1.17$ & 2.40 & 2.89 & 2.98 & 3.08 & 2.96 & 2.96 \\
\hline 17 & 2.65 & 2.39 & 2.05 & 1.92 & 1.52 & $\mathrm{e} 1.18$ & 2.43 & 2.84 & 3.09 & 3.07 & 2.98 & 2.97 \\
\hline 18 & 2.63 & 2.41 & 2.04 & 1.91 & 1.52 & e1.19 & 2.41 & 2.82 & 3.17 & 3.07 & 2.97 & 3.00 \\
\hline 19 & 2.60 & 2.52 & 2.04 & 1.90 & 1.52 & $\mathrm{e} 1.20$ & 2.48 & 2.82 & 3.18 & 3.04 & 3.06 & 2.96 \\
\hline 20 & 2.58 & 2.49 & 2.03 & 1.89 & 1.51 & e1.22 & 2.50 & 2.80 & 3.16 & 3.06 & 3.10 & 2.96 \\
\hline 21 & 2.51 & 2.48 & 2.03 & 1.88 & 1.51 & 1.24 & 2.54 & 2.79 & 3.17 & 3.05 & 3.12 & 3.01 \\
\hline 22 & 2.51 & 2.48 & 2.03 & 1.88 & 1.50 & 1.25 & 2.57 & 2.82 & 3.15 & 3.04 & 3.12 & 3.02 \\
\hline 23 & 2.48 & 2.48 & 2.04 & 1.87 & 1.48 & 1.28 & 2.62 & 2.86 & 3.13 & 3.07 & 3.12 & 3.01 \\
\hline 24 & 2.45 & 2.47 & 2.04 & 1.86 & 1.46 & 1.30 & 2.66 & 2.88 & 3.16 & 3.03 & 3.12 & 2.99 \\
\hline 25 & 2.42 & 2.38 & 2.05 & 1.85 & 1.43 & 1.32 & 2.65 & 2.90 & 3.21 & 2.99 & 3.11 & 2.96 \\
\hline 26 & 2.36 & 2.39 & 2.05 & 1.84 & 1.41 & 1.33 & 2.64 & 2.92 & 3.18 & 2.99 & 3.12 & 2.95 \\
\hline 27 & 2.38 & 2.38 & 2.04 & 1.82 & 1.39 & 1.34 & 2.70 & 2.98 & 3.16 & 3.00 & 3.13 & 2.92 \\
\hline 28 & 2.40 & 2.39 & 2.04 & 1.80 & 1.36 & 1.44 & 2.70 & 3.00 & 3.12 & 2.98 & 3.08 & 2.90 \\
\hline 29 & 2.38 & 2.38 & 2.04 & 1.78 & --- & 1.45 & 2.70 & 2.99 & 3.07 & 2.97 & 3.00 & 2.89 \\
\hline 30 & 2.35 & 2.37 & 2.03 & 1.76 & --- & 1.45 & 2.69 & 2.98 & 3.07 & 2.99 & 2.97 & 2.86 \\
\hline 31 & 2.43 & --- & 2.02 & 1.75 & --- & 1.47 & --- & 2.95 & --- & 2.98 & 2.97 & 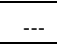 \\
\hline MEAN & 2.58 & 2.43 & 2.13 & 1.91 & 1.56 & 1.27 & 2.13 & 2.82 & 3.12 & 3.06 & 3.03 & 2.93 \\
\hline MAX & 2.74 & 2.52 & 2.36 & 2.01 & 1.74 & 1.47 & 2.70 & 3.00 & 3.21 & 3.14 & 3.13 & 3.02 \\
\hline MIN & 2.35 & 2.35 & 2.02 & 1.75 & 1.36 & 1.15 & 1.49 & 2.66 & 2.94 & 2.97 & 2.96 & 2.86 \\
\hline
\end{tabular}




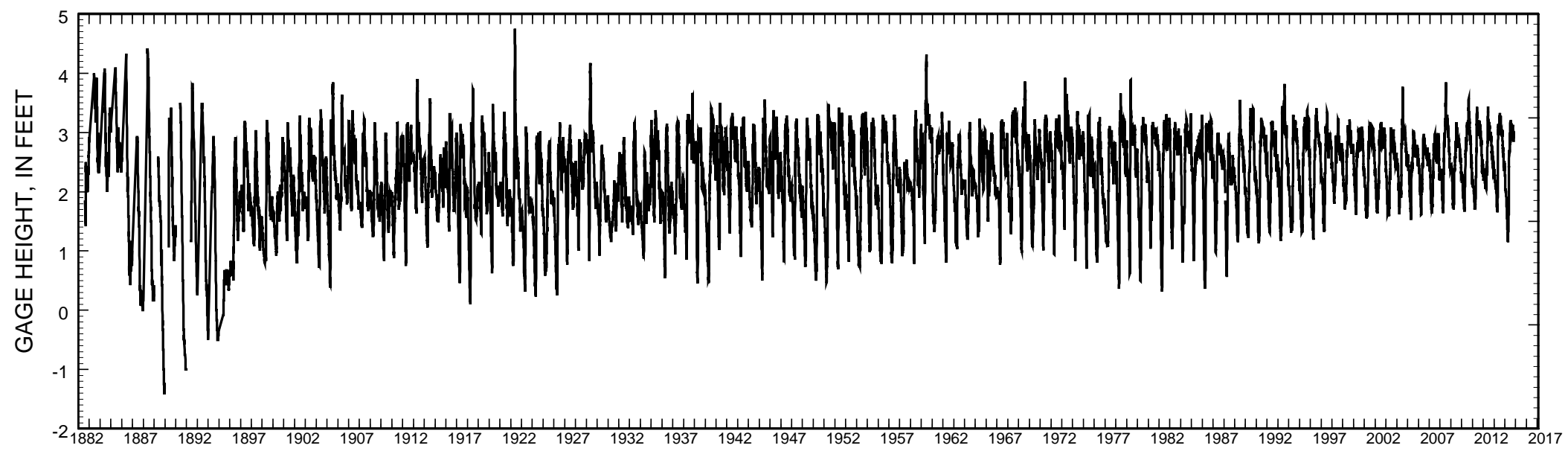

Stage hydrograph for Lake Winnebago at Oshkosh, WI, 1882-2014. 


\section{LAKE WINNEBAGO NEAR STOCKBRIDGE, WI}

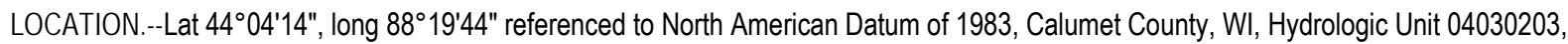
Stockbridge Indian Reservation, on east shore of Lake Winnebago, $300 \mathrm{ft}$ south of County Highway E and 1.6 mi west of Stockbridge.

SURFACE AREA.--215 $\mathrm{mi}^{2}$.

DRAINAGE AREA.--5,880 $\mathrm{mi}^{2}$.

PERIOD OF RECORD.--November 1982 to current year.

GAGE.--Water-stage recorder. Datum of gage from July 1992 to current year is $744.00 \mathrm{ft}$ above NAVD of 1988 (RTK-GPS survey). Prior to 2012 pbulished as $745.05 \mathrm{ft}$ above mean tide of New York City.

REMARKS.--Lake elevations controlled by dams at Menasha and Neenah. Data-collection platform and gage-height telemeter at station.

EXTREMES FOR PERIOD OF RECORD.-- Water year 1982-1992; maximum daily mean gage height, $3.53 \mathrm{ft}$, May 31, 1989, datum unknown; minimum recorded, $0.30 \mathrm{ft}$, Mar. 1, 1986, datum unknown. Water year 1993 to current year; maximum daily mean gage height, $3.85 \mathrm{ft}$, July 9, 11, 1993, June 14, 2008; minimum recorded $1.04 \mathrm{ft}$ (estimated) March 13-14, 2014.

EXTREMES FOR CURRENT YEAR.--Maximum daily mean gage height, $3.18 \mathrm{ft}$, June 3; minimum recorded, $1.04 \mathrm{ft}$ (estimated), Mar. 13-14. 
04084255 LAKE WINNEBAGO NEAR STOCKBRIDGE, WI

GAGE HEIGHT, FEET

WATER YEAR OCTOBER 2013 TO SEPTEMBER 2014

DAILY MEAN VALUES

[e, estimated]

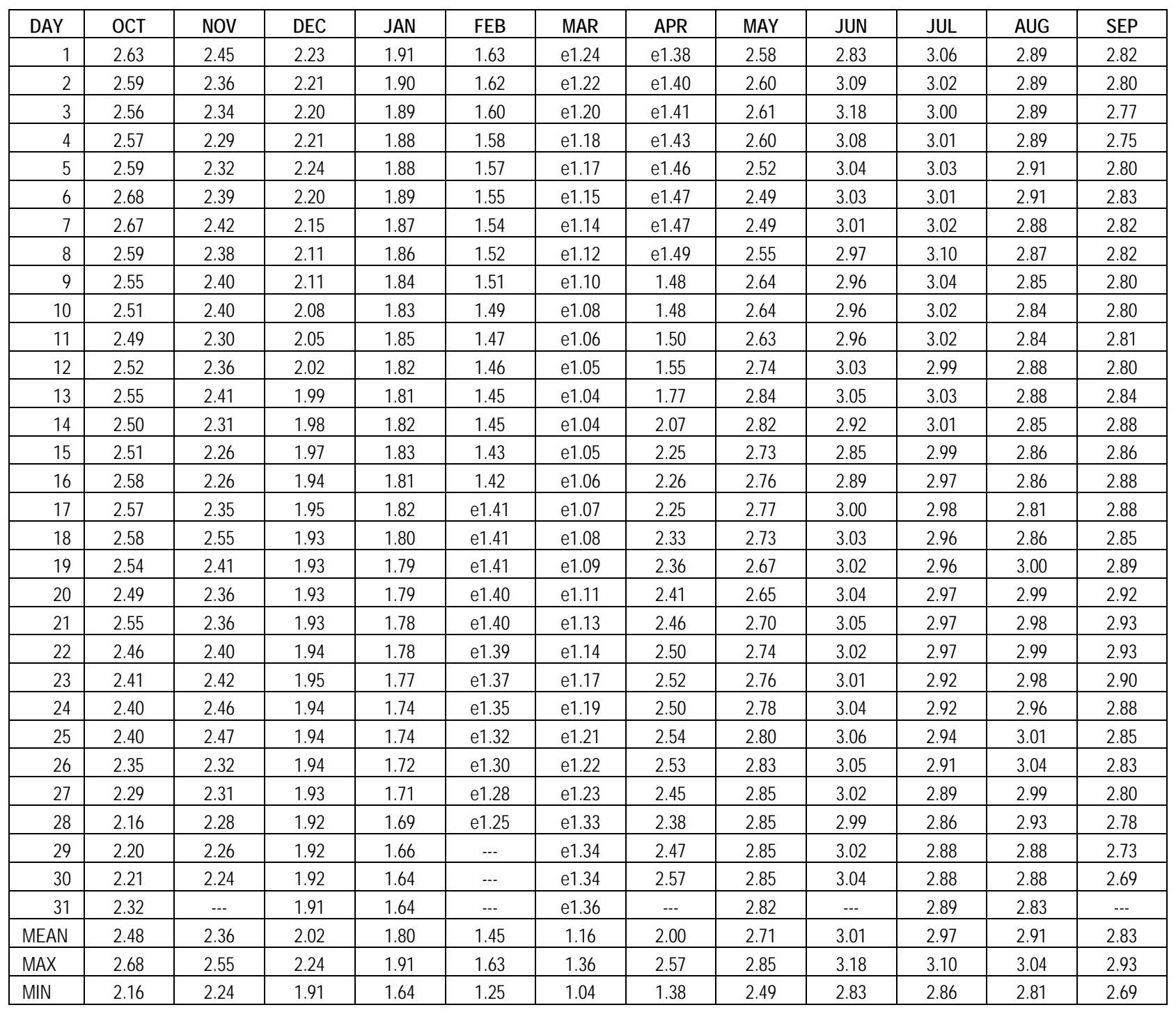




\section{WISCONSIN WATER SCIENCE CENTER PUBLICATIONS PERTAINING TO LAKES}

The reports published in a U.S. Geological Survey series are for sale by the U.S. Geological Survey, Box 25425, Federal Center, Denver, CO 80225. Prepayment is required. Remittance should be sent by check or money order payable to the U.S. Geological Survey. Prices can be obtained by writing to the above address or by calling (303) 236-7476. Reprints of journal articles may be obtained by writing directly to the author at U.S. Geological Survey, 8505 Research Way, Middleton, WI 53562.

2014

Brigham, M.E., Sandheinrich, M.B., Gay, D.A., Maki, R.P., Krabbenhoft, D.P., and Wiener, J.G., 2014, Lacustrine responses to decreasing wet mercury deposition rates-Results from a case study in northern Minnesota.: Environmental Science and Technology v. 48, no. 11, p. 6115-6123.

Read, J.S., Winslow, L.A., Hansen, G.J.A., Van Den Hoek, J., Hanson, P.C., Bruce, L.C., and Markfort, 2014, Simulating 2368 temperate lakes reveals weak coherence in stratification phenology: Ecological Modeling, v. 291, p. 142-150.

Rose, K.C., Winslow, L.A., Read, J.S., Read, E.K., Solomon, C.T., Adrian, R., and Hanson, P.C., 2014, Improving the precision of lake ecosystem metabolism estimates by identifying predictors of model uncertainty: Limnology and Oceanography Methods, v. 12, p. 303-312.

Winslow, L.A., Read, J.S., Hanson, P.C., and Stanley, E.H., 2014, Lake shoreline in the contiguous United States: quantity, distribution, and sensitivity to observation resolution: Freshwater Biology, v. 59, p. 213-223.

2013

Hunt, R.J., Walker, J.F., Selbig, W.R., Westenbroek, S.M., and Regan, R.S., 2013, Simulation of climatechange effects on streamflow, lake water budgets, and stream temperature using GSFLOW and SNTEMP, Trout Lake Watershed, Wisconsin: U.S. Geological Survey Scientific Investigations Report 2013-5159, 118 p., http://pubs.usgs.gov/sir/2013/5159/.

Juckem, P.J., and Robertson, D.M., 2013, Hydrology and water quality of Shell Lake, Washburn County, Wisconsin, with special emphasis on the effects of diversion and changes in water level on the water quality of a shallow terminal lake: U.S. Geological Survey Scientific Investigations Report 2013-5181, $77 \mathrm{p}$.

Meyer, M.W.; Walker, J.F.; Kenow, K.P.; Rasmussen, P.W.; Garrison, P.J.; Hanson, P.C.; Hunt, R.J., 2013. Potential effects of climate change on inland glacial lakes and implications for lake-dependent biota in Wisconsin: final report April 2013. Focus on Energy , x, 166 p.

Powers, S.M., Robertson, D.M., and Stanley, E.H., 2013, Effects of lakes and reservoirs on annual river nitrogen, phosphorus, and sediment export in agricultural and forested landscapes: Hydrological Processes, DOI: 10.1002/hyp.10083.

Watras, C.J., Morrow, M., Morrison, K., Scannell, S., Yaziciaglu, S., Read, J.S., Hu, Y.H., Hanson, P.C.,Kratz, T.K., 2013. Evaluation of wireless sensor networks (WSNs) for remote wetland monitoring: Design and initial results. Environmental Monitoring and Assessment. doi:10.1007/s10661-013-3424-8 
Juckem, P.F., Reneau, P.C., and Robertson, D.M., 2012, Estimation of natural historical flows for the Manitowish River near Manitowish Waters, Wisconsin: U.S. Geological Survey Scientific Invest. Report 2012-5135. $42 \mathrm{p}$.

Robertson, D.M., Garn, H.S., Rose, W.J., Juckem, P.J., and Reneau, P.C., 2012, Hydrology, water quality, and response to simulated changes in phosphorus loading of Mercer Lake, Iron County, Wisconsin, with emphasis on effects of wastewater discharges on water quality: U.S. Geological Survey Scientific Investigations Report 2012-5134, 58 p.

2011

Feinstein, D.T., Dunning, C.P., Juckem, P.F., and Hunt, R.J., 2010, Application of the Local Grid Refinement package to an inset model simulating the interactions of lakes, wells, and shallow groundwater, northwestern Waukesha County, Wisconsin: U.S. Geological Survey Scientific Investigations Report 2010-5214, $30 \mathrm{p}$.

Robertson, D.M. and Rose, W.J., 2011, Response in the trophic state of stratified lakes to changes in hydrology and water level: potential effects of climate change: Journal of Water and Climate Change, vol. 2. No. 1, p. 1-18.

2010

Garn, H.S., Robertson, D.M., Rose, W.J., and Saad, D.A., 2010, Hydrology, water quality, and response to simulated changes in phosphorus loading of Minocqua and Kawaguesaga Lakes, Oneida County, Wisconsin, with special emphasis on effects of urbanization: U.S. Geol. Survey Scientific Invest. Report 2010-5196.

Robertson, D.M., Rose, W.J., and Fitzpatrick, F.A., 2009, Water quality and hydrology of Silver Lake, Barron County, Wisconsin, with special emphasis on the responses of a terminal lake to changes in phosphorus loading and water level: U.S. Geol. Survey Scientific Invest. Report 2009-5077, 38 p.

Robertson, D.M., Rose, W.J., and Juckem, P.F., 2009, Water quality and hydrology of Whitefish Lake, Douglas County, Wisconsin, with special emphasis on the responses of an oligotrophic seepage lake to changes in phosphorus loading and water level: U.S. Geol. Survey Scientific Invest. Report 2009-5089, 41 p.

2008

Chung, E., Schladow, S.G., Perez-Losada, J., and Robertson, D.M., 2008, A linked hydrodynamic and water quality model for the Salton Sea: Hydrobiologia, v. 604, p. 57-75.

Robertson, D.M., and Rose, W.J., 2008, Water quality, hydrology, and simulated response to changes in phosphorus loading of Butternut Lake, Price and Ashland Counties, Wisconsin, with special emphasis on the effects of internal phosphorus loading in a polymictic lake: U.S. Geol. Survey Scientific Invest. Report 2008-5053, 46 p.

Robertson, D.M., and Schladow, S.G., 2008, Response in the water quality in the Salton Sea to changes in phosphorus loading: An empirical modeling approach: Hydrobiologia, v. 604, p. 5-19.

Robertson, D.M., Schladow, S.G., and Holdren, G.C., 2008, Long-term changes in the phosphorus loading to and trophic state of the Salton Sea: Hydrobiologia, v. 604, p. 21-36. 
Robertson, D.M., Garn, H.S., and Rose, W.J., 2007, Response of calcareous Nagawicka Lake, Wisconsin, to changes in phosphorus loading. Lake and Reservoir Management, Vol. 23, p. 298-312.

Walker, J.F., Saad, D.A., and Hunt, R.J., 2007, Dynamics of CFCs in northern temperate lakes and adjacent groundwater. Water Resources. Research 43(4) W04423, http://www.agu.org/pubs/crossref/2007/2005WR004647.shtml

2006

Garn, H.S., Robertson, D.M., Rose, W.J., Goddard, G.L., and Horwatich, J.A., 2006, Water quality, hydrology, and response to changes in phosphorus loading of Nagawicka Lake, a calcareous lake in Waukesha County, Wisconsin: U.S. Geological Survey Scientific Investigations Report: 2006-5273.

Hunt, R.J., Greb, S.R., Graczyk, D.J., 2006, Evaluating the effects of nearshore development on Wisconsin lakes. USGS Fact Sheet FS-2006-3033, 4 p.

Magnuson, J.J., Benson, B.J., Lenters, J.D, and Robertson, D.M., 2006, Climate driven variability and change, Chapter 7, In Magnuson, J.J., Kratz, T.K, and Benson, B.J eds. Long-term dynamics of lakes in the landscape, Oxford University Press, p 123-150.

Saad, D.A., 2005, Pesticides in surface water, bed sediment, and ground water adjacent to commercial cranberry bogs, Lac du Flambeau Reservation, Vilas County, Wisconsin: U.S. Geological Survey Scientific Investigations Report 2005-5262, 29 p.

\section{5}

Hunt, R.J., Feinstein, D.T., Pint, C.D., and Anderson, M.P., 2005, The importance of diverse data types to calibrate a watershed model of the Trout Lake Basin, northern Wisconsin: Journal of Hydrology doi:10.1016/j.jhydrol.2005.08.005.

Marzolf, G.R., and Robertson, D.M., 2005, Reservoir, In Encyclopedia of Hydrological Sciences: Anderson, M.G., and McDonnell, J.J., eds., v. 4, part 9, John Wiley \& Sons, p. 1675-1680.

Robertson, D.M., Rose, W.J., and Saad, D.A., 2005, Water quality, hydrology, and phosphorus loading to Little St. Germain Lake, Wisconsin, with special emphasis on the effects of winter aeration and groundwater inputs: U.S. Geological Survey Scientific Investigations Report 2005-5071, 36 p.

\section{4}

Dupre, D.H., and Robertson, D.M., 2004, Water quality of Nippersink Creek and Wonder Lake, McHenry County, Illinois, 1994-2001: U.S. Geological Survey Scientific Investigations Report 2004-5085.

Rose, W.J., Robertson, D.M., and Mergener, E.A., 2004, Water quality, hydrology, and the effects of changes in phosphorus loading to Pike Lake, Washington County, Wisconsin, with special emphasis on inlet-to-outlet short-circuiting: U.S. Geological Survey Scientific Investigations Report 2004-5141, 32 p.

2003

Dunning, C.P., Thomas, J.C., and Lin, Y.F., 2003, Simulation of the shallow aquifer in the vicinity of Silver Lake, Washington County, Wisconsin, using analytic elements: U.S. Geological Survey WaterResources Investigations Report 02-4204, 29 p. 
Fitzpatrick, F.A., Garrison, P.J., Fitzgerald, S.A., and Elder, J.F., 2003, Nutrient, trace-element, and ecological history of Musky Bay, Lac Courte Oreilles, Wisconsin, as inferred from sediment cores: U.S. Geological Survey Water-Resources Investigations Report 02-4225, 141 p.

Fitzpatrick, F.A., and Peppler, M.C., 2003, Sedimentation and sediment chemistry, Neopit Mill Pond, Menominee Indian Reservation, Wisconsin, 2001: U.S. Geological Survey Open-File Report 03-23, 58 p.

Garn, H.S., Elder, J.F., and Robertson, D.M., 2003, Why study lakes? An overview of U.S. Geological Survey lake studies in Wisconsin: U.S. Geological Survey Fact Sheet FS-063-03, 8 p.

Graczyk, D.G., Hunt, R.J., Greb, S.R., Buchwald, C.A., and Krohelski, J.T., 2003, Hydrology, water quality, and yields, from near-shore flows to four lakes in northern Wisconsin, 1999-2001: U.S. Geological Survey Water-Resources Investigations Report 03-4144. 64 p.

Hunt, R.J., 2003, Ground water-lake interaction modeling using the LAK3 Package for MODFLOW2000: Ground Water, vol. 41, no. 2, p. 114-118.

Hunt, R.J., Haitjema, H.M., Krohelski, J.T., and Feinstein, D.T., 2003, Simulating ground water-lake interactions: approaches and insights: Ground Water, vol. 41, no. 22, p. 227-237.

Hunt, R.J., Pint, C.D., and Anderson, M.P., 2003, Using diverse data types to calibrate a watershed model of the Trout Lake Basin, northern Wisconsin, In MODFLOW and More 2003-Understanding through modeling: Proceedings of the 5th International Conference of the International Ground Water Modeling Center. Golden, CO: Colorado School of Mines, p. 600-604.

Hunt, R.J., Saad, D.A., and Chapel, D.M., 2003, Numerical simulation of ground water flow in La Crosse County, Wisconsin and into nearby pools of the Mississippi River: U.S. Geological Survey WaterResources Investigations Report 03-4154. $36 \mathrm{p}$.

John, R., Pint, C.D., Anderson, M.P., and Hunt, R.J., 2003, The effects of potential climate change on lake levels and lake capture zones, In MODFLOW and More 2003-Understanding through modeling: Proceedings of the 5th International Conference of the International Ground Water Modeling Center. Golden, CO: Colorado School of Mines, p. 212-216.

Magnuson, J.J., Krohelski, J.T., Kunkel, K.E., and Robertson, D.M., 2003, Wisconsin's waters and climatehistorical changes and possible futures, In Wisconsin's waters: a confluence of perspectives: Transactions of the Wisconsin Academy, p. 23-36.

Pint, C.D., Hunt, R.J., and Anderson, M.P., 2003, Flow path delineation and ground water age, Allequash Basin, Wisconsin: Ground Water, vol. 41, no. 7, p. 895-902.

Robertson, D.M., Rose, W.J., and Garn, H.S., 2003, Water quality and the effects of changes in phosphorus loading, Red Cedar Lakes, Barron and Washburn Counties, Wisconsin: U.S. Geological Survey WaterResources Investigations Report 03-4238, 42 p.

Robertson, D.M., Rose, W.J., and Saad, D.A., 2003, Water quality and the effects of changes in phosphorus loading to Muskellunge Lake, Vilas County, Wisconsin: U.S. Geological Survey Water-Resources Investigations Report 03-4011, 18 p.

2002

Anderson, M.P., Hunt, R.J., Krohelski, J.T., and Chung, K., 2002, Using high hydraulic conductivity nodes to simulate seepage lakes: Ground Water 40(2), p. 119-124. 
Garn, H. S., 2002, Effects of lawn fertilizer on nutrient concentration in runoff from lakeshore lawns, Lauderdale Lakes, Wisconsin: USGS Water-Resources Investigations Report 02-4130, 6 p.

Kelson, V.A., Hunt, R.J., and Haitjema, H.M., 2002, Improving a regional model using reduced complexity and parameter estimation: Ground Water, vol. 40, no. 2, p. 138-149.

Krohelski, J.T., Lin, Y., Rose, W.J., and Hunt, R.J., 2002, Simulation of Fish, Mud and Crystal Lakes and the shallow ground-water system, Dane County, Wisconsin: USGS Water-Resources Investigations Report 02-20.

Krohelski, J.T., Rose, W.J., and Hunt, R.J., 2002, Hydrologic investigation of Powell Marsh and its relationship to Dead Pike Lake Vilas County, Wisconsin: USGS Water-Resources Investigations Report 02-4034.

Madenjian, C.P., Fahenstiel, G.L., Johengen, T.H., Nalepa, T.F., Venderploeg, H.A., Fleischer, G.W., Schneeberger, P.J., Benjamin, D.M., Smith, E.B., Bence, J.R., Rutherford, E.S., Lavis, D.S., Robertson, D.M., Jude, D.J., and Ebener, M.P., 2002, Dynamics of the Lake Michigan food web, 19702000: Canadian Journal of Fisheries and Aquatic Sciences, Vol. 59, p. 736-753.

Robertson, D.M., Goddard, G.L, Mergerer, E.A., Rose, W.J., and Garrison, P.J., 2002, Hydrology and water quality of Geneva Lake, Walworth County, Wisconsin: U.S. Geological Survey Water Resources Investigations Report 02-4039, 73 p.

Robertson, D.M., and Lenz, B.N., 2002, Response of the St. Croix River Pools, Wisconsin and Minnesota, to various phosphorus-loading scenarios: U.S. Geological Survey Water-Resources Investigations Report 02-4181, $36 \mathrm{p}$.

2001

Hunt, R.J., Bradbury, K.R., and Krohelski, J.T., 2001, The effects of large-scale pumping and diversion on the water resources in Dane County, Wisconsin: USGS Fact Sheet FS-127-01, 4 p.

Hunt, R.J., Haitjema, H.M., Krohelski, J.T., and Feinstein, D.T., 2001, Simulating groundwater-lake interactions with models: MODFLOW and analytic element approaches, In MODFLOW 2001 and Other Modeling Odysseys: Proceedings of the 4th International Conference of the International Ground Water Modeling Center. Golden, CO: Colorado School of Mines, p. 328-334.

Lin, Y., Krohelski, J.T., and Hunt, R.J., 2001, Simulation of lake stage in two seepage lakes in southcentral Wisconsin using the LAK3 package for MODFLOW, In MODFLOW 2001 and Other Modeling Odysseys: Proceedings of the 4th International Conference of the International Ground Water Modeling Center. Golden, CO: Colorado School of Mines, p. 411-417.

2000

Elder, J.F., Robertson, D.M., and Garrison, P.J., 2000, Chemical composition of surficial deposits in Geneva Lake, Wisconsin: U.S. Geological Survey Fact Sheet FS-121-00.

Elder J.F., Rybicki, N.B., Carter, V.P., and Weintraub, V., 2000, Sources and yields of dissolved carbon in northern Wisconsin stream catchments with differing amounts of peatland: Wetlands, vol. 20, no. 1 , p.113-125.

Hunt, R.J., Graczyk, D.J., and Rose, W.J., 2000, Water flows in the Necedah National Wildlife Refuge: U.S. Geological Survey Fact Sheet FS-068-00, 4 p. 
Hunt, R.J., Lin, J., Krohelski, J.T., and Juckem, P.F., 2000, Simulation of the shallow hydrologic system in the vicinity of Middle Genesee Lake, Wisconsin, using analytic elements and parameter estimation: U.S. Geological Survey Water Resources Investigations Report 00-4136, 16 p.

Lathrop, R.C., Carpenter, S.R., and Robertson, D.M., 2000, Interacting factors causing exceptional water clarity in Lakes Mendota and Monona, Wisconsin: Proc. of the International Limnological Society, SIL, Dublin, Ireland, August, 1998.

Magnuson, J.J., Robertson, D.M. Wynne, R.H., Benson, B.J., Livigstone, D.M., Arai, T., Assel, R.A., Barry, R.G., Card, V., Kuusisto, E., Granin, N.G., Prowse, T.D., Stewart, K.M., and Vuglinski, V.S., 2000, Historical trends in lake and river ice cover in the northern hemisphere: Science, Vol. 289, No. 5485, p. 1743-1746.

Magnuson, J.J., Wynne, R.H., Benson, B.J., and Robertson, D.M., 2000, Lake and river ice as a powerful indicator of past and present climates: Proc. of the International Limnological Society, SIL, Dublin, Ireland, August, 1998.

Robertson, D.M., 2000, One-dimensional simulation of stratification and dissolved oxygen in McCook Reservoir, Illinois: U.S. Geological Survey Water Resources Investigations Report 00-4258, 17 p.

Robertson, D.M., Goddard, G.L., Helsel, D.R., and MacKinnon, K.L., 2000, Rehabilitation of Delavan Lake, Wisconsin: Lake and Reservoir Management, vol. 20, vo. 3, p. 155-176.

Robertson, D.M., and Rose, W.J., 2000, Hydrology, water quality, and phosphorus loading of Little St. Germain Lake, Vilas County, Wisconsin: U.S. Geological Survey Water Resources Investigations Report 00-4209, 8 p.

Robertson, D.M., Wynne, R.H., and Chang, W.Y.B., 2000, Variability in ice cover across the northern hemisphere during the 1900's associated with El Nino events: Proceedings of the International Limnological Society, SIL, Dublin, Ireland, August, 1998.

Saad, D.A., and Robertson, D.M., 2000, Water-resources-related information for the St. Croix Reservation and vicinity, Wisconsin: U.S. Geological Survey Water Resources Investigations Report 00-4133, 65 p.

Johnson, G.P., Hornewer, N.J., Robertson, D.M., and Olson, D.T., 2000, Methodology, data collection, and data analysis for determination of water-mixing patterns induced by aerators and mixers: U.S. Geological Survey Water Resources Investigations Report 00-4101, 72 p.

Grannemann, N.G., Hunt, R.J., Nicholas, J.R. Reilly, T.E., and Winter, T.C., 2000, The importance of ground water to the Great Lakes Region: U.S. Geological Survey Water Resources Investigations Report 00-4008, 12 p.

1999

Lathrop, R.C., Carpenter, S.R., and Robertson, D.M., 1999, Summer water clarity responses to phosphorus, Daphnia grazing, and internal mixing in Lake Mendota: Limnology and Oceanography, vol. 44, no. 1, p. 137-146.

Krohelski, J.T., Feinstein, D.T., and Lenz, B.N., 1999, Simulation of stage and hydrologic budget for Shell Lake, Washburn County, Wisconsin: U.S. Geological Survey Water-Resources Investigations Report 99-4209, 23 p.

Panuska, J.C., and Robertson, D.M., 1999, Estimating phosphorus concentrations following alum treatment using apparent settling velocities: Lakes and Reservoir Management, vol. 15, no. 1, p. 28-38. 
Hunt, R.J, Anderson, M.P., and Kelson, V.A., 1998, Improving a complex finite-difference ground water flow model through the use of an analytic element screening model: Ground Water, vol. 36, no. 6, p. 10111017.

Hunt, R.J, Anderson, M.P., and Kelson, V.A., 1998, Linking an analytic element flow code to MODFLOWImplementation and benefits, In MODFLOW'98: Proceedings of the 3rd International Conference of the International Ground Water Center. Golden, CO: Colorado School of Mines, p 477-504.

Krabbenhoft, D.P., Gilmour, C.C., Benoit, J.M., Babiarz, C.L., Andren, A.W., and Hurley, J.P., 1998, Methyl mercury dynamics in littoral sediments of a temperate lake: Canadian Journal of Fisheries and Aquatic Sciences, vol. 55, p. 835-844.

Robertson, D.M., Elder, J.F., Goddard, G.L., and James, W.F., 1998, Dynamics in phosphorus retention in wetlands upstream of Delavan Lake, Wisconsin: Lakes and Reservoir Management, vol. 14, no. 4, p. 466-477.

Rose, W.J., and Robertson, D.M., 1998, Hydrology, water quality, and phosphorus loading of Kirby Lake, Barron County, Wisconsin: U.S. Geological Survey Fact Sheet FS-066-98, 4 p.

Walker, J.F. and Krabbenhoft, D.P., 1998, Groundwater and surface-water interactions in riparian and lakedominated systems, In Kendall, C. and McDonnell, J.J. eds., Isotope tracers in catchment hydrology, Elsevier Publishing, New York, 839 p.

1997

Elder, J.F., Manion, B.J., and Goddard, G.L., 1997, Mesocosm experiments to assess factors affecting phosphorus retention and release in an extended Wisconsin wetland: U.S. Geological Survey WaterResources Investigations Report 97-4272, 14 p.

Goddard, G.L., and Elder, J.F., 1997, Retention of sediments and nutrients in Jackson Creek Wetland near Delavan Lake, Wisconsin, 1993-95: U.S. Geological Survey Water-Resources Investigations Report 97-4014, $22 \mathrm{p}$.

Hornewer, N.J., Johnson, G.P., Robertson, D.M. and Hondzo, M., 1997, Field-scale tests for determining mixing patterns associated with coarse-bubble air diffuser configurations, Egan Quarry, Illinois, In Environmental and Coastal Hydraulics: Protecting the Aquatic Habitat, Proceedings of the International Association of Hydraulic Research, San Francisco, CA, USA, p. 57-63.

Robertson, D.M., 1997, Regionalized loads of sediment and phosphorus to Lakes Michigan and SuperiorHigh flow and long-term average: Journal of Great Lakes Research, vol. 23, p. 416-439.

1996

Anderson, W.L., Robertson, D.M., and Magnuson, J.J., 1996, Evidence of recent warming and El Ninorelated variation in ice breakup of Wisconsin lakes: Limnology and Oceanography, vol. 41, p. 815-821.

Elder, J.F. and Goddard, G.L., 1996, Sediment and nutrient trapping efficiency of a constructed wetland near Delavan Lake, Wisconsin, 1993-1995: U.S. Geological Survey Fact Sheet FS-232-96. 
Garn, H.S., Olson, D.L., Seidel, T.L., and Rose, W.J., 1996, Hydrology and water quality of Lauderdale Lakes, Walworth County, Wisconsin, 1993-94: U.S. Geological Survey Water-Resources Investigations Report 96-4235, $29 \mathrm{p}$.

Hunt, R.J. and Krohelski, J.T., 1996, The application of an analytical element model to investigate groundwater-lake interactions at Pretty Lake, Wisconsin: Lake and Reservoir Management, vol. 12, p. 487-495.

Imberger, J., Robertson, D.M., and Boland, K., 1996, Lake Number-A quantitative indicator of mixing to be used in water quality management: Scientific Impeller, Solna, Sweden, no. 4, p. 9-15.

Kammerer, P.A., Jr., 1996, Hydrology and water quality of Park Lake, South-central Wisconsin: U.S. Geological Survey Fact Sheet FS-197-96.

Robertson, D.M., Field, S.J, Elder, J.F., Goddard, G.L., and James, W.F., 1996, Phosphorus dynamics of Delavan Lake Inlet in southeastern Wisconsin: U.S. Geological Survey Water-Resources Investigations Report 96-4160, $18 \mathrm{p}$.

1995

Assel, R.A. and Robertson, D.M. 1995, Changes in winter air temperatures near Lake Michigan during 1851-1993, as determined from regional lake-ice records: Limnology and Oceanography, v. 40, p 165176.

Assel, R.A., Robertson, D.M., Hoff, M.H., and Selgeby, J.H., 1995, Climatic-change implications from longterm (1823-1994) ice records near the Laurentian Great Lakes: Annals of Glaciology, vol. 21, p. 383386.

Krabbenhoft, D.P., and Webster, K.E., 1995, Transient hydrogeological controls on the chemistry of a seepage lake: Water Resources Research, vol. 31, no. 9, p. 2295-2305.

Krohelski, J.T. and Batten, W.G., 1995, Simulation of stage and the hydrologic budget of Devils Lake, Sauk County, Wisconsin: U.S. Geological Survey Open-File Report 94-348, 22 p.

Wentz, D.A., Rose, W.J., and Webster, K.E., 1995, Long-term hydrologic and biogeochemical responses of a soft water seepage lake in north central Wisconsin: Water Resources Research, vol. 31, no. 1, p. 199-212.

1994

Elder, J.F., 1994, Distribution and grain-size partitioning of metals in bottom sediments of an experimentally acidified lake: Water Resources Bulletin, vol. 30, no. 2, p. 251-259.

Goddard, G.L., and Field, S.J., 1994, Hydrology and water quality of Whitewater and Rice Lakes in southeastern Wisconsin, 1990-91: U.S. Geological Survey Water-Resources Investigations Report 94$4101,36 \mathrm{p}$.

Greb, S.R., and Wentz, D.A., 1994, Chemical budgets, In Klepinger, K.E., ed., RILWAS 1983-86: Wisconsin Regional Integrated Lake Watershed Acidification Study, Volume 1: Madison, Wisconsin Department of Natural Resources, PUBL-RS-909-94, Chapter 7, 20 p. 
Hurley, J.P., Krabbenhoft, D.P., Babiarz, C.L., and Andren, A.W., 1994, Cycling processes of mercury across sediment/water interfaces in seepage lakes, In Baker, L.A. ed., Environmental Chemistry of Lakes and Reservoirs: Advances in Chemistry Series, American Chemical Society, Washington, D.C., p. 426-449.

Krabbenhoft, D.P., Bowser, C.J., Kendall, C., and Gat, J.R., 1994, Use of oxygen-19 and deuterium to assess the hydrology of ground-water/lake systems, In Baker, L.A. ed., Environmental Chemistry of Lakes and Reservoirs: Advances in Chemistry Series, American Chemical Society, Washington, D.C., p. 67-90.

Robertson, D.M., Anderson, W., and Magnuson, J.J., 1994, Relations between El Nino/Southern Oscillation events and the climate and ice cover of lakes in Wisconsin, In Greenland, D. ed., El Nino and LongTerm Ecological Research (LTER) Sites: Publication No. 18. LTER Network Office: University of Washington, Seattle, WA, USA., p. 48-57.

Robertson, D.M. and Imberger, J. 1994, Lake Number, a quantitative indicator of mixing used to estimate changes in dissolved oxygen, Internationale Revue der gesamten Hydrobiologie, v. 79, p. 159-176.

Watras, C.J., Bloom, N.S., Hurley, J.P., Fitzgerald, W.F., Andren, A.W., Krabbenhoft, D.P., and Porcella, D.B., 1994, Sources and fates of mercury and methylmercury in Wisconsin lakes, In Watras and Huckabee eds., Mercury as a Global Pollutant: Intergration and Synthesis, Lewis Pub., Chelsea, MI., p 153-177.

Wentz, D.A., 1994, Chemistry of snowpack and ground water, In Klepinger, K.E., ed., RILWAS 1983-86: Wisconsin regional integrated lake watershed acidification study, volume 1: Madison, Wisconsin Department of Natural Resources, PUBL-RS-909-94, chapter 6, 45 p.

Wentz, D.A., Krohelski, J.T., and Rose, W.J., 1994, Hydrology, In Klepinger, K.E., ed., RILWAS 1983-86: Wisconsin regional integrated lake watershed acidification study, volume 1: Madison, Wisconsin Department of Natural Resources, PUBL-RS-909-94, chapter 7, 74 p.

1993

Field, S.J., 1993, Hydrology and water quality of Powers Lake, southeastern Wisconsin: U.S. Geological Survey Water-Resources Investigations Report 90-4126, 36 p.

Field, S.J., 1993, Hydrology and water quality of Wind Lake in southeastern Wisconsin: U.S. Geological Survey Water-Resources Investigations Report 91-4107, $61 \mathrm{p}$.

House, L.B., 1993, Simulation of the effects of hypothetical residential development on water levels in Graber Pond, Middleton, Wisconsin: U.S. Geological Survey Water-Resources Investigations Report 92-4029, $10 \mathrm{p}$.

House, L.B., Waschbusch, R.J., and Hughes, P.E., 1993, Water quality of an urban wet detention pond in Madison, Wisconsin, 1987-88: U.S. Geological Survey Open-File Report 93-172, 57 p.

Hughes, P.E., 1993, Hydrology, water quality, trophic status, and aquatic plants of Fowler Lake, Wisconsin: U.S. Geological Survey Water-Resources Investigations Report 91-4076, 44 p.

Rose, W.J., 1993, Hydrology of Little Rock Lake in Vilas County, north-central Wisconsin: U.S. Geological Survey Water-Resources Investigations Report 93-4139, 22 p.

Rose, W.J., 1993, Water and phosphorus budgets and trophic state, Balsam Lake, northwestern Wisconsin, 1987-1989: U.S. Geological Survey Water-Resources Investigations Report 91-4125, 28 p. 
Elder, J.F., Krabbenhoft, D.P, and Walker, J.F., 1992, Water, energy, and biogeochemical budgets (WEBB) program: Data availability and research at the northern temperate lakes site, Wisconsin: U.S. Geological Survey Open-File Report 92-48, 15 p.

Krabbenhoft, D.P., and Babiarz, C.L., 1992, Role of groundwater transport in aquatic mercury cycling: Water Resources Research, vol. 28, no. 12, p. 3119-3128.

Krabbenhoft, D.P., and Krohelski, J.T., 1992, Data on water quality, lake sediment, and lake-level fluctuation, St. Croix Indian Reservation, Wisconsin, 1981-87: U.S. Geological Survey Open-File Report 92-26, $53 \mathrm{p}$.

Robertson, D.M., Ragotzkie, R.A., and Magnuson, J.J. 1992, Lake ice records used to detect historical and future climatic changes: Climatic Change, v. 21, p. 407-427.

1991

Wentz, D.A., and Rose, W.J., 1991, Hydrology of Lakes Clara and Vandercook in north-central Wisconsin: U.S. Geological Survey Water-Resources Investigations Report 89-4204, 24 p.

Watras, C.J., Andre, A.W., Bloom, N.S., Fitzgerald, W.F., Hurley, J.P., Krabbenhoft, D.P., Rada, R.G., Wiener, J.G., 1991, Mercury in temperate lakes: a mechanistic field study: Verhandlungen Internat. Verein. Limnologie, 24, p. 2199-2202.

Pre-1990

Walker, J.F., Pickard, S.A., and Sonzogni, W.C., 1989 Spreadsheet watershed modeling for nonpointsource pollution management in a Wisconsin basin: Water Resources Bulletin, vol. 25, no. 1, p. 139147.

Wentz, D.A., Garrison, P.J., and Bockheim, J.G., 1989, Section 7-Chemical input-output budgets, In Knauer, D., and Brouwer, S.A., eds., The Wisconsin regional integrated lake watershed acidification study (RILWAS)-1981-1983: Palo Alto, California, Electric Power Research Institute Report EA-6214, p. 7-1 to 7-30.

Wentz, D.A., and Rose, W.J., 1989, Interrelationships among hydrologic-budget components of a northern Wisconsin seepage lake and implications for acid-deposition modeling: Archives of Environmental Contamination and Toxicology, vol. 18, p. 147-155.

Wentz, D.A., Rose, W.J., and Krohelski, J.T., 1989, Section 5-Hydrologic component, in Knauer, D., and Brouwer, S.A., eds., The Wisconsin regional integrated lake watershed acidification study (RILWAS)— 1981-1983: Palo Alto, California, Electric Power Research Institute Report EA-6214, p. 5-1 to 5-77.

Field, S.J., and Duerk, M.D., 1988, Hydrology and water quality of Delavan Lake in southeastern Wisconsin: U.S. Geological Survey Water-Resources Investigations Report 87-4168, 61 p.

Krug, W.R., Ostenso, N.A., and Krohelski, J.T., 1988, Prediction of the effects of mine dewatering on four lakes near Crandon, Wisconsin, by use of a water-budget model: U.S. Geological Survey Open-File Report 87-471, 63 p. 
Wentz, D.A., Krohelski, J.T., Rose, W.J., Bockheim, J.G., Garrison, P.J., Knauer, D.R., and Goldstein, R.A., 1987, Hydrologic and chemical budgets of Wisconsin lakes, In Perry, R., Harrison, R.M., Bel, J.N.B., and Lester, J.N., eds., Acid Rain: Scientific and Technical Advances, Selper Ltd., London, p. 309-316.

House, L.B., 1986, Stage fluctuations of Wisconsin Lakes: Wisconsin Geological and Natural History Survey Information Circular No. 49, 84 p.

House, L.B., 1984, Effects of urbanization on three ponds in Middleton, Wisconsin: U.S. Geological Survey Water-Resources Investigations Report 84-4051, 17 p.

Krug, W.R., and House, L.B., 1984, Evaluation of alternative reservoir-management practices in the Rock River basin, Wisconsin: U.S. Geological Survey Water-Resources Investigations Report 83-4186, 21 p.

House, L.B., 1981, An assessment of streamflow, water quality, and the effects of construction on impoundment on Bridge Creek at Augusta, Wisconsin: U.S. Geological Survey Water-Resources Investigations Open-File Report 81-1192, 25 p.

Krug, W.R., 1981, Hydrologic effects of proposed changes in management practices, Winnebago Pool, Wisconsin: U.S. Geological Survey Water-Resources Investigations 80-107, 19 p.

Batten, W.G., and Hindall, S.M., 1980, Sediment deposition in the White River Reservoir, northwestern Wisconsin: U.S. Geological Survey Water-Supply Paper 2069, 30 p.

Novitzki, R.P., and Holmstrom, B.K., 1979, Monthly and annual water budgets of Lake Wingra, Madison, Wisconsin, 1971-77: U.S. Geological Survey Water-Resources Investigations 79-100, 31 p.

Rose, W.J., 1977, Hydrologic considerations associated with dredging spring ponds in Wisconsin: U.S. Geological Survey Water-Resources Investigations 77-18, 35 p.

Oakes, E.L., Hendrickson, G.E., and Zuehls, E.E., 1975, Hydrology of the Lake Wingra basin, Dane County, Wisconsin: U.S. Geological Survey Water-Resources Investigations 17-75, 31 p.

Novitzki, R.P., 1971, Hydrologic investigations of Heart Lake, Green Lake County, Wisconsin: U.S. Geological Survey Administrative Report, $9 \mathrm{p}$. 


\section{APPENDIX}

\section{Wisconsin Lakes Team \\ Quality-Assurance Plan}

Most lake studies and monitoring programs that are conducted by the USGS Wisconsin Water Science Center entail water sampling and analysis to determine water quality and biological productivity. Because all sampling and analysis is subject to error and random variablility, a certain proportion of the sampling effort should include quality-assurance samples. Sampling by the USGS was done by the Lake Studies Team of the USGS Wisconsin Water Science Center. This team implements a quality-assurance plan each year that involves collecting three types of samples from a subset of the lakes studied each year, which include blanks, replicates, and spikes (U.S. Geological Survey, Wisconsin Water Science Center Lake Studies Team). These samples are collected and/or prepared solely for the purpose of assessing the magnitude of error and random variability so that the accuracy and precision of all data can be evaluated. The plan for this quality-assurance sampling is described below.

Three types of QA/QC samples are collected:

\section{blanks}

Provide information about accuracy and errors due to treatment or reagents replicates provide information about precision (variability) standard additions (spikes)

provide information about accuracy and matrix interferences

\section{Blank Sampling}

B1. A preservation blank consists of deionized water or inorganic blank water, to which is added any reagents or preservatives that are normally added to natural water samples. The blank is not taken to the field, but is shipped to the laboratory for analysis along with the natural water samples.

This blank sample is analyzed for the Nutrient Group ${ }^{1}$ and chlorophyll-a.

B2. A field blank consists of deionized water or inorganic blank water treated exactly the same as regular samples. During winter, the field blank is analyzed for total phosphorus (TP) only; during summer, it is analyzed for TP and chlorophyll-a, and in the spring it is analyzed for the Nutrient Group and chlorophyll-a.

${ }^{1}$ Nutrient Group = all phosphorus and nitrogen species that are commonly determined in lakes (total phosphorus, nitrate + nitrite, ammonia, total Kjeldahl nitrogen, total nitrogen) 


\section{Replicate Sampling}

Triplicate samples are taken near water surface in summer for analysis of total phosphorus and chlorophyll-a. For a portion of the sites where surface triplicates are collected, a set of triplicate samples is also taken from near-bottom water, for analysis of total phosphorus.

Triplicate samples collected in the spring are taken near the water surface for analysis of the Nutrient Group.

\section{Standard Addition Testing}

Replicate samples are collected for a standard addition (spike) test, which consists of an addition of a prepared phosphorus solution (standard) of known volume and concentration, such that the expected result of analysis is the natural water TP concentration plus the known addition. One sample from each set will receive no spike (the mean of these gives the natural water TP concentration).

Data and results of replicate sampling and field blank testing for the past five years are shown in Table A1. 
Table A1. Analyses of replicate samples from Wisconsin lakes in water years 2009-2014. See text for procedures used. Phosphorus data in milligrams per liter; chlorophyll data in micrograms per liter. Symbol "<" indicates less than given detection limit (DL); mean and standard deviation not calculated for datasets containing values less than DL.

\begin{tabular}{|c|c|c|c|c|c|c|c|c|}
\hline Parameter & Lake & Date & & Replica & e Data & Mean & $\begin{array}{l}\text { Standard } \\
\text { Deviation }\end{array}$ & $\begin{array}{l}\text { Percent } \\
\text { Standard } \\
\text { Deviation }\end{array}$ \\
\hline \multirow{17}{*}{ Total Phosphorus } & Beulah & 2/23/09 & 0.013 & 0.013 & & 0.013 & 0.000 & 0.0 \\
\hline & Beulah & $8 / 24 / 09$ & 0.017 & 0.017 & & 0.017 & 0.000 & 0.0 \\
\hline & Delavan & 9/15/09 & 0.035 & 0.031 & 0.031 & 0.032 & 0.002 & 7.1 \\
\hline & Beulah & 8/19/10 & 0.015 & 0.016 & & 0.016 & 0.001 & 4.6 \\
\hline & Big Cedar, South & $8 / 24 / 10$ & 0.015 & 0.014 & & 0.015 & 0.001 & 4.9 \\
\hline & Powers & $8 / 30 / 10$ & 0.021 & 0.022 & & 0.022 & 0.001 & 3.3 \\
\hline & Beulah & 8/10/11 & 0.140 & 0.170 & & 0.155 & 0.021 & 13.7 \\
\hline & Oconomowoc & $7 / 25 / 11$ & 0.013 & 0.010 & 0.012 & 0.012 & 0.002 & 13.1 \\
\hline & Powers & $7 / 25 / 11$ & 0.017 & 0.018 & 0.020 & 0.018 & 0.002 & 8.3 \\
\hline & Wind & $7 / 25 / 11$ & 0.018 & 0.019 & 0.021 & 0.019 & 0.002 & 7.9 \\
\hline & Anvil & $7 / 17 / 12$ & 0.024 & 0.035 & 0.028 & 0.029 & 0.006 & 19.2 \\
\hline & Beulah & $8 / 29 / 12$ & 0.014 & 0.015 & 0.016 & 0.015 & 0.001 & 6.7 \\
\hline & Big Cedar, South & $8 / 24 / 12$ & 0.011 & 0.011 & 0.011 & 0.011 & 0.000 & 0.0 \\
\hline & Little Cedar, S. & $8 / 24 / 12$ & 0.012 & 0.013 & & 0.013 & 0.001 & 5.7 \\
\hline & Powers & $8 / 30 / 12$ & 0.015 & 0.015 & 0.016 & 0.015 & 0.001 & 3.8 \\
\hline & Anvil & $10 / 16 / 12$ & 0.180 & 0.180 & & 0.180 & 0.000 & 0.0 \\
\hline & Beulah & $8 / 14 / 13$ & 0.170 & 0.170 & 0.170 & 0.170 & 0.000 & 0.0 \\
\hline \multirow{5}{*}{$\begin{array}{l}\text { Total Phosphorus, near } \\
\text { bottom }\end{array}$} & Big Cedar, South & $8 / 24 / 10$ & 0.081 & 0.067 & & 0.074 & 0.010 & 13.4 \\
\hline & Powers & $8 / 30 / 10$ & 0.036 & 0.038 & & 0.037 & 0.001 & 3.8 \\
\hline & Anvil & $7 / 17 / 12$ & 0.028 & 0.025 & 0.031 & 0.028 & 0.003 & 10.7 \\
\hline & Big Cedar, South & $8 / 24 / 12$ & 0.025 & 0.025 & 0.028 & 0.026 & 0.002 & 6.7 \\
\hline & Little Cedar, S. & $8 / 24 / 12$ & 0.168 & 0.170 & & 0.169 & 0.001 & 0.8 \\
\hline \multirow{7}{*}{ Dissolved Phosphorus } & Beulah & $2 / 23 / 09$ & $<0.002$ & $<0.002$ & & NA & NA & NA \\
\hline & Beulah & $8 / 24 / 09$ & $<0.002$ & $<0.002$ & & NA & NA & NA \\
\hline & Beulah & $8 / 19 / 10$ & $<0.002$ & $<0.002$ & & NA & NA & NA \\
\hline & Beulah & $8 / 10 / 11$ & $<0.002$ & $<0.002$ & & NA & NA & NA \\
\hline & Anvil & $7 / 17 / 12$ & 0.007 & $<0.002$ & 0.003 & NA & NA & NA \\
\hline & Beulah & $8 / 29 / 12$ & $<0.002$ & $<0.002$ & $<0.002$ & NA & NA & NA \\
\hline & Beulah & $8 / 14 / 13$ & $<0.002$ & $<0.002$ & $<0.002$ & NA & NA & NA \\
\hline \multirow{7}{*}{ Dissolved Ammonia } & Beulah & 2/23/09 & 0.211 & 0.204 & & 0.208 & 0.005 & 2.4 \\
\hline & Beulah & $8 / 24 / 09$ & 0.032 & 0.035 & & 0.034 & 0.002 & 6.3 \\
\hline & Beulah & 8/19/10 & $<0.015$ & 0.030 & & NA & NA & NA \\
\hline & Beulah & $8 / 10 / 11$ & 0.032 & 0.029 & & 0.031 & 0.002 & 7.0 \\
\hline & Anvil & $7 / 17 / 12$ & $<0.015$ & 0.019 & 0.018 & NA & NA & NA \\
\hline & Beulah & $8 / 29 / 12$ & $<0.015$ & $<0.015$ & $<0.015$ & NA & NA & NA \\
\hline & Beulah & $8 / 14 / 13$ & $<0.015$ & $<0.015$ & $<0.015$ & NA & NA & NA \\
\hline \multirow{7}{*}{ Total Kjeldahl Nitrogen } & Beulah & $2 / 23 / 09$ & 0.660 & 0.690 & & 0.675 & 0.021 & 3.1 \\
\hline & Beulah & $8 / 24 / 09$ & 0.160 & 0.530 & & 0.345 & 0.262 & 75.8 \\
\hline & Beulah & $8 / 19 / 10$ & 0.580 & 0.680 & & 0.63 & 0.071 & 11.2 \\
\hline & Beulah & $8 / 10 / 11$ & 0.560 & 0.460 & & 0.510 & 0.071 & 13.9 \\
\hline & Anvil & $7 / 17 / 12$ & 0.280 & 0.390 & 0.410 & 0.360 & 0.070 & 19.4 \\
\hline & Beulah & $8 / 29 / 12$ & 0.800 & 0.500 & 0.460 & 0.587 & 0.186 & 31.7 \\
\hline & Beulah & $8 / 14 / 13$ & 0.420 & 0.640 & & 0.530 & 0.156 & 29.4 \\
\hline
\end{tabular}




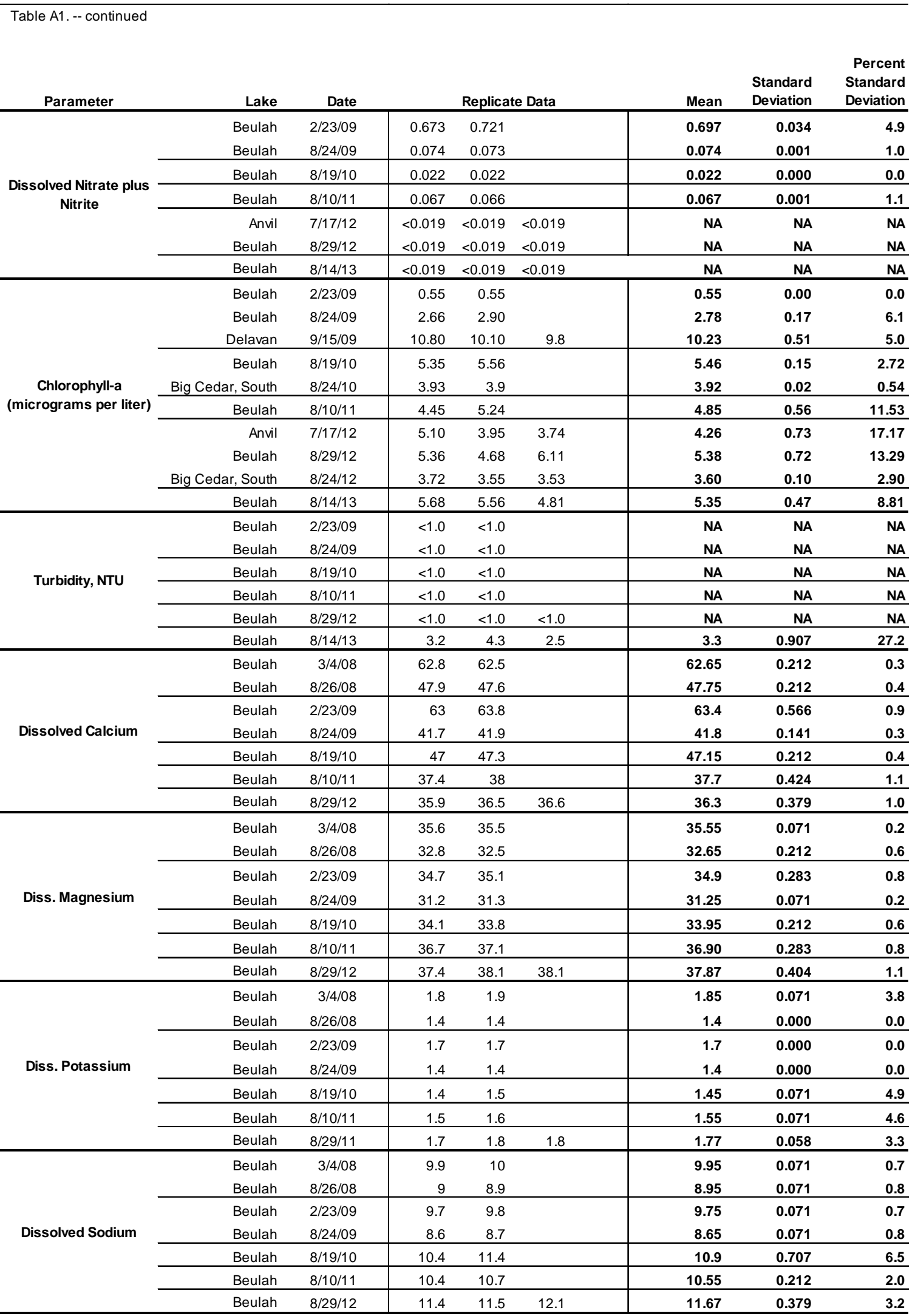




\begin{tabular}{|c|c|c|c|c|c|c|c|c|}
\hline Table A1. -- continued & Lake & Date & & Replica & Data & Mean & $\begin{array}{l}\text { Standard } \\
\text { Deviation }\end{array}$ & $\begin{array}{r}\text { Percent } \\
\text { Standard } \\
\text { Deviation }\end{array}$ \\
\hline \multirow{6}{*}{ ANC as $\mathrm{CaCO} 3$} & Beulah & $2 / 23 / 09$ & 258 & 256 & & 257 & 1.414 & 0.6 \\
\hline & Beulah & $8 / 24 / 09$ & 209 & 209 & & 209 & 0.000 & 0.0 \\
\hline & Beulah & $8 / 19 / 10$ & 208 & 207 & & 207.5 & 0.707 & 0.3 \\
\hline & Beulah & $8 / 10 / 11$ & 194 & 194 & & 194 & 0.000 & 0.0 \\
\hline & Beulah & $8 / 29 / 12$ & 206 & 205 & 205 & 205.3 & 0.577 & 0.3 \\
\hline & Beulah & $8 / 14 / 13$ & 221 & 222 & 221 & 221.3 & 0.577 & 0.3 \\
\hline \multirow{7}{*}{ Diss. Chloride } & Beulah & $3 / 4 / 08$ & 23.5 & 23.7 & & 23.6 & 0.141 & 0.6 \\
\hline & Beulah & $8 / 26 / 08$ & 21 & 20.9 & & 20.95 & 0.071 & 0.3 \\
\hline & Beulah & 2/23/09 & 23 & 22.9 & & 22.95 & 0.071 & 0.3 \\
\hline & Beulah & $8 / 24 / 09$ & 21.6 & 21.4 & & 21.5 & 0.141 & 0.7 \\
\hline & Beulah & $8 / 19 / 10$ & 21.6 & 21.5 & & 21.55 & 0.071 & 0.3 \\
\hline & Beulah & $8 / 10 / 11$ & 24.7 & 24.5 & & 24.6 & 0.141 & 0.6 \\
\hline & Beulah & $8 / 29 / 12$ & 26.9 & 27.1 & 27.1 & 27.03 & 0.115 & 0.4 \\
\hline \multirow{6}{*}{ Dissolved Silica } & Beulah & $2 / 23 / 09$ & 14.8 & 15 & & 14.9 & 0.141 & 0.9 \\
\hline & Beulah & $8 / 24 / 09$ & 11.3 & 11.3 & & 11.3 & 0.000 & 0.0 \\
\hline & Beulah & $8 / 19 / 10$ & 18.1 & 18.1 & & 18.1 & 0.000 & 0.0 \\
\hline & Beulah & $8 / 10 / 11$ & 12.7 & 12.7 & & 12.7 & 0.00 & 0.0 \\
\hline & Beulah & $8 / 29 / 12$ & 14.6 & 14.5 & 14.5 & 14.5 & 0.06 & 0.4 \\
\hline & Beulah & $8 / 14 / 13$ & 8.92 & 9.1 & 8.87 & 9.0 & 0.12 & 1.3 \\
\hline \multirow{6}{*}{ Dissolved Sulfate } & Beulah & $2 / 23 / 09$ & 30.5 & 30.8 & & 30.65 & 0.212 & 0.7 \\
\hline & Beulah & $8 / 24 / 09$ & 27.7 & 27.8 & & 27.75 & 0.071 & 0.3 \\
\hline & Beulah & $8 / 19 / 10$ & 25.9 & 25.8 & & 25.85 & 0.071 & 0.3 \\
\hline & Beulah & $8 / 10 / 11$ & 29.3 & 29.2 & & 29.25 & 0.071 & 0.2 \\
\hline & Beulah & $8 / 29 / 12$ & 32.5 & 32.8 & 32.5 & 32.6 & 0.173 & 0.5 \\
\hline & Beulah & $8 / 14 / 13$ & 25 & 25.3 & 25.3 & 25.2 & 0.173 & 0.7 \\
\hline \multirow{7}{*}{ Dissolved Iron } & Beulah & $3 / 4 / 08$ & $<100$ & $<100$ & & NA & NA & NA \\
\hline & Beulah & $8 / 26 / 08$ & $<100$ & $<100$ & & NA & NA & NA \\
\hline & Beulah & $2 / 23 / 09$ & $<100$ & $<100$ & & NA & NA & NA \\
\hline & Beulah & $8 / 24 / 09$ & $<100$ & $<100$ & & NA & NA & NA \\
\hline & Beulah & $8 / 19 / 10$ & $<100$ & $<100$ & & NA & NA & NA \\
\hline & Beulah & $8 / 10 / 11$ & $<100$ & $<100$ & & NA & NA & NA \\
\hline & Beulah & $8 / 29 / 12$ & $<100$ & $<100$ & $<100$ & NA & NA & NA \\
\hline \multirow{5}{*}{ Diss. Manganese } & Beulah & $8 / 26 / 08$ & $<0.5$ & $<0.5$ & & NA & NA & NA \\
\hline & Beulah & $2 / 23 / 09$ & $<1.0$ & $<1.0$ & & NA & NA & NA \\
\hline & Beulah & $8 / 24 / 09$ & $<1.0$ & $<1.0$ & & NA & NA & NA \\
\hline & Beulah & $8 / 10 / 11$ & $<1.0$ & $<1.0$ & & NA & NA & NA \\
\hline & Beulah & $8 / 29 / 12$ & $<1.0$ & $<1.0$ & $<1.0$ & NA & NA & NA \\
\hline \multirow{6}{*}{ Dissolved Solids } & Beulah & $2 / 23 / 09$ & 350 & 346 & & 348 & 2.83 & 0.8 \\
\hline & Beulah & $8 / 24 / 09$ & 312 & 312 & & 312 & 0.00 & 0.0 \\
\hline & Beulah & $8 / 19 / 10$ & 284 & 286 & & 285 & 1.41 & 0.5 \\
\hline & Beulah & $8 / 10 / 11$ & 270 & 272 & & 271 & 1.41 & 0.5 \\
\hline & Beulah & $8 / 29 / 12$ & 282 & 276 & 278 & 279 & 3.06 & 1.1 \\
\hline & Beulah & $8 / 14 / 13$ & 312 & 298 & 318 & 309 & 10.3 & 3.3 \\
\hline
\end{tabular}


Table A2. Data from tests of blanks, 2009-2014. All data in milligrams per liter, unless otherwise indicated. $<=$ less than given detection limit; $\mathrm{E}=$ estimated value .

Delavan Lake. Analyses at USGS National Water Quality Laboratory, Lakewood, CO.

\begin{tabular}{lc} 
Parameter & $9 / 14 / 09$ \\
\hline Total Phosphorus & $<0.008$ \\
Dissolved orthophosphate & $<0.008$ \\
Chlorophyll a & --- \\
\hline
\end{tabular}

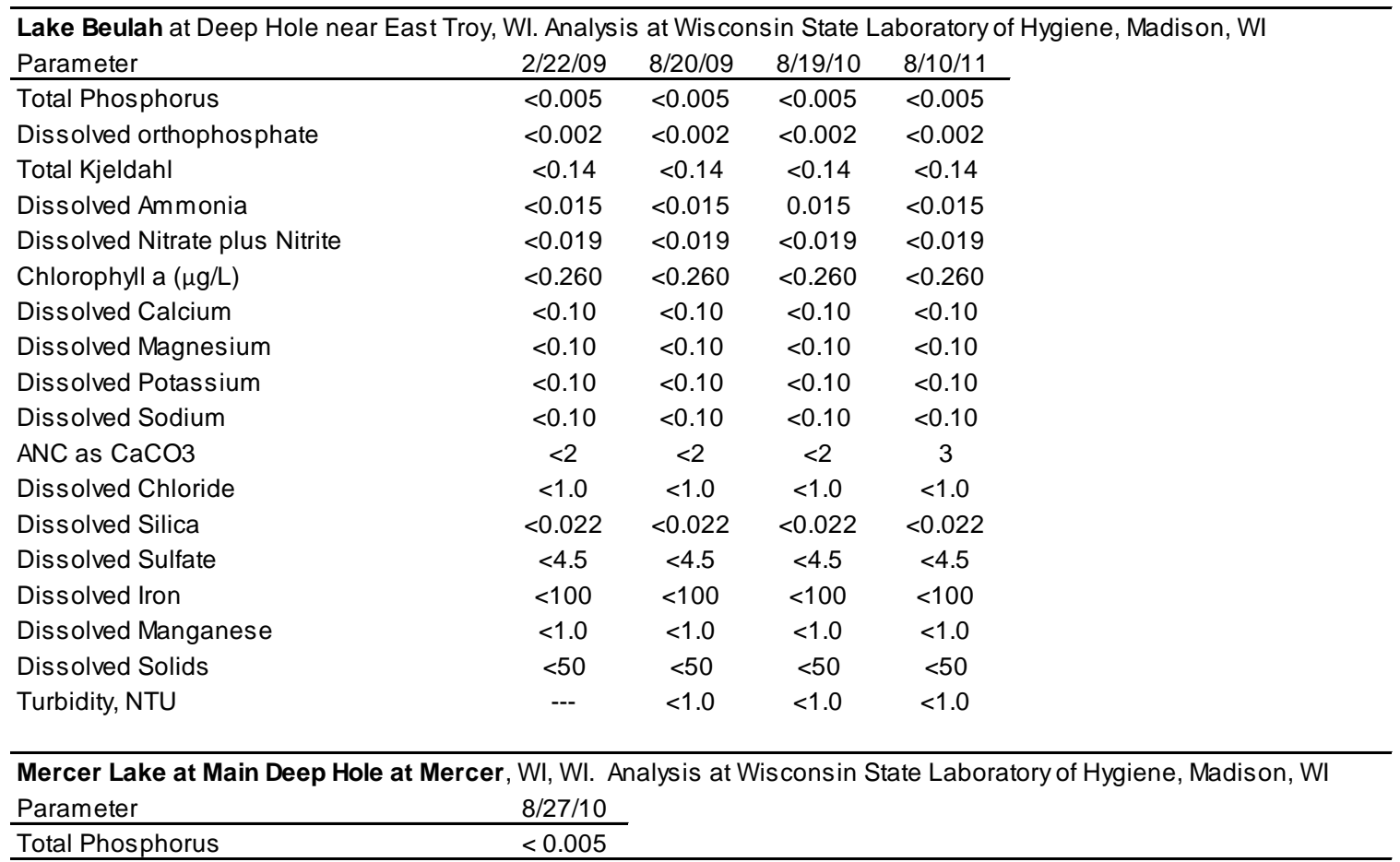

Wind Lake at Wind Lake, WI. Analyses at Wisconsin State Laboratory of Hygiene, Madison, WI

Parameter 8/30/10

Total Phosphorus $<0.005$

Chlorophyll a (ug/L)

\begin{tabular}{lc}
\hline Silver Lake near West Bend, WI. Analyses at Wisconsin State Laboratory of Hygiene, Madison, WI \\
Parameter & $8 / 31 / 09$ \\
\hline Total Phosphorus & $<0.005$ \\
Chlorophyll a (ug/L) & $<0.260$ \\
\hline
\end{tabular}



